\title{
Ammonia and glutamine metabolism during liver failure : studies in the rat
}

Citation for published version (APA):

Dejong, C. H. C. (1993). Ammonia and glutamine metabolism during liver failure : studies in the rat. [Doctoral Thesis, Maastricht University]. Datawyse / Universitaire Pers Maastricht. https://doi.org/10.26481/dis.19930115cd

Document status and date:

Published: 01/01/1993

DOI:

$10.26481 /$ dis. $19930115 \mathrm{~cd}$

Document Version:

Publisher's PDF, also known as Version of record

\section{Please check the document version of this publication:}

- A submitted manuscript is the version of the article upon submission and before peer-review. There can be important differences between the submitted version and the official published version of record.

People interested in the research are advised to contact the author for the final version of the publication, or visit the DOI to the publisher's website.

- The final author version and the galley proof are versions of the publication after peer review.

- The final published version features the final layout of the paper including the volume, issue and page numbers.

Link to publication

\footnotetext{
General rights rights.

- You may freely distribute the URL identifying the publication in the public portal. please follow below link for the End User Agreement:

www.umlib.nl/taverne-license

Take down policy

If you believe that this document breaches copyright please contact us at:

repository@maastrichtuniversity.nl

providing details and we will investigate your claim.
}

Copyright and moral rights for the publications made accessible in the public portal are retained by the authors and/or other copyright owners and it is a condition of accessing publications that users recognise and abide by the legal requirements associated with these

- Users may download and print one copy of any publication from the public portal for the purpose of private study or research.

- You may not further distribute the material or use it for any profit-making activity or commercial gain

If the publication is distributed under the terms of Article $25 \mathrm{fa}$ of the Dutch Copyright Act, indicated by the "Taverne" license above, 


\section{AMMONIA AND GLUTAMINE METABOLISM}

DURING LIVER FAILURE

Studies in the rat 


\title{
AMMONIA AND GLUTAMINE METABOLISM DURING LIVER FAILURE
}

\author{
Studies in the rat
}

\section{PROEFSCHRIFT}

ter verkrijging van de graad van doctor

aan de Rijksuniversiteit Limburg te Maastricht, op gezag van de Rector Magnificus, Prof.Mr. M.J. Cohen, volgens het besluit van het College van Dekanen, in het openbaar te verdedigen op vrijdag, 15 januari 1993 om 16.00 uur

door

Cornelis Hubertus Carolus Dejong

geboren 14 januari 1962 te Maastricht

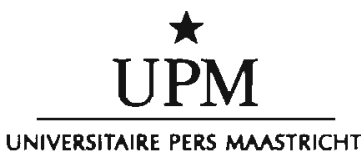




\section{Promotor}

Prof.dr.P.B. Soeters

\section{Co-promotor}

Dr. N.E.P. Deutz

\section{Beoordelingscommissie}

Prof.dr. H.C. Hemker (voorzitter)

Prof.dr. P.W. de Leeuw

Dr. A.J. Meijer (afdeling Biochemie, Universiteit van Amsterdam)

Prof.dr.R.S. Reneman

Prof.dr. M.J. Rennie (afdeling Fysiologie, Universiteit van Dundee, Engeland)

\section{CIP-DATA KONINKLIJKE BIBLIOTHEEK, DEN HAAG}

Dejong, Comelis Hubertus Carolus

Ammonia and glutamine metabolism during liver failure :

studies in the rat / Comelis Hubertus Carolus Dejong. -

Maastricht : Universitaire Pers Maastricht. - IIl.

Thesis Maastricht. - With ref. - With summary in Dutch.

ISBN 90-5278-056-0

NUGI 743

Subject headings: liver failure / ammonia/glutamine.

Layout: C.H.C. Dejong

Boekproduktie: Universitaire Pers Maastricht, T.R. Leliveld, Beeldsnijdersdreef 85, 6216 EA Maastricht

De druk van dit proefschrift werd financieel mogelijk gemaakt door Nutricia Nederland BV, de Stichting Dr. Ir. J.H.J. van de Laar, Zyma Nederland BV, Glaxo BV en Duphar Nederland BV. 
In memory of my mother, Anne

To Odile, Giulia and Anne-Linde

To my father, Jean 


\section{CONTENTS}

$\begin{array}{lll}\text { Chapter } 1 \quad \text { Introduction } & 9\end{array}$

Chapter $2 \quad$ Models \& methods

Chapter 3 Effects of starvation on interorgan amino acid and ammonia exchange

Chapter 4 Effects of enterectomy on interorgan amino acid and ammonia exchange

Chapter 5 Gut and muscle during acute liver failure

Chapter 6 Cerebral cortex during acute liver failure

a. Blood flow measurement

b. Ammonia and amino acid exchange

Chapter $7 \quad$ Kidney during acute liver failure

Chapter $8 \quad$ Muscle during chronic liver failure

Chapter 9 Gut during chronic liver failure

Chapter 10 Cerebral cortex during chronic liver failure

Chapter 11 Kidney during chronic liver failure

Chapter 12 Summary and conclusions

Dankwoord

Curriculum vitae

Publications 


\section{CONTENTS}

$\begin{array}{llr}\text { Chapter } 1 \text { Introduction } & 9\end{array}$

Chapter $2 \quad$ Models \& methods 31

Chapter 3 Effects of starvation on interorgan amino acid and ammonia exchange 41

Chapter 4 Effects of enterectomy on interorgan amino acid and ammonia exchange 51

Chapter $5 \quad$ Gut and muscle during acute liver failure 65

$\begin{array}{lll}\text { Chapter } 6 & \text { Cerebral cortex during acute liver failure } & 83\end{array}$

a. Blood flow measurement

b. Ammonia and amino acid exchange

$\begin{array}{lll}\text { Chapter } 7 & \text { Kidney during acute liver failure } & 107\end{array}$

$\begin{array}{lll}\text { Chapter } 8 & \text { Muscle during chronic liver failure } & 123\end{array}$

$\begin{array}{lll}\text { Chapter } 9 & \text { Gut during chronic liver failure } & 137\end{array}$

Chapter $10 \quad$ Cerebral cortex during chronic liver failure 151

$\begin{array}{lll}\text { Chapter } 11 & \text { Kidney during chronic liver failure } & 167\end{array}$

$\begin{array}{lll}\text { Chapter } 12 & \text { Summary and conclusions } & 185\end{array}$

$\begin{array}{ll}\text { Dankwoord } & 197\end{array}$

$\begin{array}{ll}\text { Curriculum vitae } & 199\end{array}$

$\begin{array}{ll}\text { Publications } & 200\end{array}$ 


\section{ABBREVIATIONS}

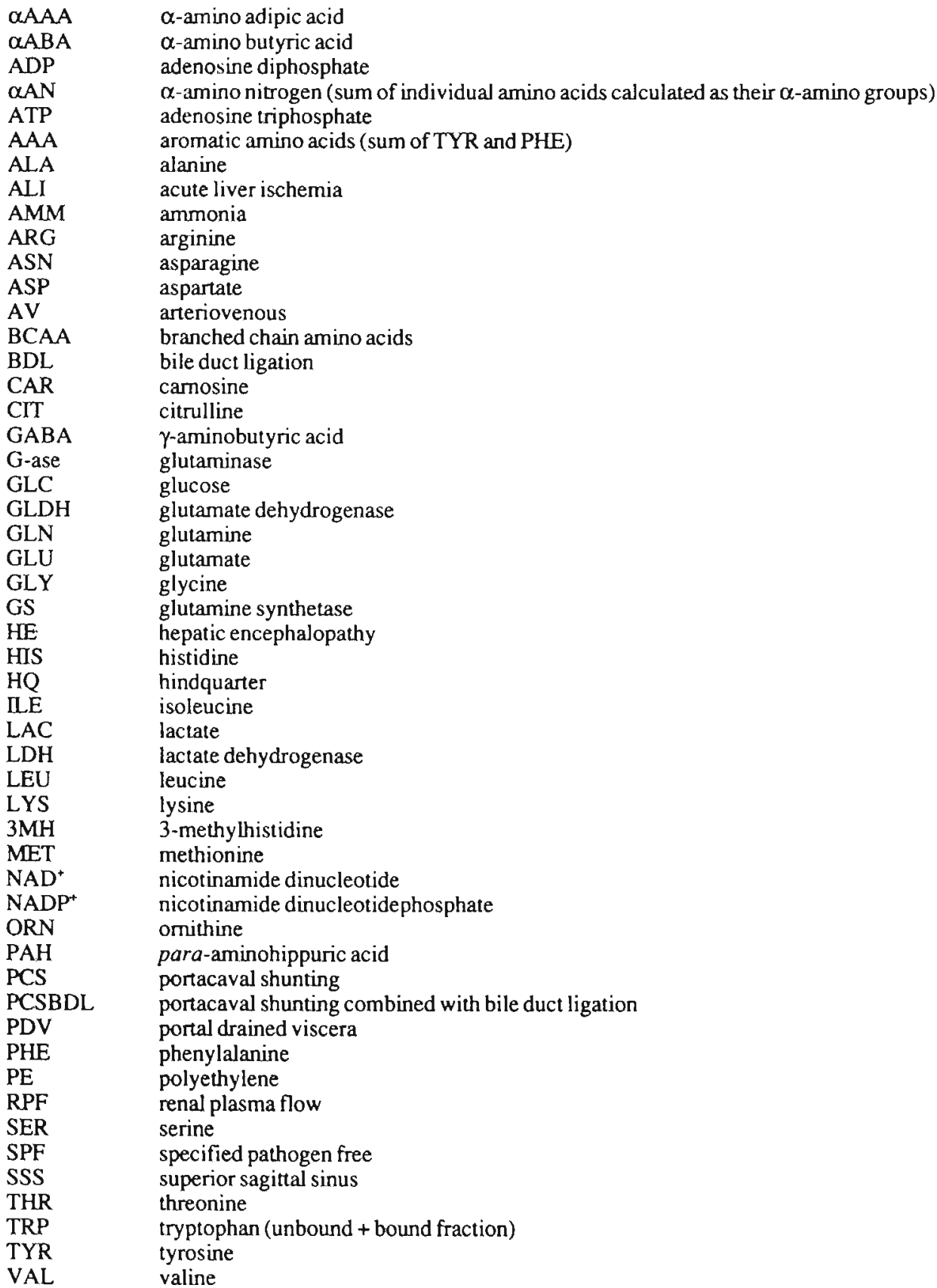




\section{Chapter 1}

\section{Introduction}

Partly published as: N.E.P. Deutz, C.H.C. Dejong, P.B. Soeters. Ammonia and glutamine metabolism during liver insufficiency: the muscle-gut-liver axis. It $J$ Gastroenterol 1993 ; in press.

\section{GENERAL INTRODUCTION}

Liver disease constitutes an important cause of morbidity and mortality. In surgical practice, the presence of liver disease adversely affects the outcome of any surgical procedure. Liver disease is more commonly present in patients undergoing surgery than is usually recognized. For example, patients receiving parenteral nutrition during prolonged periods of time often present symptoms and signs of cholestatic liver disease. Similarly, liver dysfunction is often temporarily present in patients undergoing partial hepatectomy for malignant disease, as well as in patients with bile obstruction due to gall stones or malignant disease. Also, it is well known that septic surgical patients often develop jaundice as a result of hemolysis and deficient hepatic function.

Because the liver has a key function in nitrogen metabolism, energy homeostasis and several detoxification processes, liver disease and the resulting hepatocellular failure adversely affect these processes and induce profound disturbances in nitrogen homeostasis. A crucial feature in this context is the diminished hepatic urea synthesis capacity, leading to an impaired capacity to detoxify ammonia. This diminished ammonia detoxification capacity combined with the usually existing intra- and/or extrahepatic shunting contributes to the development of systemic hyperammonemia $(1,2)$. Because glutamine synthesis has been suggested to be the most important alternative pathway for ammonia detoxification during diminished urea synthesis, systemic hyperammonemia in turn has a considerable impact on the metabolism of the non-essential amino acid glutamine. These disturbances in nitrogen homeostasis have been and still are subject to extensive research (see references attached to this chapter). Specifically, the relation between ammonia and glutamine metabolism in several organs has received considerable attention. However, virtually no attention has been paid to the interaction between these organs during liver failure. Especially, no attempts have been made to quantify the exchange of nitrogen and specifically ammonia and glutamine between organs. 
Therefore, the principal aim of this thesis was to study nitrogen metabolism during acute and chronic liver failure with special emphasis on the mass flux of ammonia and glutamine between several organs. As an introduction to the experimental work underlying this thesis, a review of the relevant literature will be given. First, some biochemical aspects of ammonia and glutamine, as well as the key reactions in their synthesis and breakdown will be discussed. Next, the role of several organs in ammonia and glutamine metabolism in the physiological situation will be addressed, followed by an outline of ammonia and glutamine metabolism during liver failure according to prevailing hypotheses. Finally, a brief account of the metabolic consequences of bile duct ligation will be given, because common bile duct ligation was used as part of one of the animal models in this thesis.

\section{AMMONIA}

Ammonia is a toxic nitrogenous product of many degradation reactions in nitrogen metabolism (3). 'Ammonia' in this thesis refers to total ammonia concentrations, i.e. $\mathrm{NH}_{3}+\mathrm{NH}_{4}{ }^{+}$. Ammonium is formed from ammonia and vice versa in the equilibrium reaction $\mathrm{NH}_{3}+\mathrm{H}^{+}$ $\mathrm{NH}_{4}{ }^{+}(\mathrm{pKa}=9.05-9.26$ (4-6)). At physiological $\mathrm{pH}$ the overwhelming majority (99\%) is in the form of ammonium $\left(\mathrm{NH}_{4}{ }^{+}\right)(3,7)$. The ammonia $\left(\mathrm{NH}_{3}\right)$ form is gaseous and lipophilic and can easily diffuse across cell membranes, whereas the ammonium ion is non-diffusible and can only be transported by carrier-mediated processes $(3,6,8,9)$. Since only the gaseous $\mathrm{NH}_{3}$ form is freely diffusible across cell membranes, this form probably is important in ammonia toxicity, because $\mathrm{NH}_{3}$ transport across membranes is profoundly influenced by the $\mathrm{pH}$ difference across membranes (10). In this context, it must be emphasized that the permeability of membranes for $\mathrm{NH}_{3}$ depends on the lipid content of membranes as well as on the magnitude of their surface area (6). Thus, membranes having a high protein content are less permeable to $\mathrm{NH}_{3}$; similarly, if, as e.g. in kidney tubular cells, the apical surface area of a cell is considerably smaller than the basolateral surface, then the apical membrane is less permeable (6).

Although ammonia is an important participant in several biochemical pathways, it is toxic to the organism at elevated levels $(3,11)$. Therefore, it must be converted to a non-toxic compound and several pathways serve this purpose. In most mammals, this takes place mainly in the liver by the synthesis of urea in the urea-cycle (ornithine cycle) (for review, see $(2,3)$ ). However, during liver failure diminished urea synthesis capacity and portasystemic shunting $(1,2,12)$ will cause that the main route of ammonia detoxification (urea synthesis) is impaired and/or bypassed, leading to hyperammonemia (2). In this situation the synthesis of glutamine (see below) from equimolar amounts of glutamate and ammonia becomes the most important altemative, although temporary, pathway for ammonia detoxification $\left(1,3,13^{\mathrm{a}}, 13^{\mathrm{b}}\right)$.

\section{GLUTAMINE}

Glutamine is a non-toxic, non-essential amino acid, consisting of a five-carbon chain and two nitrogen residues. One of these nitrogen residues is in the $\alpha$-position (the amino-N: a 
common feature of all amino acids), the other is in the $\delta$-position (the amide-N). From all amino acids that can be incorporated in protein, glutamine is the most abundant amino acid in the body: it has the highest plasma concentration of all amino acids, and it constitutes approximately $50 \%$ of the whole body free amino acid pool $(14,15)$. Glutamine is commonly available in many dietary sources (14). It serves as an obligatory fuel for intestinal (16-18) and other rapidly dividing cells e.g. the immune system $(3,14)$, and plays an important role in the regulation of acid/base balance by providing the most important substrate for renal ammoniagenesis $(6,19)$. Furthermore, it provides precursors for nucleic acid biosynthesis (20), protein synthesis (3) and may be involved in the regulation of protein synthesis $(20,21)$. Also, the spontaneous breakdown of glutamine residues in proteins has recently been suggested to serve as a biological clock regulating the spontaneous life span of protein: glutamine deamidation would lead to loss of protein structure thereby determining the intrinsic lifetime of specific proteins (22).

Glutamine depletion, as for example in postoperative surgical patients and during sepsis, has been suggested to lead to an energy deficit in the intestinal mucosal cells, adversely affecting mucosal barrier function. This would facilitate bacterial translocation $(23,24)$, enhance bacteremia and possibly contribute to the pathogenesis of multi-organ failure $(15,25)$. Similarly, it could contribute to immune system malfunction (26). Since glutamine is not present in parenteral nutritional solutions, glutamine addition to these solutions, or enteral glutamine administration simultaneous with parenteral feeding has received considerable attention in order to develop methods to support intestinal energy metabolism and mucosal barrier function in trauma patients $(25,27-29)$. In view of its presumed and partly proven importance as a respiratory fuel for the gut and as an interorgan nitrogen carrier in several disease states, glutamine is sometimes referred to as a 'conditionally' essential amino acid (14).

\section{ENZYMES RELATED TO AMMONIA AND GLUTAMINE METABOLISM}

Several enzymes are of crucial importance in ammonia and glutamine metabolism. This thesis mainly focuses on the pathophysiology of ammonia and glutamine metabolism during liver failure, when urea synthesis capacity is reduced and altemative pathways of ammonia detoxification become increasingly important. Therefore, only those enzymes potentially playing a role in these altemative pathways will be discussed here (for a review on urea cycle function, see (2)).

Glutamine synthetase (EC 6.3.1.2) catalyzes the synthesis of glutamine from equimolar amounts of glutamate and ammonia:

Equation 1: $\quad \begin{aligned} \text { L-glutamate } & +\mathrm{ATP}^{4-}+\mathrm{NH}_{4}{ }^{1+} \rightarrow \text { L-glutamine } \\ & +\mathrm{ADP}^{3-}+\mathrm{P}_{\mathrm{i}}{ }^{2-}+\mathrm{H}^{1+}\end{aligned}$

This is an energy consuming unidirectional reaction. Since glutamine is a non-toxic amino acid, this reaction constitutes a pathway of ammonia detoxification, that can function as an alternative for urea synthesis. Glutamine synthetase is a cytosolic enzyme. If the glutamine 
synthetase reaction must continuously detoxify ammonia, it must also continuously be furnished with glutamate. Several reactions have been described, that could yield glutamate.

Glutaminase (EC 3.5.1.2) catalyzes the reverse reaction of glutamine synthetase, which splits of the amide group of glutamine to yield equimolar amounts of ammonia and glutamate:

Equation 2: $\quad$ L-glutamine $+\mathrm{H}_{2} \mathrm{O} \rightarrow$ L-glutamate ${ }^{1-}+\mathrm{NH}_{4}{ }^{1+}$

Several types of glutaminase enzymes exist ( $\omega$-amidase, $\gamma$-glutamyl transferase, $\gamma$-glutamyl transpeptidase), the most important of them being intra-mitochondrial phosphate dependent glutaminase. Although this reaction evidently generates glutamate, it also generates ammonia. Thus, although it occurs in another compartment of the cell than the glutamine synthetase reaction, this would not help in detoxifying ammonia.

Glutamate dehydrogenase (EC 1.4.1.2) catalyzes the synthesis of glutamate from equimolar amounts of 2-oxoglutarate ( $\alpha$-ketoglutarate) and ammonia, as well as the reverse reaction:

Equation 3: 2-oxoglutarate ${ }^{2-}+\mathrm{NH}_{4}{ }^{1+}+\mathrm{NAD}(\mathrm{P}) \mathrm{H}+\mathrm{H}^{1+}+$ L-glutamate $^{1-}$

$$
+\mathrm{NAD}(\mathrm{P})^{1+}+\mathrm{H}_{2} \mathrm{O}
$$

This reaction can provide glutamate for the glutamine synthetase reaction. However, since it is a reversible reaction, it may just as well generate ammonia under certain circumstances (5).

Transamination reactions can also provide glutamate for the glutamine synthetase reaction $\left(3,30^{\mathrm{a}}\right)$. The transamination reactions mainly consist of two types. First, the aspartate amino transferase (ASAT (=SGOT); EC 2.6.1.1) reaction requiring aspartate and 2-oxoglutarate, producing glutamate and oxaloacetate:

Equation 4: $\quad$ aspartate $^{1-}+2-$ oxoglutarate ${ }^{2-} \approx$ oxaloacetate $^{2-}+$ glutamate $^{1-}$

and second, the branched chain amino acid amino transferase reactions (EC 2.6.1.42), leucine aminotransferase (EC 2.6.1.6) and valine-isoleucine amino transferase (EC 2.6.1.32) requiring BCAA's and 2-oxoglutarate producing glutamate and branched chain oxoacids (oxoleucine, 2 -oxoisovalerate) $\left(3,30^{\mathrm{a}}\right)$ :

Equation 5: $\quad \mathrm{BCAA}^{1 \cdot}+2$-oxoglutarate ${ }^{2-}=\mathrm{BCOA}^{2-}+$ glutamate $^{1-}$.

Carbon-dioxide fixation via pyruvate carboxylase (EC 6.4.1.1), finally, has been suggested as an alternative means of providing the carbon chains for glutamine synthesis, particularly in brain $\left(5,30^{b}\right)$ 


\section{GLUTAMINE AND AMMONIA METABOLISM IN THE PHYSIOLOGICAL SITUATION}

Intestines (Figure 1) The intestines contain high glutaminase activity and only little glutamine synthetase activity $(16,17,31)$. This enzyme distribution pattern makes of the intestines an organ mainly of glutamine breakdown.

Glutamine is one of the principal energy substrates of the large and especially the small intestine. Besides glucose, short chain fatty acids and ketone bodies, the intestines

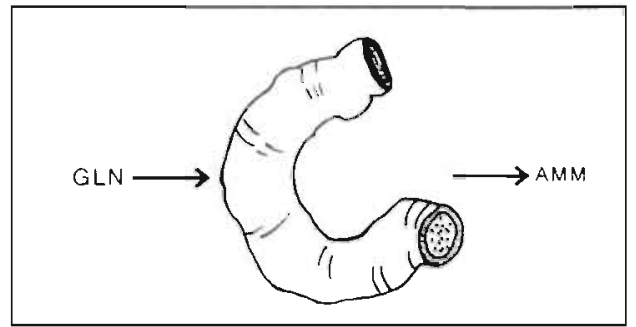

Figure 1: Intestinal ammonia and glutamine exchange: physiological role. take up glutamine in large quantities from either the blood-stream or the intestinal lumen $(16,17,32,33)$. Most of the glutamine consumed by the intestines is utilized in the small intestinal mucosa, constituting $75 \%$ of small intestinal weight (34). The large bowel utilizes much less glutamine than the small intestine, but instead uses other substrates e.g. glucose, short chain fatty acids and ketone bodies (35). Luminal or blood derived glutamine uptake by the rat small bowel is a concentrationdependent process, that reaches a maximum at plasma concentrations of approximately 600 $\mu \mathrm{M}(17)$.

Intestinal glutamine uptake and subsequent metabolism can account for approximately $60 \%$ of total ammonia release from the portal drained viscera (35). Small bowel mucosa (and to a much lesser extent large bowel (35)) produces ammonia by converting blood-borne as well as luminal glutamine to ammonia and glutamate $(17,32,35-37)$. The latter is transaminated to alanine and to a lesser degree to other amino acids and organic acids $(16,17)$. Apart from this glutamine metabolism-derived ammonia, colonic bacteria, present in the gut lumen, produce ammonia by splitting urea and possibly amino acids $(35,36,38)$.

These two processes, namely glutamine breakdown and urea splitting, make the intestines a major ammonia producing organ. It should again be emphasized that ammonia is a toxic 'end'-product of nitrogen metabolism. The ammonia and amino acids are released into the portal blood and transported to the liver (16), where ammonia and alanine nitrogen enter the urea cycle, whereas the alanine carbon skeleton is utilized in hepatic

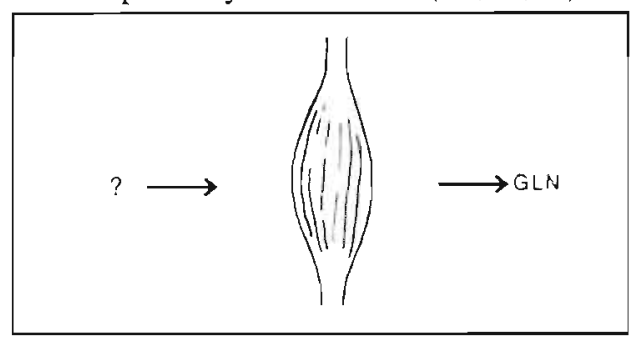

Figure 2: Skeletal muscle ammonia and glutamine exchange in the physiological situation. gluconeogenesis (39). Thus, it is only by virtue of the localization of the liver immediately downstream of the vascular bed of the intestines, that the intestines can utilize without adverse effects for the organism a fuel that produces a potentially harmful end-product.

Muscle (Figure 2) Glutamine synthetase activity in skeletal muscle is low (31), and it is mainly by virtue of its mass relative to other glutamine synthetase containing organs, that muscle is one of the principal glutamine synthesizing organs $\left(13^{a}, 40\right)$. Although skeletal muscle has long been thought to contain no glutaminase activity (21), recently evidence has 
accumulated, that skeletal muscle does contain glutaminase activity in several animal species (41-43). However, there are several methodological difficulties in this field, since it has been suggested that the glutaminase activity detected in skeletal muscle may be attributable to infiltrating inflammatory cells (44).

The role of skeletal muscle in ammonia metabolism has not yet been conclusively established. It has been stated that ammonia can be taken up by or released from skeletal muscle. Skeletal muscle ammonia release has been suggested to occur during exercise, probably related to purine nucleotide cycle activation $(18,45)$. Ammonia uptake by skeletal muscle has been proposed to occur in several hyperammonemic states as e.g. in rats during ammonia infusion $(46,47)$, humans during valproate-induced hyperammonemia $(48,49)$, and dogs $(50,51)$, but also in healthy human volunteers $(48,49)$ and normal control rats $(46)$. This ammonia uptake has been hypothesized to lead to glutamine synthesis in the glutamine synthetase reaction (equation 1), resulting in an augmentation of the skeletal muscle free glutamine pool and glutamine release from skeletal muscle $\left(1 ; 13^{a}, 52\right)$.

The discussion, whether ammonia uptake by skeletal muscle will always lead to glutamine release and whether this glutamine release is stoichiometric to ammonia uptake, is confounded by contrasting literature reports. Thus, enhanced ammonia uptake by skeletal muscle was found in vivo upon ammonia infusion in intact rats $(46,47,53)$, monkeys $(50)$, dogs (50,51) and pigs (54). This ammonia uptake enhanced skeletal muscle glutamine release only in some of these studies $(50,53,54)$, whereas glutamine release remained unchanged in others (46,51). Ammonia uptake either exceeded (54) or balanced the enhanced glutamine release (50). These literature data demonstrate that stoichiometry cannot consistently be shown. An explanation might reside in the fact that most of these experiments were acute hyperammonemia experiments and do not take into account the changes in tissuc concentrations. In acute hyperammonemia experiments, steady state is per definition lacking and part or all of the ammonia could be trapped in augmented tissue ammonia and amino acid ( $\approx$ glutamine) pools $\left(13^{b}\right)$. This need not necessarily induce a release of these amino acids. In chronic hyperammonemia experiments the situation is different because hyperammonemia is continuously present to the same extent. Therefore, under these steady state hyperammonemia conditions ammonia trapping in tissues is probably not an important phenomenon.

It is generally accepted that skeletal muscle is an organ of net glutamine release in the physiological situation $(18,55)$ as well as in various disease states $(18,40,41,55)$. Skeletal muscle contains $70-80 \%$ of the total body free amino acid pool and glutamine constitutes $60 \%$ of this muscle pool (excluding taurine, which is not incorporated in protein) (15). Thus, apart from being potentially explainable by enhanced glutamine synthe-

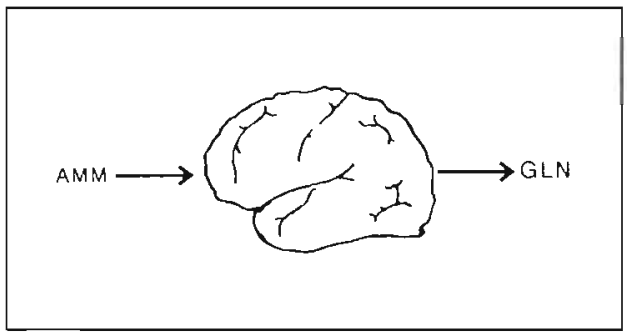

Figure 3: Cerebral ammonia and glulamine metabolism in the normal situation. sis, any glutamine release from skeletal muscle can also be caused by changes in muscle protein turnover and/or release from the free glutamine pool $(21,42,56)$. In this context, it has been shown that the composition of the amino acids released from muscle due to proteolysis (57) is not representative of skeletal muscle protein composition (58). Glutamine constitutes approximately $30 \%$ of the amino 
acids released from skeletal muscle, although it constitutes only $5 \%$ of skeletal muscle protein $(42,56,58,59)$. For this reason, glutamine is commonly viewed as a carbon and nitrogen carrier from skeletal muscle to splanchnic organs $(15,58)$. Any attempt to study muscle glutamine synthesis from blood-bome ammonia must take into account the commonly observed muscle glutamine release during catabolic states $(15,21,41)$.

Brain (Figure 3) Brain contains appreciable amounts of both glutamine synthetase $(5,31,60)$ and glutaminase activity $(5,61-63)$. These two enzymes are, however, to a considerable extent compartmentalized in brain. This is not only so on the sub-cellular level (which is the case in all other organs, since glutamine synthetase is a cytosolic and glutaminase an intra-mitochondrial enzyme), but also on the cellular level. Thus, the astrocytes contain most of the total brain glutamine synthetase activity $(60,64)$, only maximally $20 \%$ being localized in the neurons, and the neurons contain virtually all brain glutaminase activity (5).

The normal brain is generally viewed as an organ of ammonia uptake and glutamine release $\left(5,13^{\mathrm{a}}, 65\right)$. Ammonia uptake has been reported in healthy human volunteers $(48,49)$ and in some, but not all animal species (5). Despite a tremendous amount of research, ammonia uptake by the normal rat brain has never been confirmed (5) and reports confirming net glutamine release from the brain of normal rats are scarce (52).

Kidney (Figure 4) Both glutaminase and glutamine synthetase are present in the kidneys (66). Therefore, the kidneys are probably capable of both synthesizing and degrading glutamine. Conflicting results have been reported $(67,68)$, but the preponderance of evidence suggests that glutamine is taken up by the normal rat $(55,66,69-75)$ and human $(49,76)$ kidney. Glutamine is the main substrate for renal ammoniagenesis $(6,77)$. The

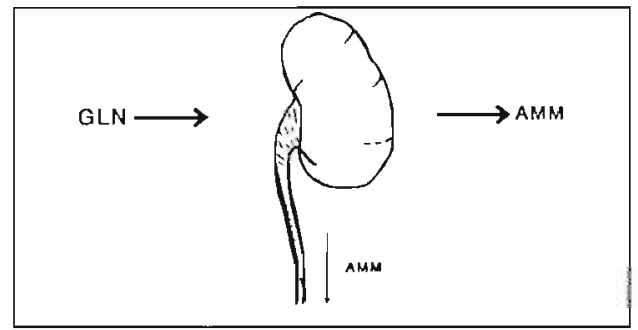

Figure 4: Physiological role of the kidney in glutamine and ammonia handling. glutamine taken up by the kidney is metabolized predominantly in the intramitochondrial phosphate-dependent glutaminase reaction, and to a much lesser degree by the action of the membrane-bound $\gamma$-glutamyl transferase $(6,73)$. Thus, total renal ammoniagenesis is mainly related to glutaminase activity. The ammonia produced by the kidney is either excreted in the urine or released into the renal vein $(6,19,67)$.

Vinay et al. (67) found that in the physiological situation, the kidneys excrete only minor (as compared to urea nitrogen excretion) amounts of ammonia in the urine. Only approximately $30 \%$ of total ammonia production in the kidneys is released into the urine in the normal rat, the remainder is released into the renal vein $(6,67)$. The same values of $70 \%$ released into the renal vein and $30 \%$ into the urine are found in control rats used in acidosis studies, to whom $\mathrm{NH}_{4} \mathrm{HCO}_{3}$ is administered $(70,71)$. These figures clearly illustrate that the normal kidney is an organ of net ammonia addition to the body pools. During chronic acidosis, however, the situation reverses because total renal ammoniagenesis is enhanced and $70 \%$ of this enhanced amount is excreted in the urine to dispose the acid load $(6,19,70$, $71,73)$. It is interesting to note that in two well-documented studies of chronic acidosis $(70,71)$ in which $\mathrm{NH}_{4} \mathrm{Cl}$ and $\mathrm{NH}_{4} \mathrm{HCO}_{3}$ were administered to obtain acidotic and control rats respectively, this enhanced renal ammoniagenesis and urinary ammonia excretion was 
observedi at elevated arterial ammonia levels. This observation could imply that arterial ammonia itself is important in regulating urinary ammonia excretion, rather than $\mathrm{pH}$ alone.

It still is not clear whether both nitrogen moieties of glutamine always serve renal ammoniagenesis (reviewed in (6)). The amide moiety always serves ammoniagenesis in the glutaminase reaction, but subsequently three possible fates for the resulting glutamate remain. First, the glutamate can be released into the renal vein $\left(78^{\mathrm{a}}\right)$. Alternatively, it can be used in transamination reactions, yielding predominantly alanine (6). Finally, the amine moiety can be split of in the glutamate dehydrogenase reaction, yielding $\alpha$-ketoglutarate (the carbon skeleton of glutamine), which can be oxidized in the TCA cycle through the PEPCK pathway $(6,19,73)$. After being released into the blood stream $(70)$, alanine can be taken up by the liver, where the carbon skeleton can be used in hepatic gluconeogenesis, the nitrogen for e.g. in urea synthesis (71).

Renal glutamine and ammonia metabolism plays an important role in acid-base homeostasis $(6,19)$. Glutamine breakdown and subsequent ammonia excretion in the urine has been suggested to be an efficient means of excreting protons and generating new bicarbonate $(6,19)$. However, recently considerable debate has arisen concerning this subject, because some authors have suggested that ammonia excretion in the

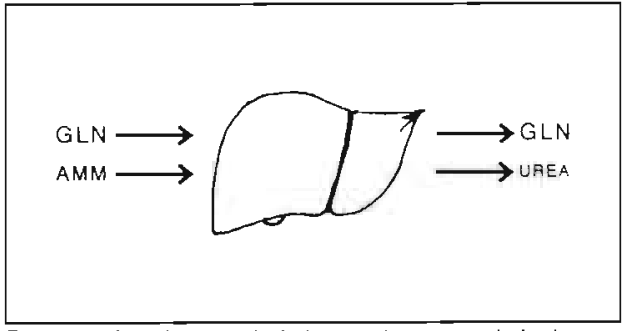

Figure 5: Hepatic control of nitrogen homeostasis in the normal situation. urine does not represent proton excretion, but merely represents the excretion of the nitrogen moiety of amino acids $\left(78^{\mathrm{b}}\right)$. In this respect the role of urea synthesis as a bicarbonate removing and $\mathrm{pH}$-regulating modality has been stressed $\left(2,78^{\mathrm{b}}\right)$. Nonetheless, the effects of acute and chronic metabolic acidosis on kidney ammonia and glutamine metabolism have been extensively studied (55,69-73). During chronic metabolic acidosis, glutamine extraction by the kidney is increased (69-71) and ammonia release into the urine also increases at the expense of urea $(66,71)$. However, the total amount of nitrogen released in the urine as ammonia plus urea remains constant $(66,71)$. To our knowledge no data are available concerning the effects of chronic alkalosis on renal glutamine and ammonia metabolism.

Liver (Figure 5) The liver contains both glutaminase and glutamine synthetase activity $(31,66,71,72)$. These enzymes have a zonated distribution pattern in the liver (79). Glutamine synthetase activity is restricted to a small hepatocyte population immediately surrounding the hepatic venules, whereas glutaminase activity is mainly located in the periportal zone $\left(79,80^{\mathrm{s}}\right)$.

The glutaminase activity in the periportal zone generates ammonia, which, in addition to the ammonia taken up from portal drained viscera-derived blood, has a feed-forward effect on hepatic urea synthesis $(71,72,79)$. Any ammonia escaping detoxification in the ureacycle is trapped in the perihepatic-venous glutamine synthetase containing cells (not containing urea cycle enzymes (79)) and undergoes alternative detoxification by glutamine synthesis (79). Thus, the liver is capable of both synthesizing and degrading glutamine. Whether net production or consumption of glutamine by the liver occurs, is dependent on among others tissue ammonia concentrations and $\mathrm{pH}$ (72). Ammonia activates glutaminase, whereas a fall in $\mathrm{pH}$ is associated with decreased glutaminase flux and increased glutamine 
production (72). Also, protons 'consume' bicarbonate, reducing the bicarbonate available for urea synthesis. Therefore, during acute and chronic acidosis, the liver becomes an organ of enhanced net glutamine release $(66,69,71,72)$.

Without any doubt, the liver is an organ of net ammonia uptake (see e.g. $(66,71)$ ). Ammonia taken up by the liver is detoxified mainly in periportal hepatocytes in the urea

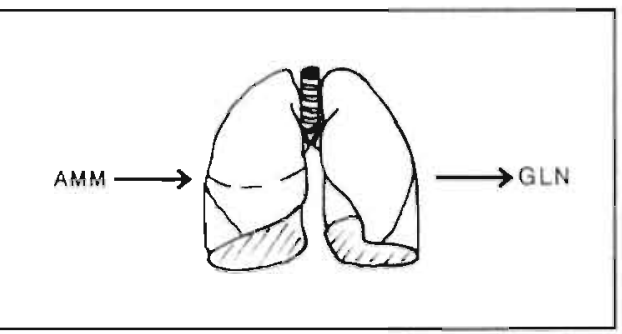

Figure 6: Presumable role of the lungs in ammonia and glutarmine exchange.

cycle and released into the hepatic vein as urea (79), which is subsequently excreted into the urine. Part of the urea synthesized, however, participates in an enterohepatic cycle, since colonic bacteria generate ammonia by splitting luminal urea $(35,36,38)$. Part of the ammonia, not detoxified in urea synthesis (a low affinity, high capacity detoxification pathway (79)), is trapped in the perivenous hepatocytes and incorporated in glutamine in the glutamine synthetase reaction (high affinity system) (79).

In the physiological situation, periportal glutamine breakdown and perivenous glutamine synthesis are probably of equal magnitude and therefore the liver glutamine balance is close to zero (79). In view of the high hepatic activities of glutaminase as well as glutamine synthetase $(31,66)$ and the way these enzymes are regulated $\left(79,80^{\mathrm{a}}\right)$, howev$\mathrm{er}$, the liver can rapidly respond to changes in systemic ammonia levels and/or acid-base

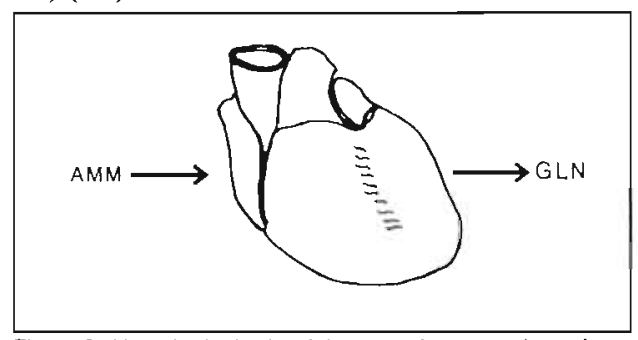

Figure 7: Hypothetical role of the heart in ammonia and glutamine exchange. status by changing from glutamine uptake to release $(66,69,71,72,79)$. Of special interest in this respect is the sensitivity of hepatic glutaminase to ammonia and $\mathrm{pH}$. Hepatic glutaminase, in contrast with other types of glutaminase, that are inhibited by high ammonia levels (3), is activated by ammonia $\left(72,79,80^{\mathrm{a}}\right)$ and in fact the presence of ammonia is essential for the action of the enzyme $\left(80^{\mathrm{b}}\right)$. The inhibition of hepatic glutaminase at lower $\mathrm{pH}$ values appears to be mediated by an altered affinity of the enzyme without any effect on the $V_{\max }$ $\left(80^{b}\right)$

Lung (Figure 6) Recently, considerable attention has been paid to the role of the lung in glutamine and ammonia homeostasis (81-84). Currently available evidence suggests that the normal $(81)$ as well as the injured $(82,84)$ lung is an organ of net glutamine release. Also, ammonia uptake by the lung in septic patients has been observed (84).

However, the stoichiometry between glutamine release and ammonia uptake could not be demonstrated, and it still is unclear what type of cells are responsible for pulmonary glutamine release (84). Although pulmonary glutamine release and ammonia uptake would be compatible with the presence of glutamine synthetase activity in lung tissue $(31,81)$, the exact role of the lungs in ammonia and glutamine homeostasis remains to be elucidated.

Heart (Figure 7) We are not aware of any literature studies conceming the role of the heart in ammonia and glutamine exchange. Considering the enzymatic properties of myocardial cells $(31,43)$, the heart could contribute to glutamine production as well as breakdown. In the past, glutamine synthesis was demonstrated in the cayman heart (85). In 
view of its limited weight (86) but abundant perfusion $(87,88)$, it is difficult to estimate the contribution of the heart to interorgan ammonia and glutamine exchange.

Immune system (Figure 8) Evidence is accumulating from in-vitro and in-vivo studies, that cells from the immune system consume glutamine and produce ammonia $(3,26,44,89)$. The importance of glutamine and ammonia metabolism in interorgan nitro-

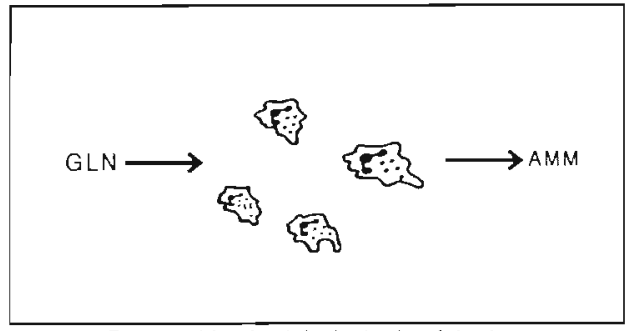

Figure 8: Presumable physiological role of the immune system in glutamine and ammonia exchange. gen exchange, however, remains to be established.

\section{LIVER INSUFFICIENCY: CONSEQUENCES FOR GLUTAMINE METABOLISM}

Liver (Figure 9) Liver disease, whether acute or chronic, leads to impaired hepatic metabolism of various metabolites, among others ammonia $(1,2,7)$. Several studies have demonstrated diminished urea synthesis and glutamine synthesis capacity during liver failure $\left(12,79,80^{u}, 90\right)$. This reduced ammonia detoxification combined with the usually existing portasystemic shunts have been proposed to contribute to systemic hyperam-

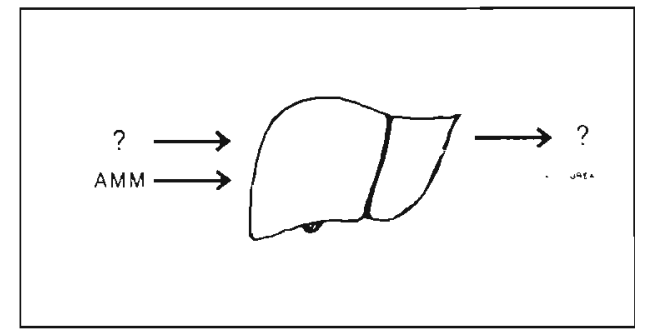

Figure 9: Hepatic nitrogen homeostasis during liver failure: little is known about glutamine handling. monemia $(1,2,7)$. Very little literature has appeared on hepatic ammonia and glutamine metabolism during acute or chronic liver failure. Interestingly, flux through the glutaminase reaction was shown to be increased 4- to 6-fold in patients with chronic liver failure $\left(79,80^{\mathrm{a}}\right)$. Kaiser et al. $\left(80^{\mathrm{a}}\right)$ suggested that this would lead to enhanced ammonia formation, acting as a compensatory mechanism for maintenance of a life-compatible urea-cycle flux in a situation of reduced urea synthetic capacity. However, despite its pivotal role in nitrogen metabolism and ammonia detoxification, virtually no research has been done on the role of the diseased liver in inter-organ nitrogen transport and ammonia and glutamine exchange. This is particularly true for experimental animal research and is probably caused by the difficulty of reliably measuring net hepatic metabolite exchange in general, and in animals with liver disease in particular.

Intestines (Figure 10) During liver failure, ammonia derived from intestinal glutamine metabolism is only partly taken up from the portal vein and cleared by hepatic urea synthesis. Thus, as a consequence of hepatocellular failure and portasystemic shunting, gutderived ammonia escapes hepatic clearance, resulting in systemic hyperammonemia. The elevated glutamine levels encountered during liver failure (52) could enhance intestinal glutamine consumption and thus ammonia production, because intestinal glutamine uptake is concentration dependent up to arterial concentrations of $600 \mu \mathrm{M}$ (17).

High ammonia levels have been repeatedly implicated in the pathogenesis of hepatic encephalopathy (1,5,91), a neuropsychiatric syndrome often accompanying hepatic failure (92). Hepatic encephalopathy is an important cause of morbidity and mortality in patients 
with acute and chronic liver failure $(92,93)$. Hyperammonemia and hepatic encephalopathy are often precipitated by gastrointestinal hemorrhage (or other accidental high protein loads) (91), or protein overload of endogenous origin during catabolic illness (92).

The association between high protein loads, hyperammonemia and hepatic encephalopathy in patients with hepatic failure has led to the generally accepted idea that enhanced intestinal ammonia liberation is an

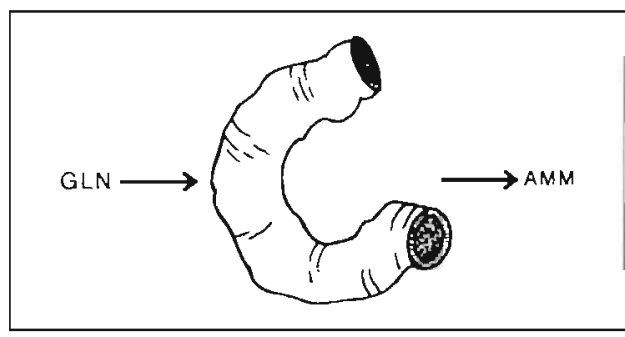

Figure 10: Intestinal glutamine and ammonia metabolism during liver failure: information about quantitative changes is lacking. important factor in the pathogenesis of hepatic encephalopathy $(1,91,94)$. Consequently, standard therapies focus on removal of intestinal contents, accelerating intestinal transit time by cathartic agents, reducing protein loads, lowering systemic ammonia levels by acidification of intestinal contents with lactulose and reducing bacterial flora with poorly resorbable antibiotics (for review see $(91,94)$ ). Insight in the role of the intestines in interorgan nitrogen exchange during several hyperammonemic states could therefore be valuable in developing new therapeutic strategies in the treatment of the complications of liver failure.

Muscle (Figure 11) Unidirectional ammonia uptake by human skeletal muscle has been shown by tracer techniques during hyperammonemia caused by liver failure $\left(13^{\mathrm{a}}\right)$. As stated before, net ammonia uptake by skeletal muscle has been demonstrated by measurements of arteriovenous (AV) differences in fasted healthy human volunteers across the leg $(45,48,49,95)$, but not across the forearm $(40,65)$. In fasted humans with

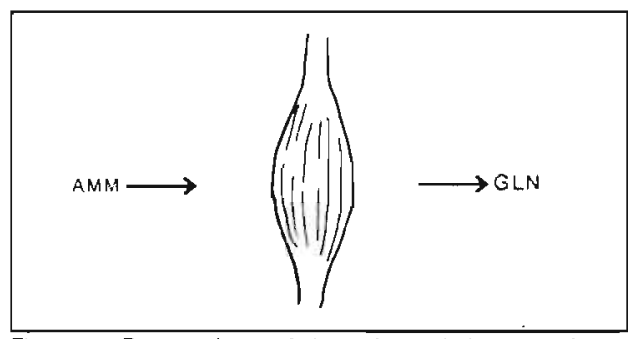

Figure 11: Proposed role of skeletal muscle in ammonia and glutamine exchange during liver failure.

liver disease, ammonia was taken up by the leg $(95,96)$ and forearm $(40,65)$. Similarly, net ammonia uptake by muscle was demonstrated in fasted healthy dogs $(50,51)$ and monkeys (50), as well as across the hindquarter in fed (97) and fasted normal rats (46). In some of these studies $(40,49,50,65,95)$ AV concentration differences across skeletal muscle were found to correlate with arterial ammonia levels: the higher the arterial levels, the greater ammonia uptake. Fine (51), however, could not confirm this correlation in an ammonia infusion study in dogs. Nonetheless, the considerable difference between the large unidirectional uptake by muscle and the only small AV differences for ammonia across muscle have been interpreted as evidence that muscle continuously uses and produces ammonia $\left(13^{\mathrm{a}}\right)$. Also, the fact that these AV differences are small probably indicates that muscle ammonia uptake is only important because skeletal muscle constitutes over $40 \%$ of body mass. This also means that muscle wasting during liver disease could reduce the potential role of muscle in ammonia detoxification $\left(13^{\mathrm{a}}\right)$. Muscle ammonia uptake has been suggested to be enhanced during liver failure and this enhanced muscle ammonia uptake and subsequent glutamine synthesis has been hypothesized to constitute the major altemative means of ammonia detoxification in the absence of a healthy liver $\left(13^{\mathrm{a}}, 40,65,98\right)$. However, the quantitative role of muscle in ammonia detoxification in vivo is unclear $(16,40,47,50,53$, 
98). Moreover, as outlined above, this hypothesis is mainly based on observations in humans with chronic liver failure and it is unclear whether this is applicable to all hyperammonemic states. Finally, as discussed before, the relation between ammonia uptake and glutamine release remains to be elucidated.

Brain (Figure 12) Unidirectional ammonia uptake by the brain has been observed in normal rats and humans using tracer techniques $\left(13^{\mathrm{a}}, 99\right)$. Similar observations were made in these species during hyperammonemia, either associated with liver disease or induced by portacaval shunting or urease treatment $\left(13^{\mathrm{a}}, 100\right)$. Net ammonia uptake by the brain during hyperammonemia has been demonstrated by AV concentration differ-

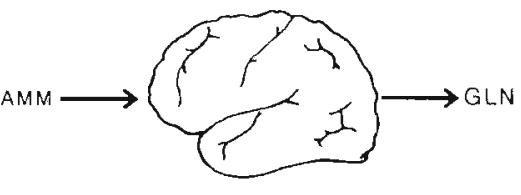

Figure 12: Cerebral ammonia and glutamine metabolism during liver failure: current hypothesis. ence measurements in valproate-treated humans $(48,49)$ and cirrhotic patients $(65,98)$. Also, net ammonia uptake by the brain has been confirmed in rats made acutely hyperammonemic by intraperitoneal ammonium-acetate injections (101) and in 8 weeks portacaval shunted rats, but not in 4 weeks portacaval shunted rats (52). Thus, although cerebral ammonia uptake probably occurs during hyperammonemia, this has not been confirmed for all animal species and certainly not for all different causes of hyperammonemia in experimental animals. Finally net ammonia uptake by the brain has never been proven in normal rats (5) and in humans conflicting reports have been published concerning this subject $(48,49,65)$. In analogy to skeletal muscle it has been proposed that brain continuously uses and produces ammonia, the ultimate result being a net uptake fraction of 4.8 to $11.0 \%\left(13^{\mathrm{a}}, 99\right)$.

Tracer studies using ${ }^{13} \mathrm{~N}$-ammonia show that in the normal rat brain ammonia is almost instantaneously incorporated for over $95 \%$ in the amide group of glutamine $\left(t_{1 / 2}<3 \mathrm{sec}\right)(99)$, suggesting that cerebral ammonia detoxification is predominantly by glutamine synthetase $(5,99,100)$. Glutamine synthesis mainly takes place in a small $(20 \%)$ compartment of the brain, in which rapid glutamate turnover occurs (probably the astrocytes $(5,60,99,100)$ ).

In the hyperammonemic rat brain, tracer amounts of ammonia are less rapidly incorporated in glutamine than in the normal brain $\left(t_{1 / 2}=9 \mathrm{sec}\right)(100)$. The hyperammonemic brain has been shown to have a higher glutamine content than the normal rat brain $(11,101-104)$ and some of these authors also found a lower content of glutamate during hyperammonemia (102-104). Such observations could be explained by an augmented enzymatic machinery incorporating ammonia at a lesser velocity, but there is evidence that hyperammonemia does not lead to enzyme induction in the brain (5), and glutamine synthetase activity in whole brain was found to be unchanged after 14 weeks of portacaval shunting (100). Glutamine synthetase activity in the cerebral cortex of the rat was reported to be decreased following portacaval anastomosis $(105,106)$.

Ammonia probably plays a key role in the pathogenesis of hepatic encephalopathy $(1,5,91)$. Although there is a fairly good correlation between tissue ammonia concentrations and the degree of encephalopathic changes $(5,107)$, such a correlation cannot easily be demonstrated for plasma ammonia levels and degree of encephalopathy. This has led to the general belief that there must be other factors acting synergistically with ammonia in determining neurological status (1). Several hypotheses concerning the pathogenesis of the neurological dysfunction have been studied (for review, see (91): high energy phosphate 
depletion $(11,102,108)$, Krebs-cycle intermediate depletion (2-oxoglutarate) $(98,101,102)$, neurotransmitter changes (for reviews, see $\left(5,109^{a}\right)$ ) or an alteration in the neuronal membrane potential by chloride concentration changes (reviewed in (1)). Despite substantial evidence that high energy phosphate depletion as well as an increase in the concentration of false neurotransmitters (octopamines) are not causal factors, the pathogenesis of hepatic encephalopathy has not yet been conclusively elucidated $(1,91)$. In this context, the role of brain edema as a consequence of cellular swelling induced by intra-cellular glutamine accumulation in the astrocytes remains to be established $\left(109^{\mathrm{b}}\right)$.

The overall net effect of the above described cerebral ammonia uptake and detoxification processes is the formation of glutamine from ammonia and glutamate. The production of glutamine could cause the often observed decreased glutamate concentrations in hyperammonemic brains (102-104). As glutamate probably is the most important excitatory neurotransmitter in the central nervous system $(5,107)$, an imbalance in

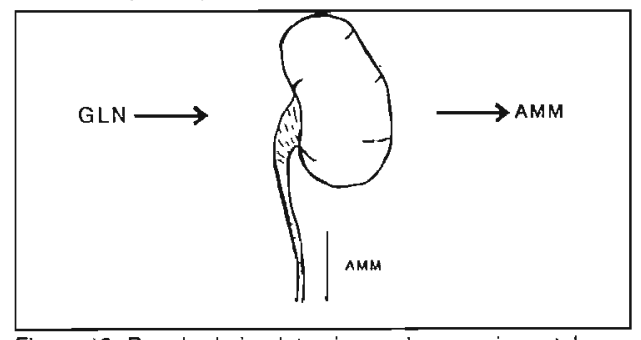

Figure 13: Renal role in glutamine and ammonia metabolism during liver failure: quantitative intormation is lacking. excitatory-inhibitory transmitters favoring inhibition could ensue, which could contribute to the pathogenesis of hepatic encephalopathy (91). The glutamine will probably at least partly be released into the circulation, thus leading to carbon skeleton loss from the brain $(5,52)$. Although, in view of their respective relative masses, brain glutamine synthetic capacity should be negligible compared with that of skeletal muscle, the contribution of the brain glutamine efflux to blood glutamine concentrations remains to be elucidated.

Kidneys (Figure 13) Despite its central role in waste nitrogen excretion during health and disease, virtually no research has been performed concerning the role of the kidney in inter-organ nitrogen exchange and ammonia excretion during liver disease and hyperammonemia. This is strange to some extent, since the association between liver and kidney is forcibly illustrated by the often fatal hepatorenal syndrome, sometimes accompa-

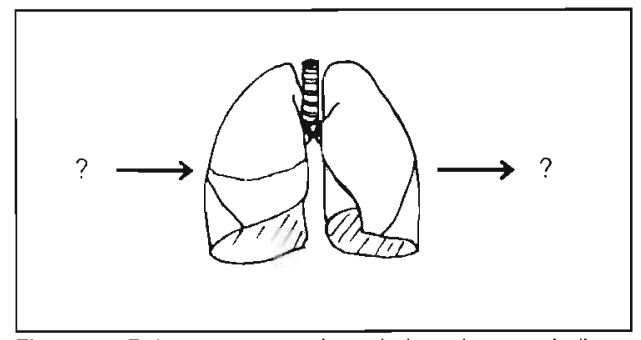

Figure 14: Pulmonary ammonia and glutamine metabolism during liver tailure: no information available. nying liver disease (92). In addition, renal glutamine and ammonia metabolism are intimately linked to acid base regulation, since ammonia has been suggested to be important in proton excretion in the urine $(6,19)$, and acidosis enhances renal glutamine uptake and urinary ammonia excretion (66,69-71). Finally, it is well-known from clinical practice that hepatic coma is often accompanied by chronic respiratory alkalosis (92). This has led some investigators to assess the effect of hyperventilation on ammonia exchange across the kidney (96). In this study it was demonstrated that ammonia release into the renal vein increased after hyperventilation in cirrhotic patients suggesting that hyperventilation impairs renal urinary ammonia excretion. However, the latter was not measured in that study (96).

In the past high glutamine and ammonia concentrations were suggested to favor renal ammonia excretion $(95,110-114)$. It was shown that the kidney releases ammonia into the circulation in cirrhotic patients $(95,96,115)$. This renal ammonia release into the circulation 
decreased at elevated ammonia concentrations (95). Similarly, artificially elevated arterial ammonia levels in human volunteers induced the kidney to take up ammonia from the circulation and to increase urinary ammonia excretion (112). A problem of these studies is that most of them do not provide data on renal glutamine metabolism. Thus, recent literature conceming renal ammonia and glutamine metabolism during liver disease in humans and especially in experimental animals is not available. We are aware of only one report concerning simultaneous measurement of renal ammonia and glutamine metabolism during liver failure in rats (116), in which portal vein strictures were used as a model of liver failure and portasystemic shunting.

Lungs (Figure 14) No reports have appeared in literature concerning ammonia and glutamine metabolism by the lung during acute or chronic liver failure. Considering the enzymatic characteristics of lung tissue, ammonia uptake and glutamine release during liver failure could be postulated $(31,81)$.

Heart (Figure 15) To our knowledge, no reports have appeared in literature concerning ammonia and glutamine metabolism by

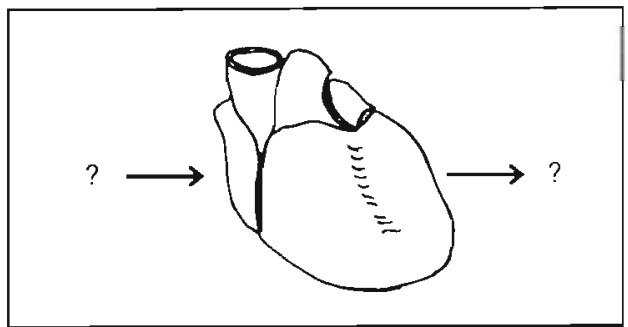

Figure 15: Cardiac ammonia and glutamine metabolism during liver failure: no information available. the heart during liver failure. Considering the enzymatic characteristics of cardiac tissue, it is extremely difficult to predict the role of the heart in ammonia and glutamine metabolism during liver failure $(31,43)$.

Immune system (Figure 16) In vitro studies showed that the effect of ammonia on protein synthetic and cell replication parameters in mice spleen depends on the dose of ammonia administered $(117,118)$. At low ammonia levels, these parameters are enhanced, whereas at elevated levels they are depressed. In rabbits, hyperammonemia induced by repeated urease injections led to leukopenia (119). These observations do suggest that hyperammonemia affects im-

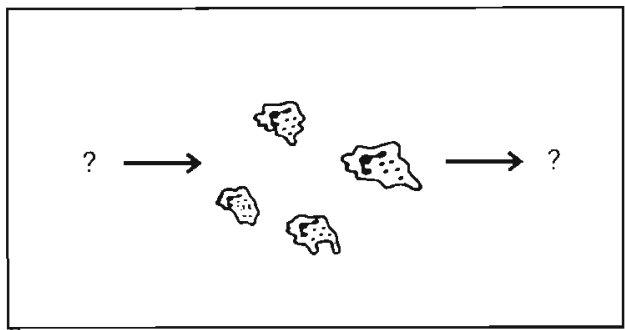

Figure 16: Ammonia and glutamine metabolism by the immune system during liver failure: no information available. mune system function, but it is not possible to predict what may be the effect on ammonia and glutamine metabolism by the immune system during liver failure.

\section{AMMONIA AND GLUTAMINE METABOLISM DURING BILIARY OBSTRUCTION}

Prolonged bile duct ligation leads to periportal fibrosis and ultimately to cirrhosis (120), portal hypertension (120) and portasystemic shunting (121). Based on these findings, prolonged biliary obstruction has been suggested to be a suitable model for cirrhosis in the rat (120). Nonetheless, very little is known about the effects of prolonged biliary obstruction on amino acid and ammonia metabolism in general (122-125) and more specifically interorgan ammonia and glutamine metabolism has never been studied in this situation. Stames et al. (123), using $\alpha-\left[{ }^{14} \mathrm{C}\right]$-aminoisobutyric acid, showed a decrease in amino acid 
uptake in muscle, pancreas and small intestine in rats with obstructive jaundice, whereas liver uptake was unchanged. These authors also observed a decrease in arterial levels of the branched chain amino acid levels (similar to observations during chronic liver failure (126)), whereas all other amino acids, including glutamine, remained unchanged. Prolonged bile duct obstruction induces several other abnormalities (for review, see (122)), including enhanced production of several prostaglandins (127) and immune system dysfunction $(121,128,129)$, endotoxemia and enhanced levels of circulating cytokines $(130,131)$. Although the influence of these factors on ammonia and amino acid metabolism remains to be elucidated, they could be very important, because recently it has been suggested that glutamine metabolism in several organs might be regulated by cytokines $(22,24,132)$. Also, bile salts have been shown to inhibit cerebral oxygen utilization (133).

\section{LIVER FAILURE AND HEPATIC ENCEPHALOPATHY: IMPORTANCE OF GLUTAMINE}

The importance of glutamine during liver failure, portasystemic shunting and hepatic encephalopathy is that glutamine can serve as a non-toxic nitrogen carrier in inter-organ nitrogen exchange $(15,94)$. In addition, it serves as an alternative temporary ammonia detoxification product: definitive ammonia detoxification is only achieved by urea synthesis or urinary ammonia excretion (94). Glutamine serves as a source of new bicarbonate in the kidney (19) and has been suggested to be intimately linked to ammonia coupled proton excretion (6). In addition, its interorgan transport/ exchange are regulated by acid base alterations, which also play a crucial role in hepatic urea synthesis $\left(79,80^{2}\right)$.

\section{AIM OF THIS THESIS}

The aim of this thesis was to study interorgan glutamine and ammonia exchange during various hyperammonemic states induced by liver insufficiency. Specifically, the role of single organs in ammonia and glutamine metabolism was investigated in subsequent studies in one and the same model of liver failure to enable a more accurate comparison of data than usually is possible with literature data. Thus, interorgan glutamine and ammonia exchange were measured during acute and chronic hyperammonemia. Acute hepatic ischemia was used as a model of acute hyperammonemia, portacaval shunting either with or without bile duct ligation was used as a model of chronic hyperammonemia. These models will be briefly discussed in chapter 2 . The studies of interorgan glutamine and ammonia exchange during acute hyperammonemia will be presented in chapters $5,6 \& 7$ of this thesis, whereas the studies during chronic hyperammonemia will be outlined in chapters $8,9,10 \& 11$.

In addition to studying interorgan nitrogen exchange during acute and chronic hyperammonemia, it was the purpose of this work to study the consequences of this interorgan nitrogen exchange on the tissue concentrations of several key metabolites in the synthesis and breakdown of glutamine (and ammonia) and relate these observations to the pathogenesis of hepatic encephalopathy. 
A third aim was to develop a new, good model of chronic hyperammonemia and hepatic encephalopathy, since there appeared to be no such model in the rat. This model is briefly discussed in chapter 2 and more elaborately evaluated in chapters $8,9,10 \& 11$.

Since most of the effects of acute and chronic hyperammonemia are superimposed on a certain degree of (pseudo-) starvation and because malnutrition is very important in patients with liver cirrhosis, the effects of starvation on glutamine and ammonia metabolism were studied. The results of this study are presented in chapter 3 as an introduction to the remaining experimental work.

Because it has recently been suggested that splanchnic glutamine consumption (especially by the intestines) is a major determinant of skeletal muscle glutamine production, the relationship between these organs was studied by eliminating the most important glutamine consumer (gut) from the body (enterectomy model). This seemed worthwhile because intestinal ammonia production is probably the major determinant of systemic ammonia levels and because intestinal ammonia liberation is for a large part derived from intestinal glutamine breakdown. The results are presented in chapter 4.

\section{REFERENCES}

1. Zieve L. Pathogenesis of hepatic encephalopathy. Metab Brain Dis 1987;2:147-165.

2. Meijer AJ, Lamers WH, Chamuleau RAFM. Nitrogen metabolism and ornithine cycle function. Physiol Rev 1990:70:701-748.

3. Newsholme EA, Leech AR. Biochemistry for the Medical sciences. John Wiley \& Sons, New York, 1983.

4. Montgomery R, Dryer RL, Conway TW, Spector AA. Biochemistry. A case-oriented approach. $3^{\text {rd }}$ edition, p. 55, The C.V. Mosby Company, St. Louis, 1980.

5. Cooper AJL, Plum F. Biochemistry and physiology of brain ammonia. Physiol Rev 1987;67:440-519.

6. Halperin ML, Kamel KS, Ethier JH, Stinebaugh BJ, Jungas RL. Biochemistry and Physiology of ammonium excretion. In: The Kidney: Physiology and pathophysiology. Ed: Seldin DW, Giebisch G. Raven Press Ltd, New York, 1992.

7. Duffy TE, Plum F. Hepatic Encephalopathy. In: The liver: Biology and pathobiology. Eds: Arias I, Popper H, Schachter D, Shafritz DA. Raven Press, pp. 693-715, 1982.

8. Good DW, Knepper MA. Ammonia excretion: the role of the renal medulla. Sem Nephrol 1990;10:166-173.

9. Kikeri D, Sun A, Zeidel ML, Heber SC. Cellular $\mathrm{NH}_{4}^{+} / \mathrm{K}^{+}$transport pathways in mouse medullary thick limb of Henle. Regulation by intracellular pH. J Gen Physiol 1992;99:435-461.

10. Lockwood AH, Finn RD, Campbell JA, Richman TB. Factors that affect the uptake of ammonia by the brain: the blood-brain pH gradient. Brain Res 1980;181:259-266.

11. Lin S, Raabe W. Ammonia intoxication: effects on cerebral cortex and spinal cord. J Neurochem 1985;44: 1252-1258.

12. Rudman D, Difulco TJ, Galambos JT, Smith RB, Salam AA, Warten WD. Maximal rates of excretion and synthesis of urea in normal and cirrhotic subjects. J Clin Invest 1973;52:2241-2249.

13*. Lockwood AH, McDonald JM, Reiman RE, Gelbard AS, Laughlin JS, Duffy TE, Plum F. The dynamics of ammonia metabolism in man. Effects of liver disease and hyperammonemia. J Clin Invest 1979;63:449-460.

13. Duda GD, Handler P. Kinetics of ammonia metabolism in vivo. J Biol Chem 1958;232:303-314.

14. Lacey JM, Wilmore DW. Is glutamine a conditionally essential amino acid? Nutr Rev 1990;48:297-309.

15. Souba WW, Smith RJ, Wilmore DW. Glutamine metabolism by the intestinal tract. JPEN 1985;9:608-617.

16. Windmueller HG. Glutamine utilization by the small intestine. Adv Enzyme 1982;53:210-237.

17. Windmueller HG, Spaeth AE. Uptake and metabolism of plasma glutamine by the small intestine. J Biol Chem 1974;249:5070-5079. 
18. Souba WW. Interorgan ammonia metabolism in health and discase: A surgeon's view. JPEN 1987;11:569-579.

19. Halperin ML, Ethier JH, Kamel KS. The excretion of ammonium ions and acid base balance. Clin Biochem 1990;23:185-188.

20. Souba WW, Klimberg VS, Plumley DA, Salloum RM, Flynn TC, Bland KI, Copeland EM. The role of glutamine in maintaining a healthy gut and supporting the metabolic response to injury and infection. $J$ Surg Res 1990;48:383-391.

21. Rennie MJ, Hundal HS, Babij P, MacLennan P, Taylor PM, Watt PW. Characteristics of a glutamine carrier in skeletal muscle have important consequences for nitrogen loss in injury, infection, and chronic disease. Lancet 1986;ii:1008-1011.

22. Robinson $A B$, Robinson LR. Distribution of glutamine and asparagine residues and their near neighbors in peptides and proteins. Proc Natl Acad Sci USA 1991;88:8880-8884.

23. Souba WW, Herskowotz K, Klimberg VS, Salloum RM, Plumley DA, Flynn TC, Copeland EM. The effects of sepsis and endotoxemia on gut glutamine metabolism. Ann Surg 1990;211:543-551.

24. Austgen TR, Chen MK, Dudrick PS, Copeland EM, Souba WW. Cytokine regulation of intestinal glutamine utilization. Am J Surgery 1992;163:174-175.

25. Wilmore DW, Smith RJ, O'Dwyer ST, Jacobs DO, Ziegler TR, Wang XD. The gut: A central organ after surgical stress. Surgery 1988; 104:917-923.

26. Ardawi MSM, Newsholme EA. Glutamine metabolism in lymphocytes of the rat. Bioch $J$ $1983 ; 212: 835-842$.

27. O'Dwyer ST, Smith RJ, Hwang TL, Wilmore DW. Maintenance of small bowel mucosa with glutamine-enriched parenteral nutrition. JPEN 1989;13:579-585.

28. Jacobs DO, Evans DA, Mealy K, O'Dwyer ST, Smith RJ, Wilmore DW. Combined effects of glutamine and epidermal growth factor on the rat intestine. Surgery 1988;104:358-364.

29. Klimberg VS, Salloum RM, Kasper M, Plumley DA, Dolson DJ, Hautamaki WR, Mendenhall WR, Bova FC, Bland KI, Copeland EM, Souba WW. Oral glutamine accelerates healing of the small intestine and improves outcome after whole abdominal radiation. Arch Surg 1990;125: 1040-1045.

30. Wagenmakers AJM. Branched chain amino acid degradation in muscle. Thesis, Nijmegen, The Netherlands, 1984.

$30^{\circ}$ Brainard JR, Kyner E, Rosenberg GA. ${ }^{13} \mathrm{C}$ Nuclear magnetic resonance evidence for $\gamma$-aminobutyric acid formation via pyruvate carboxylase in rat brain: a metabolic basis for compartmentation. $J$ Neurochem 1989;53:1285-1292.

31. Lund P. A radiochemical assay for glutamine synthetase, and activity of the enzyme in rat tissues. Bioch J 1970;1 18:35-39.

32. Windmueller HG, Spaeth AE. Intestinal metabolism of glutamine and glutamate from the lumen as compared to glutamine from blood. Arch Bioch Bioph 1975,171:662-672.

33. Windmueller HG, Spaeth AE. Identification of ketone bodies and glutamine as the major respiratory fuels in vivo for postabsorptive rat small intestine. J Biol Chem 1978;253:69-76.

34. Steiner M, Bourges HR, Freedman LS, Gray SJ. Effect of starvation on the tissue composition of the small intestine in the rat. Am J Physiol 1968;215:75-77.

35. Weber FL, Veach GL. The importance of the small intestine in gut ammonium production in the fasting dog. Gastroenterology 1979;77:235-240.

36. Weber FL, Friedman DW, Fresard KM. Ammonia production from intraluminal amino acids in canine jejunum. Am J Physiol 1988;254:G264-G268.

37. Chang TW, Goldberg AL. The metabolic fates of amino acids and the formation of glutamine in skeletal muscle. J Biol Chem 1978;253:3685-3695.

38. Imler M, Schlienger JL. The effect of chronic uremia on portal and systemic ammonemia in normal and portal-strictured rats. J Lab Clin Med 1979;94:872-878.

39. Wahren J. Extra-hepatic ammonia metabolism, in: Advances in ammonia metabolism and hepatic encephalopathy. (Soeters PB, Wilson JHP, Meijer AJ, Holm E, eds.) Excerpta Medica, Amsterdam, pp. 121-129, 1988 .

40. Ganda OP, Ruderman NB. Muscle nitrogen metabolism in chronic hepatic insufficiency. Metabolism 1976;25:427-435.

41. Ardawi MSM. Skeletal muscle glutamine production in thermally injured rats. Clin Sci 1988;74:165-172.

42. Smith RJ, Larson S, Stred SE, Durschlag RP. Regulation of glutamine synthetase and glutaminase activities in cultured skeletal muscle cells. J Cell Physiol 1984;120:197-203.

43. Nelson D, Rumsey WL, Erecinska M. Glutamine catabolism by hear muscle. Biochem J 1992;282:559-564. 
44. Kelso TS, Shear CR, Max SR. Enzymes of glutamine metabolism in inflammation associated with skeletal muscle hypertiophy. Am J Physiol 1989;257:E885-E894.

45. Eriksson LS, Broberg S, Bjorkman O, Wahren J. Ammonia metabolism during exercise in man. Clin Physiol 1985;5:325-336.

46. Chabrier G, Schlienger JL, Imler M. Etude du métabolisme musculaire de l'ammoniaque sur le train postérieur du rat intact. CR Soc Biol 1982;176:716-722.

47. Rosado A, Flores G, Mora J, Soberon G. Distribution of an ammonia load in the normal rat. Am J Physiol 1962:203:37-42.

48. Warter JM, Marescaux Ch, Brandt Ch, Rumbach L, Micheletti G, Chabrier G, Imler M, Kurtz D. Sodium valproate associated with phenobarbital: effects on ammonia metabolism in humans. Epilepsia 1983;24:628-633.

49. Warter JM, Brandt Ch, Marescaux Ch, Rumbach L, Micheletti G, Chabrier G, Krieger J, Imler M. The renal origin of sodium valproate-induced hyperammonemia in fasting humans. Neurology 1983;33:1136-1140.

50. Hills AG, Reid EL, Kerr WD. Circulatory transport of L-glutamine in fasted mammals: cellular sources of urine ammonia. Am J Physiol 1967;223:1470-1476.

51. Fine A. The effects of ammonia infusion on ammonia and glutamine metabolism by liver and muscle in the normal dog. Contrib Nephrol 1985;47:1-8.

52. Gjedde A, Lockwood AH. Duffy TE, Plum F. Cerebral blood flow and metabolism in chronically hyperammonemic rats: Effect of an acute ammonia challenge. Ann Neurol 1978;3:325-330.

53. Ruderman NB, Lund P. Amino acid metabolism in skeletal muscle. Regulation of glutamine and alanine release in the perfused rat hindquarter. Israel J Med Sci 1972;8:295-302.

54. Deutz NEP, Dejong CHC, Reijven PLM, Soeters PB. In vivo ammonia and glutamine flux measurements during hyperammonemia in rats and pigs, in: Progress in hepatic encephalopathy and metabolic nitrogen exchange. (Bengtsson F, Jeppsson B, Almdal T, Vilstrup H, eds.) CRC Press, Boca Riton, pp. 329-339, 1991.

55. Schröck H, Cha CM, Goldstein L. Glutamine release from hindlimb and uptake by kidney in the acutely acidotic rat. Biochem J 1980; 188:557-560.

56. Goldberg AL, Chang TW. Regulation and significance of amino acid metabolism in skeletal muscle. Fed Proc 1978;37:2301-2307.

57. Lowell BB, Ruderman NB, Goodman MN. Regulation of myofibrillar protein degradation in rat skeletal muscle during brief and prolonged starvation. Metabolism 1986;35:1121-1127.

58. Ruderman NB, Berger M. The formation of glutamine and alanine in skeletal muscle. J Biol Chem 1974;249:5500-5506.

59. Kominz DR, Hough A, Symonds P, Laki K. The amino acid composition of actin, myosin, tropomyosin and the meromyosins. Arch Bioch Biophys 1954;50:148-159.

60. Norenberg MD, Martinez-Hemandez A. Fine structural localization of glutamine synthetase in astrocytes of rat brain. Brain Res 1979;161:303-310.

61. Bradford HF, Ward HK, Thomas AJ. Glutamine - A major substrate for nerve endings. J Neurochem 1978:30:1453-1459.

62. Benjamin AM. Control of glutaminase activity in rat brain cortex in vitro: influence of glutamate, phosphate, ammonium, calcium and hydrogen ions. Brain Res 1981;208:363-377.

63. Kvamme E, Lenda K. Regulation of glutaminase by exogenous glutamate, ammonia and 2oxoglutarate in synaptosomal enriched preparation from rat brain. Neurochem Res 1982;7:667-678.

64. Martinez-Hernandez A. Bell KP, Norenberg MD. Glutamine synthetase: Glial localization in brain. Science 1977; 195:1356-1358.

65. Webster LT, Gabuzda GJ. Ammonium uptake by the extremities and brain in hepatic coma. J Clin Invest 1958:37:414-424.

66. Welboume TC, Phromphetcharat V, Givens G, Joshi S. Regulation of interorgan glutamine flow in metabolic acidosis. Am J Physiol 1986;250:EA57-EA63.

67. Vinay P, Allignet E, Pichette C, Watford M, Lémieux G, Gougoux A. Changes in renal metabolite profile and ammoniagenesis during acute and chronic metabolic acidosis in dog and rat. Kidney Int 1980;17:312-325.

68. Schröck H, Goldstein L. Interorgan relationships for glutamine metabolism in normal and acidotic rats. Am J Physiol 1981;240:E519-E525.

69. Phromphetcharat V. Welboume TC. Renal glutamine extraction and gut/liver interaction in glutamine homeostasis. Contrib Nephrol 1985;47:9-14.

70. Welbourne TC. Effect of metabolic acidosis on hindquarter glutamine and alanine release. Metabolism 1986:35:614-618. 
71. Welboume TC, Childress D, Givens G. Renal regulation of interorgan glutamine flow in metabolic acidosis. Am J Physiol 1986;251:R858-R866.

72. Welboume TC. Hepatic glutaminase flux regulation of glutamine homeostasis. Studies in vivo. Biol Chem Hoppe Seyler 1986;367:301-305.

73. Welbourne TC, Dass PD. Gamma glutamyltransferase contribution to renal ammoniagenesis in vivo. Pflügers Arch 1988;411:573-578.

74. Warter JM, Imler M, Marescaux C, Chabrier G, Rumbach L, Micheletti G, Krieger J. Sodium valproate-induced hyperammonemia in the rat: role of the kidney. Eur J Pharmacol 1983;87:177-182.

75. Imler M, Chabrier G, Marescaux C, Warter JM. Effects of 2,4-dinitrophenol on renal ammoniagenesis in the rat. Eur J Pharmacol 1986;123:175-179.

76. Warter JM, Marescaux C, Chabrier G, Rumbach L, Micheletti B, Imler M. Métabolisme rénal de la glutamine chez l'homme au cours des traitements par le valproate de sodium. Rev Neurol (Paris) 1984;140:370-371.

77. Silbernagl S. The renal handling of amino acids and oligopeptides. Physiol Rev 1988;68:91 1-1007.

$78^{\mathrm{a}}$. Cooper AL, Filc-DeRicco S, Gelbard AS. L ${ }^{13} \mathrm{~N}$-glutamate metabolism in normal rat kidney, in: Progress in hepatic encephalopathy and metabolic nitrogen exchange. (Bengtsson F, Jeppsson B, Almdal T, Vilstrup H, eds.) CRC Press, Boca Raton, pp. 341-351, 1991.

78. Atkinson DE, Bourke E. The role of ureagenesis in pH homeostasis. TIBS 1984;9:297-300.

79. Haüssinger D, Kaiser S, Stehle T, Gerok W. Structural and functional organization of hepatic ammonia metabolism: pathophysiological consequences, in: Advances in ammonia metabolism and hepatic encephalopathy. (Soeters PB, Wilson JHP, Meijer AJ, Holm E, eds.) Excerpta Medica, Amsterdam, pp. 26-36, 1988.

$80^{2}$. Kaiser S, Gerok W, Haüssinger D. Ammonia and glutamine metabolism in human liver slices: new aspects on the pathogenesis of hyperammonemia in chronic liver disease. Eur J Clin lnvest $1988 ; 18: 535-542$.

$80^{\mathrm{b}}$. Verhoeven AJ, van Iwaarden JF, Joseph SK, Meijer AJ. Control of rat-liver glutaminase by ammonia and $\mathrm{pH}$. Eur J Biochem 1983; 133:24 1-244.

81. Welbourne TC. Role of the lung in glutamine homeostasis. Contrib Nephrol 1988;63:178-182.

82. Plumley DA, Austgen TR, Salloum RM, Souba WW. Role of the lungs in maintaining amino acid homeostasis. JPEN 1990; 14:569-573.

83. Austgen TR, Plumley DA, Souba WW. Simple method of determining pulmonary blood flow in the anesthetized rat. J Invest Surg 1991;4:81-86.

84. Plumley DA, Souba WW, Hautamaki D, Martin TD, Flynn TC, Rout R, Coperland EM. Accelerated lung amino acid release in hyperdynamic septic surgical patients. Arch Surg 1990;125:57-61.

85. Coulson RA, Hemandez T. Site of synthesis of amino acids in the intact cayman. Am J Physiol 1967;213:411-417.

86. Krames BB, van Liere EJ. The hear weight and ventricular weights of normal adult albino rats. Anat Rec 1975; 156:461-464.

87. Fan FC, Chen RYZ, Schuessler GB, Chien S. Effects of hematocrit variations on regional hemodynamics and oxygen transport in the dog. Am J Physiol 1980;238:H545-H552.

88. Sasaki Y, Wagner HN. Measurement of the distribution of cardiac output in unesthetized rats. J Appl Physiol 1971;30:879-884.

89. Deutz NEP, Reijven PLM, Athanasas G, Soeters PB. Postoperative changes in hepatic, intestinal, splenic and muscle amino acid and ammonia fluxes in pigs. Clin Sci 1992: in press.

90. Steele RD. Hyperammonemia and orotic aciduria in portacaval shunted rats. J Nutr 1984;114:210-216.

91. Record CO. Neurochemistry of hepatic encephalopathy. Gut 1991;32:1261-1263.

92. Sherlock S. Diseases of the liver and biliary system. Blackwell Sci Publ Oxford, 8th Edition, 1989.

93. Ganz R, Swain M, Traber P, DalCanto M, Butterworth RF, Blei AT. Ammonia induced swelling of rat cerebral cortex slices: implications for the pathogenesis of brain edema in acute hepatic failure. Metab Brain Dis 1989;4:213-223.

94. Conn HO, Bircher J. Hepatic encephalopathy: management with lactulose and related carbohydrates. Medi-Ed Press, East Lansing, Michigan, USA, 1988.

95. Tyor MP, Owen EE, Berry JN, Flanagan JF. The relative role of extremity, liver and kidney as ammonia receivers and donors in patients with liver disease. Gastroenterology 1960;39:420-424.

96. Berry JN, Flanagan JF, Owen EE, Tyor MP. The kidney as a source of blood ammonia in resting and hyperventilated cirrhotics. Clin Res 1959;7:154-155.

97. Imler M, Schlienger J-L, Chabrier G, Simon C. Arterial ammonemia changes of renal origin induced in the rat by acid and alkaline diets. Res Exp Med 1986;186:353-363. 
98. Bessman SP, Bradley JE. Uptake of ammonia by muscle. Its implications in ammoniagenic coma. New Eng J Med 1985;253:1 143-1147.

99. Cooper AJL, McDonald JM, Gelbard AS, Gledhill RF, Duffy TE. The metabolic fate of ${ }^{13} \mathrm{~N}$-labeled ammonia in rat brain. J Biol Chem 1979;254:4982-4992.

100. Cooper AJL, Mora SN, Cruz NF, Gelbard AS. Cerebral ammonia metabolism in hyperammonemic rats. J Neurochem 1985;44:1716-1723.

101. Hawkins RA, Miller AL, Nielsen RC, Veech RL. The acute action of ammonia on rat brain metabolism in vivo. Biochem J 1973;134:1001-1008.

102. Hindfelt B, Plum F, Duffy TE. Effect of acute ammonia intoxication on cerebral metabolism in rats with portacaval shunts. J Clin Invest 1977;59:386-396.

103. Watanabe A, Shiota T, Takei N, Fujiwara M, Nagashima H. Ammonia detoxification by accelerated oxidation of branched chain amino acids in brains of acute hepatic failure rats. Biochem Med Metab Biol 1986;35:367-375.

104. Bosman DK, Deutz NEP, de Graaf AA, van der Hulst RWN, van Eijk HMH, Bovée WMMJ, Maas MAW, Jöming GGA, Chamuleau RAFM. Changes in brain metabolism during hyperammonemia and acute liver failure: Results of a comparative 'H-NMR spectroscopy and biochemical investigation. Hepatology 1990;12:281-290.

105. Peterson C, Giguère JF, Cotman CW, Butterworth RF. Selective loss of N-Methyl-D-Asparate sensitive $\mathrm{L}-\left[{ }^{3} \mathrm{H}\right]$ Glutamate binding sites in rat brain following portacaval anastomosis. J Neurochem 1990;55:386-390.

106. Butterworth RF, Girard G. Giguere JF. Regional differences in the capacity for ammonia removal by brain following portocaval anastomosis. J Neurochem 1988;51:486-490.

107. Deutz NEP. Pathophysiological aspects of acute hepatic encephalopathy in the rat. An electroencephalographic, biochemical and in vivo brain NMR spectroscopic study. Thesis, Amsterdam, The Netherlands, 1988.

108. Deutz NEP, Chamuleau RAFM, de Graaf AA, Bovée WMM, de Beer R. In vivo ${ }^{31} \mathrm{P}$ NMR spectroscopy of the rat cerebral contex during acute hepatic encephalopathy. NMR Biomed 1988;1:101-106.

109. Basile AS, Jones EA, Skolnick P. The pathogenesis and treatment of hepatic encephalopathy: evidence for the involvement of benzodiazepine receptor ligands. Pharm Rev 1991;43:27-71.

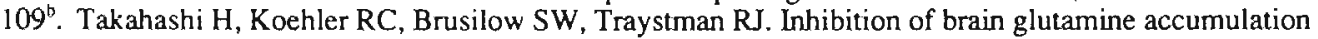
prevents cerebral edema in hyperammonemic rats. Am J Physiol 1991;261:H825-H829.

110. Tannen RL. Ammonia metabolism. Am J Physiol 1978;235:F265-277.

111. Yablon SB, Relnan AS. Glutamine as a regulator of renal ammonia production in the rat. Kidney Int 1977;12:546 (abstract).

112. Owen EE. Johnson JH, Tyor MP. The effect of induced hyperammonemia on renal ammonia metabolism. J Clin Invest 1961;40:215-221.

113. Owen EE, Robinson RR. Amino acid extraction and ammonia metabolsim by the human kidney during the prolonged administration of ammonium chloride. J Clin Invest 1963;42;263-276.

114. Welbourne T, Weber M, Bank N. The effect of glutamine administration on urinary ammonium excretion in normal subjects and patients with renal disease. J Clin Invest 1972;51:1852-1860.

115. Owen EE, Tyor MP, Flanagan JF. Berry JN. The kidney as a source of blood ammonia in patients with liver disease: the effect of acetazolamide. J Clin Invest 1960;39:288-294.

116. Imler M, Schlienger J-L, Chabrier G, Comte F. Origine rénale de l'hyperammoniémie provoquée par un régime hyperprotidique chez le rat normal ou porteur d'une stricture portale. Gastroenterol Clin Biol 1983;7:740-745.

117. Gibson GE, Zimber A, Krook L, Visek WJ. Nucleic acids and brain and intestinal lesions in ammonia intoxicated mice. Fed Proc 1970;30:578A (abstract).

118. Zimber A, Visek WJ. Effect of urease injections on DNA synthesis in mice. Am J Physiol 1972;223:1004-1008.

119. Dang HC, Visek WJ. Some characteristics of blood in nornal and immune rabbits after urease injection. Am J Physiol 1968;215:502-505.

120. Kountouras J, Billing BH, Scheuer PJ. Prolonged bile duct obstruction: a new experimental model for cirrhosis in the rat. Br J Exp Path 1964;65:305-311.

121. Dunn CW, Horton JW, Megison SM, Vuitch MF. Contribution of portal systemic shunt to Kuppfer cell dysfunction in obstructive jaundice. J Surg Res 1991;50:234-239.

122. O'Connor MJ. Mechanical biliary obstruction. Amer Surg 1985;51:245-251.

123. Stames HF, Conti PS, Warren RS, Jeevanandam M, Brennan MF. Altered peripheral amino acid uptake in obstructive jaundice. J Surg Res 1987;42:383-393. 
124. Walshe JM. Disturbances of amino acid metabolism following liver injury. A study by means of paper chromatography. Quart J Med 1953:22:483-505.

125. Maddison JE, Dodd PR, Morrison M, Johnston GAR, Farrell GC. Plasma GABA, GABA-like activity and the brain GABA-benzodiazepine receptor complex in rats with chronic hepatic encephalopathy. Hepatology 1987;7:621-628.

126. Benjamin IS, Engelbrecht GHC, Saunders SJ, van Hoom Hickman R. Amino acid imbalance following portal diversion in the rat. The relevance of nutrition and of hepatic function. $J$ Hepatology 1988;7:208-214.

127. O'Neill PA, Wait RB, Kahng KU. Role of renal sympathetic nerve activity in renal failure associated with obstructive jaundice in the rat. Am J Surg 1991;161:662-667.

128. Greve JWM, Gouma DJ, Soeters PB, Buurman WA. Suppression of cellular immunity in obstructive jaundice is caused by endotoxins. A study in germ-free rats. Gastroenterology 1990;98:478-485.

129. Roughneen PT, Gouma DJ, Kulkarni AD, Fanslow WF, Rowlands BJ. Impaired specific cellmediated immunity in experimental biliary obstruction and its reversibility by intemal biliary drainage. J Surg Res 1986;41:113-125.

130. Greve JWM. Complications in obstructive jaundice. An experimental study on etiology and prevention. Thesis, Maastricht, The Netherlands, 1990.

131. Bemelmans MHA, Greve JW, Gouma DJ, Buurman WA. Cytokines tumor necrosis factor and interleukin-6 in experimental biliary obstruction in mice. Hepatology 1992:15:1 132-1136.

132. Austgen TR, Chen MK, Salloum RM, Souba WW. Glutamine metabolism by the endotoxin-injured lung. J Trauma 1991;31:1068-1075.

133. Lascelles PT. Oxygen electrode studies on rat respiration in hepatic coma. Exp Biol Med 1971;4:104-106. 



\section{Chapter 2}

\section{Models}

\section{INTRODUCTION}

In this chapter, the animal models used in the experimental work in this thesis will be defined. Furthermore, the technical aspects and theoretical backgrounds of blood flow measurements across several organs using the para-aminohippuric acid indicator dilution method will be addressed.

\section{MODEL OF ACUTE LIVER INSUFFICIENCY}

Clinical background Acute liver failure and the resulting hyperammonemia predominantly occur in patients with underlying chronic liver disease (1). In these patients, sudden elevations of plasma ammonia levels are precipitated by exogenous or endogenous protein loads as for example after gastrointestinal hemorrhage (1). Acute severe hyperammonemia not superimposed on chronic liver failure is a condition not so commonly encountered in clinical practice. Mostly it results from fulminant hepatic failure in patients with hepatitis, paracetamol overdose or rejection of a transplanted liver $(1,2)$.

Existing models Suitable experimental animal models of hyperammonemia induced by acute liver failure should meet certain requirements. Such models should ideally not only display (the effects of) hyperammonemia, but also several other effects of impaired liver function, such as e.g. altered amino acid metabolism, diminished urea synthesis capacity and impaired gluconeogenesis (for review, see (1-4)). Furthermore, these models should be reproducible. Portasystemic shunting is not a prerequisite of these models, but, if present, it does bear some resemblance with clinical practice, since some degree of intra- or extrahepatic shunting of portal venous blood very often occurs during acute liver failure.

Several animal models have been utilized in the literature to study the effects of acute severe hyperammonemia (in rats, unless stated otherwise): hepatectomy in dogs (5) or rats $(6,7)$, partial hepatectomy (8), partial hepatectomy after $\mathrm{CCl}_{4}$ administration (9), portacaval shunting after $\mathrm{CCl}_{4}$ administration (10), galactosamine-induced acute liver failure in rabbits (11), thioacetamide-induced liver failure (12), acute ammonia administration to normal rats $(13,14)$ or cats $(15)$ or to portacaval shunted rats $(16-18)$, urease administration to normal 
rats (19), normal mice $(20,21)$ or portacaval shunted rats (22) and acute liver ischemia induction (8,19,23-30). Some of these models only represent the effects of acute ammonia loading (urease injections and ammonia administration to normal rats). Others, like the hepatectomy models, require several surgical interventions and extensive diversion of blood flow of several infra-diaphragmatic organs $(6,7)$. In addition, the latter model shows a wide diversity in time span between the various surgical interventions, which makes comparison of results very difficult, if not impossible. The galactosamine $(11,31,32)$ and thioacetamide models (12) suffer from the drawback that the symptoms of hepatitis are very pronounced. Also, the doses of galactosamine and thioacetamide administered in different laboratories show considerable variation. This precludes adequate comparison of literature data. In addition, the galactosamine model does not appear to be applicable in rats, because of batch variations in susceptibility to galactosamine and in the development of hepatic encephalopathy (32). In addition, this model is expensive in rabbits (32).

Choice of the model For the experimental work presented in this thesis during acute liver insufficiency, the acute liver ischemia model in the rat was chosen. In this model interorgan nitrogen exchange across intestines, muscle, brain and kidney was studied. The rat was chosen because this animal is small, easy to handle, cheap and easy to obtain. The acute liver ischemia model was chosen because it has been shown to be very reproducible in several laboratories $(8,19,23-30)$. Acute liver ischemia is induced by ligating the hepatic artery one day after the construction of a portacaval shunt (19). The portacaval shunt is constructed either by the suture technique as described by Lee and Fisher (33), or by the button technique described by Funovics (34). Ligation of the hepatic artery is accomplished either with $(24,35)$ or without $(8,19,23,29,30)$ relaparotomy (mostly) one day after portacaval shunting. In the present thesis, the button technique was applied using polyethylene buttons (I.D. 0.17 $\mathrm{cm}$, O.D. $0.23 \mathrm{~cm}$ ) and hepatic artery ligation was always performed by relaparotomy, the day after portacaval shunt construction (except in chapter 6: two days after portacaval shunting).

Portacaval shunting by the button technique is a rapid procedure, resulting in portalsystemic shunts with standardized diameters (34). Operation time is approximately $15-20$ min, portal vein clamping time less than $5 \mathrm{~min}$ and inferior caval vein clamping time is less than $1 \mathrm{~min}$ (34) (see chapter 5). Survival rates in the studies reported in this thesis were better than $90 \%$.

Ligation of the hepatic artery, the day after portacaval shunting is facilitated by leaving a silk wire around the hepatic artery during portacaval shunting (chapters 5, $6 \& 7$ ). The total procedure takes only approximately $8 \mathrm{~min}$ and has no mortality.

Although the induction of acute liver ischemia bears the disadvantage of a necrotic organ in the abdomen, it has the advantage of excellent reproducibility $(8,19,23-30)$ and combines the effects of severe hyperammonemia, portacaval shunting and impaired liver function in one model. Rats with acute liver ischemia develop progressive hyperammonemia, hypoglycemia and hypothermia $(8,19,23,35)$. Progressive hepatic encephalopathy develops, resulting in coma within 8 hours after liver ischemia induction (8), and finally a 100\% mortality within 10-12 h. Acute liver ischemia induction has repeatedly been used as a reproducible rat model of acute severe hyperammonemia and hepatic encephalopathy $(6,8,19,23-28)$, identical by clinical, EEG and visual evoked response criteria to the hepatic encephalopathy of other models of acute severe hyperammonemia $(8,19)$. The experimental work performed in this model will be presented in chapters 5,6 and 7 . 


\section{MODEL OF CHRONIC LIVER INSUFFICIENCY}

Clinical background Especially in the rat, multiple models have been developed to study the pathophysiology of chronic liver disease (10,33,34,36-39), but none of them appears to be entirely suitable (39). In their recent review, Mullen et al. considered several of the problems related to the use of rat models of chronic liver failure (39).

In essence, the failure to develop a good model of chronic liver failure, despite an enormous amount of research, results from the complexity and variability of chronic liver failure in humans (1). Thus, chronic liver failure in humans is mostly the consequence of cirrhosis of the liver, but can also be due to e.g. metastatic malignant invasion or urea cycle deficiencies (1). Cirrhosis of the liver is generally accompanied by portasystemic shunting, either intra- or extrahepatic $(4,40)$, but portacaval shunting is not always the consequence of liver cirrhosis, as it can also be caused e.g. by portal vein thrombosis (1). In addition, chronic liver failure is often accompanied by some degree of hepatic encephalopathy $(1,39)$. Approximately $60-70 \%$ of patients with cirrhosis only suffer from subclinical hepatic encephalopathy $(1,39)$, although manifest encephalopathy is intermittently precipitated in these patients by various provoking factors, leading to a characteristic fluctuating course (1). Thus, any animal model of chronic liver failure should ideally enable the study of all these components, as well as the various causes of liver failure. Most animal models, however, meet only one or two of these criteria and unfortunately suffer from bad reproducibility (39). Consequently, a satisfactory animal model of chronic liver disease with hepatic encephalopathy and abnormalities in nitrogen metabolism, as is present in man (39), is still lacking.

The proposed causal relation between hyperammonemia and the pathogenesis of hepatic encephalopathy $(1,4,41)$ has led to the additional requirement of hyperammonemia in adequate models of chronic liver failure. It is questionable whether a good animal model of chronic liver failure must have high ammonia levels and whether higher ammonia levels would be preferable, since most cirrhotic patients do not have continuous hyperammonemia (1). Mostly, episodes of hyperammonemia and/or hepatic encephalopathy are precipitated by protein overloads, gastrointestinal bleeding, infections, electrolyte imbalances, constipation or alcohol withdrawal (1). To date, the ideal animal model of chronic liver failure is lacking and probably the model used in a specific study should depend on the specific question that is addressed in that study and on the presumed pathogenetic mechanism.

Existing models Several experimental animal models have been utilized to study the effects of chronic liver failure $(10,33,34,36-39)$. In view of their potential reproducibility, two rat models do seem appropriate to study chronic liver failure in the rat: carbon-tetrachloride $\left(\mathrm{CCl}_{4}\right)$ cirrhosis and end-to-side portacaval shunting (39).

The $\mathrm{CCl}_{4}$-induced liver failure model (reviewed in (39)) gives rise to extensive cirrhosis of the liver. The doses of $\mathrm{CCl}_{4}$ given in various research institutes and the response of individual animals to them may vary considerably, which adversely affects reproducibility (37). The model has the disadvantage of the inflammatory response in the liver and the high mortality (37). Portal hypertension, portasystemic shunting, ascites and moderate hyperammonemia are commonly observed in this model, but urea cycle enzyme activity is not depressed ((39) and references quoted therein). The frequently encountered ascites in the $\mathrm{CCl}_{4}$ model presents the investigator with difficulties in calculating enzyme kinetics, and results on urea cycle activity obtained in this rat model should be interpreted cautiously. 
Symptoms of hepatic encephalopathy have been reported to vary considerably depending on the protocol of induction of cirrhosis ((39) and references quoted therein).

Portacaval shunting has the advantage of good reproducibility in case a real portacaval anastomosis is surgically constructed end-to-side (39). Lee and Fisher (33) described the traditional portacaval shunting by the suture technique in 1961. Funovics (34) adapted and modified this technique and reported the button technique of portacaval shunting in 1975. The latter technique was also adapted for application in dogs (42), to provide a substitute for the naturally occurring spontaneously portacaval shunted dog (43), since the latter is difficult to obtain and expensive. Other authors used different techniques of inducing portasystemic shunting, as e.g. the construction of a portal stricture $(44,45)$ or subcutaneous transposition of the spleen (36). These latter two techniques probably suffer from poor reproducibility, since the location and extent of hepatofugal shunts is unpredictable. In general, portacaval shunting techniques induce, apart from portacaval shunting, also liver atrophy (38) and failure $(17,46)$, testicular atrophy $(38)$, hyperammonemia $(38,46,47)$, anorexia (38) and subtle signs of encephalopathy $(17,39,48)$, but no cirrhosis of the liver or ascites. The portacaval shunted rat probably is a good model to study subclinical hepatic encephalopathy in the rat (39).

Portacaval shunting has been extensively studied as a model of chronic liver failure $(10,17,33,34,38,39,42,46,47,49,50)$. Portacaval shunting leads to consistent results if used repeatedly in the same laboratory, but unfortunately leads to inconsistency of results between different laboratories, especially concerning postoperative weight gain (39). Several of these discrepancies might be attributable to differences in surgical technique $(33,34)$, preoperative preparation $(10)$, time span between shunt construction and experimental observation, diet and age and weight of the animals used (39). In the immediate postsurgery phase, rapid weight loss occurs during 5-10 days $(17,47,49)$ of approximately $10 \%$ (49) to $25 \%$ (38) of initial body weight depending on age of the rats and whether the suture (33) or button technique (34) of shunting are used (49). Systemic ammonia levels are acutely elevated $(17,47)$. The weight loss is reflected by a more than $50 \%$ reduction in liver weight $(17,47)$ as well as testis weight reduction $(38)$. After $2(17,47)$ to 4 weeks $(38)$, rats start to regain weight again and this is generally reflected by a decrease in plasma ammonia levels $(38)$, both of which have been related to the formation of hepatopetal shunts $(38,49)$. These observations stress the importance of studying these rats before such shunts develop (49), i.e. before the portacaval shunted rats start to regain weight.

The lacking cirrhosis in portacaval shunting models and the only moderate degree of encephalopathic changes and hyperammonemia, has led several investigators to develop new models, combining liver cirrhosis with portacaval shunting (10,36,37). Thus, Hanisch et al. (10) reported a model of $\mathrm{CCl}_{4}$-induced cirrhosis followed by portacaval shunting as described by Lee and Fisher (33). This model has an extremely high mortality, unless a specific preoperative regimen is applied (10). Moreover, the model involves 16 weeks of repeated $\mathrm{CCl}_{4}$ administration under ether anesthesia before the shunt is constructed (10).

Bile duct ligation in the rat has been used to simulate primary biliary cirrhosis in humans (37), a cause of portasystemic shunting, hyperammonemia and hepatic encephalopathy (1). Common bile duct ligation in the rat is very reproducible in causing jaundice and, therefore, several aspects related to cholestatic jaundice (for review, see (51)). These aspects probably also play a role in at least a limited number of patients with chronic liver insufficiency not caused by biliary cirrhosis, since jaundice often is present in that group, too (1). There has 
been some discussion whether prolonged biliary obstruction in the rat leads to cirrhosis ( $(37)$ and references quoted therein). Kountouras et al. (37) found that biliary obstruction for less than 14 days merely causes fibrosis, whereas thereafter cirshosis ensues. Prolonged bile duct ligation has been shown to induce portal hypertension (37) and portasystemic shunting (52), as well as immune system dysfunction (52-55). Bile duct ligation induces some of the abnormalities in amino acid metabolism and glucose and lactate metabolism also encountered during chronic liver disease in humans (56) and surely induces parenchymal damage to the liver (37).

Portacaval shunting combined with common bile duct ligation has recently been suggested to be a reliable, new model for chronic hyperammonemia (36). In this model subcutaneous transposition of the spleen was followed 3 weeks later by ligation of the portal vein (36). 5 Weeks after portal vein ligation the common bile duct was ligated and experiments were performed 1 week after bile duct ligation (36). This model was stated to induce a more pronounced clinical degree of hepatic encephalopathy (36). Theoretically, this model combines the effects of portasystemic shunting with the occurrence of (biliary) cirrhosis of the liver and could lead to a more pronounced degree of liver failure. This might more profoundly reduce urea synthesis capacity, resulting in an additional elevation of systemic ammonia levels. Furthermore, the bile duct ligation and ensuing bilirubinemia simulate, although to an exaggerated extent and by different mechanisms, the impaired bile excretion in patients with chronic cirrhosis of the liver. The presence of bile in the gut lumen has been shown to be essential in reducing endotoxin transmucosal translocation and to modulate cellular immunity $(54,55)$. The relationship between impaired immunity in cirrhotics (1) and impaired bile excretion is highly speculative, but attractive and a model enabling the study of some aspects of this putative relationship seems worthwhile.

Thus, we adopted and modified the portacaval shunted/biliary obstructed model originally described by Maddison (see above) (36) for studying nitrogen metabolism during chronic liver failure in the rat. Pilot studies in our lab showed that ammonia levels were comparably elevated in portacaval shunted/biliary obstructed rats, in contrast with the findings in the original model described above, whereas the clinical picture suggested more profound hepatic encephalopathy as had been shown previously (36). In addition, amino acid patterns were remarkably different, suggesting that the model could provide valuable information concerning the pathophysiology of chronic encephalopathy.

We therefore chose this model of portacaval shunting and bile duct ligation to study interorgan glutamine and ammonia exchange during chronic liver failure. To improve the reproducibility of the model, we modified it to the extent that portacaval shunts were not induced by subcutaneous splenic transposition (36), but constructed by the button technique (34) and bile duct ligations were performed in the same surgical session. This enabled the creation of a model of chronic liver failure with a minimum amount of surgical or other interventions and a relatively standardized and predictable portasystemic shunt. Rats were studied at 1 and 2 weeks after this procedure, since we previously showed that button shunted rats start to recover from several of the metabolic disturbances of portacaval shunting after 3-4 weeks (49), probably as a consequence of neovascularization and hepatopetal shunts $(38,49)$. Portacaval shunted rats without bile duct ligation and rats surgically treated as portacaval shunted rats, but in whom no shunt was constructed (sham operation), were studied in parallel. These latter two groups were pair fed to the portacaval 
shunted/biliary obstructed rats to rule out the effects of food intake as much as possible. The experimental work performed in this model will be presented in chapters $8,9,10 \& 11$.

\section{METHOD OF FLOW AND FLUX MEASUIREMENT}

Animals All experimental animal studies in this thesis were performed in male specified pathogen free Wistar rats (250-350 g, random bred Wistar/CPB:WU/Bor, Winkelmann, Borchen, Germany). They were housed under standard conditions (12 h light-dark cycle (light 7:30 a.m. - 7:30 p.m.)) and were not reallocated for at least one week prior to entering the experiments. Animals were fed standard lab chow and water ad libitum unless stated otherwise. Drinking water was acidified to $\mathrm{pH}=3$ to prevent bacterial or algae growth (routine procedure in our institute).

Materials Flow measurements were carried out under ether anesthesia (Diethylether, Chempure, Chempropack BV, Dordrecht, The Netherlands), except some

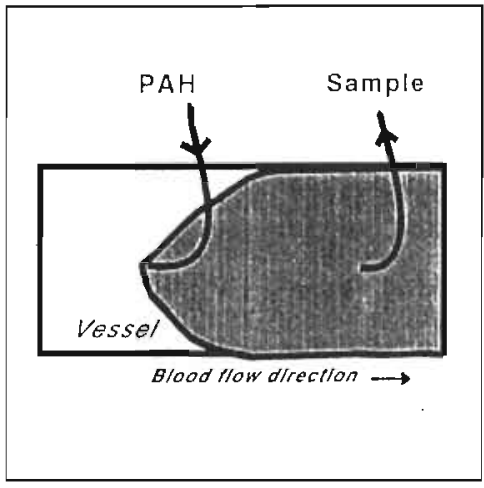

Figure 1: Flow measurement by the indicator dilution method. of the experiments reported under 'Cerebral cortex blood flow measurement' (chapter 6A), which were performed under ketamine (Ketamine $\mathrm{HCl}$, Nimatek ${ }^{\mathrm{R}}$, AUV Cuijck, The Netherlands) anesthesia. Flow measurements were carried out at constant core temperatures, monitored by an electronic rectal probe $\left(34 \pm 1^{\circ} \mathrm{C}\right.$ for acute liver ischemia rats, $37 \pm 1^{\circ} \mathrm{C}$ for all other rats). As outlined above, rats with acute liver ischemia develop hypothermia with core temperature dropping spontaneously to $31-34^{\circ} \mathrm{C}$.

For flow determinations, para-aminohippuric acid (PAH) solutions were used (Sigma A 1422 , St. Louis, MI, U.S.A.). These solutions were all iso-osmotic, and adjusted to $\mathrm{pH}=7.40$ and $\mathrm{T}=37^{\circ} \mathrm{C}$ before use. Catheters were always flushed with heparinized normal saline (50 IU $\mathrm{mL}^{-1}$, Heparine, Leo Pharmaceutische Produkten B.V., Emmen, The Netherlands).

PAH solutions were infused with the aid of infusion pumps (Minipuls 2 or Minipuls 3 pump, Gilson France SA, Villiers le Bel, France) at variable rates (1.0 to $6.5 \mathrm{~mL} \mathrm{~h}^{-1}$ ), depending on the organ under study.

Two types of silastic (Silastic Medical Grade Tubing, Dow Corning Corporation, Medical Products, Midland, MI, U.S.A.) needle bearing catheters were prepared. For blood sampling purposes, 25 Gauge needles were fitted to silastic tubing $(0.051 \mathrm{~cm}$ I.D., $0.094 \mathrm{~cm}$ O.D.), for PAH infusions $27 \mathrm{G}$ needles were fitted to silastic tubing $(0.031 \mathrm{~cm} \mathrm{I.D.,0.064 \textrm {cm }}$ O.D.). PE-50 (Intramedic ${ }^{\circledR}$ Polyethylene Tubing PE-50, $0.058 \mathrm{~cm} \mathrm{ID,} 0.096 \mathrm{~cm}$ OD, Clay Adams, Parsippany, NJ, U.S.A.) and PE-10 (0.030 cm I.D., $0.058 \mathrm{~cm}$ O.D.) tubing was used for infusions or sampling in several experiments.

Plasma flow measurements In all experiments reported in this thesis, flow measurements were performed using the indicator dilution method (57). This method is based on the indicator dilution principle (Figure 1): during infusion of an indicator into a vessel, at steady state the amount of indicator entering the body equals the amount excreted. In that situation, one can calculate the flow in the vessel if the infusion rate and the indicator concentration 
upstream and downstream of the infusion site are known. Otherwise stated: when e.g. black ink is thrown in a water stream, the intensity of the color downstream the place were the ink enters the stream depends on the rate at which the ink enters the stream as well as the flow in the stream. In a formula (58):

$$
F_{\text {argan }}=\frac{1}{[P A H]_{\text {post }}-[P A H]_{\text {pro }}}
$$

where $F_{\text {organ }}$ is the plasma flow $\left(\mathrm{mL} \mathrm{min}^{-1}\right)$ across the organ studied, I is the infused PAH $\left(\mu \mathrm{M} \mathrm{min}^{-1}\right)$, and $[\mathrm{PAH}]_{\text {post }}$ and $[\mathrm{PAH}]_{\text {pre }}$ are the concentrations of PAH $(\mu \mathrm{M})$ upstream and downstream of the infusion site. For all organs, except cerebral cortex, plasma flow is expressed per $100 \mathrm{~g}$ body weight (bw), since the weight of these organs can be expected to be intimately related to body weight.

As already stated, the calculation of plasma flows by this formula is only valid at steady state PAH concentrations. Steady state PAH concentrations were found to occur after 20 min of PAH infusion (Figure 2) in our model.

This method of flow measurement has extensively been used by others to study plasma flow across the portal drained viscera ( $\approx$ intestine) (59-63), the hindquarter $(\approx$ muscle) $(63-67)$, liver $(60,61,63)$ and kidney $(59,60,64,68)$. We have adapted and modified this method and developed a new method for measuring cerebral cortex plasma flow in the rat (chapter 6A).

Calculation of plasma flow Plasma fluxes were calculated as plasma flow times venous-arterial concentration differences, and expressed as nmol-100 g $\mathrm{bw}^{-1} \cdot \mathrm{min}^{-1}$ for all organs, except cerebral cortex (nmol $\mathrm{min}^{-1}$ ). A positive figure means net efflux, negative values reflect net uptake. The calculation of fluxes gives an estimate of net uptake by or release from an organ (Figure 3).
PAH equilibration studies
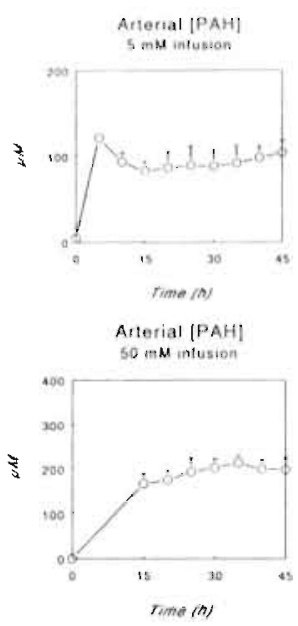

Figure 2: Steady state arterial PAH concentrations are obtained after a 20 min infusion period with either a $5 \mathrm{mM}$ (upper panel) or a $50 \mathrm{mM}$ solution (lower panel).

Principle of flux measurement

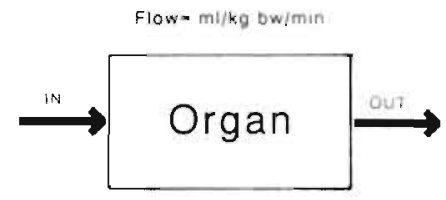

Flux $=$ flow * (OUT - IN)

Figure 3: Flux measurement gives information concerning net uptake or release, but not about unidirectional uptake or release.

\section{REFERENCES}

1. Sherlock S. Diseases of the liver and biliary system. Blackwell Sci Publ Oxford, 8th Edition, 1989.

2. de Knegt RJ, Schalm SW (1991). Fulminant hepatic failure: to transplant or not to transplant. Neth J Med 1991;38:131-141.

3. Hagenfeldt L, Eriksson LS, Wahren J. Amino acids in liver disease. Proc Nutr Soc 1983;42:497-506.

4. Zieve L. Pathogenesis of hepatic encephalopathy. Metab Brain Dis 1987;2:147-165. 
5. Drapanas T, McMenamy R.H, Adler WJ, Vang JO. Intermediary metabolism following hepatectomy in dogs. Ann Surg 1965:162:621-633.

6. Schalm SW, varı der Mey T. Hyperammonemic coma after hepatectomy in germ-free rats. Gastroenterology 1979;77:231-234.

7. James JH, Herlin PM, Edwards L, Nachbauer CA, Fisher JE. Effect of infusing the branched chain amino acids on concentrations of amino acids in plasma and on brain catecholamines after total hepatectomy in the rat. Life Sciences 1982;30:1361-1368.

8. Bosman DK, de Haan JG, Smit J, Jörning GGA, Maas MAW, Chamuleau RAFM. Metabolic activity of microcarrier attached liver cells after intraperitoneal transplantation during severe liver insufficiency in the rat. J Hepatol 1989:9:49-58.

9. Watanabe A, Shiota T, Takei N, Fujiwara M, Nagashima H. Ammonia detoxification by accelerated oxidation of branched chain amino acids in brains of acute hepatic failure rats. Biochem Med Metab Biol 1986;35:367-375.

10. Hanisch E, Holtmann G, Marhofer M, Berner V, Schweigkofler U, Paolucci V. Die portacavale Anastomosis im Lebercirrhosemodell bei der Ratte - Bedeutung einer speziellen preoperativen Vorbereitung zur Senkung der Operationsletalität. Langenbecks Arch Chir 1989;374:92-94.

11. Bassett ML, Mullen KD, Scholz B, Fenstermacher JD, Jones EA. Increased brain uptake of gammaaminobutyric acid in a rabbit model of hepatic encephalopathy. Gastroenterol 1990;98:747-757.

12. Albrecht J, Hilgier W. Arginine in thioacetamide-induced hepatogenic encephalopathy in rats: activation of enzymes of arginine metabolism to glutamate. Acta Neurol Scand 1986;73:498-501.

13. Rosado A, Flores G, Mora J, Soberon G. Distribution of an ammonia load in the normal rat. Am J Physiol 1962;203:37-42.

14. Hawkins RA, Miller AL, Nielsen RC, Veech RL. The acute action of ammonia on rat brain metabolism in vivo. Biochem J 1973;134:1001-1008.

15. Lin S, Raabe W. Ammonia intoxication: effects on cerebral cortex and spinal cord. J Neurochem 1985:44:1252-1258.

16. Hindfelt B, Plum F, Duffy TE. Effect of acute ammonia intoxication on cerebral metabolism in rats with portacaval shunts. J Clin Invest 1977;59:386-396.

17. Butterworth RF, Girard G, Giguère JF. Regional differences in the capacity for ammonia removal by brain following portocaval anastomosis. J Neurochem 1988;51:486-490.

18. Gjedde A, Lockwood AH, Duffy TE, Plum F. Cerebral blood flow and metabolism in chronically hyperammonemic rats: Effect of an acute ammonia challenge. Ann Neurol 1978;3:325-330.

19. Bosman DK, Deutz NEP, de Graaf AA, van der Hulst RWN, van Eijk HMH, Bovée WMMJ, Maas MAW, Jörning GGA, Chamuleau RAFM. Changes in brain metabolism during hyperammonemia and acute liver failure: Results of a comparative 'H-NMR spectroscopy and biochemical investigation. Hepatology 1990; 12:281-290.

20. Gibson GE, Zimber A, Krook L, Visek WJ. Nucleic acids and brain and intestinal lesions in ammonia intoxicated mice. Fed Proc 1971;30:578A (abstract).

21. Zimber A, Visek WJ. Effect of urease injections on DNA synthesis in mice. Am J Physiol 1972;223:1004-1008.

22. Cooper AJL, Mora SN, Cruz NF, Gelbard AS. Cerebral ammonia metabolism in hyperammonemic rats. J Neurochem 1985;44:1716-1723.

23. Deutz NEP. Chamuleau RAFM, de Graaf AA. Bovée WMMJ, de Beer R. In vivo ${ }^{31}$ P NMR spectroscopy of the rat cerebral cortex during acute hepatic encephalopathy. NMR Biomed 1988;1:101-106.

24. Mans AM, Saunders SJ, Kirsch RE, Biebuick JF. Correlation of plasma and brain amino acid and putative neurotransmitter alterations during acute hepatic coma in the rat. $J$ Neurochem 1979:32:285-292.

25. Butterworth RF, Swain M, Bergeron M, Blei AT. Cerebrospinal fluid amino acid changes in relation to neurological status in acute liver failure: studies in freely moving rats with in-dwelling cisterna magna catheters. Hepatology 1990; 12:857 (abstract).

26. Swain M, Butterworth RF, Blei AT. Brain amino acids and brain edema in acute liver failure. Hepatology 1990;12:858 (abstract).

27. Ganz R, Swain M, Traber P, DalCanto M, Butterworth RF, Blei AT. Ammonia induced swelling of rat cerebral cortex slices: implications for the pathogenesis of brain edema in acute hepatic failure. Metab Brain Dis 1989;4:213-223.

28. Bosman DK, van den Buijs ACG, de Haan JG, Maas MAW, Chamuleau RAFM. The effects of benzodiazepine-receptor antagonists and partial inverse agonists on acute hepatic encephalopathy in the rat. Gastroenterology 1991; 101:772-781. 
29. Chamuleau RAFM, Deutz NEP, de Haan JG, van Gool J. Correlation between electroencephalographic and biochemical indices in acute hepatic encephalopathy in rats. J Hepatol 1987;4:299-306.

30. Bosman DK, Deutz NEP, Maas MAW, van Eijk HMH, Smit JJH, de Haan JG, Chamuleau RAFM. Amino acid release from cerebral cortex in experimental acute liver failure, studied by in vivo cerebral cortex micro dialysis. J Neurochem 1992; in press.

31. Basile AS, Jones EA, Skolnick P. The pathogenesis and treatment of hepatic encephalopathy: evidence for the involvement of benzodiazepine receptor ligands. Pharmacol Rev 1991:43:27-71.

32. Mullen KD, Schafer DF, Cuchi P, Rössle M, Maynard TF, Jones EA. Evaluation of the suitability of galactosamine-induced fulminant hepatic failure as amodel of hepatic encephalopathy in the rat and rabbit, in: Advances in ammonia metabolism and hepatic encephalopathy, (Soeters PB, Wilson JHP, Meijer AJ, Holm E, eds.) Excerpta Medica, Amsterdam, pp. 205-2 12, 1988.

33. Lee SH, Fisher B. Portacaval shunt in the rat. Surgery 1961;50:668-672.

34. Funovics JM, Cummings MG, Shuman L, James JH, Fischer JE. An improved nonsuture method for portacaval anastomosis in the rat. Surgery 1975;77:661-664.

35. Swain M, Butterworth RF, Blei AT. Ammonia and related amino acids in the pathogenesis of brain edema in acute ischemic liver failure in rats. Hepatology 1992;15:449-453.

36. Maddison JE, Dodd PR, Morrison M, Johnston GAR, Fartell GC. Plasma GABA, GABA-like activity and the brain GABA-benzodiazepine receptor complex in rats with chronic hepatic encephalopathy. Hepatology 1987;7:621-628.

37. Kountouras J, Billing BH, Scheuer PJ. Prolonged bile duct obstruction: a new experimental model for cirrhosis in the rat. Br J Exp Path 1984;65:305-311.

38. Kyu MH, Cavanagh JB. Some effects of portocaval anastomosis in the male rat. Br J Exp Pathol 1970;51:217-227.

39. Mullen KD, McCullough AJ. Problems with animal models of chronic liver disease: Suggestions for improvement in standardization. Hepatology 1989;9:500-505.

40. Meijer AJ, Lamers WH, Chamuleau RAFM. Nitrogen metabolism and omithine cycle function. Physiol Rev 1990;70:701-748.

41. Record CO. Neurochemistry of hepatic encephalopathy. Gut 1991;32:1261-1263.

42. Kissinger JT, Griffin HE, Campbell SA, Hughes HC, Landi MS. A new portacval shunt. J Pharm Meth 1988;20:329-333.

43. Maddison JE, Watson WEJ, Dodd PR, Johnston GAR. Alterations in cortical $\left[{ }^{3} \mathrm{H}\right]$-kainate and $\alpha-\left[{ }^{3} \mathrm{H}\right]$ amino-3-hydroxy-5-methyl-4-isoxazolepropionic acid binding in a spontaneous canine model of chronic hepatic encephalopathy. J Neurochem 1991;56:1881-1888.

44. Imler M, Schlienger JL. The effect of chronic uremia on portal and systemic ammonemia in normal and portal-strictured rats. J Lab Clin Med 1979;94:872-878.

45. Imler M, Schlienger J-L, Chabrier G, Comte F. Origine rénale de l'hyperammoniémie provoquée par un régime hyperprotidique chez le rat normal ou porteur d'une stricture portale. Gastroenterol Clin Biol 1983;7:740-745.

46. Steele RD. Hyperammonemia and orotic aciduria in porta caval shunted rats. I Nutr 1984;114:210-216.

47. Peterson C, Giguère JF, Cotman CW, Butterworth RF. Selective loss of N-Methyl-D-Asparate sensitive $\mathrm{L}-\left[{ }^{3} \mathrm{H}\right]$-glutamate binding sites in rat brain following portacaval anastomosis. J Neurochem 1990;55:386-390.

48. Ribeiro J, Nordlinger B, Ballet F, Cynober L, Coudray-Lucas C, Baudrimont M, Legendre C, Delelo $\mathrm{R}$, Panis $\mathrm{Y}$. Intrasplenic hepatocellular transplantation corrects hepatic encephalopathy in portacaval shunted rats. Hepatology 1992;15:12-18.

49. de Boer JEG, Oostenbroek RJ, van Dongen JJ, Janssen MA, Soeters PB. Sequential metabolic characteristics following portacaval shunts in rats. Eur Surg Res 1986;18:96-106.

50. Butterworth RF, Le O, Lavoie J, Szerb JC. Effect portocaval anastomosis on electrically stimulated release of glutamate from rat hippocampal slices. J Neurochem 1991;56:1481-1485.

51. O'Connor MJ. Mechanical biliary obstruction. Amer Surg 1985;51:245-251.

52. Dunn CW, Horton JW, Megison SM, Vuitch MF. Contribution of portal systemic shunt to Kuppfer cell dysfunction in obstructive jaundice. J Surg Res 1991;50:234-239.

53. Roughneen PT, Gouma DJ, Kulkami AD, Fanslow WF, Rowlands BJ. Impaired specific cellmediated immunity in experimental biliary obstruction and its reversibility by intemal biliary drainage. J Surg Res 1986;41:113-125.

54. Greve JWM. Complications in obstructive jaundice. An experimental study on etiology and prevention. Thesis, Maastricht, The Netherlands, 1990. 


\section{Chapter 2}

55. Greve JWM, Gouma DJ, Soeters PB, Buurman WA. Suppression of cellular immunity in obstructive jaundice is caused by endotoxins. A study in germ-free rats. Gastroenterology 1990;98:478-485.

56. Stames HF, Conti PS, Warren RS, Jeevanandam M, Brennan MF. Altered peripheral amino acid uptake in obstructive jaundice. J Surg Res 1987;42:383-393.

57. Granger DN, Bulkley GB. Measurement of blood flow. Applications to the splanchnic circulation. Williams and Wilkins, London, $198 \mathrm{I}$.

58. Katz ML, Bergman EN. Simultaneous measurements of hepatic and portal venous blood flow in the sheep and dog. Am J Physiol 1969;216:946-952.

59. Welbourne TC, Phromphetcharat V, Givens G, Joshi S. Regulation of interorgan glutamine flow in metabolic acidosis. Am J Physiol 1986;250:E457-E463.

60. Phromphetcharat V, Welbourne TC. Renal glutamine extraction and gut/liver interaction in glutamine homeostasis. Contrib Nephrol 1985:47:9-14.

61. Buttrose M, McKellar D, Welbourne TC. Gut-liver interaction in glutamine homeostasis: portal ammonia role in uptake and metabolism. Am J Phys 1987;252:E746-E750.

62. Souba WW, Herskowotz K, Klimberg VS, Salloum RM, Plumley DA, Flynn TC, Copeland EM (1990). The effects of sepsis and endotoxemia on gut glutamine metabolism. Ann Surg 21 1: 543-551.

63. Schröck H, Goldstein L. Interorgan relationships for glutamine metabolism in normal and acidotic rats. Am J Physiol 1981;240:E519-E525.

64. Welbourne TC. Effect of metabolic acidosis on hindquarter glutamine and alanine release. Metabolism 1986;35:614-618.

65. Ardawi MSM. Skeletal muscle glutamine production in thermally injured rats. Clin Sci 1988:74:165-172.

66. Ardawi MSM, Jamal YS. Glutamine metabolism in skeletal muscle of glucocorticoid-treated rats. Clin Sci 1990;79:139-147.

67. Ruderman NB, Houghton CRS, Hems R. Evaluation of the isolated perfused rat hindquarter for the study of muscle metabolism. Biochem J 1971; 124:639-651.

68. Welboume TC, Dass PD. Gamma glutamyltransferase contribution to renal ammoniagenesis in vivo. Pt7ügers Arch 1988;411:573-578. 


\title{
Effects of starvation on interorgan amino acid and ammonia exchange ${ }^{1}$
}

Published as: C.H.C. Dejong, N.E.P. Deutz, P.B. Soeters. Inter-organ nitrogen exchange during prolonged starvation in the rat. J Clin Nutr Gastroenterol 1991,6:176-183.

\begin{abstract}
During starvation, enhanced nitrogen loss occurs from various organs. Glutamine and alanine have been suggested to be the main nitrogen carriers from peripheral to splanchnic tissues. In the liver, these amino acids are precursors for urea synthesis and thus can provide substrates for gluconeogenesis. To study inter-organ nitrogen transport during prolonged starvation, glutamine, alanine, ammonia and urea exchange was measured across the hindquarter, the portal drained viscera and the liver in male Wistar rats in the fed state or after 1, 2, 3 or 4 days of fasting.

Arterial urea increased in time $(\mathrm{P}<0.05)$, but glutamine, alanine and ammonia did not change. Hindquarter glutamine and alanine release increased and reached a maximum at day 3 , after which it declined. Simultaneously, portal drained viscera glutamine uptake, as well as alanine and ammonia release decreased at day 3 of starvation, while liver glutamine release switched to uptake at day 2 of starvation, coinciding with maximal urea release. In the course of starvation, an increasing proportion of hepatic urea release could be accounted for by hepatic uptake of blood derived glutamine, alanine and ammonia.

These results demonstrate that during prolonged starvation in the rat important differences exist in the pattern of glutamine and alanine exchange across muscle, gut and liver. The different time course of hepatic urea release and muscle glutamine and alanine release suggests that, besides muscle, other organs also are important in total body nitrogen loss during prolonged starvation in the rat.
\end{abstract}

\footnotetext{
'This chapter is an exact copy of the original publication and consequently does not contain all data from the experiments. Additional data are available on request.
} 


\section{INTRODUCTION}

In the past decades, considerable attention has been paid to research concerning nitrogen metabolism in various protein catabolic disease states, e.g. cancer (1), sepsis (2), (surgical) trauma (3), endotoxemia (2), pulmonary emphysema (4) and chronic liver disease (5). Only recently, interest has grown in the effects of pure starvation (6-11), probably arising from the understanding that starvation accompanies various disease states, including the peri-operative period $(7,9)$. Moreover, it was demonstrated that the nutritional status of muscle is an important determinant of some aspects of muscle response to sepsis (7), underlining the need to distinguish between the general effects of pure starvation and those of the specific disease.

During starvation, enhanced net protein catabolism leads to amino acid release from various organs $(3,9,10)$, probably for gluconeogenesis and the synthesis of essential proteins in the liver (7) and immune system. It has been suggested that protein catabolism mainly involves skeletal muscle proteolysis and wasting (7) and adversely affects physical activity, including respiratory function (4). In this context it has been shown that the composition of the amino acids released from muscle due to proteolysis (11) is not representative of skeletal muscle protein composition (12). The amino acids glutamine and alanine have been shown to constitute more than $50 \%$ of the amino acids released, although they constitute only $13 \%$ of skeletal muscle protein (12). For this reason, glutamine and alanine have been proposed to serve as carriers of carbon and nitrogen from skeletal muscle to splanchnic organs (12). These amino acids are taken up by the liver, their carbon skeletons serving as substrate for gluconeogenesis, and their nitrogen being processed in hepatic urea synthesis $(6,12)$.

Glutamine also constitutes the preferred fuel for the small intestine (13-16) and is hypothesized to be essential in the maintenance of gut integrity and mucosal barrier function (2). The nitrogenous breakdown products of intestinal glutamine metabolism are released into the portal vein as alanine and ammonia $(6,13-16)$ and further processed in the liver. This metabolic pathway provides more than $30 \%$ of the nitrogen precursors for hepatic urea synthesis in overnight fasted rats (14).

The present experiments were designed to investigate inter-organ nitrogen transport during prolonged starvation in the rat. We were specifically interested whether the pattern of glutamine and alanine release from peripheral tissues (i.e. skeletal muscle) corresponds stoichiometrically with the consumption of these metabolites in the splanchnic bed (i.e. liver plus intestines) and to hepatic urea synthesis. Knowledge about these aspects of the pathophysiology of starvation could be useful in nutritional support in several disease states associated with starvation (17). We therefore studied amino acid, ammonia and urea exchange across the hindquarter, the portal drained viscera and the liver during prolonged starvation in the rat.

\section{MATERIALS AND METHODS}

Animals Male SPF Wistar rats ( $\pm 300 \mathrm{~g}$, Winkelmann, Borchen, F.R.G.), individually housed in metabolism cages (12 hour light cycle, standard lab chow and water ad libitum), were used throughout. Prior to entering the experiment at day 0 , rats were allowed a 4 days acclimatization period in the metabolism cages. Rats were maintained in accordance with 
the recommendations of the Guide for the Care and Use of Laboratory Animals, as applied in our institute.

Experimental groups Rats were randomly assigned to one of the following five groups: Fed controls or 1, 2, 3 or 4 days fasted.

Sampling procedures (18) All sampling procedures were carried out under ether anesthesia at constant core temperatures $\left(37 \pm 1^{\circ} \mathrm{C}\right)$. Blood sampling and flow measurements were performed using the indicator dilution principle as described by Welbourne (19-21). Rats were anesthetized $30 \mathrm{~min}$ before sampling. A tertiary branch of the mesenteric vein, the portal vein, the hepatic vein, the inferior caval vein and the abdominal aorta were cannulated (both just above the bifurcation) with a $25 \mathrm{G}$ needle fitted to silastic tubing (Silastic Medical Grade Tubing $0.051 \mathrm{~cm} \mathrm{ID,} 0.094 \mathrm{~cm}$ OD, Dow Corning Corporation, Medical Products, Midland, MO, U.S.A.) and cemented in place with cyanoacrylate adhesive. For flow determinations, a primed constant infusion of para-aminohippuric acid (PAH) (50 mM, isomolaric, pH 7.40; Sigma A 1422, St. Louis, MO, U.S.A.) was started into the mesenteric and aorta catheters ( $1.5 \mathrm{~mL} \cdot \mathrm{h}^{-1}$ on each line; Minipuls 2 pump, Gilson France SA, Villiers le Bel, France) including a $20 \mathrm{~min}$ equilibration period. At the end of the equilibration period the right common carotid artery was catheterized (Intramedic Polyethylene Tubing PE-50, $0.058 \mathrm{~cm}$ ID, $0.096 \mathrm{~cm}$ OD, Clay Adams, Parsippany, NJ, U.S.A.). Blood samples were simultaneously drawn at a rate of $\pm 250 \mu \mathrm{L} \mathrm{min}^{-1}$ in heparinized cups on ice (Microvette LH CB1000, Sarstedt, Nümbrecht, F.R.G.) from the hepatic, portal and inferior caval vein and the carotid artery ( $750 \mu \mathrm{L} /$ catheter). Blood samples were kept on ice during transport to the laboratory where further processing within 30 minutes before storage at $-70{ }^{\circ} \mathrm{C}$ was performed.

The above described catheter positions combined with the infusion of PAH permit simultaneous determination of blood flow and arteriovenous concentration difference (=flux) across the portal drained viscera $(2,19,20,22)$, the liver and the hindquarter $(21,23,24)$. This technique of metabolite exchange measurement across the portal drained viscera, the liver and the hindquarter has been extensively used to study intestinal $(1,2,19,20,22,25)$, hepatic $(20,22,25)$ and skeletal muscle metabolism in the rat $(21,23-26)$.

Biochemical analysis Centrifugation was performed at $4^{\circ} \mathrm{C}$ during 5 minutes at $8,800 \mathrm{~g}$. All samples were stored at $-70^{\circ} \mathrm{C}$. Hematocrit was obtained with a microfuge. Ammonia and urea were determined enzymatically in plasma as described previously $(18,27)$. Plasma glutamine and alanine were determined by HPLC technique (28). For PAH-determination in whole blood the method according to Brun was modified $(18,27,29)$. The coefficient of variance was $2 \%$ for glutamine, $3 \%$ for alanine, $4 \%$ for ammonia and $2 \%$ for urea $(18,28)$.

Calculations Plasma PAH concentration was calculated from whole blood PAH concentrations using the hematocrit. Plasma flow was calculated as follows $(18,30)$ :

$$
F_{L P O Y H O}=\frac{1}{[P A H]_{\text {IOY HY PV }}-[P A H]_{A}}
$$

In this formula, $\mathrm{F}_{\mathrm{L}, \mathrm{PDV} . \mathrm{HQ}}$ is the liver, portal drained viscera and hindquarter plasma flow respectively $\left(\mathrm{mL} \cdot \mathrm{min}^{-1}\right), \mathrm{I}$ is the infused PAH $\left(\mu \mathrm{mol} \mathrm{min}^{-1}\right)$, and $[\mathrm{PAH}]_{\mathrm{ICV}, H V . P V . A}$ is the plasma PAH concentration $(\mu \mathrm{M})$ in the inferior caval vein, the hepatic vein, the portal vein and the carotid artery, respectively. 
Table. Body weight, hindquarter, portal drained viscera and liver plasma flows and arterial metabolite concentrations during prolonged starvation in the rat.

\begin{tabular}{|c|c|c|c|c|c|c|c|c|c|c|c|}
\hline \multirow[b]{3}{*}{ Body weight } & \multirow{2}{*}{\multicolumn{2}{|c|}{$\begin{array}{l}\text { FED } \\
0 \text { day }(9)\end{array}$}} & \multicolumn{8}{|c|}{ FASTED } & \multirow{3}{*}{$\frac{\text { Sign. }}{P<0.0001}$} \\
\hline & & & \multicolumn{2}{|c|}{$1 \operatorname{day}(10)$} & \multicolumn{2}{|c|}{2 davs $(10)$} & \multicolumn{2}{|c|}{3 days $(10)$} & \multicolumn{2}{|c|}{4 days $(10)$} & \\
\hline & $331 \pm$ & 6 & $297 \pm$ & 3 & $285 \pm$ & 3 & $269 \pm$ & 4 & $265 \pm$ & 5 & \\
\hline HQ flow & $1.9 \pm$ & $\$ .1$ & $1.2 \pm$ & 0.5 & $1.7 \pm$ & 0.9 & $1.9 \pm$ & 0.9 & $0.6 \pm$ & 0.2 & NS \\
\hline PDV flow & $1.5 \pm$ & 0.8 & $1.4 \pm$ & 0.7 & $1.9 \pm$ & 0.7 & $0.9 \pm$ & 0.4 & $1.0 \pm$ & 0.3 & NS \\
\hline Liver flow & $1.9 \pm$ & 1.1 & $1.1 \pm$ & 0.5 & $3.9 \pm$ & 1.3 & $1.2 \pm$ & 0.5 & $1.7 \pm$ & 0.4 & NS \\
\hline Glutamine & $599 \pm$ & 25 & $560 \pm$ & 29 & $548 \pm$ & 24 & $530 \pm$ & 32 & $590 \pm$ & 24 & NS \\
\hline Alanine & $282 \pm$ & 25 & $353 \pm$ & 32 & $291 \pm$ & 25 & $329 \pm$ & 45 & $342 \pm$ & 36 & NS \\
\hline Ammonia & $91 \pm$ & 15 & $88 \pm$ & 17 & $74 \pm$ & 9 & $69 \pm$ & 14 & $83 \pm$ & 12 & NS \\
\hline Urea & $7.4 \pm$ & 0.5 & $6.9 \pm$ & 0.4 & $7.0 \pm$ & 0.2 & $8.3 \pm$ & 0.4 & $8.8 \pm$ & 0.9 & $P=0.05$ \\
\hline Melab \% urea & $22 \pm$ & 8 & $16 \pm$ & 4 & $33 \pm$ & 14 & $47 \pm$ & 20 & $91 \pm$ & 14 & $P<0.001$ \\
\hline
\end{tabular}

Data are means $\pm S E M$. Statistics: analysis of variance (ONEWAY procedure). Number of rats in parentheses. Body weight in grams, plasma flows in $\mathrm{mL} \cdot 100 \mathrm{~g}$ body weight $\mathrm{t}^{-1} \cdot \mathrm{min}^{-1}$, ammonia, glutamine and alanine in $\mu \mathrm{mol} \mathrm{L}^{-1}$, urea in mmol $\mathrm{L}^{-1}$. Metab $\%$ urea: the percentage of hepatic urea release that can be accounted for by the sum of liver nitrogen fluxes of glutamine, alanine and ammonia.

Hindquarter and portal drained viscera fluxes ( $\mathrm{nmol} \cdot 100 \mathrm{~g}$ body weight ${ }^{-1} \mathrm{~min}^{-1}$ ) were calculated as mean plasma flow per group times individual inferior caval venous-arterial and portal vein-arterial concentration difference, respectively. Mean flows were used instead of individual flows, since not for all animals flows were available. Total splanchnic flux was calculated as mean group hepatic plasma flow times individual hepatic-arterial concentration difference, liver flux was calculated by subtracting portal drained viscera flux from total splanchnic flux, as described previously (27). A positive figure means net efflux, negative values reflect net uptake.

Statistics Data presented are means $\pm \mathrm{SEM}, \mathrm{P}<0.05$ was considered significant. For statistical analysis (31), analysis of variance (ONEWAY procedure) and Mann-Whitney $U$ non-parametric test were used where appropriate.

\section{RESULTS}

General (Table) During the period of adaptation to individual housing in the metabolic cages, all rats gained weight (data not shown). During starvation weight loss finally amounted to $20 \%$ of body weight, in agreement with literature data (11). Plasma flows across the various organs did not change significantly in time.

Arterial values (Table) Arterial glutamine, alanine and ammonia did not change, but arterial urea increased during starvation. Thus, the plasma glutamine pool is maintained within narrow limits in our experiments.

Glutamine and alanine fluxes (Figure I) Hindquarter glutamine and alanine release (Figure 1, upper panel) in $24 \mathrm{~h}$ fasted rats were in the same order as $(21,25)$ or considerably lower than $(23,24)$ data reported in literature. The discrepancies are due mainly to higher 
hindquarter plasma flow in these studies $(23,24)$. Glutamine and alanine release reached a maximum after 3 days starvation, after which it declined $(\mathrm{P}<0.01)$ in relation with a tendency to diminished-hindquarter plasma flow. At any time point, glutamine and alanine together accounted for at least $50 \%$ of all amino acids released from skeletal muscle (data not shown).

In all groups, portal drained viscera ( $\approx$ intestine) glutamine uptake and alanine release (Figure 1, middle panel) was observed, in amounts approximately comparable to previous results obtained by others $(2,19,20$, $22,25)$ as well as in our lab in overnight fasted rats (18). Glutamine uptake remained unchanged during the first two days of starvation, but decreased at day 3 and 4 of starvation $(\mathrm{P}<0.001)$. This was reflected in a decrease in alanine release after day 2 of starvation $(\mathrm{P}<0.05)$.

Hepatic fluxes of all metabolites were less than reported in the literature $(19,20,25)$. This can be largely explained by a lower hepatic plasma flow in our studies, which we cannot explain. At the second day of starvation, liver exchange switched from glutamine release to uptake $(\mathrm{P}<0.0001)$, alanine was taken up continuously (Figure 1, lower panel).

Ammonia and urea fluxes (Figure 2) Hindquarter ammonia flux (Figure 2, upper panel) was not significantly different from zero, except in the 3 days starved group $(p=0.035)$, coinciding with the above described maximal glutamine and alanine release. Portal drained viscera ammonia release (Figure 2, middle panel) followed a course opposite to glutamine uptake, as could be expected; thus, ammonia release by the portal drained viscera decreased after day 2 of starvation $(\mathrm{P}<0.0001)$. Hepatic ammonia uptake (Figure 2, lower panel) did not change in time, but urea release increased to a maximum at day 2 of the starvation period $(\mathrm{P}<0.05)$ (Figure 2, lower panel). This maximal urea release coincided with the switch in liver glutamine handling from release to uptake.

For a correct interpretation of these data, a calculation of 'nitrogen balance' across the liver seems valuable. Calculation of the total nitrogen content of hepatic ammonia, glutamine and alanine flux as a percentage of hepatic urea nitrogen release, gives an indication of the amount of glutamine, alanine and ammonia potentially utilized in urea synthesis. Such a calculation shows that the potential contribution of these metabolites to urea formation increases progressively in the course of starvation (from $22 \%$ in fed rats to $91 \%$ in 4 days starved rats; $\mathrm{P}<0.001$ ) (Table).

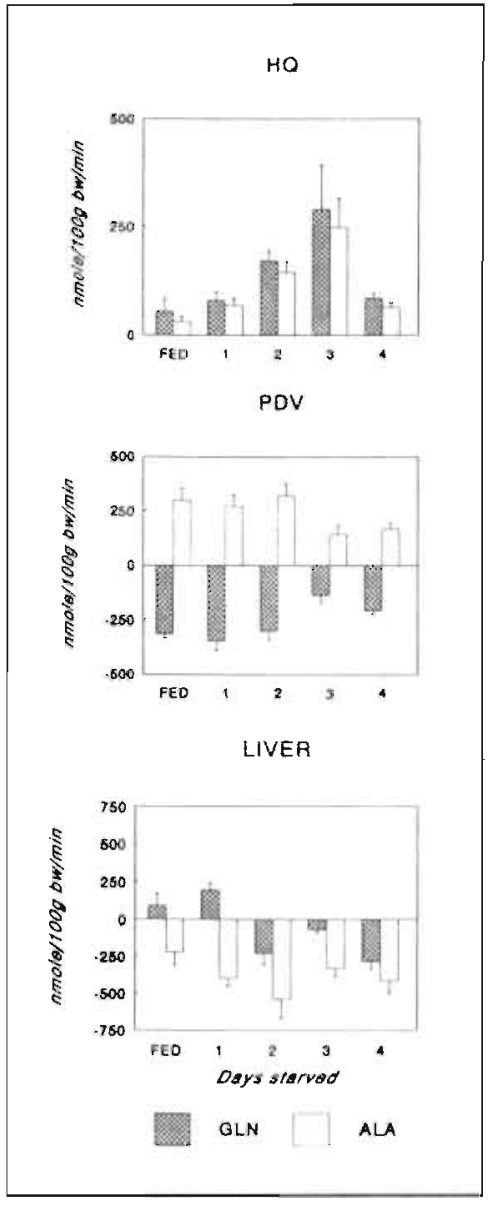

Figure 1. Glutamine (shaded bars) and alanine (open bars) plasma fluxes across the hind quarter, mainly representative of skeletal muscle metabolism (upper panel), the portal drained viscera, mainly representing intestinal metabolism (middle panel) and liver (lower panel) during prolonged starvation in the rat. Data are means $\pm S E M$ in $n \mathrm{~mol} \cdot 100 \mathrm{~g}$ bw $w^{-1} \min ^{-1}$. 
With respect to the splanchnic bed as a whole, net glutamine, alanine and ammonia uptake and urea release occurred in this region in virtually all groups during prolonged starvation (not shown). Exceptions were alanine flux in fed rats and ammonia fluxes in fed and 2 days starved rats, which were not significantly different from zero.

\section{DISCUSSION}

In the present experiments, we wanted to investigate the effects of prolonged starvation on inter-organ glutamine and alanine coupled nitrogen exchange. For this purpose we measured hindquarter, portal drained viscera and hepatic fluxes of glutamine, alanine, ammonia and urea in fed rats and rats fasted for varying periods of time. We were especially interested in the pattern of metabolite release or consumption by the above described organs.

During starvation, apart from effects on fat and glycogen stores, three phases of nitrogen loss in the rat can be distinguished (11). Phase 1, early starvation (24$48 \mathrm{~h}$ ), is characterized by accelerated proteolysis and nitrogen loss. In phase $2(48-120 \mathrm{~h})$, prolonged starvation, the animal conserves protein and finally, in phase 3 , terminal starvation, protein catabolism is again enhanced. Depending on their protein turnover rate, the relative contribution of various organs to total body protein catabolism and inter-organ nitrogen fluxes has been suggested to vary at different time points in the course of prolonged starvation $(3,10,32)$. Similarly, different types of proteins might contribute to total body catabolism at different time points depending on their turnover rates (11).

The present experiments show that after 2 days of starvation glutamine consumption by the portal drained viscera, mainly representing intestinal metabolism, decreases by $40-50 \%$. This diminished gut glutamine consumption is expectedly paralleled by a decreased intestinal release of ammonia and alanine, two principal precursors of hepatic urea synthesis and intimately linked to gluconeogenesis.

Decreased intestinal alanine release, as assessed by $\mathrm{P}-\mathrm{A}$ concentration difference measurement, has been described previously after $48 \mathrm{~h}$ starvation in the rat (6). However, in that study ( 6 ) glutamine P-A difference remained unchanged, and flow was not measured. In this context it must be taken into account that glutamine is not only extracted from blood, but also from the gut lumen (15). Thus, even if the glutamine flux remains unchanged during 
early starvation, this does not take into account that fed rats probably metabolize additional glutamine taken up from the lumen. The unchanged glutamine extraction by the gut during early starvation could therefore indicate an already decreased total intestinal glutamine consumption.

Glutamine and alanine release by the hindquarter increased progressively during starvation, in agreement with the hypothesis that organs with a low protein tumover rate increasingly contribute to total body proteolysis during prolonged starvation $(3,10,32)$. The sudden decrease at day 4 of starvation may constitute an attempt to conserve protein (17). Alternatively, muscle mass and muscle glutamine or available substrates might have become depleted, muscle glutamine release may have become inhibited or the activity of glutamine synthetase may have decreased (4), e.g. through decreased de novo synthesis of the enzyme. Although we think muscle glutamine depletion is the most likely explanation, a clear-cut conclusion cannot be drawn from our experiments.

At day 2 of starvation, hepatic urea release increased to a maximum after an initial decrease at day 1 . This seems to be in agreement with starvation-induced increased urea cycle enzyme activity and increased urinary urea excretion after 3 days starvation $(17,33)$. The maximal urea release coincided with the reversal of hepatic glutamine release to uptake and with a maximal alanine uptake at day 2 . The switch to hepatic glutamine uptake could indicate that other energy substrates, derived from glycogen and/or fat breakdown, gradually have become depleted. This seems to be supported by the observation that an increasing proportion of hepatic urea release could be accounted for by blood-derived liver glutamine, alanine and ammonia uptake, suggesting that these metabolites increasingly contribute to hepatic urea synthesis, intimately linked to gluconeogenesis. This could mean that either nitrogen precursors from intra-cellular hepatic stores are not available for urea synthesis or alternatively that blood derived precursors are preferentially used for urea synthesis in the course of starvation.

Our experiments show that in the fed state as well as during starvation muscle proteolysis produces glutamine as well as alanine, and the splanchnic area consumes these metabolites. This ultimately results in hepatic urea release, probably serving gluconeogenesis. This suggests that muscle proteolysis and subsequent nitrogen loss contributes to hepatic urea synthesis. However, the time course of hepatic urea release differs from muscle alanine and glutamine release, maximal glutamine and alanine release being $24 \mathrm{~h}$ later than maximal hepatic urea synthesis. This suggests an other organ consuming glutamine and alanine, since arterial glutamine and alanine concentrations did not increase. The kidney seems a good candidate for this role, since it can contribute to gluconeogenesis and is able to excrete ammonia.

The present experiments demonstrate that during prolonged starvation in the rat important differences exist in the pattern of glutamine and alanine exchange across muscle, gut and liver. The different time course of hepatic urea release and muscle glutamine and alanine release suggest that, besides muscle, other organs also are important in total body nitrogen loss during prolonged starvation in the rat. Future studies in our lab are directed towards this and related problems. 


\section{REFERENCES}

1. Souba WW, Strebel FR, Bull JM, Copeland EM, Teagtmeyer H, Cleary K. Interorgan glutamine metabolism in the tumor-bearing rat. J Surg Res 1988:44:720-726.

2. Souba WW, Herskowotz K, Klimberg VS, Salloum RM, Plumley DA, Flynn TC, Copeland EM. The effects of sepsis and endotoxemia on gut glutamine metabolism. Ann Surg 1990;211:543-551.

3. Rennie MJ, Bennegard K, Eden E, Emery PW, Lundholm K. Urinary excretion and efflux from the leg of 3-methylhistidine before and after major surgical operation. Metabolism 1984;33:250-256.

4. Morrison WL, Gibson JNA, Scrimgeour C, Rennie MJ. Muscle wasting in emphysema. Clin Sci 1988;75:415-420.

5. Rudman D, DiFulco TJ, Galambos JT, Smith RB, Salam AA, Warren WD. Maximal rates of excretion and synthesis of urea in normal and cirrhotic subjects. J Clin Invest 1973;52:2241-2249.

6. Kimura RE, Lapine TR, Johnston J, Ilich JZ. The effect of fasting on rat portal venous and aortic blood glucose, lactate, alanine and glutamine. Ped Res 1988;23:24 I-244.

7. Warner BW, James JH, Hasselgren PO, Hummel RP, Fischer JE. Effect of sepsis and starvation on amino acid uptake in skeletal muscle. J Surg Res 1987;42:377-382.

8. Pardridge WM. Unidirectional influx of glutamine and other neutral amino acids into liver of fed and fasted rats in vivo. Am J Physiol 1977;232:E492-E496.

9. Wamer BW, Hummel RP, Hasselgren PO, James JH, Fischer JE. Inhibited amino acid uptake in skeletal muscle during starvation. JPEN 1989;13:344-348.

10. Goodman MN, Larsen PR, Kaplan MM, Aoki TT, Young VR, Ruderman NB. Starvation in the rat. II. Effect of age and obesity on protein sparing and fuel metabolism. Am J Physiol 1980;239:E277-E286.

11. Lowell BB, Ruderman NB, Goodman MN. Regulation of myofibrillar protein degradation in rat skeletal muscle during brief and prolonged starvation. Metabolism 1986;35:1121-1127.

12. Ruderman NB, Berger M. The formation of glutamine and alanine in skeletal muscle. J Biol Chem $1974 ; 249: 5500-5506$.

13. Windmueller HG, Spaeth AE. Identification of ketone bodies and glutamine as the major respiratory fuels in vivo for postabsorptive rat small intestine. J Biol Chem 1978:253:69-76.

14. Windmueller HG. Glutamine utilization by the small intestine. Adv Enzyme 1982;53:210-237.

15. Windmueller $\mathrm{HG}$, Spaeth AE. Intestinal metabolism of glutamine and glutamate from the lumen as compared to glutamine from blood. Arch Bioch Bioph 1975;171:662-672.

16. Windmueller HG, Spaeth AE. Uptake and metabolism of plasma glutamine by the small intestine. J Biol Chem 1974;249:5070-5079.

17. Hatchwell LC, Milner JA. Factors affecting amino acid induced orotic aciduria in rats. J Nutr 1978; 108:1976-1981.

18. Dejong CHC, Kampman MT, Deutz NEP, Soeters PB. Altered glutamine metabolism in rat portal drained viscera and hindquarter during hyperammonemia. Gastroenterology 1992;102:936-948.

19. Welbourne TC, Phromphetcharat V, Givens G, Joshi S. Regulation of interorgan glutamine flow in metabolic acidosis. Am J Physiol 1986;250:E457-E463.

20. Phromphetcharat V, Welboume TC. Renal glutamine extraction and gut/iver interaction in glutamine homeostasis. Contr Nephrol 1985;47:9-14.

21. Welboume TC. Effect of metabolic acidosis on hindquarter glutamine and alanine release. Metabolism 1986:35:614-618.

22. Buttrose M, McKellar D, Welboume TC. Gut-liver interaction in glutamine homeostasis: portal ammonia role in uptake and metabolism. Am J Phys 1987;252:E746-E750.

23. Ardawi MSM. Skeletal muscle glutamine production in thermally injured rats. Clin Sci 1988;74:165-172.

24. Ardawi MSM, Janal YS. Glutamine metabolism in skeletal muscle of glucocorticoid-treated rats. Clin Sci 1990;79:139-147.

25. Schröck H. Goldstein L. Interorgan relationships for glutamine metabolism in normal and acidotic rats. Am J Physiol 1981;240:E519-E525.

26. Ruderman NB, Houghton CRS, Hems R. Evaluation of the isolated perfused rat hindquarter for the study of muscle metabolism. Biochem J 1971;124:639-651.

27. Deutz NEP, Reijuen PLM, Bost MCF, van Berlo CLH, Soeters PB. Modifications of the effects of blood in the gut by intravenous isoleucine. Gastroenterology 1991;101:1613-1620.

28. van Eijk HMH, van der Heijden MAH, van Berlo CLH, Soeters PB. Fully automated liquidchromatographic determination of amino acids. Clin Chem 1988;34:2510-2513. 
29. Brun C. A rapid method for the determination of para-aminohippuric acid in kidney function tests. J Lab Clin Med 1951;37:955-958.

30. Katz ML, Bergman EN. Simultaneous measurements of hepatic and portal venous blood flow in the sheep and dog. Am J Physiol 1969;216:946-952.

31. Norusis MJ ed. SPSS/PC+ V3.I BASE MANUAL for the IBM PC/XT/AT and PS/2. SPSS Inc, Chicago, USA, 1989.

32. Wassner SJ, Li JB. N-methylhistidine release: contributions of rat skeletal muscle, GI tract and skin. Am J Physiol 1982;243:E293-E297.

33. Schimke RT. Differential effects of fasting and protein free diets on levels of urea cycle enzymes in rat liver. J Biol Chem 1962;237:1921-1924. 



\title{
Effects of enterectomy on interorgan amino acid and ammonia exchange ${ }^{1}$
}

Published as: N.E.P. Deutz, C.H.C. Dejong, G. Athanasas, P.B. Soeters. Partial enterectomy in the rat does not diminish muscle glutamine production. Metabolism 1992;41:in press.

\begin{abstract}
The hypothesis was posed that consumption of the amino acid glutamine by the splanchnic tissues is an important regulating mechanism for its production in muscle. Therefore, glutamine consumption or production in portal-drained viscera, liver and hindquarter was measured by determining fluxes and intra-cellular concentrations after $80 \%$ enterectomy or SHAM operation in rats. Moreover, fluxes and intra-cellular concentrations of several other amino acids, ammonia and liver urea production were determined concomitantly.

After enterectomy, arterial glutamine concentration was increased, portal-drained viscera glutamine consumption was decreased by $80 \%$ and liver glutamine consumption was unchanged, compared to SHAM operated rats. Although hindquarter glutamine production remained unchanged after enterectomy, intra-cellular glutamate concentration (glutamine precursor) was lower, suggesting that enterectomy induces changes in muscle metabolism without changing the flux of glutamine. For the remaining gut, it was calculated that after enterectomy, glutamine consumption per gram remaining gut tissue increased. These results cast doubt on the hypothesis that diminished splanchnic glutamine uptake can reduce muscle glutamine production.
\end{abstract}

\section{INTRODUCTION}

In the transport of waste nitrogen from peripheral to splanchnic tissues, the amino acids glutamine and alanine are of crucial importance (1). In catabolic states, muscle glutamine

\footnotetext{
'This chapter is an exact copy of the original publication and consequently does not contain all data from the experiments. Additional data are available on request.
} 
concentration rapidly falls and glutamine release from muscle increases (2-8). In this situation, intestinal glutamine uptake (9-11) and hepatic glutamine and alanine uptake $(9,10)$ are also enhanced. Glutamine, produced in the peripheral tissues is thought to provide nitrogen and carbon skeletons, essential for proper functioning of gut and liver.

The gut primarily uses glutamine as an energy source $(1,12)$ and the nitrogenous products of this glutamine degradation provide the liver with substrates for urea synthesis $(10,12,13)$. Apart from the intestinal breakdown products of glutamine, the liver also takes up glutamine and alanine directly and utilizes them in urea synthesis and gluconeogenesis $(10,14)$.

Recently, the hypothesis was proposed that consumption of glutamine by the splanchnic tissues regulates its production in muscle (15). Human studies seemed to support this hypothesis because in two septic patients with a high leg muscle glutamine release, glutamine release was diminished after major enterectomy (8). These and other observations (16) suggest that removal of a glutamine consuming organ (e.g. small intestine) lowers the total body glutamine consumption and consequently reduces the need for peripheral glutamine production.

To study this hypothesis in rats, we measured amino acid, ammonia and urea consumption or production of gut, liver and muscle by determining fluxes and intra-cellular concentrations of these organs after $80 \%$ enterectomy or SHAM operation in rats. The results of our study do not support the hypothesis that diminished splanchnic uptake reduces muscle glutamine production. However, small bowel resection induced changes in liver and muscle intra-cellular amino acid concentrations.

\section{MATERIALS AND METHODS}

Animals Male SPF Wistar rats $(\mathrm{n}=42,250 \pm 25 \mathrm{~g}$, random bred Wistar/CPB:WU/Bor, Winkelmann, Borchen, F.R.G.) were used throughout. Rats were housed under standard conditions (not transferred for at least 1 week, 12 hour light cycle: 7.30 a.m - 7.30 p.m.) and fed standard lab chow until the evening before surgery and water ad libitum. Drinking water was acidified to $\mathrm{pH}=3$ to prevent bacterial and algae growth. Rats were maintained and humanely cared for in accordance with the recommendations of the Guide for the Care and Use of Laboratory Animals, as applied in our institute.

Experimental groups Rats were assigned randomly to one of four groups. The first group ( $\mathrm{CON} 1, \mathrm{n}=9$ ) consisted of normal control rats, in which flux measurement and tissue sampling after an overnight fast was performed. The second group (CON2, $n=11$ ) contained rats that were starved for $40 \mathrm{~h}$ (overnight fast and $24 \mathrm{~h}$ ), a period of starvation similar to that of the two remaining experimental groups (ENT and SHAM: see below). These two control groups (CON1 and CON2) were included to check for the effects of pure starvation. The third group (ENT, $n=11$ ) was composed of rats in which a $80 \%$ enterectomy was done after an overnight fast. These rats were allowed to recover from surgery and were then kept fasted until the next day, when flux measurements and tissue sampling was done (total duration of starvation: 40h). Finally, the fourth group (SHAM, $\mathrm{n}=11$ ) consisted of rats in which a SHAM enterectomy (see below) was done after an overnight fast. These rats also were allowed to recover and kept fasted until the next day (see above). Water was administered ad libitum throughout the experiments. 
Surgical procedures All operations and sampling procedures were performed at room temperature under ether anesthesia. Enterectomy was performed by removing $80 \%$ of the small intestine, leaving approximately $8 \mathrm{~cm}$ of the terminal ileum and $8 \mathrm{~cm}$ of the proximal jejunum. An anastomosis was made between jejunum and ileum with a single running silk 7 0 suture. SHAM operation was performed by cutting and re-anastomosing with a single running silk 7-0 suture randomly either at the ileum or at the jejunum. After this procedure, the animals were allowed to recover and transferred back to their cages.

Blood/ tissue sampling and flow measurements Rats were anesthetized $30 \mathrm{~min}$ before sampling. Rectal temperature was monitored using an electronic temperature probe and maintained at pre-anesthesia levels, using a heating lamp, heating pads, and moist saline gauzes. The portal vein, a small mesenteric vein, the hepatic vein, the inferior caval vein and the abdominal aorta were cannulated (both just above the bifurcation) with a $25 \mathrm{G}$ needle fitted to silastic tubing (Silastic Medical Grade Tubing $0.051 \mathrm{~cm} \mathrm{ID}, 0.094 \mathrm{~cm}$ OD, Dow Corning Corporation, Medical Products, Midland, MO, U.S.A) and cemented in place with cyanoacrylate adhesive.

For flow determinations, para-aminohippuric acid (PAH: $5 \mathrm{mM}$, iso-osmolaric, $\mathrm{pH}$ 7.40) (Sigma A 1422, St. Louis, MO, U.S.A.) was infused during a 20 min equilibration period at a rate of $1.5 \mathrm{~mL} \cdot \mathrm{h}^{-1}$ in the aorta and mesenteric vein (Minipuls 2 pump, Gilson France SA, Villiers le Bel, France) after a priming dose $\left(0.15 \mathrm{~mL} \cdot 100 \mathrm{gr} \mathrm{bw}^{-1}\right)$ of a $50 \mathrm{mM}$ $\mathrm{PAH}$ solution. At the end of the equilibration period the right common carotid artery was catheterized (Intramedic Polyethylene Tubing PE 50, $0.058 \mathrm{~cm} \mathrm{ID}, 0.096 \mathrm{~cm} \mathrm{OD,} \mathrm{Clay}$ Adams, Parsippany, NJ, U.S.A.).

Blood was slowly and simultaneously aspirated $(1.0 \mathrm{~mL}$ per vessel at a rate of 1.0 $\mathrm{mL} \min ^{-1}$ ) from the portal, hepatic and inferior caval vein and the common carotid artery. Immediately after sampling, the right gastrocnemius muscle, a piece of the left liver lobe and a piece of jejunum, just distal to the duodenojejunal ligament, were rapidly excised, freeze clamped with Wollenberg tongs (17) (total procedure in less than 30 seconds), put into liquid nitrogen, and stored at $-70^{\circ} \mathrm{C}$. At the end of the experiment rats were killed by an overdose of the anesthetic. The procedures of blood and tissue sampling were identical in all groups.

Biochemical analysis During all procedures, accomplished promptly after collection of blood, the samples were kept on ice. Within 10 min centrifugation was performed at $4^{\circ} \mathrm{C}$ at $8,500 \mathrm{~g}$ during $5 \mathrm{~min}$. Hematocrit was obtained with a microfuge. Plasma was obtained by whole blood centrifugation in heparinized tubes (Microvette LH CB1000, Sarstedt, Nümbrecht, F.R.G.) and frozen in liquid nitrogen immediately (ammonia) or after deproteinization (for amino acids: $100 \mu \mathrm{L}$ of plasma with $4 \mathrm{mg}$ sulfosalicylic acid (SSA)), after which the samples were stored at $-70^{\circ} \mathrm{C}$. For PAH determinations $100 \mu \mathrm{L}$ of whole blood was deproteinized with $400 \mu \mathrm{L}$ of $10 \%$ trichloracetic acid, thoroughly mixed, allowed to stand for deproteinization $\left(4^{\circ} \mathrm{C}, 30 \mathrm{~min}\right)$ and centrifuged after which the supernatant was collected and stored at $-70^{\circ} \mathrm{C}$ until further analysis. Spectrophotometric determinations (ammonia, urea, PAH) were done on a centrifugal analyzer system (Cobas Bio, Roche, Basle, Switzerland). Ammonia (18) and urea were determined by standard enzymatic methods using commercial kits. Urea values were corrected for ammonia. For PAH determination, the supernatant obtained as described above, was deacetylated by heating for $45 \mathrm{~min}$ at $100^{\circ} \mathrm{C}$ and the method according to Brun (19) was modified for small volumes. Amino acids were determined by HPLC technique as described previously (20). The coefficient of 
Table 1. Arterial concentrations.

\begin{tabular}{|c|c|c|c|c|c|c|c|c|c|c|c|c|c|}
\hline & & CON1 & $p$ & & $\mathrm{CON} 2$ & $P$ & & HAM & $P$ & & ENT & & $P$ \\
\hline$\overline{A m m}$ & 86 & \pm 15 & & 58 & \pm & 7 & 48 & \pm & $2^{2}$ & 88 & \pm & 14 & $y$ \\
\hline Urea & 7.1 & \pm 0.6 & & 7.1 & \pm & 0.5 & 8.0 & \pm & 0.5 & 9.5 & \pm & 0.5 & $m$ \\
\hline GLU & 78 & \pm 7 & 7 & 59 & \pm & $6^{1}$ & 59 & \pm & $4^{1}$ & 59 & \pm & 7 & \\
\hline ASN & 48 & \pm 4 & 4 & 41 & \pm & 2 & 40 & \pm & 3 & 51 & \pm & 8 & \\
\hline SER & 209 & $\pm \quad 17$ & & 165 & \pm & $6^{1}$ & 156 & \pm & $6^{2}$ & 175 & \pm & 17 & 1 \\
\hline GLN & 572 & $\pm \quad 47$ & & 556 & \pm & 23 & 581 & \pm & 26 & 701 & \pm & 71 & sis \\
\hline GLY & 321 & $\pm \quad 27$ & & 269 & \pm & 11 & 235 & \pm & $11^{1 a}$ & 244 & \pm & 27 & la \\
\hline THR & 177 & \pm 10 & & 136 & \pm & $6^{2}$ & 118 & \pm & $4^{30}$ & 140 & \pm & 10 & $1 x$ \\
\hline HIS & 62 & \pm & 4 & 56 & \pm & 3 & 55 & \pm & 2 & 67 & \pm & 7 & \\
\hline $\mathrm{CIT}$ & 53 & \pm & 5 & 40 & \pm & $3^{1}$ & 48 & \pm & 3 & 22 & \pm & & 362 \\
\hline ALA & 390 & \pm 41 & & 261 & \pm & $12^{2}$ & 306 & \pm & 34 & 415 & \pm & 83 & a \\
\hline TAU & 143 & \pm 17 & & 134 & \pm & 19 & 128 & \pm & 22 & 82 & \pm & 18 & 18 \\
\hline ARG & 88 & \pm 7 & 7 & 78 & \pm & 5 & 90 & \pm & 5 & 83 & \pm & 10 & \\
\hline$\alpha A B$ & 10 & \pm & 2 & 27 & \pm & $7^{1}$ & 13 & \pm & 4 & 19 & \pm & 10 & \\
\hline TYR & 84 & \pm & 6 & 65 & \pm & $4^{1}$ & 61 & \pm & 3 & 75 & \pm & 6 & \\
\hline VAL & 214 & \pm & 7 & 182 & \pm & $7^{2}$ & 156 & \pm & $6^{3 b}$ & 158 & \pm & 13 & 2 \\
\hline MET & 46 & \pm & 2 & 40 & \pm & 2 & 43 & \pm & 2 & 51 & \pm & & 8 \\
\hline ILE & 103 & \pm & 4 & 84 & \pm & $3^{2}$ & 76 & \pm & $3^{3 a}$ & 76 & \pm & 7 & 1 \\
\hline PHE & 70 & \pm & 4 & 60 & \pm & $2^{1}$ & 60 & \pm & $2^{1}$ & 72 & \pm & 8 & \\
\hline TRP & 44 & $\pm \quad 2$ & 2 & 39 & \pm & 3 & 33 & \pm & $1^{3}$ & 36 & \pm & 3 & 1 \\
\hline LEU & 172 & $\pm \quad 10$ & & 135 & \pm & $4^{2}$ & 121 & \pm & $5^{2 .}$ & 124 & \pm & 11 & 2 \\
\hline LYS & 399 & \pm 40 & & 329 & \pm & 13 & 324 & \pm & 16 & 331 & \pm & 35 & \\
\hline BCAA & 489 & \pm 21 & & 401 & \pm & $13^{2}$ & 353 & & 14 & 358 & \pm & 31 & 1 \\
\hline$\alpha A N$ & 3285 & \pm 214 & & 2740 & \pm & $66^{1}$ & 2697 & \pm 1 & $114^{1}$ & 2969 & \pm & 326 & \\
\hline
\end{tabular}

Values are $\mu \mathrm{mol} \mathrm{L}^{-1}$, except urea (mmol $\left.\mathrm{L}{ }^{\prime}\right)$. Mann-Whitney test: versus CON1: ${ }^{1} \mathrm{P}<0.05,{ }^{2} \mathrm{P}<0.01,{ }^{3} \mathrm{P}<0.001$, versus CON2: ${ }^{9} P<0.05,{ }^{\circ} P<0.01,{ }^{c} P<0.001$, versus SHAM: ${ }^{x} P<0.05,{ }^{P} P<0.01,{ }^{x} P<0.001$.

variance $(\mathrm{CV})$ for the PAH determination was $2 \%$. The $\mathrm{CV}$ for the spectrophotometric determinations was well below $4 \%$, the $\mathrm{CV}$ for the amino acids determination was approximately $3 \%$ (e.g. $2 \%$ for glutamine, $3 \%$ for alanine) (20). Inter-assay variation was below $4 \%$.

Tissue processing The frozen tissue specimens were mechanically fragmented with a mortar and pestle, placed in liquid nitrogen. Approximately 0.5 gram of tissue powder was added to a preweighed vial containing $1.5 \mathrm{~mL}$ of ice-cold $5 \%$ SSA. Norvaline was added to a final concentration of $0.5 \mathrm{mM}$ as an internal standard for the amino acids determinations. Tissue weights were determined by reweighing the vial. The tissue fragments were immediately homogenized with an Ultra Turrax (Janke and Kunkel, IKA-Labortechnik, Staufen i. Br., F.R.G.) at $-10^{\circ} \mathrm{C}$ for 30 seconds ( 5 seconds on/off). To remove adherent tissue, the Ultra Turrax was flushed with $0.5 \mathrm{~mL}$ of ice-cold $5 \%$ SSA, which was added to the homogenate. The homogenate was allowed to stand for $5 \mathrm{~min}$ at $-10^{\circ} \mathrm{C}$ for deproteinization and then frozen in liquid nitrogen and stored at $-70^{\circ} \mathrm{C}$.

For determinations, the homogenates were thawed and centrifugated during $5 \mathrm{~min}$ at $4{ }^{\circ} \mathrm{C}$ at $8,500 \mathrm{~g}$. The supernatant was then again centrifugated and divided in portions of $200 \mu \mathrm{L}$. 
Table 2. PDV flow and fluxes.

\begin{tabular}{|c|c|c|c|c|c|c|c|c|c|c|c|c|c|c|}
\hline & & CON1 & $P$ & & CON & & $P$ & & HAM & $F$ & & ENT & & $P$ \\
\hline Flow & 3.0 & \pm 0.8 & & 1.1 & \pm & $0.2^{\prime}$ & & 4.0 & \pm 1. & ' & 1.1 & \pm & 0.2 & $1 x$ \\
\hline Amm & 397 & \pm 89 & & 135 & \pm & 32 & & 493 & \pm 9 & b & 282 & \pm & 70 & - \\
\hline Urea & -2379 & \pm 1744 & & -206 & \pm & 51 & & -552 & \pm 29 & $\phi$ & -396 & \pm & 102 & 14 \\
\hline GLU & 46 & \pm 26 & & 17 & \pm & 7 & & 51 & \pm 1 & b & 12 & \pm & 8 & $y$ \\
\hline ASN & 75 & \pm 29 & & 11 & \pm & 31 & 1 & 18 & \pm 1 & $\phi$ & 0 & \pm & & ${ }^{2} \phi_{\phi}$ \\
\hline SER & 55 & $\pm \quad 47$ & $\phi$ & -8 & \pm & 5 & & .66 & \pm 1 & 2 & -24 & \pm & 7 & $y$ \\
\hline GLN & .700 & \pm 117 & & -213 & \pm & $41^{3}$ & 3 & -901 & \pm 9 & $\mathrm{c}$ & -205 & \pm & 34 & 32 \\
\hline GLY & 233 & \pm 66 & & 45 & \pm & $13^{\prime}$ & 1 & 167 & \pm 3 & b & 23 & \pm & 11 & 12 \\
\hline THA & 57 & \pm 33 & & 3 & \pm & $3^{1}$ & $1 \phi$ & -29 & \pm 1 & 16 & -8 & \pm & 3 & $12 x$ \\
\hline HIS & 24 & $\pm \quad 15$ & & 2 & \pm & 3 & $\phi$ & 2 & \pm & $\phi$ & -2 & \pm & 2 & $1 \phi$ \\
\hline CIT & 112 & \pm 29 & & 26 & \pm & $5^{3}$ & 3 & 112 & \pm 1 & $c$ & 12 & \pm & 4 & $3 a x$ \\
\hline ALA & 679 & \pm 192 & & 178 & \pm & $33^{\prime}$ & ' & 575 & \pm 9 & e & 95 & \pm & 25 & $2 a x$ \\
\hline TAU & 155 & \pm 52 & & 25 & \pm & $14^{\prime}$ & & 80 & \pm 2 & a & 9 & \pm & 6 & ay \\
\hline ARG & 29 & \pm 28 & $\phi$ & 0 & \pm & 4 & $\phi$ & -56 & \pm 1 & * & -9 & \pm & 5 & $y$ \\
\hline TYR & 12 & \pm 14 & $\phi$ & 2 & \pm & 1 & $\phi$ & -11 & \pm & ' & -3 & \pm & 2 & $\phi$ \\
\hline VAL & 38 & \pm 37 & $\phi$ & 2 & \pm & 3 & $\phi$ & -30 & \pm & . & -5 & \pm & 3 & ${ }^{x} \phi$ \\
\hline MET & 12 & \pm 7 & & 2 & \pm & 1 & & -8 & \pm & 12 & -3 & \pm & 2 & $' \phi$ \\
\hline TRP & 2 & \pm & $\phi$ & 0 & \pm & 1 & $\phi$ & -6 & \pm & - & 0 & \pm & & $\phi$ \\
\hline LYS & 166 & $\pm \quad 82$ & & 11 & \pm & $9^{1}$ & $1 \phi$ & -35 & \pm 2 & 2 & -17 & \pm & 13 & $' \phi$ \\
\hline$\alpha A N$ & 1133 & \pm 625 & & 107 & & 56 & & -152 & \pm 16 & 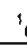 & -128 & & 93 & ${ }^{10} \phi$ \\
\hline
\end{tabular}

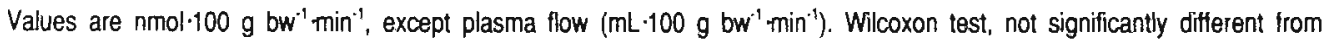
zero: $\phi$. Mann-Whitney test: versus CON1: ${ }^{P}<0.05,{ }^{2} P<0.01,{ }^{3} P<0.001$, versus CON2: $P<0.05$, ${ }^{b} P<0.01$, $P<0.001$, versus SHAM: ${ }^{x} P<0.05,{ }^{y} P<0.01,{ }^{2} P<0.001$. No changes were observed in $\alpha A B A, P H E, L E U, I L E$ and $B C A A$ (not shown).

These were neutralized with $30 \mu \mathrm{L}$ of $2 \mathrm{M} \mathrm{KHCO}_{3}$ to a $\mathrm{pH}$ of 5.0 , after which they were immediately determined (ammonia) or stored (amino acids) at $-70^{\circ} \mathrm{C}$ until determination. For $\% \mathrm{H}_{2} \mathrm{O}$ determinations tissue specimens were thawed to $22^{\circ} \mathrm{C}$ in closed vials (wet weight). The dry weight was determined after freeze drying. The $\% \mathrm{H}_{2} \mathrm{O}$ was calculated as 100 * (wet weight - dry weight)/ wet weight. Total nitrogen of the dry tissue specimens was determined by an elementary analyzer (CHN-O-RAPID, Heraeus, Hanau, F.R.G.).

Calculations The $\alpha$-Amino Nitrogen ( $\alpha$-AN) was calculated as the sum of the amino acids, indicated in Table 1. Branched chain amino acids (BCAA) were calculated as the sum of valine, leucine and isoleucine. Blood flow across liver, portal drained viscera and hindquarter was calculated using the formula introduced by Katz and Bergmann (21), based on the Fick principle for indicator dilution methods:

$$
F_{L P O V, H Q}=\frac{100 \times 1}{\left([P A H]_{L P, V}-(P A H]_{A}\right) \times \text { body weight }(b W)}
$$

where $\mathrm{F}_{\mathrm{L}, \mathrm{PDV}, \mathrm{HQ}}$ is blood flow $\left(\mathrm{mL} \cdot 100 \mathrm{~g}^{-1}\right.$ bw $\left.\mathrm{min}^{-1}\right)$ across liver $(\mathrm{L})$, portal drained viscera (PDV) or hindquarter (HQ), I is the infused PAH $\left(\mu \mathrm{mol}^{-1} \mathrm{~min}^{-1}\right)$, and $[\mathrm{PAH}]_{\mathrm{A}, L, P, V}$ are the concentrations of PAH $\left(\mu \mathrm{mol} \cdot \mathrm{L}^{-1}\right)$ in hepatic $(\mathrm{L})$, portal $(\mathrm{P})$ and inferior caval venous $(\mathrm{V})$, and arterial (A) blood, respectively. Plasma flows were calculated as Flow plasma $_{\text {a }}=$ 
Table 3. Gut tissue concentrations and water content.

\begin{tabular}{|c|c|c|c|c|c|c|c|c|c|}
\hline & & CON1 & $P$ & CON2 & & SHAM & $P$ & ENT & $P$ \\
\hline$\% \mathrm{H}_{2} \mathrm{O}$ & 77.42 & \pm 0.98 & 76.72 & \pm 0.54 & 76.87 & \pm 0.76 & 78.05 & \pm 0.54 & \\
\hline $\mathrm{N}$ & 111.5 & \pm 2.2 & 114.0 & \pm 2.0 & 108.0 & $\pm 1.8^{\circ}$ & 109.5 & \pm 3.5 & \\
\hline ASP & 24.4 & \pm 2.2 & 20.2 & \pm 2.0 & 19.2 & \pm 1.3 & 27.1 & \pm 5.0 & \\
\hline GLU & 125.1 & \pm 6.7 & 126.2 & \pm 11.2 & 124.2 & \pm 6.6 & 155.3 & $\pm 7.1^{2}$ & $x y$ \\
\hline GLN & 28.6 & \pm 2.9 & 33.2 & $\pm \quad 3.9$ & 32.0 & \pm 1.8 & 42.8 & $\pm 2.1^{2}$ & $2 a y$ \\
\hline GLY & 65.5 & \pm 8.8 & 50.1 & \pm 3.5 & 47.9 & $\pm 2.8^{\prime}$ & 63.6 & \pm 5.8 & x \\
\hline THR & 19.2 & \pm 4.8 & 13.2 & \pm 0.9 & 11.2 & \pm 1.0 & 15.6 & $\pm 1.8 \times$ & x \\
\hline HIS & 15.2 & \pm 1.8 & 8.1 & $\pm 1.5^{2}$ & 9.4 & $\pm 1.6^{1}$ & 10.0 & \pm 2.4 & \\
\hline CIT & 8.0 & \pm 1.1 & 6.0 & \pm 0.6 & 7.4 & \pm 0.7 & 7.4 & $\pm 0.5^{\circ}$ & 8 \\
\hline$A\llcorner A$ & 65.2 & \pm 16.4 & 50.7 & \pm 2.6 & 55.2 & \pm 5.6 & 77.4 & $\pm 11.7^{\circ}$ & a \\
\hline TYR & 7.7 & \pm 2.4 & 6.5 & \pm 0.7 & 4.8 & $\pm 0.6^{\circ}$ & 7.6 & \pm 1.3 & \\
\hline$\underline{\text { LYS }}$ & 18.6 & $\pm \quad 5.7$ & 14.8 & $\pm \quad 1.4$ & 15.2 & \pm 1.5 & 18.9 & \pm 1.8 & $\mathrm{ax}$ \\
\hline
\end{tabular}

Values are $\mu \mathrm{mol} \mathrm{kg} \mathrm{N}^{-1}$, except $\% \mathrm{H}_{2} \mathrm{O}$ and $\mathrm{N}$ ( $g \mathrm{~kg}$ dry weight $\mathrm{t}^{-1}$ ). Mann-Whitney test: versus CON1: ${ }^{\mathrm{P}} \mathrm{P}<0.05,{ }^{2} \mathrm{P}<0.01$, ${ }^{3} P<0.001$, versus CON2: ${ }^{a} P<0.05,{ }^{D} P<0.01,{ }^{c} P<0.001$, versus SHAM: ${ }^{x} P<0.05,{ }^{r} P<0.01,{ }^{2} P<0.001$. No changes were observed in Amm, aAAA, ASN, SER, TAU, AHG, $\alpha A B A, V A L, M E T$, ILE, PHE, TRP, LEU, BCAA, $\alpha A N$ (not shown).

( 1 - hematocrit) $\cdot$ Flow $_{\text {blood }}$. PDV flux was calculated by multiplication of the portal-arterial concentration difference with the PDV plasma flow. Splanchnic flux was calculated by multiplication of the hepatic-arterial difference and the liver plasma flow. Liver flux was calculated by subtracting the PDV flux from the splanchnic flux. Hindquarter flux was calculated as hindquarter plasma flow times caval vein-arterial concentration difference. Flux is expressed per $100 \mathrm{~g} \mathrm{bw} \cdot \mathrm{min}^{-1}$. A positive figure means net efflux, negative values reflect net uptake.

Statistical analysis Results are presented as means \pm SEM. The level of significance was set at $\mathrm{P}<0.05$. For statistical analysis (22) the Mann-Whitney non-parametric test was used for comparison of differences between the CON1, CON2, SHAM and ENT group. Significance from zero was tested by the Wilcoxon test.

\section{RESULTS}

Arterial values (Table 1, Figure 1-3) A 40h starvation period (CON2), compared to an overnight fast (CON1) did not change arterial glutamine, although a significant decrease of the arterial concentration of most amino acids, including glutamate, alanine and BCAA was observed. However, 24h after enterectomy, compared to SHAM operation, a significant increase of glutamine was observed, concomitant with increased ammonia, urea and threonine and a $50 \%$ decrease of citrulline. SHAM operation, compared to CON2, caused a decrease of glycine, threonine and BCAA.

Plasma flow (Table 2, 4 and 7) As expected, 80\% enterectomy significantly lowered PDV plasma flow in comparison to the SHAM group; liver and hindquarter flow did not change significantly. After 40h of starvation (CON2), PDV blood flow decreased, but liver and hindquarter flow did not change. SHAM operation induced an increase in PDV blood 
Table 4. Liver flow and fluxes.

\begin{tabular}{|c|c|c|c|c|c|c|c|c|c|c|c|}
\hline & & CON1 & $p$ & & CON2 & & SHAM & $P$ & & ENT & $P$ \\
\hline Flow & 3.6 & \pm 0.8 & & 1.9 & \pm 0.4 & 5.2 & \pm 1.9 & & 2.4 & \pm 0.5 & \\
\hline Amm & -520 & \pm 100 & & -203 & $\pm 37^{2}$ & -497 & \pm 150 & & -357 & \pm 71 & \\
\hline Urea & 3359 & \pm 1811 & & 476 & $\pm 170^{2}$ & 2258 & \pm 830 & & 743 & \pm 341 & \\
\hline GLU & 104 & \pm 47 & & 17 & $\pm 15^{\prime} \phi$ & -13 & \pm 30 & $' \phi$ & 7 & \pm 19 & $' \phi$ \\
\hline ASN & -138 & \pm 45 & & -52 & \pm 5 & -126 & \pm 21 & $\circ$ & .74 & \pm 18 & ' \\
\hline SER & -322 & \pm 84 & & -159 & \pm 13 & .328 & \pm 56 & & .204 & $\pm \quad 41$ & \\
\hline GLN & 11 & \pm 73 & $\phi$ & -162 & $\pm 51^{1}$ & -245 & \pm 125 & & -479 & $\pm 119^{2}$ & $a$ \\
\hline GLY & -434 & \pm 125 & & -191 & $\pm \quad 21$ & -583 & \pm 82 & & -283 & \pm 60 & $\mathbf{x}$ \\
\hline THR & .154 & \pm 63 & & .70 & \pm 8 & -160 & \pm 28 & & -116 & $\pm \quad 28$ & \\
\hline $\mathrm{CIT}$ & -99 & \pm 45 & & -7 & $\pm \quad 4^{\prime} \phi$ & -63 & \pm 18 & $\circ$ & -8 & $\pm \quad 4^{\prime}$ & y \\
\hline ALA & -1247 & \pm 329 & & -461 & $\pm 42^{2}$ & -1186 & \pm 189 & ' & -473 & $\pm 101^{\prime}$ & $1 x$ \\
\hline TAU & -236 & \pm 94 & & -61 & $\pm \quad 12$ & -128 & \pm 17 & $b$ & -67 & $\pm 35^{\prime}$ & $r$ \\
\hline VAL & 56 & \pm 33 & $\phi$ & 16 & $\pm 8^{1}$ & 33 & \pm 19 & & 25 & \pm 15 & $\phi$ \\
\hline MET & -26 & 9 & & -14 & \pm & -35 & \pm 6 & & -22 & \pm 6 & \\
\hline PHE & -46 & $\pm \quad 22$ & & -17 & \pm & -49 & \pm 11 & ' & -31 & \pm 12 & ' \\
\hline TRP & 16 & \pm 6 & & 5 & $2^{1}$ & 12 & \pm 6 & $\phi$ & 1 & \pm 2 & ' $\phi$ \\
\hline LYS & -415 & \pm 152 & & -153 & \pm 18 & -336 & \pm 56 & & -216 & \pm 66 & \\
\hline$\alpha \mathrm{AN}$ & -3060 & \pm 1076 & & -1380 & \pm 156 & -3368 & \pm 505 & 4 & -2046 & \pm 514 & \\
\hline
\end{tabular}

Values are nmol.100 g bw $\mathrm{m}^{-1} \mathrm{~min}^{-1}$, except plasma flow $\left(\mathrm{mL} \cdot 100 \mathrm{~g} \mathrm{bw}^{-1} \mathrm{~min}^{-1}\right)$. Wilcoxon test, not significantly different from zero: $\phi$. Mann-Whitney test: versus CON1: $P<0.05,{ }^{2} P<0.01,{ }^{3} P<0.001$, versus CON2: $P<0.05,{ }^{D} P<0.01,{ }^{C} P<0.001$, versus SHAM: ${ }^{x} P<0.05,{ }^{y} P<0.01,{ }^{x} P<0.001$. No changes were observed in HIS, ARG, $\alpha A B A, T Y R, I L E, L E U$ and BCAA (not shown).

flow.

PDV Fluxes (Table 2) and intra-cellular gut concentrations (Table 3) After $80 \%$ enterectomy, the PDV uptake of glutamine (Figure 1) was decreased by $80 \%$ in comparison to the SHAM group, while the ammonia efflux was decreased by $40 \%$. Also, the fluxes of most other amino acids were lower than in the SHAM group. The intra-cellular gut concentrations of glutamine (Figure 1), glutamate (Figure 2), glycine, threonine and lysine were increased after enterectomy.

SHAM operation, compared to CON2 induced an increased net PDV consumption of glutamine and several other amino acids and an increased net PDV efflux of ammonia, glutamate (Figure 2), glycine, citrulline, alanine (Figure 3) and taurine. The intra-cellular gut concentrations of tyrosine and nitrogen were lower in the SHAM group.

40h Starvation (CON2) in comparison to overnight fast (CON1) induced a decreased PDV ammonia release and glutamine uptake (Figure 1). A decreased release of most of the other amino acids was observed without a change in their intra-cellular gut concentrations, except HIS, which was decreased in the CON2 group.

Fluxes and intracellular concentrations: liver and splanchnic region (Table 4-6) In enterectomized rats glutamine uptake by the liver was not significantly different (Figure 1), compared to SHAM operated animals. Asparagine, glycine, citrulline, alanine (Figure 3) and taurine uptake were lower in the enterectomy group. The intra-cellular liver concentration of aspartate and methionine was higher in the enterectomy group. 


\section{Chapter 4}

Table 5. Liver tissue concentrations.

\begin{tabular}{|c|c|c|c|c|c|c|c|c|c|}
\hline & & CON1 & $p$ & CON2 & & SHAM & & ENT & $P$ \\
\hline$\% \mathrm{H}_{2} \mathrm{O}$ & 68.69 & \pm 0.35 & 68.71 & \pm 0.47 & 69.47 & \pm 0.48 & 70.59 & \pm 0.39 & $\not b$ \\
\hline$N$ & 124.2 & \pm 0.7 & 123.9 & \pm 1.2 & 123.2 & \pm 1.1 & 124.3 & \pm 1.1 & \\
\hline ASP & 29.1 & \pm 1.8 & 23.3 & \pm 2.2 & 22.2 & $\pm 1.2^{2}$ & 27.1 & \pm 1.1 & x \\
\hline GLU & 81.1 & \pm 9.2 & 82.6 & \pm 7.4 & 87.7 & \pm 9.2 & 97.8 & \pm 12.3 & \\
\hline GLN & 97.7 & \pm 9.3 & 95.7 & \pm 5.4 & 99.6 & \pm 8.1 & 89.8 & \pm 5.0 & \\
\hline GLY & 68.5 & \pm 4.4 & 64.9 & \pm 4.3 & 63.7 & \pm 4.1 & 56.9 & \pm 4.4 & 1 \\
\hline THR & 10.4 & \pm 0.9 & 8.2 & \pm 0.6 & 9.9 & \pm 0.8 & 12.4 & \pm 1.0 & b \\
\hline CIT & 1.1 & \pm 0.2 & 0.9 & \pm 0.1 & 0.7 & $\pm 0.1^{1}$ & 0.5 & \pm 0.1 & 2 \\
\hline ALA & 28.0 & \pm 4.1 & 30.5 & \pm 6.3 & 52.7 & \pm 15.2 & 84.9 & \pm 11.2 & 30 \\
\hline ARG & 0.7 & \pm 0.1 & 1.3 & \pm 0.4 & 0.7 & \pm 0.1 & 0.7 & \pm 0.2 & a \\
\hline$\alpha A B A$ & 0.8 & \pm 0.1 & 0.5 & $\pm 0.1^{1}$ & 0.9 & \pm 0.2 & 1.0 & \pm 0.1 & o \\
\hline VAL & 10.8 & \pm 0.6 & 7.7 & $\pm 0.6^{2}$ & 6.5 & $\pm 0.7^{2}$ & 7.0 & \pm 0.7 & 2 \\
\hline MET & 1.6 & \pm 0.2 & 1.1 & \pm 0.2 & 0.9 & $\pm 0.1^{2}$ & 1.5 & \pm 0.2 & $y$ \\
\hline ILE & 4.2 & \pm 0.5 & 3.5 & \pm 0.4 & 3.1 & $\pm 0.3^{\prime}$ & 3.2 & \pm 0.3 & \\
\hline TRP & 0.5 & \pm 0.0 & 0.3 & $\pm 0.1^{1}$ & 0.4 & \pm 0.0 & 0.5 & \pm 0.1 & \\
\hline LEU & 7.4 & $\pm \quad 0.7$ & 5.9 & \pm 0.6 & 5.1 & \pm 0.5 & 5.4 & \pm 0.5 & 1 \\
\hline LYS & 25.3 & $\pm \quad 3.7$ & 16.7 & $\pm 1.4^{1}$ & 14.7 & $\pm 1.7^{2}$ & 14.7 & \pm 1.0 & 2 \\
\hline$B C A A$ & 22.5 & \pm 1.7 & 17.1 & $\pm 1.6^{1}$ & 14.7 & $\pm 1.5^{2}$ & 15.6 & \pm 1.6 & $\stackrel{1}{1}$ \\
\hline
\end{tabular}

Values are $\mu \mathrm{mol} \mathrm{kg} \mathrm{N}^{1}{ }^{-1}$, except $\% \mathrm{H}_{2} \mathrm{O}$ and $\mathrm{N}$ ( $\mathrm{g} \mathrm{kg}$ dry weight'). Mann-Whitney lest: versus CON1: ${ }^{\prime} \mathrm{P}<0.05,{ }^{2} \mathrm{P}<0.01$, ${ }^{3} \mathrm{P}<0.001$, versus CON2: ${ }^{\mathrm{P}} \mathrm{P}<0.05$, ${ }^{\mathrm{P}} \mathrm{P}<0.01,{ }^{c} \mathrm{P}<0.001$, versus $S H A M:{ }^{x} \mathrm{P}<0.05,{ }^{\mathrm{y} P}<0.01,{ }^{\mathrm{P}} \mathrm{P}<0.001$. No changes were observed in Amm, aAAA, ASN, SER, HIS, TAU, TYR, PHE, $\alpha A N$ (not shown).

Table 6. Splanchnic flow and fluxes.

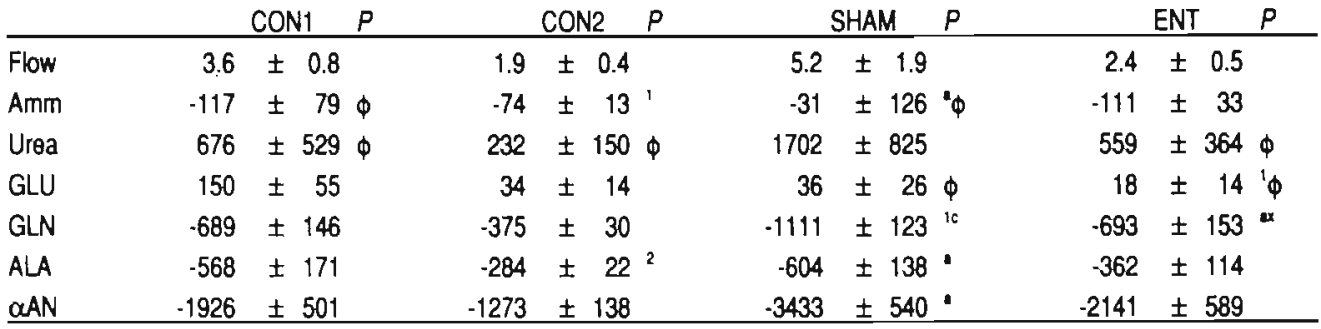

Values are nmol.100 $\mathrm{g} \mathrm{bw}^{-1} \mathrm{~min}^{-1}$, except plasma flow $\left(\mathrm{mL} \cdot 100 \mathrm{~g} \mathrm{bw}^{-1} \mathrm{~min}^{-1}\right)$. Wilcoxon test, not significantly different from zero: $\phi$. Mann-Whitney test: versus CON1: ${ }^{1}<<0.05,{ }^{2} P<0.01,{ }^{3} P<0.001$, versus CON2: ${ }^{a}<<0.05$, ${ }^{D} P<0.01,{ }^{c} P<0.001$, versus SHAM: ${ }^{x} P<0.05,{ }^{\prime} P<0.01,{ }^{2} P<0.001$.

The altered glutamine flux across the PDV and the unchanged flux across liver after enterectomy resulted in a total splanchnic glutamine uptake that was lower than in SHAM operated rats. 
Table 7. Hindquarter flow and fluxes.

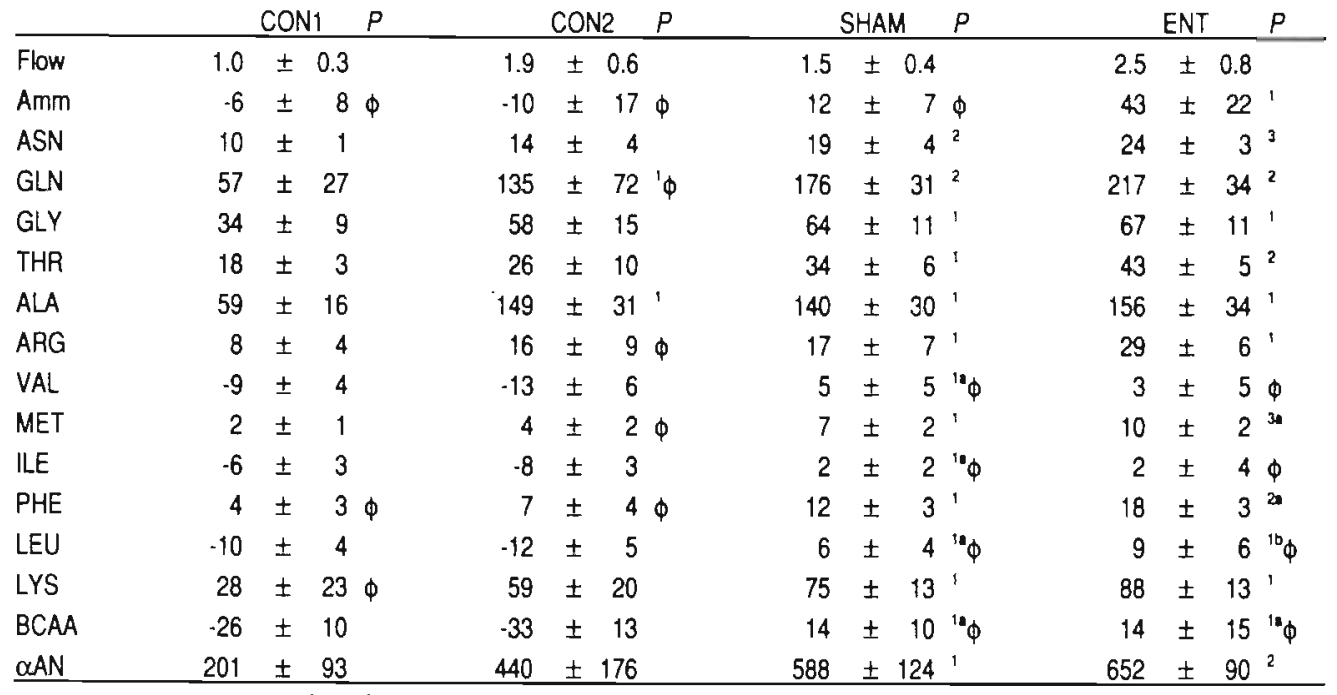

Values are $\mathrm{nmol} \cdot 100 \mathrm{~g} \mathrm{bw}^{-1} \cdot \mathrm{min}^{-1}$, except plasma flow $\left(\mathrm{mL} \cdot 100 \mathrm{~g} \mathrm{bw}^{-1} \mathrm{~min}^{-1}\right)$. Wilcoxon test, not significantly different from zero $\phi$. Mann-Whitney test: versus CON1: ' $P<0.05,{ }^{2} P<0.01,{ }^{3} P<0.001$, versus CON2: ${ }^{8} P<0.05,{ }^{D} P<0.01,{ }^{6} P<0.001$, versus SHAM: ${ }^{x} P<0.05,{ }^{y} P<0.01,{ }^{2} P<0.001$. No changes were observed in urea, GLU, SEF, HIS, CIT, TAU, $\alpha A B A, T Y A$, TAP (not shown).

Table 8. Muscle tissue concentrations.

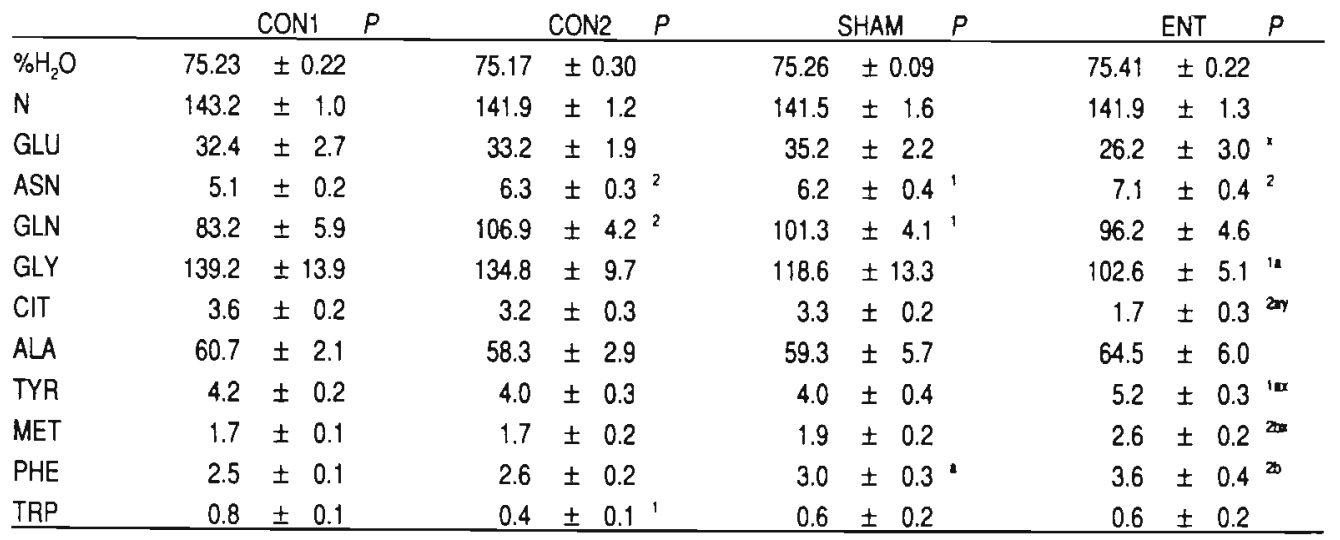

Values are $\mu$ mol $k g \mathrm{~N}^{-1}$, except $\% \mathrm{H}_{2} \mathrm{O}$ and $\mathrm{N}\left(\mathrm{g} \mathrm{kg}\right.$ dry weight ${ }^{1}$ ). Mann-Whitney test: versus CON1: $P<0.05,{ }^{2} \mathrm{P}<0.01$, ${ }^{3} P<0.001$, versus CON2: ${ }^{a} P<0.05,{ }^{b} P<0.01,{ }^{c} P<0.001$, versus SHAM: ${ }^{x} P<0.05,{ }^{\prime} P<0.01,{ }^{x} P<0.001$. No changes were observed in Amm, ASP, aAAA, SER, THR, HIS, TAU, ARG, CAR (Carnosine), વABA, VAL, LLE, LEU, LYS, BCAA, $\alpha A N$ (not shown). 
The SHAM operation did not change glutamine uptake by the liver, compared to unoperated controls (CON2). However, the uptake of most other amino acids and ammonia was increased. Liver urea production was not significantly different. No differences were observed in tissue concentrations.

$40 \mathrm{~h}$ Starvation in comparison to ovemight fasting induced a decreased hepatic urea, glutamate (Figure 2), valine and tryptophan efflux, decreased ammonia, citrulline and alanine (Figure 3) uptake and a higher glutamine uptake (Figure 1), without major changes in the intra-cellular liver concentrations, except for decreases in $\alpha \mathrm{ABA}$, valine, tryptophan, lysine and BCAA.

Hindquarter fluxes and intracellular muscle concentrations (Table 7,8) Although after $80 \%$ enterectomy splanchnic glutamine uptake (Figure 1) was less than in SHAM operated animals, hindquarter glutamine release was not significantly different between the ENT and SHAM group. In fact, no differences in hindquarter fluxes were observed between the SHAM and ENT group. However, after enterectomy, muscle intra-cellular concentrations were different, especially the decrease of intra-cellular glutamate (Figure 2) and citrulline. Also, a slight increase of the tyrosine and methionine concentration was observed.

After $40 \mathrm{~h}$ of starvation, net glutamine and alanine (Figure 3) efflux was increased, being more than $50 \%$ of total $\alpha$-amino acid release. Intra-cellular glutamine was higher, but alanine was unchanged. Although both SHAM operation and enterectomy caused BCAA uptake across the hindquarter to decrease, intra-cellular concentrations remained unchanged.

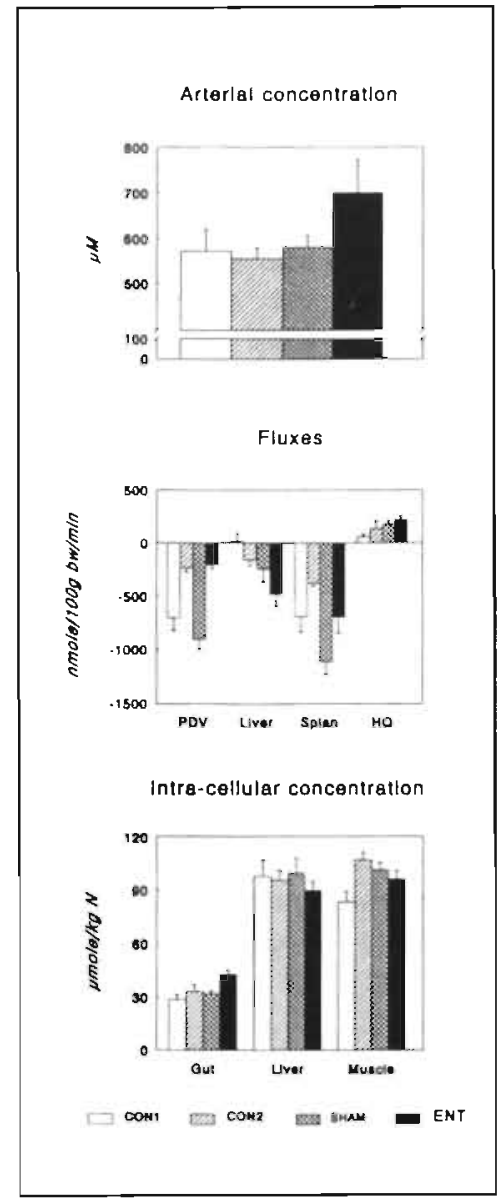

Figure 1. Glutamine: the arterial concentration (upper panel: $\mu \mathrm{M}$ ), fluxes (middle panel: $\mathrm{nmol} \cdot 100 \mathrm{~g} \mathrm{bw}^{-1} \mathrm{~min}^{-1}$ ) and intra-cellular concentration (lower panel: $\mu \mathrm{mo} / \mathrm{kg} \mathrm{N}^{-1}$ ).

\section{DISCUSSION}

To study the relationship between gut and liver glutamine consumption and peripheral (muscle) glutamine production, we studied the effect of subtotal small bowel resection in the rat. This model could give insight whether the proposed hypothesis that "consumption of glutamine by the splanchnic tissues is an important determinant for its production in muscle" is valid.

Enterectomy in rats (23) (this study) and dogs (15) induced a decrease of gut glutamine extraction. In humans, small intestine resection induced a $20 \%$ decrease in whole body glutamine utilization (16). To facilitate the detection of any potential effect of decreased splanchnic glutamine consumption on muscle glutamine release, we attempted to enhance the latter, prior to enterectomy, by superimposing surgery on a starvation period. This contrasts 
with previous acute studies in rats (23) and dogs (15, 24).

After enterectomy, total splanchnic glutamine uptake decreased only by $38 \%$, although gut glutamine uptake decreased by $77 \%$. Apparently, the liver partially compensated the decreased gut glutamine uptake. Also, we observed increased arterial glutamine concentrations after $80 \%$ enterectomy, suggesting that subtotal small bowel resection in the rat has substantial effects on body glutamine metabolism. Although muscle is an important organ in body glutamine metabolism, release of glutamine by the hindquarter was not significantly different between SHAM and enterectomy animals. The increase in the arterial glutamine levels, the lower uptake by the remaining gut and the apparent compensatory increase of uptake by the liver, suggest that glutamine release by the peripheral tissues is relatively independent of splanchnic uptake, but probably more depending upon the magnitude of the trauma. Similarly, the increased release of glutamine by the hindquarter after starvation and the SHAM operation did not coincide with an increased glutamine uptake by the splanchnic tissues. Therefore, our results do not support the hypothesis that splanchnic glutamine uptake is an important regulator of peripheral (muscle) glutamine release $(8,15)$.

Enterectomy, in comparison to SHAM operation diminished the net PDV flux of most amino acids, probably related to the reduced tissue mass. However, the decrease of the glutamine flux was much less than of the flux of for example glycine, citrulline, alanine and valine. It was found that gut glutamine extraction was lower, immediately after $60 \%$ enterectomy in rats (23). Also, 6 hours after $60 \%$ enterectomy in dogs, glutamine uptake was found to be only $6 \%$ of the control uptake. However, one week later, glutamine extraction was higher in enterectomized dogs than in controls. This could mean that glutamine demand and degradation per gram tissue in the remaining gut after enterectomy is increased after $24 \mathrm{~h}$ and further on.

The $73 \%$ decrease of PDV plasma flow partly explains the $54 \%$ decrease of liver plasma flow. Plasma flow through the hepatic artery (liver flow minus portal flow) was unchanged in agreement with studies in dogs $(15,24)$, implying that the (relative) amount of substrate delivery to the liver changes from mainly portal to arterial.

After enterectomy, lower quantities of most of the amino acids were taken up by the liver. However, glutamine uptake did not decrease, in contrast to studies in dogs (15) in which $6 \mathrm{~h}$ after enterectomy, liver glutamine uptake even changed to release, a finding 
possibly related to species differences (1). The absence of a significant change in hepatic glutamine flux, combined with the decreased uptake of most other amino acids caused that the relative contribution of glutamine to total amino acid uptake was increased and therefore its potential contribution to hepatic urea release. It appears that in this situation glutamine uptake is related to the increased arterial glutamine concentration, compatible with the fact that hepatic glutamine uptake is concentration dependent and not yet saturated at these arterial concentrations (12). The altered substrate delivery could also explain the increased intra-cellular aspartate concentration, an intermediate product of liver glutamine breakdown and urea synthesis.

Although splanchnic urea production and ammonia uptake did not significantly differ between the ENT and SHAM group, both arterial ammonia and urea concentrations were higher during enterectomy. As hindquarter ammonia uptake remained unchanged, the arterial concentration changes are possibly caused by metabolic changes in other organs, presumably the kidney (15). Also, when calculating the glutamine production by muscle (approximately 2.7 times HQ flux (25)) and the splanchnic uptake, a discrepancy exists, especially in the SHAM and CON1 group. Possibly, other organs such as kidney or lung also play a role in the altered glutamine metabolism in the groups, but this can not be deduced from the present experiments.

Of the hindquarter fluxes measured, no changes were observed between the SHAM and enterectomy group. However, particularly the intra-cellular muscle glutamate concentration was lower. Glutamate is an important substrate for muscle glutamine metabolism as glutamine production in muscle requires glutamate. The unchanged muscle glutamine production after enterectomy in relation to the decreased glutamate concentration could point to a role for glutamate as the driving force for muscle glutamine synthesis or, alternatively, that glutamine synthetase is activated to keep intracellular glutamine levels constant. This observation suggests that enterectomy induces changes in muscle metabolism.

No changes were observed in arterial glutamine concentrations after $40 \mathrm{~h}$ starvation compared to overnight fast, although decreased arterial concentrations were observed for most other amino acids. Maintenance of the arterial glutamine concentration apparently is important during starvation.

During starvation, PDV glutamine uptake was decreased by $70 \%$, while in this situation liver glutamine uptake increased. Glutamine consumption during starvation switched from gut to liver, which is probably related to decreased gut metabolism, induced by the starva- 
tion. This altered inter-organ nitrogen transport did not induce major intra-cellular concentration changes in gut and liver. Both muscle release and intra-cellular concentration of glutamine were increased during starvation, indicating increased muscle amino acid deamination, glutamine synthesis (26) and decreased net protein synthesis (27). Both PDV efflux and liver uptake of alanine were decreased, causing total net splanchnic alanine uptake to decrease. However, as muscle alanine efflux increased and arterial concentration decreased, alanine must be taken up by other organs such as kidney (15).

In conclusion, this study shows that despite decreased splanchnic glutamine uptake after $80 \%$ enterectomy in the rat, muscle glutamine production is unchanged. This casts doubt on the hypothesis that diminished splanchnic glutamine uptake can reduce muscle glutamine production.

\section{REFERENCES}

1. Souba WW, Smith RJ, Wilmore DW. Glutamine metabolism by the intestinal tract. JPEN 1985;9:608-617.

2. Johnson DJ, Colpoys M, Smith RJ, Jiang Z, Kapadia CR, Wilmore DW. Branched chain amino acid uptake and muscle free amino acid concentrations predict postoperative muscle nitrogen balance. Ann Surg 1986;204:513-523.

3. Ardawi MSM. Skeletal muscle glutamine production in thermally injured rats. Clin Sci 1988;74:165-172.

4. Albina JE, Shearer J, Mastrofrancesco B, Caldwell MD. Amino acid metabolism after lambdacarrageenan injury to rat skeletal muscle. Am J Physiol 1986;250:E24-E30.

5. O'Connor JA, Scott RL, Mellick PW, Caldwell MD. Perfused rat hindlimb wound model: lambda carrageenan induced. Am J Physiol 1982;242:R570-R576.

6. Rosenblatt S, Clowes GHA, George BC. Hirsch E, Lindberg B. Exchange of amino acids by muscle and liver in sepsis. Arch Surg 1983;1 18:167-175.

7. Finley RJ, Inculet RI, Pace R, Holloday R, Rose C, Duff JH, Groves AC, Woolf LL. Major operative trauma increases peripheral amino acid release during the steady-state infusion of total parenteral nutrition in man. Surgery 1986;99:491-499.

8. Fong Y, Tracey KJ, Hesse DG, Albert JD, Barie PS, Lowry SF. Influence of enterectomy on peripheral tissue glutamine efflux in critically ill patients. Surgery 1990;107:321-326.

9. Souba WW, Wilmore DW. Postoperative alteration of arteriovenous exchange of amino acids across the gastrointestinal tract. Surgery 1983;94:342-350.

10. Goldstein SA, Elwyn DH. The effects of injury and sepsis on fuel utilization. Ann Rev Nutr 1989;9:445-473.

11. Souba WW, Klimberg VS, Plumley DA, Salloum RM, Flynn TC, Bland KI, Copeland EM. The role of glutamine in maintaining a healthy gut and supporting the metabolic response to injury and infection. $J$ Surg Res 1990;48:383-391.

12. Windmueller HG. Glutamine utilization by the small intestine. Adv Enzyme 1982;53:210-237.

13. Lee VM, Wolfe BM, Hansen RJ, Clifford AJ. Postsurgical muscle protein tumover in perfused hindquarters of the rat. JPEN $1988 ; 12: 452-456$.

14. Heindorff $H$, Vilstrup $H$, Bucher $D$, Billebolle $P$, Thygesen $V$. Increased hepatic amino nitrogen conversion after elective cholecystectomy in man. Clin Sci 1988;74:539-545.

15. Souba WW, Roughneen PT, Goldwater DL, Williams JC, Rowlands RJ. Postoperative alterations in interorgan glutamine exchange in enterectomized dogs. J Surg Res 1987;42:117-125.

16. Darmaun D, Messing B, Just B, Rongier M, Desjeux JF. Glutamine metabolism after small intestinal resection in humans. Metabolism 1991;40:42-44.

17. Wollenberger A, Ristau O, Schoffa G. Eine einfache Technik der extrem schnellen Abkühlung größerer Gewebestücke. Pflügers Archiv 1960;270:399-412.

18. Neeley WE, Philipson J. Automated enzymatic method for deternining ammonia in plasma, with 14day reagent stability. Clin Chem 1988;34:1868-1869. 
19. Brun C. A rapid method for the determination of para-aminohippuric acid in kidney function tests. J Lab Clin Med 1951;37:955-958.

20. van Eijk HMH, van der Heijden MAH, van Berlo CLH, Soeters PB. Fully automated liquidchromatographic determination of amino acids. Clin Chem 1988;34:2510-2513.

21. Katz ML, Bergman EN. Simultaneous measurements of hepatic and portal venous blood flow in the sheep and dog. Am J Physiol 1969;216:946-952.

22. Norusis MJ ed.: SPSS/PC+ V3.1 BASE MANUAL for the IBM PC/XT/AT and PS/2. SPSS Inc, Chicago, USA, 1989.

23. Klimberg VS, Souba WW, Salloum RM, Holley DT, Hautamaki RD, Dolson DJ, Copeland EM. Intestinal glutamine metabolism after massive small bowel resection. Am J Surg 1990;159:27-33.

24. Souba WW, Roughneen PT, Goldwater DL, Reed R, Rowlands BJ. Effects of enterectomy on postoperative visceral organ glucose exchange. JPEN 1989;13:128-131.

25. Schröck H, Goldstein L. Interorgan relationships for glutamine metabolism in normal and acidotic rats. Am J Physiol 1981;240:E519-E525.

26. Arola LI, Palou A, Remesar X. Alemany M. Glutamine synthetase activity in the organs of fed and 24$h$ fasted rats. Horm Metab Res 1981; 13:199-202.

27. Jepson MM, Pell JM, Bates PC, Millward DJ. The effects of endotoxemia on protein metabolism in skeletal muscle and liver of fed and fasted rats. Biochem J 1986;235:329-336. 


\section{Chapter 5}

\section{Gut and muscle during acute liver failure ${ }^{1}$}

Published as: C.H.C. Dejong, M.T. Kampman, N.E.P. Deutz, P.B. Soeters. Altered glutamine metabolism in rat portal drained viscera and hindquarter during hyperammonemia. Gastroenterology 1992;102:936-948.

\section{ABSTRACT}

In normal rats, muscle is the major glutamine releasing and gut the major glutamine consuming organ. It has been suggested that enhanced muscle ammonia detoxification and gut ammonia production occurs during liver insufficiency-induced hyperammonemia. Therefore, ammonia and amino acids fluxes across portal drained viscera and hindquarter, and muscle concentrations were measured in portacaval shunted and acute liver ischemia rats.

Arterial ammonia and most amino acids were increased after portacaval shunting and increased progressively during liver ischemia, but net hindquarter ammonia uptake was not observed. Net hindquarter glutamine efflux was increased during portacaval shunting, but it decreased during liver ischemia, while muscle glutamine concentrations increased. The comparable net portal drained viscera glutamine uptake in normal and portacaval shunted rats changed during liver ischemia from net uptake to release, coinciding with release of most other amino acids.

These results cast doubt on the ammonia detoxifying role of muscle during acute liver ischemia-induced hyperammonemia in the rat. The portal drained viscera glutamine release during severe hyperammonemia could be due to intestinal damage.

\section{INTRODUCTION}

Recent hypotheses concerning the etiology of hepatic encephalopathy (HE) have again focussed on hyperammonemia as one of the central pathogenic factors (1-5). A role for muscle in ammonia detoxification has been postulated in this context $(3,6-8)$, because

\footnotetext{
'This chapter is an exact copy of the original publication and consequently does not contain all data from the experiments. Additional data are available on request.
} 
muscle has been shown to take up ammonia under certain conditions (3). Non-hepatic tissues can detoxify ammonia by amidation of glutamate to glutamine (9), a reaction which, in the physiological situation, predominantly takes place in skeletal muscle (10). Although it is generally assumed that muscle is the main glutamine releasing organ in the normal situation (11), the in vivo quantitative role of muscle in ammonia detoxification during liver insufficiency-induced hyperammonemia is unclear $(6,7,12,13)$.

An important glutamine consuming organ in the physiological situation is the gut $(11,13)$. Intestine participates in glutamine and ammonia metabolism in two ways. First, small bowel mucosa produces ammonia by converting blood-borne as well as luminal glutamine to ammonia and glutamate (14-19), the latter being transaminated to alanine and to a lesser degree to other amino acids and organic acids $(13,18)$. Second, colonic bacteria produce ammonia by splitting urea and possibly amino acids (14-16). The ammonia and amino acids are released into the portal blood and transported to the liver (13). Subsequently, ammonia and alanine nitrogen enter the urea cycle, whereas the alanine carbon skeleton is utilized in hepatic gluconeogenesis (20). Thus, in the normal situation ammonia released into the portal vein is to a large extent removed by hepatic urea synthesis (20). Impaired liver function leads to impaired urea synthesis capacity and consequently causes a derangement of the above described metabolic pathways $(20,21)$. Little is known about alternative means of ammonia detoxification in this situation, but enhanced glutamine formation by nonhepatic organs (mainly muscle) has been suggested $(3,6,22,23)$.

To establish the in vivo role of the intestine and muscle as well as their interaction in ammonia and glutamine metabolism during liver insufficiency-induced hyperammonemia in the rat, fluxes of ammonia and amino acids were measured over the portal drained viscera (mainly intestine) and the hindquarter (mainly muscle). The experiments do not confirm the hypothesis that enhanced muscle ammonia detoxification occurs during severely compromised liver function with hyperammonemia. Under these conditions ammonia is released in increased quantities from the portal drained viscera and portal drained viscera glutamine uptake reverses to net release.

\section{MATERIALS AND METHODS}

Experimental Animals All experiments were performed in male SPF Wistar rats ( $\mathrm{n}=63$, 250-350 g, random bred Wistar/CPB:WU/Bor, Winkelmann, Borchen, F.R.G.), housed under standard conditions (not reallocated for at least one week, 12 hour light-dark cycle (light 7:30 AM - 7:30 PM)), and fed standard lab chow and water ad libitum. Drinking water was acidified to $\mathrm{pH}=3$ to prevent bacterial or algae growth (routine procedure in our institute). The project was approved by the local animal ethical committee.

Experimental groups Three major groups were formed (Table 1): One group included normal control rats, without previous surgery. A second group included rats in which a portacaval shunt (PCS) was constructed. These rats either had no operation until sampling the next day at $t=0$ hours ( $P C S O$ ) or underwent a sham operation the next day at $t=0$ hours, mimicking acute liver ischemia (ALI) induction, but omitting the hepatic artery ligation. In these rats, blood was sampled 2, 4 or 6 hours after the sham operation (PCS2, PCS4, PCS6). The third group included rats in which the hepatic artery was ligated 1 day after PCS to induce ALI at $\mathrm{t}=0$ hours. In these rats blood was sampled 2, 4 or 6 hours after ALI induction 
Table 1. Experimental groups.

\begin{tabular}{lcll} 
Group & $n$ & day 0 & day 1 \\
\hline NORM & 11 & no surgery & sampling at to \\
PCS 0 & 7 & PCS & sampling at to \\
PCS 2,4,6 & $7,7,8$ & PCS & SHAM at t0, sampling 2, 4 \\
& & & or 6 hours later. \\
ALI 2,4,6 & $9,8,6$ & PCS & ALI at 10, sampling 2, 4 or \\
& & & 6 hours later. \\
\hline
\end{tabular}

PCS: PortaCaval Shunting, ALI: Acute Liver Ischemia, SHAM: SHAM operation, for details cf. 'Surgical procedures'.

\section{(ALI2, ALI4, ALI6).}

Before PCS the animals were randomly assigned to one of the resulting eight groups (Table 1), using the random function of a computer program (Supercalc 5B, Computer Associates International Inc., San Jose, CA, USA). The surgeon was unaware of the grouping. The time of induction ( $\mathrm{t}=0$ hours) of ALI or the corresponding sham operation was between 8:00 and 10:00 AM.

Surgical procedures All operations were performed at room temperature under ether anesthesia by the same surgeon (CD). Heated operating boards and, if necessary, heating lamps were used to maintain body temperature, monitored rectally using an electronic temperature probe. PCS was performed applying the button technique as described by Funovics (24), using polyethylene buttons (ID $0.17 \mathrm{~cm}, O . D 0.23 \mathrm{~cm}$ ). Before PCS the animals were weighed, the skin of abdomen, right hindlimb, and neck was shaved, and temperature was recorded. After completion of the shunt, the hepatic artery was marked with a silk thread to facilitate induction of ALI. The mean operation time for PCS was 25 minutes, with a portal vein clamping time of about 8 minutes.

Sixteen hours before the start of the experiment (i.e. $t=0$ hours, cf. Table 1), standard food was replaced by a $20 \%$ glucose drinking solution and water, and the animals were placed on a grid to minimize coprophagia.

ALI was induced the day after PCS by dividing the hepatic artery between two tightened ligatures after relaparotomy. Sham operations consisted of the same procedure, omitting hepatic artery ligation. Both procedures were completed within 8 minutes. At the end of the operation, $1 \mathrm{~mL}$ of $5 \%$ glucose solution was given intraperitoneally.

Hourly, in the period between $\mathrm{ALI} / \mathrm{sham}$ and sampling, the severity of $\mathrm{HE}$ was determined using a 6 stage coma scale $(0$, normal behavior; 5 , coma) $(5,25,26)$, rectal temperature was measured, and $1 \mathrm{~mL}$ of temperature adjusted $5 \%$ glucose solution was given intraperitoneally to prevent hypoglycemia $(4,25-27)$. Rectal temperature was maintained between $34^{\circ} \mathrm{C}$ and $36^{\circ} \mathrm{C}$ in ALI rats, using a heating lamp if necessary.

Blood sampling and flow measurements using the indicator dilution principle as described by Welbourne (28-30) were performed at the time intervals indicated in the figures and Table 1. Rats were anesthetized $30 \mathrm{~min}$ before sampling. Rectal temperature was monitored and maintained at pre-anesthesia levels, using a heating lamp, heating pads, and moist saline gauzes. A tertiary branch of the mesenteric vein, the portal vein, the inferior caval vein and the abdominal aorta were cannulated (both just above the bifurcation) with a 
25-gauge needle fitted to silastic tubing (Silastic Medical Grade Tubing $0.051 \mathrm{~cm} \mathrm{ID,} 0.094$ $\mathrm{cm}$ OD, Dow Coming Corporation, Medical Products, Midland, Michigan, U.S.A.) and cemented in place with a cyanoacrylate adhesive. For flow determinations, para-aminohippuric acid (PAH) (50 mM, isomolaric, pH 7.40; Sigma A 1422, St. Louis, MI, U.S.A.) was infused in the mesenteric and aorta catheters, starting with a priming dose of $0.1 \mathrm{~mL}$, followed by continuous infusion $\left(1.5 \mathrm{~mL} \cdot \mathrm{h}^{-1}\right.$ on each line; Minipuls 2 pump, Gilson France $\mathrm{SA}$, Villiers le Bel, France) including a 20 minute equilibration period. At the end of the equilibration period the right common carotid artery was catheterized (Intramedic Polyethylene Tubing PE- $50,0.058 \mathrm{~cm}$ ID, $0.096 \mathrm{~cm}$ OD, Clay Adams, Parsippany, NJ, U.S.A.). Blood was slowly aspirated $\left(1.3 \mathrm{~mL}\right.$ per vessel at a rate of $\left.1.0 \mathrm{~mL} \cdot \mathrm{min}^{-1}\right)$ from, subsequently, the portal vein, the inferior caval vein, and the common carotid artery. After each blood collection $0.9 \% \mathrm{NaCl}$ was infused via the catheters for volume substitution. Blood was collected in heparinized tubes (Microvette CB 1000, Sarstedt, D-5223 Nümbrecht, F.R.G.) and kept on ice until further processing within 5 minutes.

The above described catheter positions combined with the infusion of PAH permit simultaneous determination of blood flow and arteriovenous concentration difference (=flux) across the portal drained viscera (PDV) $(28,29,31,32)$ and hindquarter (HQ) $(30,33,34)$. Blood flow is calculated as described under "Calculations" from the PAH concentration difference in the same pair of blood samples used to estimate the concentration difference across hindquarter and portal drained viscera. This technique of metabolite exchange measurement across the PDV and HQ ( $\pm 75 \%$ muscle $w / w$ (33-35), representing $95 \%$ of metabolically active tissue $(34,35)$ ) has been extensively used to study intestinal $(11,28,29,31,32,36)$ and skeletal muscle metabolism in the rat $(11,30,33-35)$.

Immediately after sampling, the right gastrocnemius muscle was rapidly excised, freeze clamped with Wollenberger tongs (total procedure in less than 30 seconds) (37), put into liquid nitrogen, and stored at $-70^{\circ} \mathrm{C}$. At the end of the experiment rats were killed by an overdose of the anaesthetic.

During all procedures, accomplished promptly after collection of blood, the samples were kept on ice. Centrifugation was performed at $4^{\circ} \mathrm{C}, 5 \mathrm{~min}$ at $8,800 \mathrm{~g}$. Hematocrit was obtained with a microfuge. For glucose and lactate determinations $200 \mu \mathrm{L}$ of whole blood were centrifuged in NaF containing tubes (Microvette FH, CB 300, Sarstedt). Ammonia, urea and amino acids were determined in plasma. For amino acid analysis $100 \mu \mathrm{L}$ of plasma were deproteinized with $4 \mathrm{mg}$ sulfosalicylic acid (SSA). For PAH determinations $50 \mu \mathrm{L}$ of whole blood were added to $200 \mu \mathrm{L}$ of $10 \%$ trichloracetic acid, thoroughly mixed, allowed to stand for deproteinization $\left(4^{\circ} \mathrm{C}, 30 \mathrm{~min}\right)$, and centrifuged after which the supernatant was collected. After these preparations all samples except PAH were frozen in liquid nitrogen. Hereafter, all samples were stored at $-70^{\circ} \mathrm{C}$ until further analysis.

Biochemical analyses Ammonia (38), glucose, lactate, and urea were determined spectrophotometrically by standard enzymatic methods on a centrifugal analyzer system (Cobas Bio, Roche Diagnostica, Hoffmann-La Roche, Basel, Switzerland) using commercial kits. Urea values were corrected for ammonia. The coefficient of variance (CV) for the plasma ammonia determinations was $4 \%$. Plasma amino acids were determined with a fully automated High Performance Liquid Chromatography system after pre-column derivatization with ortho-phthaldialdehyde as described previously (39). The CV for the plasma amino acid determinations was approximately $3 \%$ (e.g. glutamine $2 \%$, alanine $3 \%$ ) 
Table 2. Arterial concentrations of Urea, Glucose (GlC), and Lactate (Lact) and the fluxes of Glc and Lact.

Arterial plasma concentration ( $\mathrm{mmol} \cdot \mathrm{L}^{-1}$ )

\begin{tabular}{lrrrrrrrr} 
& \multicolumn{1}{c}{ NORM } & \multicolumn{1}{c}{ PCSO } & \multicolumn{1}{c}{ PCS2 } & \multicolumn{1}{c}{ PCS4 } & \multicolumn{1}{c}{ PCS6 } & \multicolumn{1}{c}{ ALl2 } & \multicolumn{1}{c}{ ALI4 } & \multicolumn{1}{c}{ ALl6 } \\
\hline Urea & $6.2 \pm 1.0$ & $6.8 \pm 0.5$ & $7.6 \pm 0.5$ & $7.4 \pm 0.4$ & $6.6 \pm 0.4$ & $5.4 \pm 0.2$ & $4.8 \pm 0.4^{\circ}$ & $5.2 \pm 0.5^{16}$ \\
Glc & $17.5 \pm 1.9$ & $12.4 \pm 1.6$ & $12.4 \pm 0.9$ & $10.6 \pm 0.5$ & $12.2 \pm 0.7$ & $7.3 \pm 0.4$ & $5.6 \pm 0.8$ & $4.6^{\circ} \pm 0.3^{30}$ \\
Lact & $7.5 \pm 0.4$ & $10.8 \pm 0.8^{\circ}$ & $10.0 \pm 0.8$ & $7.8 \pm 0.9$ & $6.7 \pm 0.8^{\circ}$ & $12.1 \pm 0.9$ & $9.6 \pm 0.6$ & $9.7 \pm 0.6^{\circ}$ \\
\hline
\end{tabular}

PDV fluxes

\begin{tabular}{lcccccccc} 
& NORM & PCS0 & \multicolumn{1}{c}{ PCS2 } & PCS4 & PCS6 & \multicolumn{1}{c}{ ALl2 } & AL/4 & ALl6 \\
\hline Glc & $-0.0 \pm 1.8$ & $-1.2 \pm 0.8$ & $-1.9 \pm 0.8^{+}$ & $-0.3 \pm 0.4$ & $-1.7 \pm 1.2$ & $1.8 \pm 1.4$ & $-0.1 \pm 0.1$ & $1.4 \pm 0.4^{\text {H }}$ \\
Lact & $-0.9 \pm 0.5$ & $0.7 \pm 0.8$ & $-0.8 \pm 1.3$ & $1.1 \pm 0.4$ & $0.8 \pm 0.8$ & $0.6 \pm 0.4$ & $-0.1 \pm 0.3$ & $-0.3 \pm 0.5$ \\
\hline
\end{tabular}

HQ fluxes

\begin{tabular}{ccccccccc} 
& NORM & PCSO & PCS2 & PCS4 & PCS6 & AL/2 & AL/4 & ALl6 \\
\hline Glc & $-1.4 \pm 0.9$ & $-0.7 \pm 0.4$ & $-2.3 \pm 1.0$ & $-0.8 \pm 0.6$ & $-2.8 \pm 1.2^{+}$ & $1.9 \pm 1.4$ & $-0.3 \pm 0.5$ & $0.1 \pm 0.2^{*}$ \\
Lact & $-0.1 \pm 0.6$ & $1.3 \pm 0.4^{*}$ & $2.4 \pm 0.4^{+}$ & $1.5 \pm 0.5$ & $3.0 \pm 0.7^{+}$ & $2.1 \pm 0.7^{+}$ & $0.8 \pm 0.4$ & $-0.2 \pm 0.1^{*}$
\end{tabular}

Fluxes are in $\mu \mathrm{mol}$ min $^{-1} \cdot 100 \mathrm{~g} \mathrm{bw^{ \prime }}$. Significances are indicated as follows: ANOVA for differences between normal control rats and PCSO, and between ALI and PCS; $t=0$ to $t=6 h$ : symbols: " $P<0.05,{ }^{\circ} \mathrm{P}<0.01,{ }^{\mathrm{C}} \mathrm{P}<0.001$. For time effects in PCS and $\mathrm{ALI}$ groups: 'P<0.05, ${ }^{2} P<0.01,{ }^{3} P<0.001$. Scheffé comparison per group: versus to, "versus $t_{2}$, versus 14 . Difference from zero for the fluxes was tested by the Wilcoxon test: ${ }^{+}<<0.05$.

(39). For PAH-determination the method according to Brun (40) was adapted for small volumes. $100 \mu \mathrm{L}$ Of the supernatant (obtained as described above), $300 \mu \mathrm{L}$ of milliQ water (demineralized, thrice distilled water), and $100 \mu \mathrm{L} \mathrm{HCl} 1.2 \mathrm{~N}$ were pipetted into screwtopped tubes, which were closed tightly, well mixed and cooked in a boiling water bath for deacetylation $\left(100^{\circ} \mathrm{C}, 1\right.$ hour). For photometric PAH-measurement (Cobas Bio, $465 \mathrm{~nm}$ ) $100 \mu \mathrm{L}$ of Ehrlich's reagent ( $1 \mathrm{~g} \mathrm{p}$-dimethyl-aminobenzaldehyde (Sigma D 2004), $60 \mathrm{~mL}$ alcohol 93 percent, $4 \mathrm{~mL}$ HCL $2 \mathrm{~N}$ and distilled water added to a final volume of $100 \mathrm{~mL}$ ) were added to $50 \mu \mathrm{L}$ sample and $30 \mu \mathrm{L}$ distilled water. PAH dissolved in milliQ water was used for calibration. The CV for the PAH determinations was $1 \%$.

Tissue processing The frozen muscle specimens were mechanically fragmented with a mortar and pestle, which were continuously cooled to the temperature of liquid nitrogen. Any visible connective tissue was removed with a precooled pair of tweezers. Approximately 0.5 gram of tissue powder was added to a pre-weighed vial containing $1.5 \mathrm{~mL}$ of $5 \%$ SSA at $-10^{\circ} \mathrm{C}$. Norvaline (Art. nr. $37162, \mathrm{BDH}$ Chemicals Ltd., Poole, England) was added to a final concentration of $0.5 \mathrm{mM}$ as internal standard for the amino acids determinations. Tissue weights were determined by re-weighing the vial. The tissue fragments were immediately homogenized with an Ultra Turrax (Janke and Kunkel, IKA-Labortechnik, Staufen i. Br., F.R.G.) at $-10^{\circ} \mathrm{C}$ for 30 seconds ( 5 seconds on/off). To remove adherent tissue, the Ultra Turrax was flushed with $0.5 \mathrm{~mL}$ of $5 \% \mathrm{SSA}$ at $-10^{\circ} \mathrm{C}$, which was added to the homogenate. The homogenate was allowed to stand for 5 minutes at $-10^{\circ} \mathrm{C}$ for deproteinization and then frozen in liquid nitrogen and stored at $-70^{\circ} \mathrm{C}$.

For ammonia and amino acid determinations, the homogenates were thawed and centrifuged $(8,800 \mathrm{~g})$ at $4^{\circ} \mathrm{C}$ for 5 minutes. The supernatant was again centrifuged as before and 


\section{Chapter 5}

Table 3. Arterial plasma concentrations.

\begin{tabular}{|c|c|c|c|c|c|c|c|c|}
\hline & NORM & PCSO & PCS2 & PCS4 & PCS6 & $\mathrm{ALI} 2$ & $\mathrm{AL} 14$ & ALI6 \\
\hline Amm & $147 \pm 14$ & $13 \pm 27^{c}$ & $334 \pm 27$ & $183 \pm 31^{\prime \prime}$ & $193 \pm 14^{3 \cdot 1}$ & $815 \pm 78$ & $1046 \pm 57$ & $1555 \pm 147$ \\
\hline GLN & $3+23$ & $5 \pm 53^{c}$ & $3 \pm 43$ & $646 \pm 35$ & 694 & $1852 \pm 112^{\circ}$ & $2319 \pm 91^{\circ}$ & $3177 \pm 294$ \\
\hline GLU & $15 \pm 15$ & $24 \pm 17$ & $92 \pm 11$ & $95 \pm 10$ & $89 \pm 11$ & $193 \pm 33$ & $225 \pm 14$ & $438 \pm 49$ \\
\hline SN & $50 \pm 3$ & $95 \pm 3^{c}$ & $5 \pm 5$ & $7 \pm 4$ & $96 \pm 10$ & $211 \pm 17^{\circ}$ & $258 \pm 14^{\circ}$ & $281 \pm 11$ \\
\hline ER & $161 \pm 7$ & $00 \pm 8^{\circ}$ & $83 \pm 10$ & $190 \pm 10$ & $195 \pm 18$ & $522 \pm 41^{\circ}$ & $848 \pm 60^{\circ}$ & $1352 \pm 60^{\circ 3}$ \\
\hline GLY & $254 \pm 11$ & $270 \pm 13$ & $268 \pm 12$ & $236 \pm 15$ & $252 \pm 23$ & $793 \pm 64^{\circ}$ & $1385 \pm 85^{\prime \prime}$ & $1811 \pm 148$ \\
\hline THR & $124 \pm 10$ & $188 \pm 9^{b}$ & $165 \pm 4$ & $158 \pm 6$ & $162 \pm 17$ & $425 \pm 31^{\circ}$ & $691 \pm 37^{\prime \prime}$ & $1085 \pm 55$ \\
\hline HIS & $94 \pm 14$ & $8 \pm 9$ & $06 \pm 14$ & $05 \pm 13$ & $102 \pm 11$ & $186 \pm 11^{\circ}$ & $258 \pm 7$ & $223 \pm 14$ \\
\hline CIT & $46 \pm 2$ & $58 \pm 2^{\circ}$ & $4 \pm 3$ & $38 \pm 3^{\prime \prime}$ & $46 \pm 3^{3}$ & $112 \pm 10^{\circ}$ & $118 \pm 7^{\circ}$ & $111 \pm 11$ \\
\hline ALA & $8 \pm 27$ & $625 \pm 26^{c}$ & $550 \pm 30$ & $2 \pm 37$ & $585 \pm 58$ & $1763 \pm 172^{\circ}$ & $2648 \pm 150^{\circ}$ & $3107 \pm 196$ \\
\hline TAU & $6 \pm 7$ & $61 \pm 6^{c}$ & \pm 24 & \pm 26 & $215 \pm 25$ & $466 \pm 52$ & $684 \pm 89$ & $993 \pm 207$ \\
\hline $\mathrm{AG}$ & $126 \pm 8$ & $15 \pm 20$ & $127 \pm 19$ & $123 \pm 13$ & $132 \pm 12$ & $29 \pm 10$ & $3 \pm 1$ & $8 \pm \quad 4$ \\
\hline . & $11 \pm 2$ & $1 \pm 2$ & $5 \pm 5$ & $4 \pm 3$ & $13 \pm 4$ & $15 \pm 2$ & $20 \pm 3$ & $30 \pm 2$ \\
\hline YR & $87 \pm 4$ & $3 \pm 4$ & $1 \pm 7$ & \pm 4 & $85 \pm 9$ & $207 \pm 15^{\circ}$ & $339 \pm 20^{\prime}$ & $534 \pm \quad 8^{630}$ \\
\hline VAL & $104 \pm 6$ & $132 \pm 5^{2}$ & $4 \pm 9$ & \pm 6 & $145 \pm 15$ & $4 \pm 15$ & $292 \pm 24^{\prime \prime}$ & $457 \pm 25$ \\
\hline AET & $35 \pm 2$ & $56 \pm 2^{c}$ & $48 \pm 2$ & $13 \pm 2$ & $51 \pm 6$ & $143 \pm 12^{\circ}$ & $257 \pm 18^{\prime}$ & $443 \pm 14$ \\
\hline ILE & $52 \pm 3$ & $67 \pm 3^{a}$ & $69 \pm 5$ & $74 \pm 4$ & $72 \pm 8$ & $97 \pm 8$ & $155 \pm 11$ & $222 \pm 14$ \\
\hline PHE & $52 \pm 2$ & $101 \pm 4^{c}$ & $98 \pm 5$ & $93 \pm 3$ & $104 \pm 10$ & $210 \pm 16^{\circ}$ & $379 \pm 27$ & $651 \pm 23^{63 *}$ \\
\hline TRP & $35 \pm 2$ & $57 \pm 2^{c}$ & $49 \pm 3$ & $51 \pm 2$ & $55 \pm 4$ & $62 \pm 4$ & $72 \pm 3$ & $82 \pm 6$ \\
\hline LEU & $75 \pm 5$ & $96 \pm 4^{4}$ & $108 \pm 10$ & $112 \pm 6$ & $106 \pm 11$ & $151 \pm 12$ & $238 \pm 17^{\prime}$ & $370 \pm 24$ \\
\hline LYS & $303 \pm 15$ & $332 \pm 13$ & $317 \pm 16$ & $302 \pm 14$ & $297 \pm 30$ & $1188 \pm 91$ & $2492 \pm 202$ & $4553 \pm 596$ \\
\hline BCAA & $232 \pm 13$ & $295 \pm 11^{8}$ & $310 \pm 24$ & $335 \pm 15$ & $323 \pm 34$ & $432 \pm 35$ & $684 \pm 52^{\prime \prime}$ & $1049 \pm 61$ \\
\hline
\end{tabular}

Units of measure are $\mu \mathrm{mol} \cdot \mathrm{L}^{-1}$. NORM: normal control rats. Significances are indicated as follows: ANOVA for differences between normal control rats and PCSO, and between $A L I$ and $P C S ; 1=0$ to $t=6$ h: symbols: ${ }^{\mathrm{P}} \mathrm{P}<0.05,{ }^{\mathrm{D}} \mathrm{P}<0.01,{ }^{\mathrm{C}} \mathrm{P}<0.001$. For time effects in PCS and ALI groups: $P<0.05,{ }^{2} \mathrm{P}<0.01,{ }^{3} \mathrm{P}<0.001$. Scheffé comparison per group: versus 10 , 'versus $\mathrm{Z}$, versus 14. $\alpha$-Amino adipic acid ( $\alpha$ AAA) (slightly higher in ALI rats) is not shown.

divided in portions of $200 \mu \mathrm{L}$. Hereafter, they were neutralized with $30 \mu \mathrm{L}$ of $2 \mathrm{M} \mathrm{KHCO}_{3}$ to a pH of approximately 5.0 and immediately determined (ammonia) or stored (amino acids) at $-70^{\circ} \mathrm{C}$ until analysis.

Calculations $\alpha$-Amino nitrogen was calculated by adding the concentrations of the individual amino acids measured (39). BCAA were calculated as the sum of the branched chain amino acids: valine, leucine and isoleucine. Blood flow over HQ and PDV was calculated using the formula introduced by Katz and Bergmann (41), based on the Fick principle for indicator dilution methods:

$$
F_{M, P}=\frac{100 \times 1}{\left([P A H]_{P, V}-[P A H]_{A}\right) \times \text { body weight }(b W)}
$$

where $\mathrm{F}_{\mathrm{H}, \mathrm{P}}$ is blood flow $\left(\mathrm{mL} \mathrm{min}^{-1} \cdot 100 \mathrm{~g} \mathrm{bw}^{-1}\right)$ over HQ $(\mathrm{H})$ or PDV $(\mathrm{P})$, I is the infused $\mathrm{PAH}\left(\mu \mathrm{mol} \cdot \mathrm{min}^{-1}\right)$, and $[\mathrm{PAH}]_{\mathrm{A} . \mathrm{P} . \mathrm{V}}$ are the concentrations of $\mathrm{PAH}\left(\mu \mathrm{mol} \cdot \mathrm{L}^{-1}\right)$ in portal $(\mathrm{P})$, venous $(\mathrm{V})$, and arterial (A) blood, respectively. Plasma flows were calculated as follows: Flow $_{\text {plasma }}=(1-$ hematocrit $) \cdot$ Flow $_{\text {biood }}$. 
Table 4. Portal drained viscera fluxes.

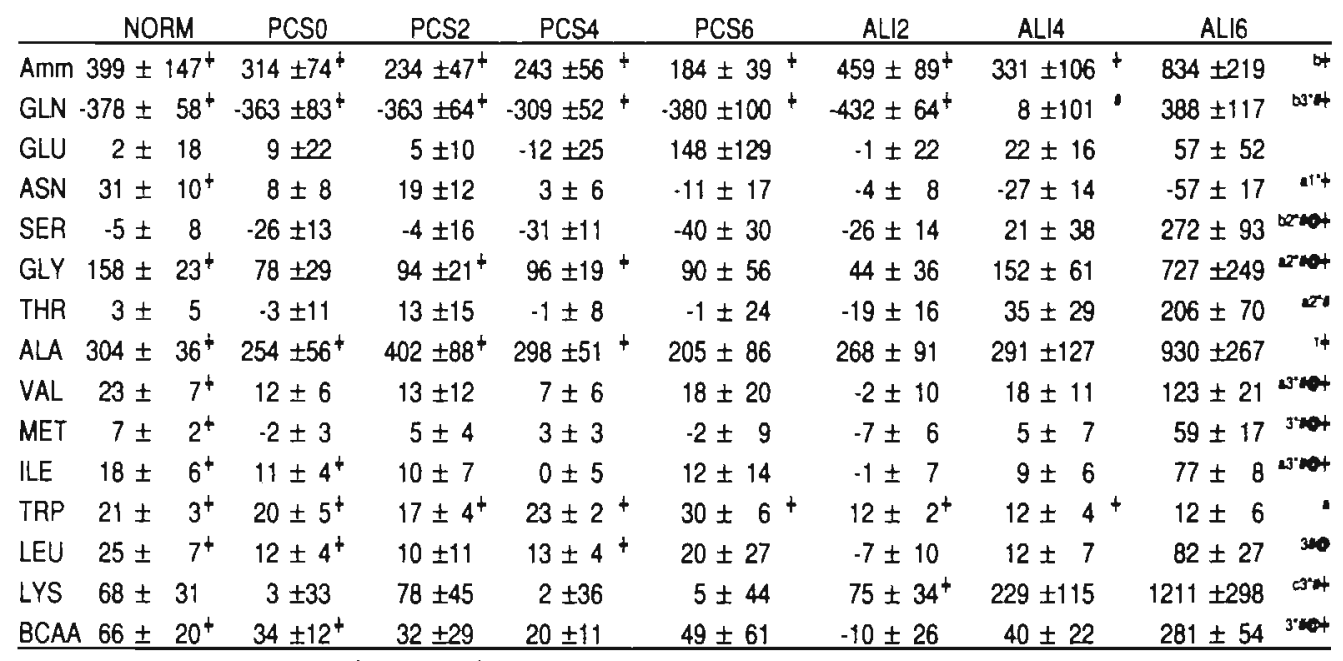

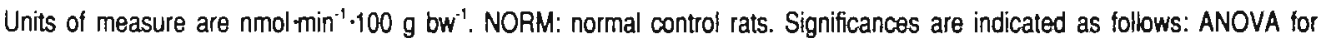
differences between normal control rats and PCSO, and between ALI and PCS; $t=0$ to $t=6 h$ : symbols: $P<0.05,{ }^{b} P<0.01$, ${ }^{5} P<0.001$. For time effects in $P C S$ and $A L I$ groups: ${ }^{1} P<0.05,{ }^{2} P<0.01,{ }^{3} P<0.001$. Scheffé comparison per group: versus to, "versus $L_{2}$, versus 14 . Difference from zero was tested by the Wilcoxon test: ${ }^{+} P<0.05$. Not shown: $\alpha A A A, H I S, A R G, \alpha A B A$, TYR, PHE (not different from zero in most groups, no changes), CIT, TAU (constant release of approx. 60 respectively 120 $\left.\mathrm{nmol} \cdot \min ^{-1} \cdot 100 \mathrm{~g} \mathrm{bW}^{-1}\right)$.

Fluxes were calculated as the mean flow per group times the individual V-A and P-A differences and expressed per $100 \mathrm{~g}$ of body weight (bw) per min (mean flow was used because flow data were not available for all rats). A positive figure means net efflux, negative values reflect net uptake.

Statistical analysis Results are presented as means \pm 1 SEM. Levels of significance were set at $\mathrm{P}<0.05$ unless stated otherwise. For statistical analysis (42) analysis of variance (ANOVA) was used for comparison of differences between normal control rats and PCS0, and ALI and PCS groups, respectively, and for analysis of time effects. Individual group comparison was done by the Scheffé multiple comparison test after testing for time effects. Significance from zero was tested by the Wilcoxon test.

\section{RESULTS}

General Plasma PDV flow (Figure 1) in normal rats was comparable with literature data $(23,28,29,31)$, as was HQ plasma flow $(11,30,33)$. PDV plasma flow did not differ significantly between the groups. Only HQ flow in ALI rats at $t=6$ hours was significantly reduced compared with the PCS $t=6$ hours group, but not to normal rats. ALI rats were on the average in HE phase 1.5 (lethargy, slightly impaired postural control) at $t=2$ hours and in HE phase 2.5 (severe ataxia) at $\mathrm{t}=6$ hours $(\mathrm{P}<0.001)$ (data not shown). 
Table 5. Hindquarter fluxes.

\begin{tabular}{|c|c|c|c|c|c|c|c|c|c|}
\hline & NORM & PCSO & $\mathrm{PCS} 2$ & PCS4 & PCS6 & & $\mathrm{ALI} / 2$ & ALI4 & $A \leq 16$ \\
\hline Amm & $73 \pm 24^{4}$ & $34 \pm 16$ & $-46 \pm 49$ & $25 \pm 14$ & $11 \pm 36$ & & $-23 \pm 75$ & $-22 \pm 59$ & $-46 \pm 38$ \\
\hline GLN & $153 \pm 33^{+}$ & $348 \pm 20^{\circ}$ & $459 \pm 45^{t}$ & $324 \pm 61+$ & $691 \pm 94^{26}$ & & $66 \pm 123$ & $268 \pm 164$ & $40 \pm 162$ \\
\hline GLU & $-52 \pm 17^{+}$ & $-15 \pm 16$ & $5 \pm 27$ & $-23 \pm 29$ & $166 \pm 188$ & & $-74 \pm 31$ & $-36 \pm 46$ & $-21 \pm 29$ \\
\hline SER & $19 \pm 11$ & $28 \pm 6^{+}$ & $40 \pm 17$ & $12 \pm 17$ & $45 \pm 29$ & & $-90 \pm 29^{+}$ & $-57 \pm 44$ & $3 \pm 56$ \\
\hline GLY & $77 \pm 15^{+}$ & $74 \pm 10^{+}$ & $152 \pm 19^{t}$ & $112 \pm 27^{+}$ & $208 \pm 47$ & it & $-14 \pm 42$ & $246 \pm 63+$ & $183 \pm 138$ \\
\hline THR & $19 \pm 7^{\dagger}$ & $40 \pm 5^{+}$ & $72 \pm 15^{\dagger}$ & $40 \pm 11+$ & $85 \pm 22$ & + & $-6 \pm 29$ & $40 \pm 36$ & $30 \pm 31$ \\
\hline ALA & $27 \pm 40$ & $24 \pm 27$ & $98 \pm 56$ & $78 \pm 55$ & $165 \pm 123$ & & $-273 \pm 114$ & $-195 \pm 132$ & $61 \pm 79$ \\
\hline TAU & $37 \pm 13^{+}$ & $32 \pm 8$ & $104 \pm 31^{+}$ & $53 \pm 9^{+}$ & $-22 \pm 66$ & & $102 \pm 31^{t}$ & $249 \pm 53^{\prime}$ & $80 \pm 36$ \\
\hline ARG & $11 \pm 11$ & $21 \pm 7$ & $58 \pm 24$ & $43 \pm 28$ & $-6 \pm 67$ & & $-52 \pm 16^{\prime}$ & $20 \pm 13$ & $3 \pm 6$ \\
\hline VAL & $-16 \pm 4^{+}$ & $-4 \pm 5$ & $-13 \pm 13$ & $-1 \pm 6$ & $3 \pm 17$ & & $-16 \pm 15$ & $1 \pm 17$ & $16 \pm 9$ \\
\hline MET & $2 \pm 2$ & $10 \pm 2^{+}$ & $18 \pm 5^{+}$ & $12 \pm 2^{+}$ & $23 \pm 6$ & + & $-5 \pm 9$ & $2 \pm 12$ & $22 \pm 6$ \\
\hline ILE & $-18 \pm 4^{+}$ & $-5 \pm 3$ & $-8 \pm 6$ & $-10 \pm 4$ & $-5 \pm 13$ & & $-17 \pm 8$ & $-7 \pm 10$ & $8 \pm 5$ \\
\hline LEU & $-23 \pm 6^{+}$ & $-6 \pm 3$ & $-22 \pm 13$ & $-1 \pm 4$ & $4 \pm 23$ & & $-21 \pm 14$ & $-2 \pm 14$ & $4 \pm 11$ \\
\hline LYS & $34 \pm 26$ & $-79 \pm 114$ & $205 \pm 60^{+}$ & $108 \pm 28+$ & $167 \pm 77$ & & $85 \pm 100$ & $494 \pm 135+$ & $737 \pm 226^{a 2+}$ \\
\hline BCAA & $-57 \pm 14^{+}$ & $-15 \pm g^{2}$ & $-43 \pm 31$ & $-12 \pm 10$ & $2 \pm 52$ & & $-54 \pm 35$ & $-8 \pm 38$ & $28 \pm 23$ \\
\hline
\end{tabular}

Units of measure are nmol $\cdot \mathrm{min}^{-1} \cdot 100 \mathrm{~g} \mathrm{bw}^{-1}$. NORM: normal control rats. Significances are indicated as follows: ANOVA for differences between normal control rats and PCSO, and between $A L I$ and $P C S ; t=0$ to $t=6 h$ : symbols: ${ }^{\circ} P<0.05,{ }^{D} P<0.01$, ${ }^{\mathrm{C}} \mathrm{P}<0.001$. For time effects in $\mathrm{PCS}$ and $\mathrm{ALI}$ groups: ${ }^{\mathrm{P}} \mathrm{P}<0.05,{ }^{2} \mathrm{P}<0.01,{ }^{3} \mathrm{P}<0.001$. Scheffe comparison per group: versus to, 'versus 12 , versus 14 . Difference from zero was tested by the Wilcoxon test: ${ }^{+} P<0.05$. Not shown: $\alpha A A A, C I T, H I S, \alpha A B A$, TYA, PHE, TAP (not different from zero in most groups, no changes), ASN (constant release of $\pm 30 \mathrm{nmol}^{-1 n^{-1} \cdot 100 \mathrm{~g} \mathrm{bw}} \mathrm{w}^{-1}$ ).

Arterial values Arterial urea values (Table 2) decreased significantly in ALI rats compared with normal control rats and PCS groups. Arterial glucose concentration was significantly higher in normal control rats, compared with PCS rats at $t=2,4$, and 6 hours, but not at $t=0$ hours. It decreased significantly in ALI rats $(P<0.001)$, but was within the normal range $\left(3-10 \mathrm{mmol} \cdot \mathrm{L}^{-1}\right)$. Arterial lactate was significantly higher in PCS0 than in normal control rats. In PCS2, PCS4 and PCS6 rats, lactate decreased in time. In ALI rats, lactate was significantly higher than in PCS rats. Arterial ammonia (Figure 2) was significantly increased in PCS compared with normal control rats at $t=0$ hours $(\mathrm{P}<0.001)$. In PCS rats a time related decrease in ammonia was observed $(\mathrm{P}<0.001)$. In ALI rats, ammonia increased progressively 2 to 6 -fold in time compared with PCS rats $(\mathrm{P}<0.001)$. Glutamine and most other plasma amino acids (Table 3 ) were significantly elevated in PCS compared with normal control rats. In ALI rats a more or less pronounced linear increase in time was seen for glutamine and most other amino acids $(\mathrm{P}<0.001)$, arginine decreased $(\mathrm{P}<0.001)$.

Portal drained viscera fluxes PDV glucose flux (Table 2) was not significantly different in PCS rats compared with normal control rats and was not significantly different from zero at any time point except for PCS at $t=2$ hours and ALI at $t=6$ hours. PDV lactate flux did not differ from zero at any time point.

Net ammonia efflux (Figure 2) in PCS rats was not significantly different from normal control rats. Significantly more ammonia was released in the ALI groups than in PCS groups between $t=0$ hours and $t=6$ hours $(P<0.01$ ) (Figure 2). The net PDV fluxes (Table 4) of all amino acids were not different in PCS compared with normal control rats. The PDV efflux of most essential amino acids (except taurine, phenylalanine and tyrosine) was significantly 
Table 6. Gastrocnemius muscle tissue concentrations.

\begin{tabular}{|c|c|c|c|c|c|c|c|c|}
\hline & NORM & PCSO & $\operatorname{cs} 2$ & $\mathrm{CS} 4$ & PCS6 & ALL2 & $\mathrm{AL} / 4$ & ALL16 \\
\hline mm & $787 \pm 93$ & $1221 \pm 231^{\circ}$ & $1126 \pm 153$ & $805 \pm 192$ & $615 \pm 58^{3}$ & $2246 \pm 549$ & $1336 \pm 198$ & $1433 \pm 120$ \\
\hline GLN & $3280 \pm 207$ & $4475 \pm 335^{\circ}$ & $3874 \pm 338$ & $14+258$ & $72 \pm 297$ & $5479 \pm 340$ & $6089 \pm 523$ & $9491 \pm 263$ w20 \\
\hline ER & $668 \pm 57$ & $615 \pm 68$ & $50 \pm 85$ & $38 \pm 46$ & $764 \pm 130$ & $1064 \pm 192$ & $893 \pm 85$ & $1524 \pm 113$ \\
\hline GLY & $3954 \pm 417$ & $2961 \pm 207$ & $3466 \pm 429$ & $3052 \pm 235$ & $92 \pm 162$ & $43 \pm 211$ & $3522 \pm 347$ & $3916 \pm 318$ \\
\hline$A\llcorner A$ & $1032 \pm 194$ & $1602 \pm 148^{a}$ & $1175 \pm 104$ & $1941 \pm 215^{\prime}$ & $1646 \pm 204^{\prime}$ & $1936 \pm 280$ & $2537 \pm 383$ & $3397 \pm 184$ \\
\hline TYA & $182 \pm 16$ & $218 \pm 34$ & $1 \pm 10$ & $176 \pm 8$ & $182 \pm 13$ & $08 \pm 40$ & $427 \pm 41^{\circ}$ & $706 \pm 41$ \\
\hline VAL & $142 \pm 22$ & $186 \pm 19$ & $154 \pm 12$ & $201 \pm 13$ & $201 \pm 29$ & $217 \pm 34$ & $268 \pm 33$ & $414 \pm 39^{+5}$ \\
\hline MET & $55 \pm 10$ & $103 \pm 23^{3}$ & $71 \pm 7$ & $86 \pm 9$ & $92 \pm 14$ & $66 \pm 26$ & $263 \pm 30^{\circ}$ & $468 \pm 28$ \\
\hline ILE & $55 \pm 9$ & $82 \pm 9^{a}$ & $66 \pm 8$ & $97 \pm 9$ & $84 \pm 13$ & $15 \pm 17$ & $118 \pm 16$ & $181 \pm 20$ \\
\hline PHE & $81 \pm 11$ & $167 \pm 31^{\circ}$ & $119 \pm 14$ & $145 \pm 7$ & $150 \pm 14$ & $40 \pm 32$ & $384 \pm 43^{\circ}$ & $674 \pm 36$ c34e \\
\hline LEU & $78 \pm 10$ & $119 \pm 23$ & $106 \pm 16$ & $134 \pm 9$ & $2 \pm 16$ & $35 \pm 40$ & 259 & $376 \pm 79$ \\
\hline IYS & $560 \pm 81$ & $392 \pm 37$ & $420 \pm 53$ & $269 \pm 78$ & $335 \pm 22$ & $7 \pm 57$ & $665 \pm 66^{\circ}$ & $1068 \pm 95^{\text {c3. }}$ \\
\hline BCAA & $275 \pm 38$ & $387 \pm 47$ & $325 \pm 34$ & $431 \pm 22$ & $418 \pm 57$ & $497 \pm 87$ & $645 \pm 74$ & $971 \pm 120$ \\
\hline$\alpha$ AN & $36.7 \pm 1.6$ & $39.1 \pm 1.0$ & $37.4 \pm 1.7$ & $39.4 \pm 1.0$ & $39.2 \pm 0.9$ & $44.7 \pm 4.5$ & $44.3 \pm 2.0$ & $51.4 \pm 1.1$ \\
\hline
\end{tabular}

Units of measure are $\mu$ mol $\mathrm{kg}^{-1}$ wet weight ( $\alpha \mathrm{AN}=\alpha-\mathrm{AminoN}$ in thousands). NORM: normal control rats. Significances are indicated as follows: ANOVA for differences between normal control rats and PCSO, and between ALI and PCS; $t=0$ to $t=6 h$ : symbols: ${ }^{a} P<0.05,{ }^{\circ} P<0.01,{ }^{~} P<0.001$. For time effects in $P C S$ and $A L I$ groups: ${ }^{P}<<0.05,{ }^{2} P<0.01,{ }^{3} P<0.001$. Scheffe comparison per group: versus t0, 'versus t2, "versus t4. Not shown: $\alpha A A A, \alpha A B A$ (not determined), ARG, HIS, TAU (approx. 1250,275 , and $21000 \mu \mathrm{mol} \mathrm{kg}^{-1}$ wet weight respectively, no changes), ASN, THR, TRP (same pattern as PHE) and CIT (higher levels in ALI than PCS rats (approx. 190 versus $150 \mu$ mol $\mathrm{kg}^{1}$ wet weight, time dependent decrease in both groups).

increased in ALI rats in comparison with PCS rats $(\mathrm{P}<0.001)$. $\alpha$-Amino nitrogen efflux (Figure 3) was not different between normal control and PCS rats. It was increased in ALI compared with PCS rats $(\mathrm{P}<0.01)$, and showed a time dependent increase in ALI rats $(\mathrm{P}<0.001)$. However, the most striking finding (Figure 4) was that the PDV switched from net glutamine uptake to net release between 2 and 6 hours after induction of liver ischemia $(\mathrm{P}<0.001)$.

Hindquarter fluxes HQ glucose uptake (Table 2) in PCS rats was not significantly different from normal control rats. In ALI rats, net glucose flux was not significantly different from zero at any time point. Lactate release was not significantly different in PCS rats compared with normal control rats. In ALI rats lactate release was significantly different from zero only at $t=2$ hours.

Also, HQ ammonia fluxes (Figure 2) were not significantly different between normal control and PCS rats, nor did they differ between ALI and PCS rats or from zero. Net glutamine release (Figure 4) was significantly higher $(\mathrm{P}<0.05)$ in PCS0 rats than in normal control rats, and increased in time ( $\mathrm{P}<0.01, \mathrm{PCS} 6$ vs. PCS0 and PCS4). Net glutamine efflux was significantly decreased in ALI rats compared with PCS rats $(\mathrm{P}<0.01)$ and was during the whole experiment not significantly different from zero. The fluxes of the other amino acids showed the following pattern: HQ lysine efflux (Table 5) increased significantly in ALI rats, BCAA uptake was decreased in PCSO compared with normal control rats, glycine release increased in time in PCS rats, arginine flux decreased in time in ALI rats, taurine flux increased in time in ALI rats, and serine and alanine fluxes were decreased in ALI compared 
with PCS rats. The remaining amino acids and $\alpha$-amino nitrogen fluxes (Figure 3 ) did not change significantly in any of the groups.

Gastrocnemius muscle tissue concentrations Tissue ammonia concentrations (Figure 5) were significantly increased in portacaval shunted (PCSO) as compared with normal control rats $(\mathrm{P}<0.05)$. In $\mathrm{PCS}$ rats, a time related decrease in tissue ammonia was observed $(\mathrm{P}<0.05)$. After liver ischemia induction, an initial increase in tissue ammonia was observed at $\mathrm{t}=2$ hours, which then decreased to a stable, but still elevated $(\mathrm{P}<0.01)$ level at $\mathrm{t}=4$ and 6 hours compared with both PCS and normal control rats.

Tissue glutamine concentrations (Figure 5) were increased in PCS rats as compared with controls ( $\mathrm{P}<0.01$ ). A further progressive increase was observed after liver ischemia induction $(\mathrm{P}<0.001)$. Glutamate concentrations (Figure 5) were increased in ALI rats compared with $\mathrm{PCS}$ rats $(\mathrm{P}<0.01)$ and increased in time during ALI $(\mathrm{P}<0.01)$. The majority of the other amino acid concentrations (Table 6) did not change following PCS, except asparagine, alanine, methionine, isoleucine, phenylalanine and tryptophan. During liver ischemia most amino acid concentrations increased progressively, except those of histidine, taurine and arginine.

\section{DISCUSSION}

It has been suggested repeatedly that during diminished or absent urea synthesis, ammonia detoxification by glutamine synthesis is enhanced in several glutamine synthetase containing organs, of which muscle, by virtue of its mass, has been suggested to be quantitatively most important $(3,6,10,43,44)$. Glutamine synthesis in glutamine synthetase containing organs can only contribute to net ammonia detoxification during diminished or absent urea synthesis capacity, if the produced glutamine is subsequently released from the organ, deaminated in the kidney and the ammonia excreted in the urine. Another possibility would be an increase of the tissue glutamine pools, but this would act merely as a temporary ammonia buffer.

Glutamine produced by muscle ammonia detoxification can be deaminated by the action of the enzyme glutaminase, quantitatively most important in enterocytes (45), and for this reason we investigated the metabolic interaction between a mainly glutamine consuming and a glutamine producing organ during liver insufficiency-induced hyperammonemia. The interaction between ammonia and glutamine metabolism in rat muscle and intestine during PCS and absent liver function was investigated by measuring their fluxes across the $H Q$, representing skeletal muscle $(35,46)$, and across the PDV, mainly small and large intestine (11), concomitant with muscle concentrations. 
The increased arterial glucose concentration in normal control rats and PCS rats probably is partly a result of the previous operation, which is comparable with the hyperglycemic response in postoperative patients. Furthermore, the intraperitoneal glucose injections and the $20 \%$ glucose drinking solution given to all animals, as well as the ether anesthesia (47), might contribute to the observed high glucose levels. The high lactate concentrations probably result from the ether anesthesia $(47,48)$ and the liver insufficiency $(27)$. Changes in glucose and lactate fluxes (Table 2) are small and only in some instances different from zero. Therefore, no definite conclusions are justified.

The arterial ammonia and amino acid concentrations are comparable with data during acute liver ischemia reported in literature $(5,25,49,50)$, including the observed decrease in plasma arginine concentration (50). The hyperaminoacidemia has been suggested to reflect lysis of necrotic liver tissue, protein breakdown in peripheral tissues and the absence of normal liver metabolic functions (51). The decreased arginine concentrations could be related to the increased brain arginase activity observed during acute $\mathrm{HE}$, possibly indicating increased participation of arginine in glutamate synthesis (52). Also, as the kidney is a main producer of arginine in the rat (13), renal arginine synthesis could be diminished during acute liver ischemia, but this has never been reported.

In our study, no significant net ammonia uptake or release across the HQ was found in any of the conditions studied (Figure 2). Although this does not exclude the possibility of equal amounts of ammonia entering and leaving skeletal muscle, it indicates that, in this model, muscle does not contribute to net ammonia detoxification of blood-derived ammonia. Glutamine release from the $\mathrm{HQ}$ in normal control rats was comparable with literature data (11) and was found elevated in PCS rats. During acute liver ischemia glutamine release decreased within 2 hours to values not significantly different from zero (Figure 4). This decrease was mainly caused by a decreased arteriovenous concentration difference, since blood flow was maintained until $t=4$ hours. Even at $t=6$ hours, the 17 -fold (!) decreased glutamine efflux could only partly be explained by the 3-fold decreased flow; the remainder resided in a decreased arteriovenous concentration difference, suggesting impaired glutamine exchange.

Therefore, in our experimental animal model of hyperammonemia, rising ammonia concentrations neither evoked increased HQ ammonia uptake nor enhanced HQ glutamine 
release (Figure 2 and 4). Because about $95 \%$ of metabolically active tissue in the hindquarter consists of skeletal muscle $(34,35)$, and during conditions favoring enhanced glutamine synthesis and release in skeletal muscle (e.g. after thermal injury and during glucocorticoid administration), skin and fat also release glutamine $(33,34)$, it seems reasonable to assume that the observed changes in HQ fluxes represent changes in muscle metabolism.

The findings concerning muscle metabolism contrast with several studies reported in the literature. In patients suffering from hepatic insufficiency, muscle extracts ammonia $(3,6-8)$. This ammonia uptake was found either correlated $(6,7)$ or unrelated $(8)$ to the arterial ammonia concentration. Although increased skeletal muscle glutamine efflux was found in patients with liver cirnhosis (6), it is unclear how this is related to net muscle ammonia uptake. During in vivo experiments under physiological conditions, ammonia infusion in dogs $(53,54)$, monkeys $(53)$, rats $(12,55)$ and pigs $(56)$ induced enhanced net skeletal muscle ammonia uptake. The enhanced net ammonia uptake observed in these studies coincided with unchanged (54) or enhanced glutamine release $(53,55,56)$. Ammonia uptake either exceeded (56) or balanced the enhanced glutamine release (53). The lacking stoichiometry underlines the importance of distinguishing between mere ammonia trapping and ammonia related glutamine synthesis with intracellular glutamine accumulation and possibly muscle glutamine release. Moreover, any attempt to verify muscle glutamine synthesis from blood-borne ammonia must take into account the commonly observed muscle glutamine release during catabolic states $(33,45,57)$. This glutamine release possibly is caused by changes in muscle protein turnover and/or release from

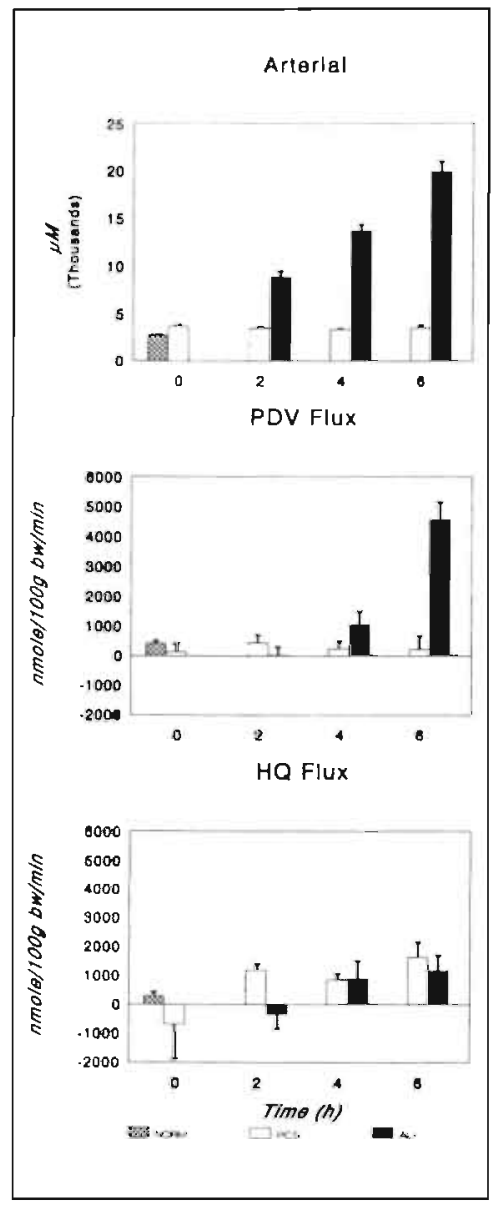

Figure 3. Total $\alpha$-Amino Nitrogen: arterial concentrations in $\mu$ mol $L^{-1}$ (top), PDV (middle) and $H Q$ (bottom) fluxes in nmol min ${ }^{-1} \cdot 100 \mathrm{~g} \mathrm{bw}^{-1}$ (ct. Table 1). Normal control rats: hatched bars, PCS: open bars, ALI: filled bars. Values are means \pm SEM. the free glutamine pool (57-59). Therefore, because we did not find any significant net ammonia uptake by the HQ, an important role of skeletal muscle in net ammonia detoxification during acute liver insufficiency-induced hyperammonemia seems doubtful.

During severe and progressive hyperammonemia in the ALI rats, HQ glutamine efflux did not increase (Figure 4). This could be explained by the fact that the muscle ammonia concentrations occurring during PCS are already much higher than the Km of glutamine synthetase for ammonia $(44,60)$. However, contrary to the expectations, glutamine release decreased. The simultaneously increased tissue glutamine concentrations (Figure 5) may result either from continuing glutamine synthesis or protein breakdown, or a combination of these processes $(58,59,61)$. This observation suggests that during ALI the release of 
glutamine from skeletal muscle is inhibited. A possible explanation could reside in factors influencing transmembranous glutamine transport by the glutamine carrier. The high ammonia levels, influencing membrane potential, cation pumps, or intracellular $\mathrm{pH}$ could play a role in this respect (62) (Rennie MJ, Personal Communication, 1990).

As shown in Figure 5, the tissue ammonia concentrations rise after PCS. The observed time-related decrease of tissue ammonia concentrations in the PCS 2, 4 and 6 groups could be the result of equilibration with lower arterial ammonia concentrations in these groups, possibly related to prolonged fasting and slightly decreased postoperative PDV ammonia release.

Tissue glutamate concentration remained constant during the entire experiment, except in ALI rats at $\mathrm{t}=6$ hours. This seems to indicate that during our experiment, muscle glutamate shortage did not occur.

The rising amino acids concentrations in skeletal muscle during liver ischemia, combined with the elevation of the aromatic amino acids, and the increased efflux of most amino acids, suggests that in this situation net protein degradation does occur, suggesting that the increased muscle glutamine concentration probably partly arises from protein breakdown.

HQ glutamine efflux is increased 2- to 4-fold in the PCS groups compared with normal control rats, while a corresponding net ammonia uptake is not observed. Therefore, the amide of glutamine must at least partly originate from either protein or nucleotide degradation, from transamination reactions, or a combination. The increased tissue phenylalanine concentrations found in PCS rats ( $t=2,4$, and 6 hours), coinciding with unaltered HQ phenylalanine fluxes, could reflect an increased rate of net protein degradation, because phenylalanine can neither be synthesized nor degraded in skeletal muscle $(63,64)$. Therefore, enhanced muscle catabolism $(45,64)$ seems to be the most likely cause of this enhanced glutamine efflux.

During PCS as well as ALI, lysine was released from the HQ in large quantities. Although unexpected, this can be accounted for by the percentage lysine in skeletal muscle protein (approximately 10\%) (61). Tissue lysine concentrations doubled during ALI. Tissue concentrations of phenylalanine, another amino acid not degraded or synthesized in skeletal muscle (63), increased eightfold during ALI, although it constitutes only approximately $3 \%$ of muscle protein (61). Considering their respective relative abundance in muscle protein, the more pronounced increase in tissue phenylalanine concentration compared with lysine 
seems to be in agreement with their respective releases and suggests altered membrane transport. Similar changes in lysine flux were observed in isolated perfused lambda-carrageenan wounded rat hindlimbs (63).

The PDV glutamine uptake in normal rats observed in our experiments is in good agreement with literature data $(28,29,31)$. In PCS and normal control rats, PDV glutamine uptake is comparable, while ammonia efflux decreases in PCS rats after operation. From the data of normal control and PCS rats (Figure 2 and 4), it is obvious that net PDV glutamine uptake and ammoniagenesis is not enhanced at these increased arterial glutamine concentrations. This is in agreement with literature data in which in vitro intestinal glutamine uptake increased linearly to reach a maximum at glutamine concentrations of $600 \mu \mathrm{mol} \cdot \mathrm{L}^{-1}$ (18). However, a threefold increased net PDV glutamine uptake during glutamine infusion in the rat was reported (23), coinciding with constant net ammonia efflux, suggesting glutamine was retained in the PDV. Both glutamine synthesis $(13,18,32)$ and glutamine deamidation take place in the intestine $(17,32)$, although glutamine synthetase activity is low $(28,32,44)$. Also, although intracellular accumulation or release from intracellular stores may occur, the free glutamine pool in mucosal epithelial cells is small (13,32). Thus, net PDV glutamine uptake must be viewed as a result of glutamine consumption, synthesis, retainment or a combination. Their relative contribution can not be derived from the arteriovenous differences or from flux measurements. Therefore, our data can not elucidate the discrepancy in these literature data.

The most striking finding is that the PDV switch from net glutamine uptake to release in spite of rising arterial glutamine levels between 2 and 6 hours after inducing ALI. Glutamine uptake ceases at 4 hours after ALI, and turns into net release. Although enhanced glutamine synthesis may theoretically cause this change, the low activity and $\mathrm{Km}$ for ammonia of glutamine synthetase in the intestine $(28,44,60)$ makes this unlikely. Decreased net glutamine consumption due to inhibition of intestinal glutaminase by the arterial ammonia levels exceeding $1 \mathrm{mmol}$ could be an explanation $(21,65)$.

Intestinal glutaminase is almost exclusively enterocytic in localization $(13,17)$. Because glutamine consumption is essential for the maintenance of normal enterocytic function (66-70), glutaminase inhibition could induce a severe perturbation of the metabolism of the enterocyte. Diminished gut glutamine utilization has been suggested to cause detrimental changes in mucosal architecture (36,71). Similarly, ammonia has been shown to shorten the life span of the enterocyte in vitro $(72,73)$. Therefore, the huge increase of the PDV amino 
acid efflux in ALI rats at $t=6$ hours (Figure 3 ) can be caused by lysis of the cells, which could also explain the net efflux of glutamine by the PDV (45). The coinciding ammonia release at $t=6$ hours in ALI rats could be a result of enhanced uptake from the lumen. Apart from these metabolic explanations, it cannot be excluded that the presence of the necrotic liver exerts local adverse effects. The exact mechanisms as well as the localization of the proposed tissue lysis can not be deduced from our experiment.

To summarize, we observed during acute severe hyperammonemia an expected rise in arterial glutamine levels. In contrast to suggestions in the literature, this rise could not be explained by enhanced skeletal muscle glutamine release. Our hypothesis is that a continuous, although minimal glutamine release from skeletal muscle combined with a decreased glutamine consumption in the splanchnic area (both liver and intestine), could explain the rise in glutamine levels. Also, glutamine production by other glutamine synthetase containing organs, like brain, lung or heart (44) and/or decreased consumption by kidney or immune system could contribute to the observed rise in arterial glutamine concentration.

We conclude that the proposed mechanisms of muscular ammonia detoxification are not operative after 24 hours PCS and during acute liver ischemia, and the results cast doubt that this 'hypothesis' is generally applicable to all liver insufficiency-induced hyperammonemic states. Our results suggest that the role of skeletal muscle in the detoxification of ammonia during severe acute hyperammonemia induced by acute liver ischemia in the rat is small. Changes in intestinal metabolism may play an important role in the alterations in amino acid and ammonia metabolism during hyperammonemia.

\section{REFERENCES}

1. Zieve L. Pathogenesis of hepatic encephalopathy. Metab Brain Dis 1987;2:147-165.

2. Schalm SW, van der Mey T. Hyperammonemic coma after hepatectomy in germ-free rats. Gastroenterology 1979;77:231-234.

3. Lockwood AH, McDonald JM, Reiman RE, Gelbard AS, Laughlin JS, Duffy TE, Plum F. The dynamics of ammonia metabolism in man. Effects of liver disease and hyperammonemia. J Clin Invest 1979:63:449-460.

4. Deutz NEP, Chamuleau RAFM, de Graaf AA, Bovée WMMJ, de Beer R. In vivo ${ }^{3}$ P NMR spectroscopy of the rat cerebral cortex during acute hepatic encephalopathy. NMR Biomed 1988; 1:101-106.

5. Deutz NEP. Pathophysiological aspects of acute hepatic encephalopathy in the rat. An electroencephalographic, biochemical and in vivo brain NMR spectroscopic study. Thesis, University of Amsterdam, The Netherlands, 1988.

6. Ganda OP, Ruderman NB. Muscle nitrogen metabolism in chronic hepatic insufficiency. Metabolism 1976;25:427-435.

7. Bessman SP, Bradley JE. Uptake of ammonia by muscle. Its implications in ammoniagenic coma. $\mathrm{N}$ Eng J Med 1955;253:1 143-1 147.

8. Webster LT, Gabuzda GJ. Ammonium uptake by the extremities and brain in hepatic coma. I Clin Invest 1958;37:414-424.

9. Duda GD, Handler P. Kinetics of ammonia metabolism in vivo. J Biol Chem 1958;232:303-314.

10. Lund P, Watford M. Glutamine as a precursor of urea. In: Grisolia S, Baguena R, Mayor F, eds. The urea cycle. New York: John Wiley and Sons, 1976:479-488.

11. Schröck H, Goldstein L. Interorgan relationships for glutamine metabolism in normal and acidotic rats. Am J Physiol 1981;240:E519-E525.

12. Rosado A, Flores G, Mora J, Soberón G. Distribution of an ammonia load in the normal rat. Am J Physiol 1962;203:37-42.

13. Windmueller HG. Glutamine utilization by the small intestine. Adv Enzymol 1982;53:210-237. 
14. Plauth M, Graser TA, Vieillard-Baron D, Bauder D, Fürst P, Hartmann F. Influence of paromomycin and lactulose on the endogenous ammonia production in the isolated perfused rat intestine correlation of glutamine consumption and ammonia formation. In: Soeters PB, Wilson JHP, Meijer AJ, Holm E, eds. Advances in ammonia metabolism and hepatic encephalopathy. Amsterdam: Excerpta Medica, 1988:170-176.

15. Weber FL, Friedman DW, Fresard KM. Ammonia production from intraluminal amino acids in canine jejunum. Am J Physiol 1988;254:G264-G268.

16. Weber FL, Veach GL. The importance of the small intestine in gut ammonium production in the fasting dog. Gastroenterology 1979;77:235-240.

17. Windmuelier HG, Spaeth AE. Intestinal metabolism of glutamine and glutamate from the lumen as compared to glutamine from blood. Arch Biochem Biophys 1975;171:662-672.

18. Windmueller $\mathrm{HG}$, Spaeth $\mathrm{AE}$. Uptake and metabolism of plasma glutamine by the small intestine. $\mathrm{J}$ Biol Chem 1974;249:5070-5079.

19. Chang TW, Goldberg AL. The metabolic fates of amino acids and the formation of glutamine in skeletal muscle. J Biol Chem 1978;253:3685-3695.

20. Wahren J. Extra-hepatic ammonia metabolism. In: Soeters PB, Wilson JHP, Meijer AJ, Holm E, eds. Advances in ammonia metabolism and hepatic encephalopathy. Amsterdam: Excerpta Medica, 1988:121-129.

21. Newsholme EA, Leech AR. Biochemistry for the Medical Sciences. New York: John Wiley \& Sons, 1983.

22. Cooper AJL, Plum F. Biochemistry and physiology of brain ammonia. Physiol Rev 1987;67:440-519.

23. Imler M, Chabrier $\mathrm{G}$, Simon $\mathrm{C}$, Schlienger $\mathrm{J}$. Intestinal ammonium production in the rat: the role of the colon, small intestine, and circulating glutamine. Res Exp Med 1988;188:1-7.

24. Funovics JM, Cummings MG, Shuman L, James JH, Fischer JE. An improved nonsuture method for portacaval anastomosis in the rat. Surgery 1975;77:661-664.

25 Bosman DK, Deutz NEP, de Graaf A, van der Hulst RWN, van Eijk HMH, Bovée WMMJ, Maas MAW, Jörning GGA, Chamuleau RAFM. Changes in brain metabolism during hyperammonemia and acute liver failure: results of a comparative 'H-NMR spectroscopy and biochemical investigation. Hepatology 1990:12:281-290.

26. Bosman DK, de Haan JG, Smit J, Jöming GGA, Maas MAW, Chamuleau RAFM. Metabolic activity of microcarrier attached liver cells after intraperitoneal transplantation during severe liver insufficiency in the rat. J Hepatol 1989:9:49-58.

27. Drapanas T, McMenamy RH, Adler WJ, Vang JO. Intermediary metabolism following hepatectomy in dogs. Ann Surg 1965; 162:621-633.

28. Welboume TC, Phromphetcharat V, Givens G, Joshi S. Regulation of interorganal glutamine flow in metabolic acidosis. Am J Physiol 1986;250:E457-E463.

29. Phromphetcharat V, Welbourne TC. Renal glutamine extraction and gut/liver interaction in glutamine homeostasis. Contrib Nephrol 1985;47:9-14.

30 Welboume TC. Effect of metabolic acidosis on hindquarter glutamine and alanine release. Metabolism 1986;35:614-618.

31. Buttrose M, McKellar D, Welboume TC. Gut-liver interaction in glutamine homeostasis: portal ammonia role in uptake and metabolism. Am J Physiol 1987;252:E746-E750.

32. Souba WW, Herskowotz K, Klimberg VS, Salloum RM, Plumley DA, Flynn TC, Copeland EM. The effects of sepsis and endutoxemia on gut glutamine metabolism. Ann Surg 1990;211:543-551.

33. Ardawi MSM. Skeletal muscle glutamine production in thermally injured rats. Clin Sci 1988;74:165-172.

34. Ardawi MSM, Jamal YS. Glutamine metabolism in skeletal muscle of glucocorticoid-treated rats. Clin Sci 1990;79:139-147.

35. Rudeman NB, Houghton CRS. Hems R. Evaluation of the isolated perfused rat hindquarter for the study of muscle metibolism. Biochem J 1971;124:639-651.

36. Souba WW, Strebel FR, Bull JM, Copeland EM, Teagtmeyer H, Cleary K. Interorgan glutamine metabolism in the tumor-bearing rat. J Surg Res 1988;44:720-726.

37. Wollenberger A, Ristau O, Schoffa G. Eine einfache Technik der extrem schnellen Abkühlung größerer Gewebestücke. Pflügers Arch 1960;270:399-412.

38. Janssen MA, van Berlo CLH, van Leeuwen PAM, Soeters PB. The determination of ammonia in plasma and whole blood. In: Soeters PB, Wilson JHP, Meijer AJ, Holm E, eds. Advances in ammonia metabolism and hepatic encephalopathy. Amsterdam: Excerpta Medica, 1988:587-592.

39. van Eijk HMH, van der Heijden MAH, van Berlo CLH, Soeters PB. Fully automated liquid-chromatographic determination of amino acids. Clin Chem 1988;34:2510-2513. 
40. Brun C. A rapid method for the determination of para-aminohippuric acid in kidney function tests. $J$ Lab Clin Med 1951;37:955-958.

41. Katz ML, Bergman EN. Simultaneous measurements of hepatic and poral venous blood flow in the sheep and dog. Am J Physiol 1969;216:946-952.

42. Norusis MJ. SPSS/PC+ V2.0 BASE MANUAL for the IBM PC/XT/AT and PS/2. SPSS Inc, Chicigo, USA, 1988.

43. Rémésy C, Demigné C, Aufrère J. Inter-organ relationships between glucose, lactate and amino acids in rats fed on high-carbohydrate or high-protein diets. Biochem J 1978;1 70:321-329.

44. Lund P. A radiochemical assay for glutamine synthetase, and activity of the enzyme in rat tissues. Biochem J 1970; 1 18:35-39.

45. Souba WW, Smith RJ, Wilmore DW. Glutamine metabolism by the intestinal tract. JPEN 1985;9:608-617.

46. Caster WO, Poncelet J, Simon AB, Armstrong WD. Tissue weights of the rat. I. Normal values determined by dissection and chemical methods. Proc Soc Exp Biol Med 1956;91:122-126.

47. Kimura RE, LaPine TR, Gooch WM. Portal venous and aortic glucose and lactate changes in a chronically catheterized rat. Pediatr Res 1988;23:235-240.

48. Kimura RE, LaPine TR, Johnston J, Ilich JZ. The effect of fasting on rat portal venous and aortic blood glucose, lactate, alanine, and glutamine. Pediatr Res 1988;23:241-244.

49. Mans AM, Saunders SJ, Kirsch RE, Biebuyck JF. Correlation of plasma and brain amino acid and putative neurotransmitter alterations during acute hepatic coma in the rat. J Neurochem 1979;32:285292.

50. Chamuleau RAFM, Deutz NEP, de Haan JG, van Gool J. Correlation between electroencephalographic and biochemical indices in acute hepatic encephalopathy in rats. J Hepatol 1987;4:299-306.

51. James JH, Herlin PM, Edwards L, Nachbauer CA, Fischer JE. Effect of infusing the branched-chain amino acids on concentrations of amino acids in plasma and brain and on brain catecholamines after total hepatectomy in the rat. Life Sci 1982;30:1361-1368.

52. Albrecht J, Hilgier W. Arginine in thioacetamide-induced hepatogenic encephalopathy in rats: activation of enzymes of arginine metabolism to glutamate. Acta Neurol Scand 1986;73:498-501.

53. Hills AG, Reid EL, Ker WD. Circulatory transport of L-glutamine in fasted mammals: cellular sources of urine ammonia. Am J Physiol 1967;223:1470-1476.

54. Fine $\mathrm{A}$. The effects of ammonia infusion on ammonia and glutamine metabolism by liver and muscle in the normal dog. Contrib Nephrol 1985;47:1-8.

55. Ruderman NB, Lund P. Amino acid metabolism in skeletal muscle. Regulation of glutamine and alanine release in the perfused rat hindquarter. Isr J Med Sci 1972;8:295-302.

56. Reijven PLM, Deutz NEP, Soters PB. Net ammonia (AMM) uptake of the pig hindquarter (HQ) exceeds by far net glutamine (GLN) efflux. J Hepatol 1990; 10:S21 (Abstract).

57. Rennie MJ, Hundal HS, Babij P, MacLennan P, Taylor PM, Watt PW. Characteristics of a glutamine carrier in skeletal muscle have important consequences for nitrogen loss in injury, infection, and chronic disease. Lancet 1986;ii:1008-1011.

58. Smith RJ, Larson S, Stred SE, Durschlag RP. Regulation of glutamine synthetase and glutaminase activities in cultured skeletal muscle cells. J Cell Physiol 1984;120:197-203.

59. Goldberg AL, Chang TW. Regulation and significance of amino acid metabolism in skeletal muscle. Fed Proc 1978;37:2301-2307.

60. Durschlag RP, Smith RJ. Regulation of glutamine production by skeletal muscle cells in culture. Am J Physiol 1985;248:C442-C448.

61. Kominz DR, Hough A, Symonds P, Laki K. The amino acid composition of actin, myosin, tropomyosin and the meromyosins. Arch Biochem Biophys 1954;50: 148-159.

62. Thomas RC. Experimental displacement of intracellular $\mathrm{pH}$ and the mechanism of its subsequent recovery. J Physiol (London) 1984;354:3P-22P.

63. Albina JE, Shearer J, Mastrofrancesco B, Caldwell MD. Amino acid metabolism after lambdacarrageenan injury to rat skeletal muscle. Am J Physiol 1986;250:E24-E30.

64. Kadowaki M, Harada N, Takahashi S, Noguchi T, Naito H. Differential regulation of the degradation of myofibrillar and total proteins in skeletal muscle of rats: Effects of streptozotocin-induced diabetes, dietary protein and starvation. J Nutr 1989;119:471-477.

65. Plauth M, Raible A, Vieillard-Baron D, Bauder D, Kremer I, Hartmann F. Effect of glucose and ammonia on glutamine (GLN) metabolism in the isolated perfused rat intestine. Gastroenterology 1991;100:A240 (abstract). 
66. Windmueller HG, Spaeth AE. Identification of ketone bodies and glutamine as the major respiratory fuels in vivu for postabsorptive rat small intestine. J Biol Chem 1978;253:69-76.

67. Hammarqvist F, Wernennan J, Ali R, von der Decken A, Vinnars E. Addition of glutamine to total parenteral nutrition after elective surgery spares free glutamine in muscle, counteracts the fall in muscle protein synthesis, and improves nitrogen balance. Ann Surg 1989;209:455-461.

68. Wilmore DW, Smith RJ, O'Dwyer ST, Jacobs DO, Ziegler TR, Wang XD. The gut: A central organ after surgical stress. Surgery 1988;104:917-923.

69. O'Dwyer ST, Smith RJ, Hwang TL, Wilmore DW. Maintenance of small bowel mucosa with glutamine-enriched parenteral nutrition. JPEN 1989;13:579-585.

70. Jacobs DO, Evans DA, Mealy K, O'Dwyer ST, Smith RJ, Wilmore DW. Combined effects of glutamine and epidermal growth factor on the rat intestine. Surgery 1988; 104:358-364.

71. Baskerville A, Hambleton P, Benbough JE. Pathological features of glutaminase toxicity. $\mathrm{Br} J \mathrm{Exp}$ Pathol 1980;61:132-138.

72. Prior RL, Topping DC, Visek WJ. Metabolism of isolated chick small intestinal cells. Effects of ammonia and various salts. Biochemistry 1974; 13:178-183.

73. Zimber A, Visek WJ. Effect of urease injections on DNA synthesis in mice. Am J Physiol 1972;223:1004-1008. 


\title{
Chapter 6
}

\section{Cerebral cortex during acute liver failure}

\author{
A. Blood flow measurement ${ }^{1}$
}

Published as: C.H.C. Dejong, N.E.P. Deutz, P.B. Soeters. A simple method for repeated in vivo cerebral contex flux measurements in rats. Lah Anim Sci 1992,42:280-285.

\begin{abstract}
A new application of an indicator dilution technique, using non-radioactive para-aminohippuric acid, is described for superior sagittal sinus blood flow determination in rats. Superior sagittal sinus blood flow, mainly representing cerebral cortex blood flow, amounted to $541 \pm$ $49 \mu \mathrm{L} \min ^{-1}$ in ketamine anesthetized, but otherwise normal rats breathing room air. Increasing arterial $\mathrm{pCO}_{2}$ enhanced superior sagittal sinus blood flow ( $\mathrm{P}<0.01$ ), providing evidence that this method correctly measures cerebral blood flow. The respiratory quotient was 1.1 and the cerebral cortex metabolic rate of oxygen consumption was \pm 1.45 $\mu \mathrm{mol} \mathrm{min}^{-1}$. Cerebral cortex ammonia uptake was not significantly different from zero and of the amino acid fluxes only alanine differed from zero $(\mathrm{P}<0.05)$. Flux measurements are crucial in studies of healthy or altered organ metabolism in both experimental animals and postoperative surgical patients. We devised a simple and economical method of repeated cerebral cortex flux measurement in rats that is a potentially valuable tool in metabolic studies of the cerebral cortex.
\end{abstract}

\footnotetext{
'This chapter is an exact copy of the original publication and consequently does not present all data or steps of the surgical procedures from these experiments. Additional data or pictures are available an special request.
} 


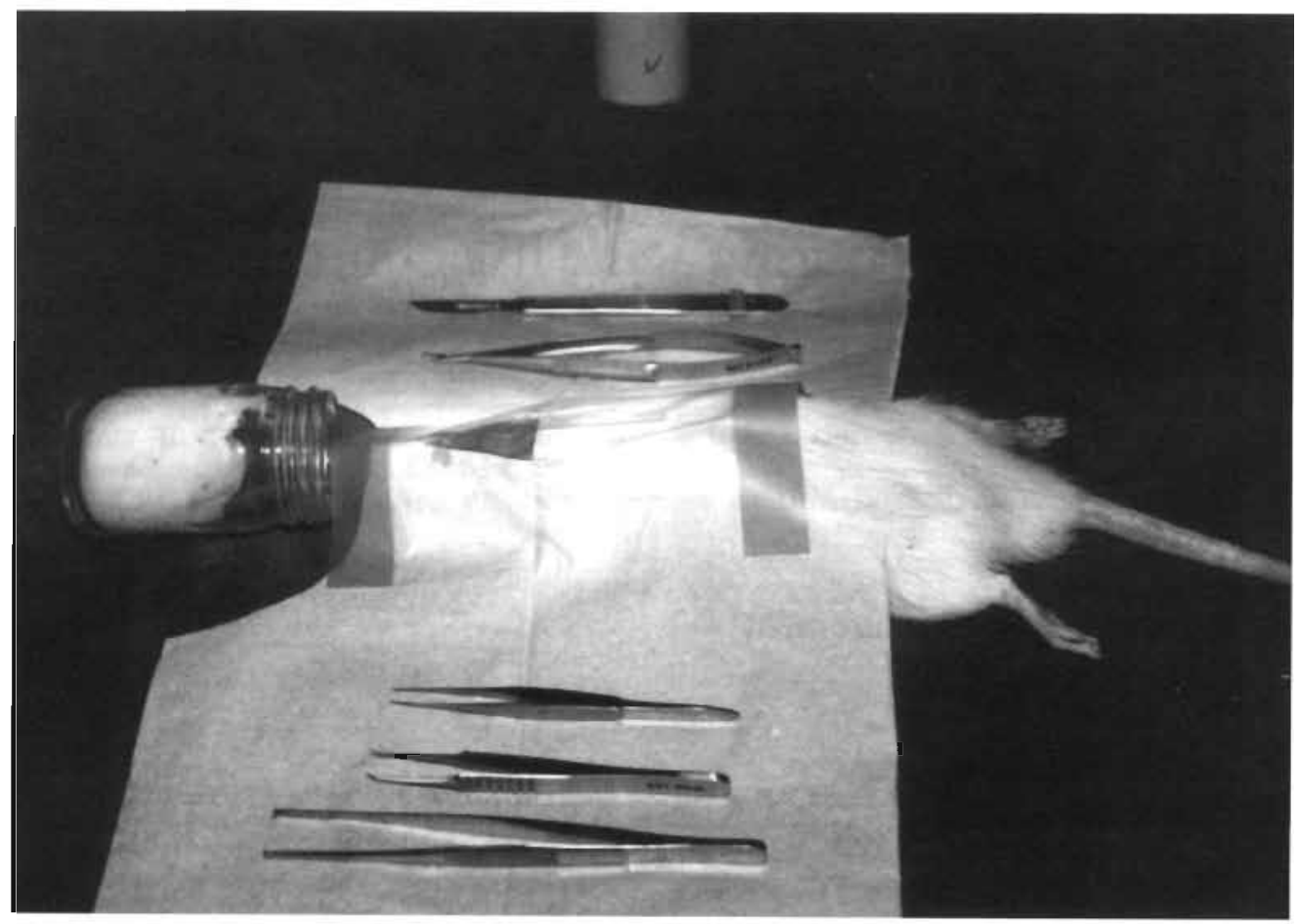

Figure 1. Wound site preparation. The head was shaved, cleaned with Savion and disinfected with tincture of iodine ( $2 \%$ w/N). Next, the surgical field was covered with sterile drapes and self-adhesives (Op-Site), as shown.

\section{INTRODUCTION}

Brain flux measurements are complicated by the relative inaccessibility of cerebral blood vessels, hampering both blood sampling and blood flow measurement. Several methods have been used to measure cerebral blood flow in rats. Most techniques are based on using radioactive isotopes either given intravenously $(1-4)$ or by inhalation $(5,6)$. Consequently these methods are expensive, labor-intensive (5), relatively complex $(1,4,5)$ and, despite recent refinements $(1,4-6)$ require relatively large amounts of blood $(5,6)$, making repeated measurements in small laboratory animals difficult. Moreover, most tracer techniques as well as the microsphere technique, permit estimation of cerebral blood flow only after an animal is dead $(1,2)$.

Hence, there is no good method that allows repeated in vivo measurement of cerebral cortex blood flow in rats. Because we were interested in cerebral cortex metabolism during various metabolic disorders in rats, we developed a method of making serial, simultaneous in vivo measurements of cerebral cortex blood flow and venous-arterial concentration differences, thereby enabling calculation of metabolite fluxes across the cerebral cortex in rats.

The method described is based on an indicator dilution technique using non-radioactive para-aminohippuric acid ( $\mathrm{PAH})$ as an indicator. It extends the applicability of this economi- 


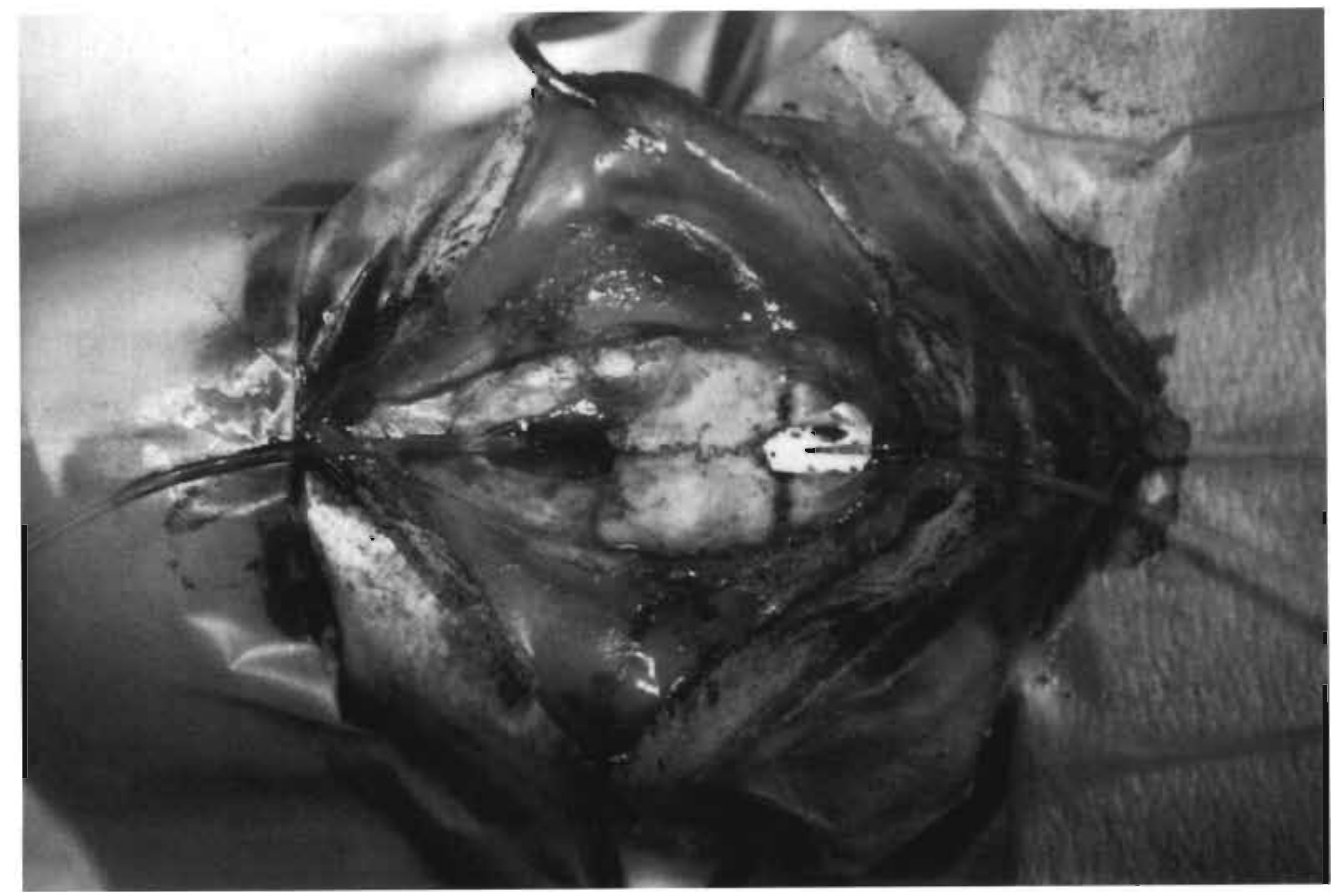

Figure 2. Insertion of the anterior catheter. The bone has been milled away, exposing the superior sagittal sinus anterior to the bregma and the confluence of sinuses posterior to the lambdoid suture. The posterior catheter has already been inserled and fixed with cyano-acrylate adhesive and a suture through the periost at the occipital notch.

cal indicator dilution method, commonly used in flux measurements in several other organs (7-11) to the cerebral cortex of rats.

\section{MATERIALS AND METHODS}

Animals Male SPF Wistar rats (250 to $300 \mathrm{~g}$, random bred Wistar/CPB:WU/Bor, Winkelmann, Borchen, F.R.G.), housed under standard conditions (12/12 hour light/dark cycle, standard laboratory rat ration and water ad libitum), were used in all experiments. Drinking water was acidified to $\mathrm{pH}=3$ to prevent the growth of micro-organisms (standard procedure in our institute). Rats were maintained and cared for in accordance with the Guide for the Care and Use of Laboratory Animals.

Catheters Sterile composite catheters were constructed of PE 50 tubing, snugly fitted over a segment of PE 10 tubing (Intramedic ${ }^{\circledR}$ Polyethylene Tubing PE-50: $0.58 \mathrm{~mm} \mathrm{ID,} 0.96 \mathrm{~mm}$ OD; PE-10: $0.30 \mathrm{~mm}$ ID, $0.58 \mathrm{~mm}$ OD, Clay Adams, Parsippany, NJ, USA). In all instances the vessels were cannulated with the PE 10 segments of these catheters. Catheters were all heparinized (50 IU $\mathrm{mL}^{-1} 0.9 \% \mathrm{NaCl}$; Heparine, Leo Pharmaceutische Produkten B.V., Emmen, The Netherlands) and had blunt tips. 


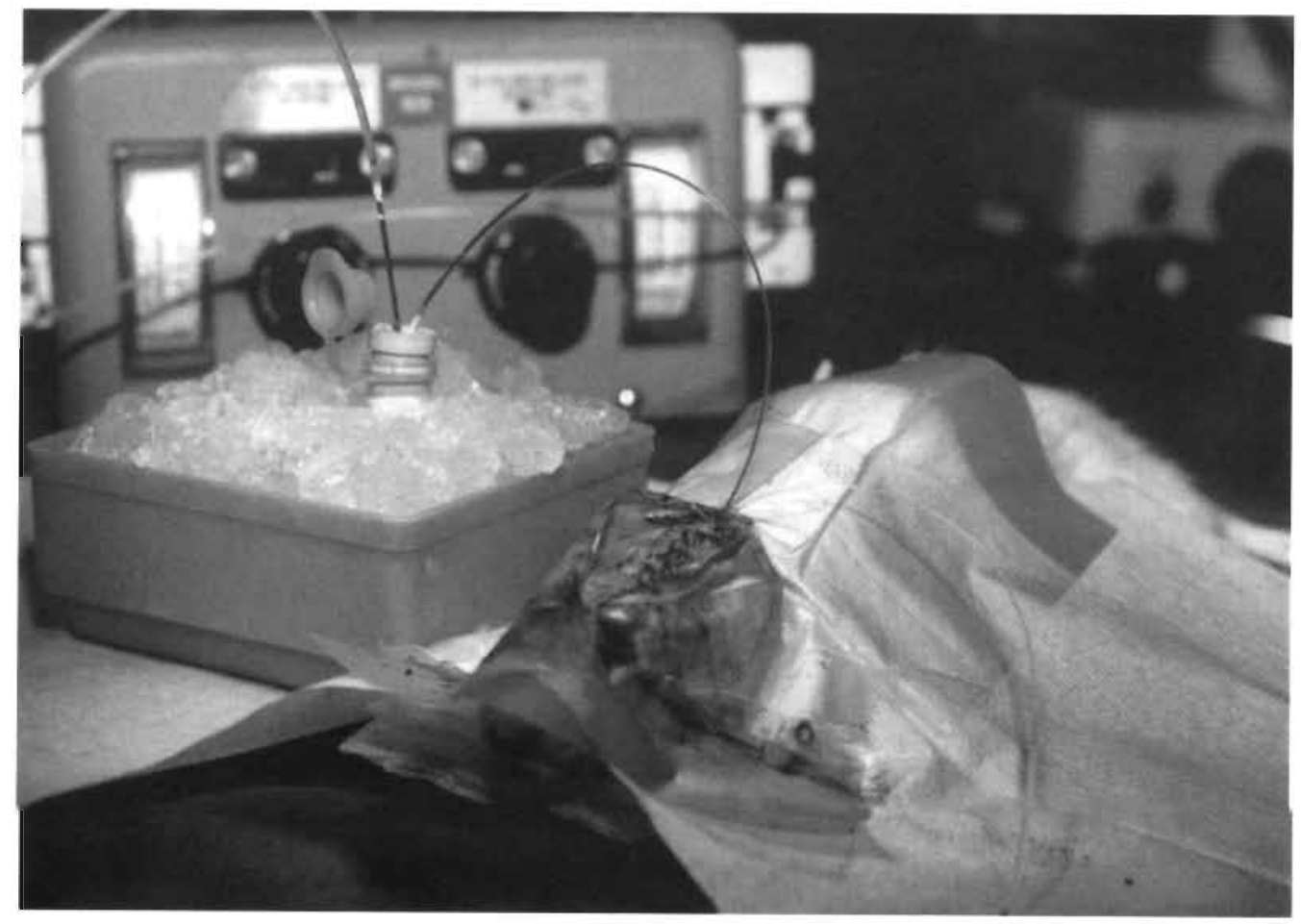

Figure 3. Blood sampling after skin closure. Underpressure is created in a cup (placed on ice), to which the confluence of sinuses catheter is connected.

Surgical procedures Using aseptic techniques, catheter implantations were performed on ether anesthetized rats. Sampling procedures were performed on ketamine anesthetized rats (75 $\mathrm{mg} \cdot \mathrm{kg}$ body weight ${ }^{-1}$; Ketamine $\mathrm{HCl}$, Nimatek ${ }^{\circledR}$, AUV Cuijck, The Netherlands). Animal heating boards and heating lamps provided constant core temperatures $\left(37 \pm 1^{\circ} \mathrm{C}\right)$.

After shaving and cleaning with Savlon (Savlon ${ }^{\otimes}: 1.5 \%(\mathrm{w} / \mathrm{v})$ chloorhexidine-digluconate and $15 \%(\mathrm{w} / \mathrm{v})$ cetrimide, 100 times diluted in distilled water, ICI-Farma, Rotterdam, The Netherlands), the surgical field was iodinated (tincture of iodine, $2 \% \mathrm{w} / \mathrm{v} ;$ AUV Cuijck, The Netherlands.) and covered with commercially available sterile drapes and sterile self-

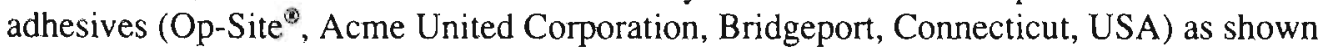
in Figure 1. Using a sagittal incision, the skull was exposed from the midpoint between the eyes to the interscapular region. Using a commercially available minidrill (Blitz ${ }^{\circledR}$, Stichling, FRG) with sterile bit-holder and bit, the diploe was milled away over a distance of $0.5 \mathrm{~cm}$ posterior to the lambdoid suture and $1.0 \mathrm{~cm}$ anterior to the bregma (Figure 2) to expose the confluence of sinuses and the anterior part of the superior sagittal sinus (SSS), respectively. The confluence of sinuses was then cannulated using a modification of the technique described by Urban for portal vein cannulation (12). A 25 -gauge needle was inserted into the confluence of sinuses, slowly withdrawn, and as the $45^{\circ}$ bevel of the needle reached the vessel wall, it was gently lifted. At this time, the unbevelled PE 10 catheter was gently 


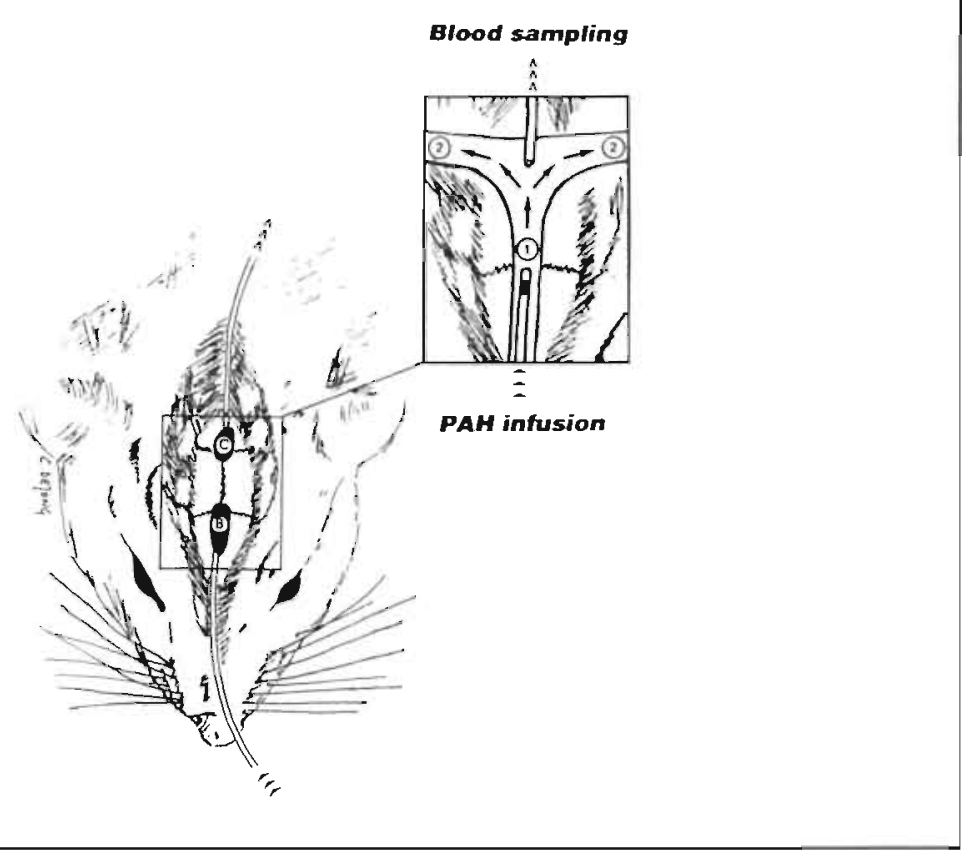

Figure 4. The superior sagittal sinus (1) carries the blood from the bregma $(B)$ to the confluence of sinuses (C) $(25,26)$ as indicated in the schematic drawing (upper right). $\rightarrow=\mathrm{PAH}$ infusion; $\gg=$ blood sampling; $\rightarrow \rightarrow \rightarrow=$ direction of blood flow. From the confluence, blood is drained by the transverse sinuses (2) into the internal maxillary vein, which finally joins the external jugular vein, contributing to the subclavian vein (25).

inserted $3.5 \mathrm{~mm}$ into the confluence by sliding it along the bevel of the 25 gauge needle, simultaneously withdrawing the latter. Constant catheter position was attained by marking the catheter at $3.5 \mathrm{~mm}$ from its tip; the catheter was inserted until this mark reached the vessel wall. Next a PE 10 catheter was inserted into the anterior part of the superior sagittal sinus in the same way as described above, the tip being positioned under the bregma. The tip of this catheter was also marked with ink to allow correct positioning at the bregma under visual control. The anterior SSS catheter was used for PAH infusion for flow determinations, the confluence of sinuses catheter for blood sampling (Figures 2, 3 and 4).

The catheters were cemented in place with high viscosity cyanoacrylate rapid binding adhesive and the posterior catheter was secured with a silk 7-0 periost suture at the occipital notch (Figure 2). The catheters were then coded, sealed and positioned subcutaneously in the neck. For arterial blood sampling, a sterile catheter was inserted via the femoral artery into the abdominal aorta, sealed, coded, tunneled and placed subcutaneously in the neck. The total surgical procedure was completed within 45 minutes.

Sampling procedures Sampling procedures were performed 24 hours after catheter implantation. An isomolaric, pH adjusted, $50 \mathrm{mM}$ PAH solution (para-aminohippuric acid, Sigma Chemical Co., St. Louis, MO, USA) was infused in the anterior sagittal sinus catheter 
at a rate of $30 \mu \mathrm{L} \min ^{-1}$ (Minipuls 2 pump, Gilson France SA, Villiers le Bel, France) including a 20 -minute equilibration period before blood sampling. In prior experiments, the 20-minute equilibration period provided steady state arterial PAH concentrations (data not shown), as described previously $(13,14)$.

Blood for blood gas analysis was collected under strictly anaerobic conditions in heparinized syringes at a rate of $50 \mu \mathrm{L} \mathrm{min}^{-1}$. Blood samples for PAH, ammonia and amino acids were collected semi-automatically on ice in heparinized cups (Microvette ${ }^{\circledR}$ LH CB1000, Sarstedt, Nümbrecht, FRG) sealed by rubber stoppers, perforated by a 25 and an 18 gauge needle. The PE 50 ends of the arterial or venous catheters were connected to the 25 gauge needle. For venous sampling, the 18 gauge needle was connected by means of PE 100 tubing to a pump (Model 925 Holter $^{*}$, Extracorporeal SA., Brussels, Belgium) creating underpressure in the cup (Figure 3), providing a sampling rate of $50 \mu \mathrm{L} \mathrm{min}{ }^{-1}$. Arterial samples were collected in a freely flowing manner. The blood samples were kept on ice during transport to the laboratory for further processing before storage at $-70^{\circ} \mathrm{C}$.

Calculations Blood flow was calculated by using the formula introduced by Katz and Bergmann (15) based on the Fick principle for indicator dilution methods:

$$
F_{S S S}=\frac{1}{[P A H]_{C}-[P A H]_{A}}
$$

In this formula, $\mathrm{F}_{S S S}$ is SSS blood flow $\left(\mathrm{mL} \mathrm{min}^{-1}\right), I$ is the infused PAH $\left(\mu \mathrm{mol} \mathrm{min}^{-1}\right)$, and $[\mathrm{PAH}]_{\mathrm{C}(\mathrm{A})}$ is the concentration of PAH $\left(\mu \mathrm{mol} \mathrm{mL}^{-1}\right)$ in the confluence of sinuses $(\mathrm{C})$ and arterial (A) blood, respectively.

Basal blood flow and the influence of $\mathrm{pCO}_{2}$ In a first study, basal SSS blood flow was determined in anesthetized rats $(n=6)$ spontaneously breathing a room air mixture administered through a nose cone. After a 20 -minute equilibration period, $300 \mu \mathrm{L}$ of arterial blood was collected in 3 to 5 minutes for blood gas analysis and hemoglobin determination. Next, $200 \mu \mathrm{L}$ of arterial and SSS blood were simultaneously collected for PAH concentration and hematocrit determinations. Mean arterial PAH concentration was $141 \pm 22 \mu \mathrm{M}$ and the mean $[\mathrm{PAH}]_{\mathrm{C}}-[\mathrm{PAH}]_{\mathrm{A}}$ difference at steady state was $4401 \pm 392 \mu \mathrm{M}$. Arterial $\mathrm{pCO}_{2}$ values between approximately 4.5 and $5.5 \mathrm{kPa}(35-45 \mathrm{mmHg})$ were considered to be within the normal range $(16,17)$.

To study the effect of increasing arterial $\mathrm{pCO}_{2}$ on cerebral cortex blood flow in these rats, arterial $\mathrm{pCO}_{2}$ was increased by selectively adding $\mathrm{CO}_{2}$ to the inspired room air mixture to produce either normocapnia (arterial $\mathrm{pCO}_{2}$ between 4.5 and $5.5 \mathrm{kPa}$ ), moderate hypercapnia (arterial $\mathrm{pCO}_{2}$ between 5.5 and $7.5 \mathrm{kPa}$ ) or severe hypercapnia (arterial $\mathrm{pCO}_{2}$ between 7.5 and $12.0 \mathrm{kPa}$ ). Blood was collected repeatedly at increasing $\mathrm{pCO}_{2}$ 's in the same rat. Blood transfusions were administered to provide constant hemoglobin levels.

Fluxes and respiratory quotient $(R Q)$ In a second series of five anesthetized normal rats, femoral artery and SSS blood $(300 \mu \mathrm{L})$ were obtained as described above to determine the $\mathrm{RQ}$ and cerebral cortex metabolic rate of oxygen consumption $(\mathrm{cCMRO})$. RQ was calculated as the venous-arterial (V-A) difference in total $\mathrm{CO}_{2}$ content divided by the $\mathrm{V}-\mathrm{A}$ difference in $\mathrm{O}_{2}$ content. The $\mathrm{CCMRO}_{2}$ was calculated by multiplying the venous-arterial difference in oxygen content with the SSS blood flow $(6,18)$. 
Next, PAH infusion was begun and after a 20 -minute equilibration period, blood was sampled for blood flow and amino acid and ammonia determinations. Ammonia and amino acids fluxes were calculated as mean plasma flow times plasma $\mathrm{V}$-A differences.

Reproducibility of blood flow measurements The repeatability of superior sagittal sinus blood flow measurements was studied in experiments reported previously (19). In this series of 32 rats with acute liver ischemia (as a model of acute hyperammonemia (14)) or portacaval shunts, blood flow was measured using the method described and determined to be statistically identical in all groups. Therefore, since blood flow in this study had been measured twice at either 2 -hour or 4hour intervals, these data were used to estimate repeatability. The second blood flow measurement was then expressed as a percentage of the first (see 'Results').

Blood processing Centrifugation was performed at $4^{\circ} \mathrm{C}$ for 5 minutes at $8,500 \mathrm{~g}$. Hematocrit was obtained with a microfuge. For PAH determinations $100 \mu \mathrm{L}$ of whole blood was deproteinized with $400 \mu \mathrm{L}$ of $10 \%$ trichloracetic acid, thoroughly mixed, allowed to stand for deproteinization $\left(4^{\circ} \mathrm{C}, 30\right.$ minutes) and centrifuged after which the supernatant was stored at $-70^{\circ} \mathrm{C}$ until further analysis. Plasma for ammonia and amino acid determination was obtained by whole blood centrifugation in heparinized cups.

Biochemical analyses For PAH-determination the method according to Brun (20) was adapted for small volumes. For spectrophotometric (Cobas Bio, Roche, Basle, Switzerland) PAH-measurement at $465 \mathrm{~nm}, 100 \mu \mathrm{L}$ of Ehrlich's reagent (1 g p-dimethylaminobenzaldehyde (Para-dimethylaminobenzaldehyde, Sigma D 2004, St. Louis, MO, USA), $60 \mathrm{~mL}$ alcohol $93 \%, 4 \mathrm{~mL} \mathrm{HCl} 2 \mathrm{~N}$, distilled water added up to a final total volume of $100 \mathrm{~mL}$ ) was added to a $50 \mu \mathrm{L}$ sample and $30 \mu \mathrm{L}$ of distilled water. Amino acids were determined in sulfosalicylic acid (5\% w/v) deproteinized plasma by standard HPLC techniques (2l). Plasma ammonia was measured by standard enzymatic methods (22). Blood gas analysis and hemoglobin determination was performed immediately after sampling on a blood gas analyzer (Acid Base Laboratory, ABL3, Radiometer, Copenhagen, Denmark). The values are corrected for temperature (to $37^{\circ} \mathrm{C}$ ).

Statistical analysis Results are presented as means \pm 1 SEM. Levels of significance were set at $\mathrm{P}<0.05$ unless stated otherwise. For statistical analysis (23) the analysis of variance, repeated measurement design (MANOVA) or Wilcoxon test were used, when appropriate. 
Table 1. Cerebral cortex fluxes

\begin{tabular}{|c|c|c|c|c|c|c|}
\hline $\mathrm{CCMRO}_{2}$ & -1452 & $\pm 267+$ & TAU & 1.4 & \pm & 0.7 \\
\hline Amrn & -1.7 & $\pm \quad 1.0$ & ARG & -1.9 & \pm & 1.4 \\
\hline GLU & -0.6 & $\pm \quad 1.5$ & TYR & 1.1 & \pm & 1.2 \\
\hline ASN & 0.1 & $\pm \quad 0.6$ & VAL & 1.6 & \pm & 0.6 \\
\hline SER & -1.5 & $\pm \quad 1.8$ & MET & -0.1 & \pm & 0.2 \\
\hline GLN & -2.9 & $\pm \quad 7.5$ & ILE & 0.2 & \pm & 0.3 \\
\hline GLYY & -4.2 & $\pm \quad 4.8$ & PHE & 0.7 & \pm & 0.5 \\
\hline THR & -2.0 & $\pm \quad 1.6$ & TRP & 0.2 & \pm & 0.5 \\
\hline HIS & -0.2 & $\pm \quad 0.4$ & LEU & -0.4 & \pm & 1.3 \\
\hline CIT & 0.0 & $\pm \quad 0.6$ & LYS & -7.6 & \pm & 6.0 \\
\hline ALA & 6.8 & $\pm \quad 3.7^{+}$ & $B C A A$ & 1.4 & \pm & 2.1 \\
\hline$\alpha A N$ & -9.3 & \pm 18.8 & & & & \\
\hline
\end{tabular}

Values are expressed as $n$ mol min $^{-1}$ (mean $\pm S E M, n=5$ ), negative values reflect net uptake. $\alpha A N=\alpha$-Amino Nitrogen. Difference from zero (t) was tested by the Wilcoxan test.

\section{RESULTS}

Basal blood flow and the influence of $p \mathrm{CO}_{2}$ SSS blood flow in ketamine-anesthetized rats with arterial $\mathrm{pCO}_{2}$ values of $5.52 \pm 0.23 \mathrm{kPa}$, breathing room air was $541 \pm 49 \mu \mathrm{L} \mathrm{min}^{-1}$. Increasing arterial $\mathrm{pCO}_{2}$ induced an increase in $\mathrm{SSS}$ blood flow $(\mathrm{P}<0.01)$ (Figure 5) by approximately $2 \%$ per $\mathrm{mm} \mathrm{Hg}$ ( $15 \%$ per $\mathrm{kPa})$.

Fluxes (Table 1 ) and $R Q$ In the rats used for fluxes and RQ determinations, SSS blood flow was $747 \pm 122 \mu \mathrm{L} \mathrm{min}^{-1}$ (arterial $\mathrm{pCO}_{2} 5.94 \pm 0.29 \mathrm{kPa}$ ), plasma flow was $488 \pm 81$ $\mu \mathrm{L} \cdot \mathrm{min}^{-1}$. Cerebral cortex ammonia uptake was not significantly different from zero. Of the amino acid fluxes, only alanine release was significantly different from zero. RQ was $1.11 \pm$ $0.25, \mathrm{cCMRO}_{2}$ was $1.97 \mu \mathrm{mol} \cdot \mathrm{mL}^{-1} * 0.747 \mathrm{~mL} \mathrm{~min}^{-1}=1.45 \mu \mathrm{mol} \cdot \mathrm{min}^{-1}$.

Repeatability In 32 rats with acute liver ischemia or portacaval shunting $(14,19)$, two consecutive measurements were performed at either 2 - or 4 -hour intervals. The second measurement was on the average $97 \pm 7 \%$ of the first measurement.

\section{DISCUSSION}

The method presented enables simultaneous flow and $\mathrm{V}$-A concentration difference measurement. It almost exclusively measures cerebral cortex metabolism, since superior sagittal sinus blood flow represents mainly cerebral cortical blood flow $(3,5,6,18,24)$. This assumption is based on anatomical considerations (25-27).

Arteriovenous shunting and consequently arterialization of venous blood can be a problem in organ flux studies, but probably plays no role in our method, as the rat brain lacks arteriovenous shunts $(6,17)$. A major problem in cerebral blood flow and flux measurement resides in the amount of extracerebral contamination. On this subject, contradictory data have been published $(5,6,17,24)$. However, at least three factors seem to preclude extracerebral contamination of the SSS blood in our model. 
First, the central position of the sampling catheter reduces the risk of extracerebral contributions to the SSS flux (5). Small perforating vessels in the diploe are the only extracranial contributories in the vicinity of the sampling catheter, but as intracranial pressure is slightly higher than extracranial, flow is probably directed from inside out in the physiological situation (6).

Second, our sampling rate is approximately forty times slower than that used by Hertz and Bolwig (17). These authors, using ${ }^{24} \mathrm{Na}^{+}$as an indicator of extracerebral contamination, found 18 and $12.5 \%$ extracerebral contamination in artificially ventilated and spontaneously breathing rats, respectively (17). The much slower sampling rate in our spontaneously breathing rats ensures negligible extracerebral contamination.

Finally, the RQ of 1.1 demonstrates that blood was sampled from tissue solely utilizing glucose as a fuel, consistent with substrate utilization in brain and making extracerebral contamination unlikely. From these considerations, we conclude that the samples taken from the confluence catheter represent cerebral cortical venous outflow.

In our experiments SSS blood flow was increased during hypercapnia, which is in agreement with others' findings $(5,6)$ and typical for cerebral blood flow. Also, the intact carbon dioxide-mediated blood flow regulation suggests that our method does not interfere with normal blood flow regulation. Furthermore, these results provide indirect evidence for the reproducibility of this method, since blood flow in all rats responded equally to the $\mathrm{pCO}_{2}$ increase. Additional evidence on this point was derived from repeated blood flow measurements in portacaval shunted rats and rats with acute liver ischemia in a study reported elsewhere (19). These experiments also showed good reproducibility (see 'Results'). Finally, the small standard errors of the mean of the superior sagittal sinus flows reported herein also provide indirect evidence for the reliability of the method.

The cerebral cortical blood flow data obtained with this method are comparable with data in the literature. Total brain blood flow has been reported to be approximately $1 \mathrm{~mL} \mathrm{~min} \mathrm{~m}^{-1}$ in 300 -g rats (17). In other studies blood flow was expressed per gram brain tissue and values ranging from $1 \mathrm{~mL} \cdot \mathrm{g}^{-1} \cdot \mathrm{min}^{-1}(1,3,5,24)$ to $1.64 \mathrm{~mL} \cdot \mathrm{g}^{-1} \cdot \mathrm{min}^{-1}$ (6) were noted. Cortical blood flow was somewhat higher $\left(1.0 \mathrm{~mL} \cdot \mathrm{g}^{-1} \mathrm{~min}^{-1}\right)$ than flow in deeper layers $\left(0.8 \mathrm{~mL} \cdot \mathrm{g}^{-1} \cdot \mathrm{min}^{-1}\right)(1,2)$. Assuming brain weight to be approximately $1.7 \mathrm{~g}(28,29)$ and

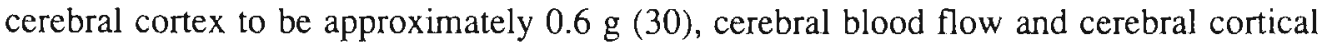
blood flow must be approximately $1.7 \mathrm{~mL} \mathrm{~min}^{-1}$ and $0.6 \mathrm{~mL} \mathrm{~min} \mathrm{~m}^{-1}$, respectively. We found SSS blood flow at basal arterial $\mathrm{pCO}_{2}$ levels to be approximately $540 \mu \mathrm{L} \mathrm{min}^{-1}$, in good agreement with the cerebral cortical blood flow value.

A specific application problem of the indicator dilution principle is that blood flow can be affected by infusion of the indicator $(17,31)$. In our method, this was apparently of little importance since the $30 \mu \mathrm{L} \mathrm{min}{ }^{-1} \mathrm{PAH}$ infusion rate enhanced SSS blood flow by only about $5 \%$. If necessary, this consistent overestimation can be corrected by subtracting the infusion rate from the calculated blood flow. Moreover, we have measured SSS flow at lower PAH infusion rates $\left(18 \mu \mathrm{L} \cdot \mathrm{min}^{-1}\right.$ with a $5 \mathrm{mM}$ PAH solution) in an otherwise similar protocol. In these experiments steady state was obtained after a 20 -minute infusion and yielded similar flows (data not shown).

Another potential problem of flow measurement by the indicator dilution technique is the occurrence of laminar flow in the vessel in which blood flow is measured (31). Laminar flow could interfere with adequate mixing of the indicator with the blood and could thereby lead 
to loss of accuracy, increased variation among animals and sensitivity to small changes of catheter position. Although we have not excluded the presence of strictly laminar flow in the superior sagittal sinus, the good reproducibility and the relatively small standard errors suggest that inadequate mixing does not constitute a major problem in the present method.

Cerebral cortex metabolic rate of $\mathrm{O}_{2}$ consumption was approximately $25 \%$ of the values reported in the literature for whole brain (3). Superior sagittal sinus flux represents $30 \%$ of total brain flux (18), which agrees with our data.

In normal rats, net ammonia uptake and most amino acid fluxes were not significantly different from zero. An undetectable net venous-arterial concentration difference across the normal rat brain agrees with previous reports by others $(32,33)$. In contrast, during hyperammonemia in rats, ammonia flux across the brain showed net uptake $(32,33)$. In a recent study using the method we describe during severe acute hyperammonemia, we also observed a net ammonia uptake and a release of glutamine across the cerebral cortex (19), demonstrating that the model is sensitive enough to measure these differences.

Gjedde et al. (33) reported net whole brain glutamine release in normal as well as hyperammonemic rats. We could not confirm this by cerebral cortex flux measurements for normal rats, but found net cerebral cortex glutamine release during severe hyperammonemia (19). Uptake of branched chain amino acids by the brain has been shown to occur in sheep, dogs and humans (32 and references quoted therein). We found no net BCAA uptake, which might be attributable to regional differences in cerebral metabolism (34). The same might apply to the observed alanine release. However, we only measured fluxes in a limited number of rats and, therefore, firm conclusions do not seem justified.

In conclusion, the method we describe is simple, economical, and enables repeated in vivo measurement of cerebral cortical blood flow and fluxes, which may contribute to a reduction of the amount of animals needed for multiple time point studies.

\section{REFERENCES}

1. Goldman H, Sapirstein LA. Brain blood flow in the conscious and anesthetized rat. Am J Physiol 1973:224:122-126.

2. van Uitert RL, Levy DE. Regional brain blood flow in the conscious gerbil. Stroke 1978;9:67-72.

3. Takei H, Fredericks WR, London ED, Rapoport SI. Cerebral blood flow and oxidative metabolism in conscious Fischer-344 rats of different ages. J Neurochem 1983;40:801-805.

4. van Uiter RL, Sage JI, Levy DE, Duffy TE. Comparison of radio-labeled butanol and iodoantipyrine as cerebral blood flow markers. Brain Res 1981;222:365-372.

5. Gjedde A, Caronna JJ, Hindfelt B, Plum F. Whole-brain blood flow and oxygen metabolism in the rat during nitrous oxide anesthesia. Am J Physiol 1975;229:113-118.

6. Norberg K, Siesjö BK. Quantitative measurement of blood flow and oxygen consumption in the rat brain. Acta Physiol Scand 1974;91:154-164.

7. Miller BM, Cersosimo E, McRae J, Williams PE, Lacy WW, Abumrad NN. Interorgan relationships of alanine and glutamine during fasting in the conscious dog. J Surg Res 1983;35:310-318.

8. Johnson DJ, Muhlbacher F, Wilmore DW. Measurement of hepatic blood flow. J Surg Res 1985;39:470-481.

9. Souba WW, Roughneen PT. Goldwater DL, Williams JC, Rowlands RJ. Postoperative alterations in interorgan glutamine exchange in enterectomized dogs. J Surg Res 1987;42:117-125.

10. Muhlbacher F. Kapadia CJ, Colpoys MF, Smith RJ, Wilmore DW. Effects of glucocorticoids on glutamine metabolism in skeletal muscle. Am J Physiol 1984;247:E75-83.

11. Ardawi MSM, Jamal YS. Glutamine metabolism in skeletal muscle of glucocorticoid-treated rats. Clin Sci 1990;79:139-147. 
12. Urban E, Zingeri AA. A simple method of cannulating the portal vein and obtaining multiple blood samples in the rat. Experientia 1981;37:1036-1037.

13. Welboume TC. Effect of metabolic acidosis on hindquarter glutamine and alanine release. Metabolism 1986;35:614-618.

14. Dejong CHC, Kampman MT, Deutz NEP, Soeters PB. Altered glutamine metabolism in rat portal drained viscera and hindquarter during hyperammonemia. Gastroenterology 1992; 102:936-948.

15. Katz ML, Bergman EN. Simultaneous measurements of hepatic and portal venous blood flow in the sheep and dog. Am J Physiol 1969;216:946-952.

16. Gilbert R. Spirometry and blood gases. In Henry JB, ed. Clinical diagnosis and management by laboratory methods. W.B. Saunders, Philadelphia, 1984.

17. Hertz MM, Bolwig TG. Blood-brain barrier studies in the rat: an indicator dilution technique with tracer sodium as an intemal standard for estimation of extracerebral contamination. Brain Res 1976; 107:333-343.

18. Park TS, van Wylen DGL, Rubio R, Beme RM. Interstitial fluid adenosine and sagittial sinus blood flow during bicucull line seizures in newborn piglets. J Cer Bl Fl Metab 1987;7:633-639.

19. Dejong CHC. Deutz NEP, Soeters PB. Enhanced net ammonia uptake and glutamine release by rat cerebral cortex during acute hepatic encephalopathy. J Hepatol 1990; 10:S6 (abstract).

20. Brun C. A rapid method for the determination of para-aminohippuric acid in kidney function tests. J Lab Clin Med 1951;37:955-958.

21. van Eijk HMH, van der Heijden MAH, van Berlo CLH, Soeters PB. Fully automated liquidchromatographic determination of amino acids. Clin Chem 1988;34:2510-2513.

22. Neeley WE, Philipson J. Automated enzymatic method for determining ammonia in plasma, with 14day reagent stability. Clin Chem 1988;34:1868-1869.

23. Norusis MJ ed. SPSS/PC+ V3.1 BASE MANUAL for the IBM PC/XT/AT and PS/2. SPSS Inc, Chicago, USA, 1989.

24. Norberg K, Siesjö BK. Metabolism of oxygen, glucose, lactate and pyruvate in the rat brain in situ. J Neurochem 1974;22:1127-1129.

25. Green EC. Anatomy of the rat. Hafner Publishing Company, NY, London, 1963.

26. Bardosi A, Ambach G. Constant position of the superficial cerebral veins of the rat: a quantitative analysis. Anat Rec 1985;211:338-341.

27. Schumacher M. Microangiographic study of the normal anatomy of the cerebral venous system in rats. Neuroradiology 1984;26:137-140.

28. Caster WO, Poncelet J, Simon AB, Armstrong WD. Tissue weights of the rat. I. Normal values determined by dissection and chemical methods. Proc Soc Exp Biol Med 1956;91:122-126.

29. Uezu N, Yamamoto S, Rikimaru T, Kishi K, Inoue G. Contributions of individual body tissues to nitrogen excretion in adult rats fed protein deficient diets. J Nutr 1983;113:105-114.

30. Sage JI, van Uitert RL, Duffy TE. Simultaneous measurement of cerebral blood flow and unidirectional movement of substances across the blood-brain barrier: Theory, method, and application to leucine. J Neurochem 1981;36:1731-1738.

31. Granger DN, Bulkley GB. Measurement of blood flow. Applications to the splanchnic circulation. Williams \& Wilkins, Baltimore/London, 1981.

32. Cooper AЛ, Plum F. Biochemistry and physiology of brain ammonia. Physiol Rev 1987;67:440-519.

33. Gjedde A, Lockwood AH, Duffy TE, Plum F. Cerebral blood flow and metabolism in chronically hyperammonemic rats: effect of an acute ammonia challenge. Ann Neurol 1978;3:325-330.

34. Butterworth RF, Girard G, Giguère JF. Regional differences in the capacity for ammonia removal by brain following portacaval anastomosis. J Neurochem 1988;51:486-490. 


\title{
Chapter 6
}

\section{Cerebral cortex during acute liver failure}

\author{
B. Ammonia and amino acid exchange ${ }^{2}$
}

Published as: C.H.C. Dejong. M.T. Kampman, N.E.P. Deutz, P.B. Soeters. Cerebral cortex ammonia and glutamine metabolism during liver insufficiency-induced hyperammonemia in the rat. $J$ Neurochem 1992;59:1071-1079.

\begin{abstract}
Hyperammonemia has been suggested to induce enhanced cerebral cortex ammonia uptake, subsequent glutamine synthesis and accumulation, and finally net glutamine release into the blood stream, but this has never been confirmed in liver insufficiency models. Therefore, cerebral cortex ammonia- and glutamine-related metabolism was studied during liver insufficiency-induced hyperammonemia by measuring plasma flow and venous-arterial concentration differences of ammonia and amino acids across the cerebral cortex (enabling estimation of net metabolite exchange), 1 day after portacaval shunting and 2,4 and $6 \mathrm{~h}$ after hepatic artery ligation (or in controls). The intra-organ effects were investigated by measuring cerebral cortex tissue ammonia and amino acids $6 \mathrm{~h}$ after liver ischemia induction or in controls.

Arterial anmonia and glutamine increased in portacaval shunted rats versus controls, and further increased during liver ischemia. Cerebral cortex net ammonia uptake, observed in portacaval shunted rats, increased progressively during liver ischemia, but net glutamine release was only observed after $6 \mathrm{~h}$ of liver ischemia. Cerebral cortex tissue glutamine, $\boldsymbol{\gamma}$ aminobutyric acid, most other amino acids and ammonia levels were increased during liver ischemia. Glutamate was equally decreased in portacaval shunted and liver ischemia rats.
\end{abstract}

\footnotetext{
2 This chapter is an exact copy of the original publication and consequently does not present all data of the experiments. Additional data are available on request.
} 
The observed net cerebral cortex ammonia uptake, cerebral cortex tissue ammonia and glutamine accumulation and finally glutamine release into the blood, suggest that the rat cerebral cortex initially contributes to net ammonia removal from the blood during liver insufficiency-induced hyperammonemia by augmenting tissue glutamine and ammonia pools, and later by net glutamine release into the blood. The changes in cerebral cortex glutamate and GABA could be related to altered ammonia metabolism.

\section{INTRODUCTION}

Liver disease, whether acute or chronic, is generally accompanied by hyperammonemia, resulting from diminished hepatic urea synthesis capacity and portal-systemic shunting $(1,2)$. In these hyperammonemic states, glutamine synthesis in glutamine synthetase containing organs is the most important alternative pathway for ammonia disposal $(1,3)$.

Muscle has been suggested to be the principle organ synthesizing and releasing glutamine during hyperammonemia $(3,4)$ and the glutamine release by skeletal muscle is generally thought to explain the high plasma glutamine levels usually observed in hyperammonemic states (5). However, recently (6) we could not confirm this hypothesis in rats made acutely hyperammonemic by liver ischemia. The question arose whether muscle actually is an important source of the elevated plasma glutamine during acute hyperammonemia.

Although brain has higher glutamine synthetase activity than muscle (7), the role of the brain in inter-organ ammonia and glutamine exchange has not yet been conclusively established (8). Ammonia has been shown to be taken up by the brain in various hyperammonemic states $(4,8-10)$, but net ammonia removal by the brain has not yet been quantified during acute liver ischemia-induced hyperammonemia. Also, reports on net cerebral glutamine release are sparse and limited to chronic hyperammonemia (5).

Theoretically, net cerebral ammonia uptake could be important in inter-organ nitrogen exchange during hyperammonemia by exerting a systemic hyperammonemia-lowering effect, but this process could have deleterious effects within the brain. Ammonia is an important nitrogenous compound in several reactions in the brain, but at elevated levels it is neurotoxic $(2,10,11)$. Moreover, ammonia has been hypothesized to deplete cerebral glutamate pools during hyperammonemia by enhancing glutamine synthesis $(2,8,12)$ and stimulating subsequent glutamine release into the blood $(5,8,10)$. Because glutamate is an important excitatory neurotransmitter in several regions of the central nervous system, including the cerebral cortex $(8,13,14)$, metabolic ammonia removal might adversely affect neurotransmission. Thus, it appears that during hyperammonemia the exchange of ammonia and glutamine between organs might have serious consequences within organs, particularly in the brain.

Recent observations suggest that during hyperammonemia, alterations in glutamate (14), ammonia, glutamine and glutamine synthetase $(15,16)$ differ between cerebral cortex and the remaining brain. During acute liver ischemia in rats (14), we found that glutamate concentrations were unchanged in whole brain, decreased in cerebral cortex and increased in the remaining brain, stressing the need to study brain regions separately. Therefore, we recently developed an in vivo rat model to study ammonia and amino acid exchange across 
the cerebral cortex (17), as part of more elaborate studies on the inter-organ exchange of ammonia and amino acids during acute liver insufficiency-induced hyperammonemia (6).

The present study specifically addresses the role of the cerebral cortex in net ammonia removal from the blood (as an ammonia-lowering modality) during acute liver ischemiainduced hyperammonemia in the rat. To this purpose ammonia and amino acid exchange was assessed by measuring plasma flows and venous-arterial concentration differences across the cerebral cortex. In addition, the intra-cerebral consequences of cerebral cortex ammonia removal were studied by measuring cortical tissue ammonia and amino acid concentrations.

The present experiments support the hypothesis that acute liver insufficiency-induced hyperammonemia leads to enhanced cerebral cortex ammonia uptake, to ammonia and glutamine accumulation within the cerebral cortex, and finally to glutamine release into the blood.

\section{MATERIALS AND METHODS}

Materials Intramedic ${ }^{R}$ Polyethylene Tubing was obtained from Clay Adams (Parsippany, NJ, U.S.A.). Para-aminohippuric acid (PAH) was from Sigma Chemicals (Sigma A 1422, St. Louis, MO, U.S.A.).

Animals Male SPF Wistar rats (250 \pm 25 g, Winkelmann, Borchen, F.R.G.), housed under standard conditions ( $12 \mathrm{~h}$ light cycle: 8 AM - 8 PM), fed standard lab chow and water ad libitum, were used throughout. Rats were maintained in accordance with the recommendations of the Guide for the Care and Use of Laboratory Animals, as applied in our institute. All surgical procedures were performed by licensed personnel under the auspices of the licensing authority of the Centralized Animal Facilities of our institute.

Experimental groups Before surgery, rats were assigned randomly to one of the following groups:

CON Day 0: laparotomy and manipulations as in PCS rats, but without shunting.

PCS Day 0: portacaval shunting. Day 2: SHAM operation mimicking acute liver ischemia induction, but omitting hepatic artery ligation.

ALI Day 0: portacaval shunting. Day 2: hepatic artery ligation to induce acute liver ischemia.

Surgical procedures Operations and sampling procedures were carried out under ether anesthesia at constant core temperatures. Portacaval shunting was performed by the button technique, as described previously (6). Acute liver ischemia was induced by ligating the hepatic artery in previously portacaval shunted rats.

Catheter implantation (Day 1) (17) (Chapter 6A) All vessels were cannulated with PE 10 tubing $(0.30 \mathrm{~mm} \mathrm{ID}, 0.58 \mathrm{~mm}$ OD). A catheter was inserted via the femoral artery into the abdominal aorta and two catheters were inserted into the superior sagittal sinus, which drains predominantly cerebral cortical venous blood (18). To accomplish this, the skull was exposed through a sagittal incision from the midpoint between the eyes to the interscapular region. With a drill the bone was milled away anterior to the bregma and posterior to the lambdoid suture, exposing $5 \mathrm{~mm}$ of the anterior and $5 \mathrm{~mm}$ of the posterior part of the superior sagittal sinus. Then, the catheters were inserted as described in part A of this chapter. The tip of the anterior catheter was positioned at the bregma, the tip of the posterior 
Table 1. Experimental groups.

\begin{tabular}{|c|c|c|c|c|}
\hline Group & $n$ & day 0 & day 1 & day 2 \\
\hline CON & 5 & SHAM & $\begin{array}{l}\text { catheter implantation } \\
\text { sampling at to }\end{array}$ & \\
\hline PCS $0,2,4,6$ & $32^{\prime \prime}, 5,6,6$ & PCS & $\begin{array}{l}\text { catheter implantation } \\
\text { sampling at to (PCSO) }\end{array}$ & $\begin{array}{l}\text { SHAM at to, sampling } 2,4 \\
\text { or } 6 \mathrm{~h} \text { later. }\end{array}$ \\
\hline ALI 2,4,6 & $11,13,14$ & PCS & $\begin{array}{l}\text { catheter implantation } \\
\text { sampling at to (PCSO) }\end{array}$ & $\begin{array}{l}\text { ALI at to, sampling } 2,4 \\
\text { or } 6 \mathrm{~h} \text { later }\end{array}$ \\
\hline
\end{tabular}

PCS: PortaCaval Shunting, ALI: Acute Liver Ischemia, SHAM: SHAM operation, for details cf. 'Surgical procedures'. " PCSO rats were either subjected to SHAM or ALI operation on day 2; to keep the table clear, all PCSO rats have been grouped in the PCS category.

catheter was positioned in the confluence of sinuses. Both catheters were cemented in place with cyanoacrylate rapid binding adhesive and the posterior catheter was secured additionally with a suture at the occipital notch. The anterior catheter was used for para-aminohippuric acid infusion for flow determinations, the posterior catheter for blood sampling. All catheters were heparinized (50 IU $\mathrm{mL}^{-1}$ heparin in $0.9 \% \mathrm{NaCl}$ ), sealed, coded, and burrowed subcutaneously in the neck after tunneling.

Sampling procedures Rectal temperature was maintained at pre-operative levels: ALI rats at $34 \pm 1^{\circ} \mathrm{C}$, other rats at $37 \pm 1^{\circ} \mathrm{C}(19)$. An isomolaric, pH adjusted, $50 \mathrm{mM}$ PAH solution was infused in the anterior superior sagittal sinus catheter $\left(35 \mu \mathrm{L} \mathrm{min}^{-1}\right.$, Minipuls 2 pump, Gilson France SA, Villiers le Bel, France) allowing for $20 \mathrm{~min}$ equilibration prior to blood sampling.

One $\mathrm{mL}$ of arterial and venous blood were simultaneously collected on ice. Venous blood was sampled semi-automatically at a rate of $50 \mu \mathrm{L} \cdot \mathrm{min}^{-1}$ in heparinized cups (Microvette CB1000, Sarstedt, Nümbrecht, F.R.G.). Arterial samples were collected freely flowing. After sampling a blood transfusion was given into the arterial catheter. Hereafter, all catheters were heparinized, sealed and burrowed subcutaneously in the neck and the rats were placed back in their cages (except at $t=6 \mathrm{~h}$ ). Blood samples were kept on ice during transport to the laboratory for further processing within $10 \mathrm{~min}$.

Tissue sampling In some rats after blood sampling (cf. Tables 1 and 4), the cortex of one hemisphere was collected as described previously (14), freeze clamped in liquid nitrogen and stored at $-70^{\circ} \mathrm{C}(6)$. This procedure is accomplished within $1 \mathrm{~min}$, yields results similar to the freeze blowing technique (20) and warrants reliable tissue ammonia and amino acid determinations, especially for $\gamma$-aminobutyric acid (GABA) (20,21). Apart from the experimental rats, cerebral cortex was sampled in a series of 4 previously unoperated normal rats.

Chronological sequence of the experiments (Table 1) On day 0, portacaval shunting or corresponding SHAM operation was performed. After surgery rats were housed individually. On day 1, catheters were inserted into the superior sagittal sinus and the femoral artery. Blood was sampled in CON and PCS rats (PCSO) and restituted in PCS rats by an blood transfusion (furst transfusion); CON rats were killed after cerebral cortex sampling. On day 2 , in PCS rats at $t=0 \mathrm{~h}$ the hepatic artery was ligated (ALI) or the corresponding SHAM operation (PCS) was performed. Hourly, ALI rats received $1 \mathrm{~mL}$ of $5 \%$ glucose solution i.p. 
(19), temperature was measured and coma grade was estimated using a 6 stage coma scale (14).

Blood was sampled twice in the same rat randomly at either 2 and $6 \mathrm{~h}$, or 4 and $6 \mathrm{~h}$ after liver ischemia induction or its SHAM operation. After sampling at 2 or $4 \mathrm{~h}$ a blood transfusion was given (second transfusion) (Table 1).

Tissue processing For tissue ammonia and amino acid determinations, 5\% (w/v) sulfosalicylic acid extracts were prepared (6). Water content was determined after freeze-drying and calculated as described previously (22).

Biochemical analysis Centrifugations were performed at $4^{\circ} \mathrm{C}$ during 5 min at $8,800 \mathrm{~g}$. All samples were stored

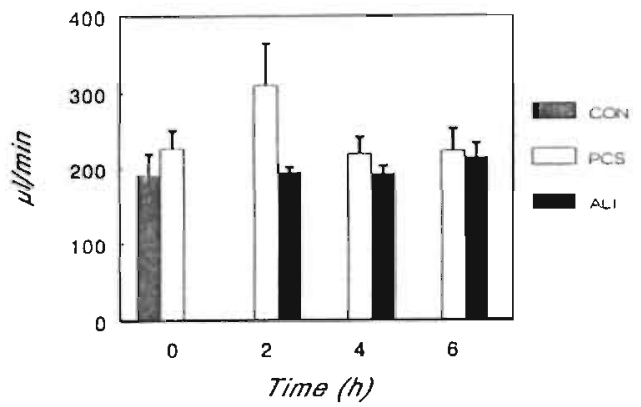
at $-70^{\circ} \mathrm{C}$. Hematocrit was obtained with a microfuge. Ammonia, lactate and glucose were determined by standard enzymatic methods (6), amino acids were determined by an HPLC technique (23). PAH was determined spectrophotometrically as described previously (6). The coefficient of variance for all determinations was well below $4 \%$ (e.g. $2 \%$ for glutamine) $(6,23)$.

Calculations Venous (confluence) concentrations were corrected for the dilution by the PAH infusion. $\alpha$-Amino nitrogen was calculated as the sum of amino acids measured (23) (Table 2). Plasma PAH concentration was calculated as $[\mathrm{PAH}]_{\text {plasma }}=[\mathrm{PAH}]_{\mathrm{blood}}$. $100 /(100-\mathrm{Ht})$. Fluxes (nmol $\mathrm{min}^{-1}$ ) were calculated as mean flow per group times individual $\mathrm{V}$-A difference (mean group flow was used, because flow data were not available in all individual rats of one group). A positive figure means net efflux, negative values reflect net uptake. Plasma flows were calculated as $(6,17)$ :

$$
F_{S S S}=\frac{1}{[P A H]_{C}-[P A H]_{A}}
$$

In this formula, $F_{\text {sss }}$ is superior sagittal sinus plasma flow $\left(\mathrm{mL} \cdot \mathrm{min}^{-1}\right), I$ is the infused PAH $\left(\mu \mathrm{mol} \mathrm{min}^{-1}\right)$, and $[\mathrm{PAH}]_{\mathrm{C}(\mathrm{A})}$ is the confluence $(\mathrm{C})$ and arterial (A) plasma PAH concentration $(\mu \mathrm{M})$, respectively.

Statistical analysis (24). Data presented are means $\pm 1 S E M, P<0.05$ was considered significant. Analysis of variance was used to test for group effects (ANOVA) and time effects within groups (ONEWAY). The Mann-Whitney $U$ non-parametric test was used for differences between groups at specific time points. Wilcoxon's nonparametric test was used for differences from zero. Significances are indicated in Tables 2, 3, \& 4. 
Table 2. Arterial plasma concentrations in control (CON), portacaval shunted (PCS) and acute liver ischemia rats (ALI).

\begin{tabular}{lrrrrrrrr} 
& \multicolumn{1}{c}{ CON } & \multicolumn{1}{c}{ PCSO } & \multicolumn{1}{c}{ PCS2 } & \multicolumn{1}{c}{ PCS4 } & \multicolumn{1}{c}{ PCS6 } & \multicolumn{1}{c}{ ALI2 } & \multicolumn{1}{c}{ AL14 } & \multicolumn{1}{c}{ ALl6 } \\
\hline Amm & $121 \pm 38$ & $261 \pm 19^{\prime}$ & $113 \pm 11$ & $149 \pm 28$ & $117 \pm 19$ & $530 \pm 56^{\times}$ & $989 \pm 39^{3}$ & $1472 \pm 35^{\circ}$ \\
GLU & $91 \pm 14$ & $90 \pm 7$ & $84 \pm 21$ & $85 \pm 21$ & $54 \pm 9$ & $182 \pm 31$ & $218 \pm 20^{3}$ & $333 \pm 39^{\circ}$ \\
GLN & $543 \pm 9$ & $861 \pm 82^{m}$ & $663 \pm 35$ & $786 \pm 60$ & $664 \pm 62$ & $2153 \pm 179^{\circ}$ & $3124 \pm 194^{3}$ & $4585 \pm 324^{\circ}$ \\
ALA & $399 \pm 49$ & $641 \pm 76^{k}$ & $659 \pm 88$ & $673 \pm 111$ & $519 \pm 96$ & $2374 \pm 272^{\gamma}$ & $2654 \pm 151^{3}$ & $3537 \pm 184^{\circ}$ \\
TYA & $56 \pm 5$ & $95 \pm 12^{\prime}$ & $109 \pm 12$ & $136 \pm 25$ & $128 \pm 27$ & $334 \pm 67^{\times}$ & $480 \pm 24^{3}$ & $654 \pm 34^{\circ}$ \\
PHE & $58 \pm 5$ & $103 \pm 9^{m}$ & $131 \pm 10$ & $137 \pm 15$ & $147 \pm 18$ & $307 \pm 34^{\times}$ & $501 \pm 27^{3}$ & $665 \pm 25^{\circ}$ \\
TRP & $58 \pm 4$ & $75 \pm 3^{\prime}$ & $84 \pm 7$ & $77 \pm 4$ & $63 \pm 3$ & $97 \pm 13$ & $126 \pm 11^{\prime}$ & $123 \pm 13^{\circ}$ \\
BCAA & $235 \pm 19$ & $294 \pm 26$ & $470 \pm 42$ & $501 \pm 46$ & $587 \pm 56$ & $704 \pm 113$ & $951 \pm 53^{2}$ & $1153 \pm 60^{\circ}$
\end{tabular}

Values are expressed as means $\pm 1 S E M$ in $\mu \mathrm{mol} \cdot \mathrm{L}^{-4}$. Symbols: ANOVA for differences between ALI and PCS; $t=0$ to $t=6 \mathrm{~h}$ :

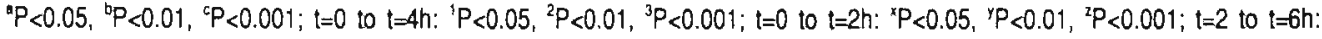
${ }^{P} P<0.05,{ }^{9}<<0.01,{ }^{\prime} P<0.001$. Mann-Whitney $U$ for differences between $C O N$ and $P C S O$; ${ }^{x}<<0.05,{ }^{2} P<0.01,{ }^{m} P<0.001$. The changes in arterial concentrations in this study were comparable to those previously observed (6). Therefore, only some amino acids are shown.

Table 3. Cerebral cortex fluxes in control (CON), portacaval shunted (PCS) and acute liver ischemia rats (ALI).

\begin{tabular}{lccccccccccc} 
& CON & PCSO & \multicolumn{1}{c}{ PCS2 } & \multicolumn{1}{c}{ PCS4 } & \multicolumn{1}{c}{ PCS6 } & \multicolumn{1}{c}{ ALI2 } & \multicolumn{1}{c}{ ALI4 } & \multicolumn{2}{c}{ ALI6 } \\
\hline $\mathrm{FE}_{\text {Anm }}$ & $10 \pm 10$ & $10 \pm 3$ & $21 \pm 6$ & $5 \pm 2$ & $7 \pm 11$ & $15 \pm$ & 7 & $13 \pm$ & 4 & $7 \pm 4$ \\
GlC & $-60 \pm 100$ & $-86 \pm 58$ & $-132 \pm 84$ & $-103 \pm 60$ & $-157 \pm$ & $50^{+}$ & $-54 \pm 30$ & $-141 \pm$ & $2 T^{+}$ & $-120 \pm 30$ \\
Amm & $-3.9 \pm 3.3$ & $-8.1 \pm 2.4^{+}$ & $-12.4 \pm 5.3^{+}$ & $-2.1 \pm 1.4$ & $-3.0 \pm 2.7$ & $-16.6 \pm 8.2^{+}$ & $-24.4 \pm 7 . T^{+}$ & $-26.7 \pm 12.6$ \\
GLU & $-0.4 \pm 1.1$ & $0.2 \pm 0.5$ & $-1.5 \pm 2.0$ & $-3.9 \pm 4.9$ & $0.1 \pm 0.9$ & $1.3 \pm 1.0$ & $-3.3 \pm 4.0$ & $-5.4 \pm 5.0$ \\
GLN & $-2.7 \pm 5.9$ & $0.8 \pm 3.5$ & $4.0 \pm 6.6$ & $-9.1 \pm 7.9$ & $-3.8 \pm 8.1$ & $17.1 \pm 13.6$ & $17.6 \pm 17.2$ & $54.2 \pm 17.1$ \\
TYR & $-0.0 \pm 0.3$ & $0.6 \pm 0.2^{+}$ & $-0.4 \pm 1.3$ & $-0.6 \pm 1.3$ & $0.9 \pm 1.4$ & $2.4 \pm 1.6$ & $-2.0 \pm 2.7$ & $-0.2 \pm 3.2$ \\
TRP & $-1.2 \pm 0.4^{+}$ & $-0.5 \pm 0.3$ & $-1.5 \pm 1.4$ & $-0.2 \pm 0.5$ & $-0.2 \pm 0.6$ & $1.4 \pm 0.6$ & $-0.8 \pm 0.7$ & $-0.2 \pm 1.0$ \\
BCAA & $-1.0 \pm 1.9$ & $-1.6 \pm 1.5$ & $-6.1 \pm 8.2$ & $-5.6 \pm 3.6$ & $0.0 \pm 7.0$ & $3.6 \pm 3.5$ & $-7.9 \pm 5.8$ & $-2.7 \pm 6.3$ \\
\hline
\end{tabular}

Values are expressed as means \pm 1 SEM in nmol min ${ }^{-1}$. Of the amino acids measured a selection is shown. No changes were observed in the other amino acids measured. $\mathrm{FE}_{\mathrm{Anm}}$ : Fractional ammonia extraction; Glc: glucose. For significance symbols, see Table 2, except difference from zero (tested by the Wikoxon test): " ${ }^{-}$significantly different from 0 .

\section{RESULTS}

Hepatic encephalopathy stages As expected, all rats with liver ischemia developed hepatic encephalopathy, which progressed to severe ataxia and loss of spontaneous righting reflex at $\mathrm{t}=6 \mathrm{~h}$, following a course similar to that described previously $(6,19,20,25)$. However, none of the rats with acute liver ischemia in the present study became comatose. In all other rats we did not observe behavioral changes.

Arterial concentrations (Table 2) and plasma flows (Figure 1) Superior sagittal sinus

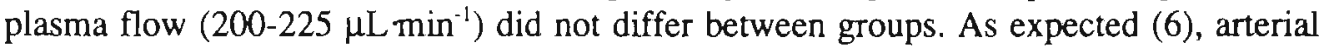
ammonia (Figure 2) and glutamine (Figure 3) were elevated in PCS rats and increased progressively to approximately 1500 and $4600 \mu \mathrm{M}$, respectively, after liver ischemia 
Table 4: Cerebral cortex tissue concentrations in normal (NORM), in control (CON), portacaval shunted (PCS) and acure liver ischemia rats $(A L I)$.

\begin{tabular}{|c|c|c|c|c|}
\hline & NOAM (4) & $\operatorname{CON}(4)$ & PCS6 (6) & ALI6 (16) \\
\hline AMM & $287 \pm 20$ & $331 \pm 170$ & $702 \pm 162^{\prime}$ & $2527 \pm 579$ \\
\hline GLU & $11993 \pm 942$ & $10610 \pm 557$ & $9141 \pm 707^{1}$ & $9266 \pm 305$ \\
\hline ASN & $95 \pm \quad 7$ & $81 \pm$ & $89 \pm$ & $112 \pm$ \\
\hline GLN & $5581 \pm 267$ & $6424 \pm 209$ & $10334 \pm 927^{16}$ & $17019 \pm 1129^{2 c y}$ \\
\hline ALA & $670 \pm 24$ & $862 \pm 59^{\prime}$ & $699 \pm 141$ & $1256 \pm \quad 80^{20 y}$ \\
\hline TAU & $6051 \pm 261$ & $6060 \pm 500$ & $4545 \pm 351^{\prime}$ & $5170 \pm 127^{1 x}$ \\
\hline GABA & $2391 \pm 163$ & $2429 \pm 238$ & $2115 \pm 258$ & $3110 \pm 224^{2 y}$ \\
\hline ARG & $77 \pm$ & $34 \pm \quad 23$ & $0 \pm$ & $32 \pm \quad 23^{2}$ \\
\hline TYR & $80 \pm$ & $93 \pm$ & $163 \pm 21^{\circ}$ & $395 \pm 14^{2 c x}$ \\
\hline VAL & $104 \pm$ & $132 \pm$ & $191 \pm 21^{1}$ & $198 \pm$ \\
\hline ILE & $44 \pm$ & $50 \pm$ & $84 \pm \quad 9^{12}$ & $91 \pm$ \\
\hline PHE & $50 \pm$ & $80 \pm$ & $157 \pm \quad 14^{12}$ & $356 \pm \quad 15^{2 c 2}$ \\
\hline TRP & $16 \pm$ & $22 \pm$ & $31 \pm$ & $51 \pm$ \\
\hline LEU & $90 \pm \quad 11$ & $103 \pm \quad 11$ & $162 \pm \quad 17^{12}$ & $198 \pm$ \\
\hline BCAA & $238 \pm 17$ & $284 \pm 11^{1}$ & $437 \pm \quad 46^{1}$ & $487 \pm \quad 16^{2 c}$ \\
\hline$\alpha \mathrm{AN}$ & $30088 \pm 1609$ & $30047 \pm 1210$ & $30669 \pm 2457$ & $41933 \pm 1192$ \\
\hline
\end{tabular}

$\alpha$ AN: $\alpha$-Amino Nitrogen. Number of rats in parentheses. Values are expressed as means \pm 1 SEM in $\mu$ mol $\mathrm{kg}^{-1}$ wet weight. Significance symbols, Mann-Whitney $U$ for differences between groups: versus NORM ' $P<0.05,{ }^{2} P<0.01$; versus $C O N{ }^{2} P<0.05$, ${ }^{\circ} P<0.01,{ }^{C} P<0.001$; versus $P C S{ }^{x} P<0.05,{ }^{\gamma} P<0.01,{ }^{7} P<0.001$. Not shown: \% $H_{2} O, \alpha A A A, C I T, \alpha A B A, O R N, M E T, S E R, G L Y$, THA, HIS and LYS (complete list of amino acids available on demand).

induction. The arterial hyper-aminoacidemia after acute liver ischemia induction was comparable to previous data in the same model, as was the decrease in arterial arginine levels (6). During liver ischemia arterial glucose levels were decreased but within the normal range (3-10 mM), whereas lactate levels increased compared to PCS controls.

Cerebral cortex fluxes (Table 3, Figures 2 and 3) In most groups, ammonia was taken up by the cerebral cortex and this ammonia uptake increased progressively after liver ischemia induction (Figure 2). Cerebral cortex glutamine flux (Figure 3) was only significantly different from zero in ALI rats at $6 \mathrm{~h}$ after liver ischemia induction. No cortical glutamine release was observed in PCS rats. Net cerebral cortex glutamate as well as BCAA flux were not significantly different from zero in any group. The cerebral cortex fluxes of most of the remaining amino acids as well as the $\alpha$-Amino Nitrogen flux were not significantly different from zero at any time point (data not shown). Thus, of the nitrogen substances measured, ammonia uptake was not balanced by efflux of other metabolites, pointing to net nitrogen retention.

Cerebral cortex glucose uptake was only observed in some groups and did not differ between groups. Lactate fluxes (not shown) did not differ from zero in any group.

Cerebral cortex tissue concentrations (Table 4) Tissue concentrations were measured at the end of the experiment, as indicated in Table 4. Tissue ammonia as well as glutamine levels were elevated in ALI compared to PCS, CON and NORM rats, in agreement with literature data $(8,22,26)$. Tissue levels of glutamate were comparably decreased in PCS and ALI rats compared to CON and NORM. Cortex GABA concentrations were increased in 
ALI rats in comparison to the identical levels in NORM, CON and PCS rats. Virtually all other amino acids were elevated in ALI rats compared to all other groups. Tissue water content did not change (not shown).

\section{DISCUSSION}

In the present study we investigated the role of the cerebral cortex in inter-organ ammonia and glutamine exchange, as well as the potential effects of this metabolite exchange across the cerebral cortex on cerebral cortical metabolism and function during acute liver ischemiainduced hyperammonemia. Acute liver ischemia induction is a well-known reproducible rat model of acute severe hyperammonemia, liver failure and hepatic encephalopathy $(6,14,19,20,25,27,28)$.

Ammonia is an important participant in several reactions in the brain, including the glutamine synthetase (GS; EC 6.3.1.2) reaction, primarily localized in astrocytes (29). Glutamine formed in astrocytes is made available to neurons, providing substrate for neurotransmitter glutamate and GABA. Neuronally released glutamate and GABA, not recaptured by the neuron, can be taken up and recycled to glutamine by the astrocyte: the 'glutamine cycle' $(30,31)$. Despite its physiological importance in these and related reactions, ammonia is directly neurotoxic at elevated levels $(2,10,11)$. For this reason, the astrocytic GS reaction, catalyzing the synthesis of the non-toxic amino acid glutamine from glutamate and ammonia, has been viewed as an ammonia detoxification pathway within the brain (31), but also in interorgan ammonia transport (3).

Unidirectional whole brain ammonia uptake has been confirmed by tracer studies (for review, see (8)), but only one report has been published on net ammonia uptake by the rat brain. In this study net ammonia uptake by the brain in 8 weeks portacaval shunted rats (5) was observed, but not in 4 weeks shunted rats. It was suggested that net venous-arterial concentration differences across the brain are small and difficult to measure (5). In our experiments, net ammonia uptake by the cerebral cortex was found at $48 \mathrm{~h}$ in rats with a portacaval shunt and in rats with acute liver ischemia. This provides new evidence for a role of the brain (or brain regions) in net ammonia removal from the circulation during liver disease. Ammonia uptake progressively increased during acute liver ischemia. The cerebral cortex ammonia uptake could account for a maximum of $3 \%$ of the total intestinal ammonia generation in the same model (6). Since we have observed that other organs like the kidney (data not given) also produce ammonia during acute liver ischemia, we conclude that 
cerebral ammonia removal is insufficient to substantially counter-balance ammonia production in the organism.

The fractional ammonia extraction remained unchanged throughout a wide range of ammonia concentrations, suggesting that cortical ammonia is mainly blood-derived and that uptake is not limited at high ammonia levels. The elevated tissue ammonia levels in portacaval shunted rats and the more pronouncedly elevated levels in acute liver ischemia rats demonstrate that cerebral cortex ammonia removal mechanisms are insufficient to counteract the continuous net ammonia uptake. In addition, they suggest that ammonia removal by the hyperammonemic rat cerebral cortex is at least partly mere ammonia trapping.

In brain, ammonia is rapidly incorporated in glutamine by the glutamine synthetase reaction $(3,12,32)$. Ongoing glutamine synthesis during hyperammonemia has been suggested to explain increased brain glutamine levels in humans and experimental animals $(1,10,14)$. Elevated cerebral cortex tissue glutamine levels were also found in portacaval shunted and acute liver ischemia rats in the present study. Whether elevated brain glutamine levels always lead to net glutamine efflux from the brain has been a matter of considerable debate (reviewed in (8)). The present data show net cerebral cortex glutamine efflux 6 hours after liver ischemia induction, when tissue glutamine levels are strongly elevated. This suggests that the rat cerebral cortex contributes to ammonia detoxification at this stage not only by ammonia trapping and augmenting the tissue glutamine pools, but by releasing glutamine which could transport nitrogen to the kidney for excretion. Glutamine efflux at 2 and 4 hours after liver ischemia induction did not reach statistical significance. We previously demonstrated in ${ }^{1} \mathrm{H}$ NMR spectroscopic and biochemical studies, using the same model, that cortical glutamine levels are already elevated after $2 \mathrm{~h}$ of liver ischemia $(14,33)$. Either these small amounts of glutamine released by the cerebral cortex escape detection in our flux measurements or some threshold value exists above which net release of glutamine into the blood occurs. Alternatively, increased blood brain barrier permeability, as recently observed in a rabbit liver failure model during an identical degree of encephalopathy (severe ataxia, no coma; (34)), could be an explanation.

The glutamine efflux after $6 \mathrm{~h}$ of liver ischemia drains the cerebral cortex glutamate pool (8). Therefore, glutamate fluxes could be of special interest, but no cerebral cortex glutamate uptake was observed in any group, probably due to the impermeability of the blood brain barrier for glutamate $(8,35)$. Alternatively, branched chain amino acid uptake and subsequent transamination could replenish the glutamate pool (36-38), but our data do not support 
this theory either, in agreement with the in vitro observation that BCAA metabolism is inhibited during acutely elevated ammonia levels (38).

The changes in ammonia and glutamine exchange across the cerebral cortex during acute liver ischemia-induced hyperammonemia were accompanied by prominent changes in the total cerebral cortex tissue pools of some transmitter amino acids. It is generally agreed that total tissue amino acid levels do not give adequate insight in alterations in neurotransmission. They do not take into account the astrocytic versus neuronal compartment, nor do they permit definitive conclusions on metabolic versus releasable neurotransmitter pools, the latter probably being quite small $(13,30,39,40)$. Nonetheless, some interesting inferences can be drawn from our data.

Cerebral cortex glutamate concentrations decreased during liver ischemia and in portacaval shunted rats to approximately $75 \%$ of normal values, which is in accordance with literature (14,41-44). Astrocytic glutamine synthesis and resulting glutamate consumption cannot be the only explanation of this glutamate decrease, since the astrocytic compartment contains only $7 \%$ of total brain glutamate (12). Hence, even if the astrocytic glutamate concentration became zero, total cortex glutamate would decrease to only $93 \%$. Therefore, glutamate must be decreased in the neuron too. Because neuronal GS activity is low (8), glutamine synthesis in the neuron is not a likely explanation for this glutamate decrease. Ammonia concentrations above $1 \mathrm{mM}$ inhibit the neuronal glutaminase (EC 3.5.1.2) reaction (45-48), which might play a role in the proposed neuronal (and total cortex) glutamate decrease. Similarly, glutaminase inhibition could contribute to the elevated cerebral cortex glutamine levels in our study.

Another striking observation in the present study was the increase in cerebral cortex GABA concentrations in liver ischemia rats, in agreement with literature data on whole brain (20). Increased blood-derived brain uptake of gut-generated $\gamma$-aminobutyric acid not extracted by the liver during severe liver failure has been suggested to contribute to elevated brain GABA levels, but conflicting results have been reported concerning this issue (34,4952). Because brain GABA levels are 1000 -fold higher than plasma GABA concentrations, enhanced GABA uptake during hepatic encephalopathy should be an active process, but at the moment convincing evidence supporting this concept is lacking.

The cerebral cortex GABA rise during acute liver ischemia might be related to the elevated glutamine levels, because a substantial portion of the glutamine pool has been suggested to be linked to GABA synthesis $(13,31,39)$. Support for this concept is found in the simultaneously decreased GABA and glutamine levels in certain brain regions in rats treated with methionine sulfoximine (GS inhibitor) in our lab (unpublished observations) as well as in the results of others (39). The origin of such a glutamine-related GABA increase cannot be deduced from our experiments, because, like glutamate, GABA is compartmentalized (30). Within the neuronal compartment the two forms of GABA synthesizing glutamate decarboxylase (GAD, EC 4.1.1.15) enzyme are differentially distributed and regulated $(53,54)$, the larger of these probably by selective feedback control by GABA (54). Alternatively, hyperammonemia-induced TCA cycle inhibition (8) could cause increased flux through the GABA shunt pathway, but its relation to increased GABA concentrations remains unclear.

Despite an enormous amount of research (for review see $(8,52,11)$ ) the mechanisms responsible for the pathogenesis of hepatic encephalopathy remain unresolved. It is believed, however, that ammonia metabolism and GABAergic neurotransmission are the 
two major systems involved (52). Although it was not the objective of this study to investigate specifically the pathogenesis of hyperammonemia-associated encephalopathy, the data presented are compatible with the current neurotransmitter imbalance hypothesis of hepatic encephalopathy $(8,14,16,28,44,52)$.

In conclusion, the present study during acute liver insufficiency-induced hyperammonemia, demonstrates that the cerebral cortex of the rat contributes to net ammonia detoxification in this situation initially by augmenting tissue ammonia and glutamine pools, and later by net glutamine release into the blood. This glutamine release could provide the kidney with glutamine for ammonia excretion. The observed cortical glutamine release during late phases of acute liver ischemia-induced hyperammonemia does not seem to be an important factor in explaining the arterial glutamine increase. However, cerebral cortex ammonia uptake, glutamine accumulation by enhanced synthesis and/or breakdown inhibition, and subsequent glutamine release into the blood, could be related to the decreased cortical glutamate and increased GABA levels during acute liver ischemia. These changes might adversely affect cerebral cortex metabolism and function.

\section{REFERENCES}

I. Zieve L. Pathogenesis of hepatic encephalopathy. Metab Brain Dis 1987;2:147-165

2. Meijer AJ, Lamers WH, Chamuleau RAFM. Nitrogen metabolism and ornithine cycle function. Physiol Rev 1990;70:701-748.

3. Lockwood AH, McDonald JM, Reiman RE, Gelbard AS, Laughlin JS, Duffy TE, Plum F. The dynamics of ammonia metabolism in man Effects of liver disease and hyperammonemia. J Clin Invest 1979;63:449-460.

4. Webster LT, Gabuzda GJ. Ammonium uptake by the extremities and brain in hepatic coma. J Clin Invest 1958;37:414-424.

5. Gjedde A, Lockwood AH, Duffy TE, Plum F. Cerebral blood flow and metabolism in chronically hyperammonemic rats: Effect of an acute ammonia challenge. Ann Neurol 1978;3:325-330.

6. Dejong CHC, Kampman MT, Deutz NEP, Soeters PB. Altered glutamine metabolism in rat portal drained viscera and hindquarter during hyperammonemia. Gastroenterology 1992;102:936-948.

7. Lund P. A radiochemical assay for glutamine synthetase, and activity of the enzyme in rat tissues. Bioch J 1970;118:35-39.

8. Cooper AJL, Plum F. Biochemistry and physiology of brain ammonia. Physiol Rev 1987;67:440-519.

9. Rosado A, Flores G, Mora J, Soberon G. Distribution of an ammonia load in the normal rat. Am J Physiol 1962;203:37-42.

10. Duffy TE, Plum F. Hepatic Encephalopathy, in: The liver: Biology and pathobiology (Arias I, Popper H, Schachter D, Shafritz DA, eds)., pp 693-715. Raven Press, New York, 1982.

11. Butterworth RF. Pathophysiology of hepatic encephalopathy; the ammonia hypothesis revisited. In: Progress in Hepatic Encephalopathy and Metabolic Nitrogen Exchange (Bengtson F, Almdal T, Jeppsson B. Vilstrup H, eds)., Part 1, pp. 9-24. CRC Press, Boca Raton, 1991.

12. Cooper AJL, Mora SN, Cruz NF, Gelbard AS. Cerebral ammonia metabolism in hyperammonemic rats. J Neurochem 1985;44:1716-1723.

13. Fonnum F. Glutamate: a neurotransmitter in the mammalian brain. J Neurochem 1984;42,1-11.

14. Bosman DK, Deutz NEP, de Graaf AA, van der Hulst RWN, van Eijk HMH, Bovée WMMJ, Maas MAW, Jöming GGA, Chamuleau RAFM. Changes in brain metabolism during hyperammonemia and acute liver failure: Results of a comparative 'H-NMR spectroscopy and biochemical investigation. Hepatology 1990;12:281-290.

15. Butterworth RF, Girard G, Giguère JF. Regional differences in the capacity for ammonia removal by brain following portacaval anastomosis. J Neurochem 1988;51:486-490.

16. Giguère JF, Besnard AM, Foumier H, Bergeron M, Girard G, Butterworth RF. Evidence for a functional imbalance between central GABAergic and glutamatergic neurotransmission in portal- 
systemic encephalopathy, in: Advances in ammonia metabolism and hepatic encephalopathy (Soeters PB, Wilson JHP, Meijer AJ, Holm E, eds).. pp. 287-293. Excerpta Medica, Amsterdam, 1988.

17. Dejong CHC, Deutz NEP, Soeters PB. A simple method for in vivo cerebral cortex flux measurement in rats. Lab Anim Sci 1992;42:280-285.

18. Schumacher M. Microangiographic study of the normal anatomy of the cerebral venous system in rats. Neuroradiology 1984;26:137-140.

19. Deutz NEP, Chamuleau RAFM, de Graaf AA, Bovée WMMJ, de Beer R. In vivo ${ }^{31} P$ NMR spectroscopy of the rat cerebral cortex during acute hepatic encephalopathy. NMR Biomed 1988;1:101-106.

20. Mans AM, Saunders SJ, Kirsch RE, Biebuick JF. Correlation of plasma and brain amino acid and putative neurotransmitter alterations during acute hepatic coma in the rat. J Neurochem 1979;32:285292.

21. Perry TL, Hansen S, Gandham SS. Postmontem changes of amino compounds in human and rat brain. J Neurochem 1981;36:406-412.

22. Ganz R, Swain M, Traber P, DalCanto M, Butterworth RF, Blei AT. Ammonia induced swelling of rat cerebral cortex slices: implications for the pathogenesis of brain edema in acute hepatic failure. Metab Brain Dis 1989;4:213-223.

23. van Eijk HMH, van der Heijden MAH, van Berlo CLH, Soeters PB. Fully automated liquidchromatographic determination of amino acids. Clin Chem 1988;34:2510-2513.

24. Norusis MJ. SPSS/PC+ V3I BASE MANUAL for the IBM PC/XT/AT and PS/2 SPSS Inc,. Chicago, USA, 1989.

25. Butterworth RF, Swain M, Bergeron M, Blei AT. Cerebrospinal fluid amino acid changes in relation to neurological status in acute liver failure: studies in freely moving rats with in-dwelling cisterna magna catheters. Hepatology 1990; 12:857 (abstract).

26. Lin S, Raabe W. Ammonia intoxication: effects on cerebral cortex and spinal cord. J Neurochem 1985;44:1252-1258.

27. Chamuleau RAFM, Deutz NEP, de Haan JG, van Gool J. Correlation between electroencephalographic and biochemical indices in acute hepatic encephalopathy in rats. J Hepatol 1987;4:299-306.

28. Bosman DK, van den Buijs CACG, de Haan JG, Maas MAW, Chamuleau RAFM. The effects of benzodiazepine-receptor antagonists and partial inverse agonists on acute hepatic encephalopathy in the rat. Gastroenterology 1991:101:772-781.

29. Martinez-Hemandez A, Bell KP, Norenberg MD. Glutamine synthetase: glial localization in brain. Science 1977; 195:1356-1358.

30. Brainard JR, Kyner E, Rosenberg GA. ${ }^{13} \mathrm{C}$ nuclear magnetic resonance evidence for $\gamma$-aminobutyric acid formation via pyruvate carboxylase in rat brain: a metabolic basis for compartmentation. J Neurochem 1989;53:1285-1292.

31. Waniewski RA. Physiological levels of ammonia regulate glutamine synthesis from extracellular glutamate in astrocyte cultures. J Neurochem 1992;58:167-174.

32. Cooper AJL, McDonald JM, Gelbard AS, Gledhill RF, Duffy TE. The metabolic fate of ${ }^{13} \mathrm{~N}$-labeled ammonia in rat brain. J Biol Chem 1979;254:4982-4992.

33. de Graaf AA, Deutz NEP, Bosman DK. Chamuleau RAFM, de Haan JG, Bovée WMM. The use of in vivo proton NMR to study the effects of hyperammonemia in the rat cerebral cortex. NMR Biomed 1991;4:31-37.

34. Basset ML, Mullen KD, Scholz B, Fenstermacher JD, Jones EA. Increased brain uptake of $\gamma$ aminobutyric acid in a rabbit model of hepatic encephalopathy. Gastroenterology 1990;98:747-757.

35. Oldendorf WH, Szabo J. Amino acid assignment to one of three blood-brain amino acid carriers. Am J Physiol 1976;230:94-98.

36. James JH, Herlin PM. Edwards L, Nachbauer CA, Fischer JE. Effect of infusing the branched chain amino acids on concentrations of amino acids in plasma and on brain catecholamines after total hepatectomy in the rat. Life Sciences 1982;30:1361-1368.

37. Watanabe A, Shiota T, Takei N, Fujiwara M, Nagashima H. Ammonia detoxification by accelerated oxidation of branched chain amino acids in brains of acute hepatic failure rats. Biochem Med Metab Biol 1986;35:367-375.

38. Murthy CRK, Hertz L. Comparison between acute and chronic effects of ammonia on branched-chain amino acid oxidation and incorporation into protein in primary cultures of astrocytes and of neurons. $J$ Neurosci Res 1987;17:271-276. 
39. Fonnum F, Paulsen RP. Comparison of transmitter amino acid levels in rat globus pallidus and neostriatum during hypoglycemia or after treatment with methionine sulfoximine or $\gamma$-vinyl $\gamma$ aminobutyric acid. J Neurochem 1990;54:1253-1257.

40. Maddison JE, Watson WEJ, Dodd PR, Johnston GAR. Alterations in cortical $\left[{ }^{3} \mathrm{H}\right]-\mathrm{kainate}$ and $\alpha-\left[{ }^{3} \mathrm{H}\right]-$ amino-3-hydroxy-5-methyl-4-isoxazolepropionic acid binding in a spontaneous canine model of chronic hepatic encephalopathy. J Neurochem 1991:56:1881-1888.

41. Mattson WJ, Iob V, Sloan M, Coon WW, Turcotte JG, Child CG. Alterations of individual free amino acids in brain during acute hepatic coma. SGO 1970;130:263-266.

42. Hindfelt B, Plum F, Duffy TE. Effect of acute ammonia intoxication on cerebral metabolism in rats with portacaval shunts. J Clin Invest 1977;59:386-396.

43. Albrecht J, Hilgier W. Arginine in thioacetamide-induced hepatogenic encephalopathy in rats: activation of enzymes of arginine metabolism to glutamate. Acta Neurol Scand 1986;73:498-501.

44. Bosman DK, Chamuleau RAFM, Bovée WMMJ, van Dijk JE, Deutz NEP. Amino acid release from cerebral cortex in experimental acute liver failure, studied by in vivo cerebral cortex microdialysis. $J$ Neurochem 1992;59:591-599.

45. Bradford HF, Ward HK. Glutamine as a metabolic substrate for isolated nerve-endings, inhibition by ammonia ions. Bioch Soc Trans 1975;3:1223-1226.

46. Matheson DF, van den Berg CJ. Ammonia and brain glutamine: inhibition of glutamine degradation by ammonia. Bioch Soc Trans 1975;3:525-528.

47. Benjamin AM. Control of glutaminase activity in rat brain cortex in vitro, influence of glutamate, phosphate, ammonium, calcium and hydrogen ions. Brain Res 1981;208:363-377.

48. Kvamme E, Lenda $\mathrm{K}$. Regulation of glutaminase by exogenous glutamate, ammonia and 2oxoglutarate in synaptosomal enriched preparation from rat brain. Neurochem Res 1982; 7:667-678.

49. van Berlo CLH, de Jonge HR, van den Bogaard AEJM, van Eijk HMH, Janssen MA, Soeters PB. $\gamma$ Aminobutyric acid production in small and large intestine of normal and germ-free Wistar rats Influence of food intake and intestinal flora. Gastroenterology 1987;93:472-479.

50. Maddison JE, Dodd PR, Morrison M, Johnston GAR, Farrell GC. Plasma GABA, GABA-like activity and the brain GABA-bezodiazepine receptor complex in rats with chronic hepatic encephalopathy. Hepatology 1987;7:621-628.

51. Roy S, Pomier-Layrargues G, Butterworth RF, Huet P-M. Hepatic encephalopathy in cirrhotic and portacaval shunted dogs: lack of changes in brain GABA uptake, brain GABA levels, brain glutamic acid decarboxylase activity and brain postsynaptic GABA receptors. Hepatology 1988;8:845-849.

52. Basile AS, Jones EA, Skolnick P. The pathogenesis and treatment of hepatic encephalopathy: evidence for the involvement of benzodiazepine receptor ligands. Pharmacol Rev 1991;43:27-71.

53. Kaufman DL, Houser CR, Tobin AJ. Two forms of the $\gamma$-aminobutyric acid synthetic enzyme glutamate decarboxylase have distinct intraneuronal distributions and cofactor interactions. J Neurochem 1991:56:720-723.

54. Rimvall K, Martin DL. Increased intracellular $\gamma$-aminobutyric acid selectively lowers the level of the larger of two glutamate decarboxylase proteins in cultured GABAergic neurons from rat cerebral cortex. J Neurochem 1992;58:158-166. 


\section{Kidney during acute liver failure ${ }^{1}$}

Submitted as: C.H.C. Dejong, N.E.P. Deutz, P.B. Soeters. Renal ammonia and glutamine metabolism during liver insufficiency-induced hyperammonemia in the rat.

\section{ABSTRACT}

Renal glutamine uptake and subsequent urinary ammonia excretion could be an important alternative pathway of ammonia disposal from the body in a situation of diminished hepatic urea synthesis capacity, as e.g. during liver disease, but this pathway has received little attention. Therefore, we investigated this pathway by studying renal glutamine and ammonia metabolism in two models of liver insufficiency-induced hyperammonemia. Mildly hyperammonemic, portacaval shunted rats and severely hyperammonemic rats with acute liver ischemia were compared to their respective appropriate controls, to investigate whether renal ammonia disposal from the body is enhanced during hyperammonemia and to explore the limits of the pathway. For this reason, renal fluxes, urinary excretion and renal tissue concentrations of amino acids and ammonia were measured 24 hours after portacaval shunting, and 2, 4 and 6 hours after liver ischemia induction and in the appropriate controls.

Arterial ammonia and glutamine were increased after portacaval shunting and even more increased during liver ischemia. In contrast to controls, in portacaval shunted rats the kidney net disposed ammonia from the body by diminishing renal venous ammonia release and enhancing urinary ammonia excretion $(\mathrm{P}<0.01)$. However, during liver ischemia, net renal ammonia disposal from the body did not further increase. Renal glutamine uptake was comparable in both hyperammonemic models.

These results indicate that in the rat the kidney plays an important role in ammonia disposal during mild hyperammonemia. However, during severe liver insufficiency induced-hyperammonemia, ammonia disposal capacity appears to be exceeded.

\footnotetext{
'This chapter is an exact copy of the original submission and consequently does not present all data of the experiments. Additional data are available on request.
} 


\section{INTRODUCTION}

Liver disease, whether acute or chronic, is usually accompanied by diminished hepatic urea synthesis capacity, depriving the body of its main route of ammonia detoxification (1). This, combined with the usually existing intra- and extrahepatic portasystemic shunting, leads to hyperammonemia (2), activating altemative pathways of ammonia detoxification. The most important of these alternative pathways probably is the synthesis of the amino acid glutamine from equimolar amounts of glutamate and ammonia (3). It is not generally recognized, however, that glutamine synthesis and subsequent expansion of tissue and plasma free glutamine pools is only a temporary and limited way of ammonia detoxification. To get the ammonia out of the body in a situation of diminished or absent urea synthesis, glutamine has to be transported to and metabolized in the kidney, after which the ammonia can be excreted into the urine.

Arterial glutamine concentrations are generally found to be elevated during liver insufficiency-induced hyperammonemia, a phenomenon thought to be caused by enhanced skeletal muscle glutamine synthesis and release $(1,4-6)$. Because glutamine is the primary substrate for renal ammoniagenesis $(7,8)$, the high glutamine and ammonia concentrations could theoretically enhance renal ammonia disposal, and in the past some data have provided support for this theory (9-14). Thus, although in cirrhotic patients the kidney continued to release ammonia into the circulation $(10,15,16)$, this release decreased at elevated ammonia concentrations (10). Similarly, artificial hyperammonemia in healthy volunteers turned the kidney into an organ of net ammonia uptake from the circulation (12). However, the role of the amino acid glutamine in these metabolic processes is unclear and virtually no literature exists concerning the relation between renal ammonia and glutamine metabolism during liver insufficiency and the related hyperammonemia.

Hyperammonemia is generally thought to be an important factor in the pathophysiology of hepatic encephalopathy $(1,17)$. In the treatment of liver disease, therefore, therapy is focused on keeping arterial ammonia levels low and within narrow limits. Although standard therapy aims at reducing intestinal ammonia liberation, it should be realized that especially the kidney also is an important ammonia producing organ $(18,19)$.

Despite the pivotal role of the kidney in nitrogen disposal and ammonia- and glutamine-related acid-base regulation $(7,15,16,20,21)$, little is known about renal nitrogen metabolism during liver insufficiency and the related hyperammonemia. Therefore, the present experiments were designed to investigate the hypothesis that the kidney plays an important role in ammonia and glutamine metabolism during liver disease. Specifically, we were interested whether renal ammonia disposal from the body is enhanced during liver insufficiency-induced hyperammonemia. To this purpose we compared renal ammonia and glutamine metabolism in mildly hyperammonemic portacaval shunted rats with controls. In addition, we wanted to study whether renal ammonia disposal capacity has an upper limit during liver insufficiency-induced hyperammonemia. To answer this question, rats with severe hyperammonemia, induced by acute liver ischemia, were compared with appropriate controls.

The results show that in the rat, renal ammonia and glutamine metabolism plays an important role in ammonia disposal during mild portacaval shunting-induced hyperammonemia. However, during severe liver ischemia-induced hyperammonemia ammonia disposal 
is not additionally enhanced, providing evidence that the maximal renal ammonia disposal capacity is exceeded.

\section{MATERIALS AND METHODS}

Animals Male Wistar rats $(300 \pm 25 \mathrm{~g}, N=63$ ) housed under standard conditions ( 12 hours light/dark cycle, standard lab chow and water ad libitum) were used throughout. Rats were maintained in accordance with the recommendations of the Guide for the Care and Use of Laboratory Animals, as applied in our institute.

Groups The following experimental groups were studied: PCS CON ALI PortaCaval Shunting.

Laparotomy and manipulations as in PCS rats, but without shunting.

Experimental design (22) Surgery was carried out under ether anesthesia at constant temperature $\left(37 \pm 1^{\circ} \mathrm{C}\right)$. On day $O$, rats were randomized for either construction of a portacaval shunt (by the button method) (PCS-group; $N=55$ ) or sham surgery (CON-group; $N=8$ ). On day $l$, all rats of the CON group and some randomly selected PCS rats (PCS0-group; $N=8$ ) were subjected at $\mathrm{t}=0 \mathrm{~h}$ to the sampling procedures described below. These two groups were compared to address the role of the kidney in mild liver insufficiency-induced hyperammonemia. Also on day 1 , half of the remaining PCS rats were subjected at $\mathrm{t}=0 \mathrm{~h}$ to acute liver ischemia induction by hepatic artery ligation, and sampling 2, 4 or 6 hours later (ALI2, 4 or 6 group; $N=8$ per time point). The remainder underwent a sham procedure at $t=0$ $\mathrm{h}$ in which the hepatic artery was identified but not ligated, and sampling was performed 2, 4 or $6 \mathrm{~h}$ later (PCS2, 4 or 6 group; $N=7$ or 8 per time point). The latter two groups were compared to address the role of the kidney during severe liver insufficiency-induced hyperammonemia.

Miscellaneous Immediately following hepatic artery ligation and every hour thereafter 1 $\mathrm{mL}$ of a $5 \%$ glucose solution was given i.p. to prevent hypoglycemia (23). Rats subjected to hepatic artery identification (PCS2, 4 and 6 groups) received normal saline i.p.. Hourly temperature was measured and level of consciousness was judged using a 6 stage coma scale as described previously (24) (scale 0 to $5 ; 0=$ normal, $5=$ coma). During acute liver ischemia, hepatic encephalopathy progressed to loss of spontaneous righting reflex at $t=6$ hours as described previously (22-24), but no coma was observed. In all other rats no major behavioral changes were observed.

Sampling All rats were overnight fasted before sampling. Rectal temperature was maintained at pre-operative levels: ALI rats $34 \pm 1{ }^{\circ} \mathrm{C}$, other rats $37 \pm 1^{\circ} \mathrm{C}(23)$. Polyethylene catheters (PE-50; Clay Adams, Parsippany, $\mathrm{NJ}$ ) were inserted into the left jugular vein and right carotid artery. A needle bearing micro-catheter (22) was introduced in the left renal vein and cemented in place with cyanoacrylate adhesive. A PE 10 catheter was inserted into the left ureter. For RPF determination, an iso-osmotic, pH adjusted, primed, constant $1 \mathrm{mM}$ PAH (Sigma, St. Louis, MO) infusion was given into the jugular vein $\left(0.08 \mathrm{~mL} \cdot \mathrm{min}^{-1}\right)$, including a 30 min equilibration period to attain steady state arterial PAH concentrations $(22,25,26)$. At steady state, a 15 min urine collection was done on ice, by connecting the free end of the PE 10 ureter catheter airtight to an ice-cooled $30 \mathrm{~cm}$ PE 50 catheter. Urine volume 
(approximately $7 \mu \mathrm{L} \min ^{-1}$ ) was quantified and mixed with a fixed volume of icecold distilled water, divided in portions for amino acid, ammonia and urea determinations and immediately immersed in liquid nitrogen. Next, $1 \mathrm{~mL}$ of blood was withdrawn simultaneously from the renal vein and carotid artery. Finally, the kidney was frozen in liquid nitrogen. Rats were killed by an overdose of the anesthetic. The resulting arterial PAH concentrations $(<70 \mu \mathrm{M})$ are not likely to influence renal ammoniagenesis (27). Also, our urine data in control rats are comparable to studies in which no PAH was used (27-31), providing evidence for the reliability of this urine sampling method.

Biochemical analysis Blood samples were kept on ice during further processing. Hematocrit was obtained with a microfuge. Centrifugations were performed at $4^{\circ} \mathrm{C}$ during 5 $\mathrm{min}$ at $8,800 \mathrm{~g}$. Plasma was put into liquid nitrogen within $20 \mathrm{~min}$. Plasma and tissue samples were stored at $-70^{\circ} \mathrm{C}$ until further processing. Tissue ammonia, amino acids and water content were determined in 5\% sulfosalicylic acid (w/v; SSA) extracts $(22,32)$. Glucose, lactate, ammonia and urea were determined enzymatically (22), and amino acids by HPLC technique as described previously (33). PAH was determined by standard techniques after deacetylation $\left(45 \mathrm{~min}, 100^{\circ} \mathrm{C}\right)(22)$. The coefficient of variance for all determinations was well below $4 \%$ (e.g. $4 \%$ for ammonia, $2 \%$ for glutamine) $(22,33)$.

pH Measurements In some CON, PCS6 and ALI6 rats ( $N=3$ per group) arterial blood was sampled anaerobically for blood $\mathrm{pH}$ at the beginning and end of the $15 \mathrm{~min}$ urine collection. Finally, urine pH was measured in bladder urine. Samples were kept on ice and determined on an ABL520 blood gas analyzer (Radiometer, Copenhagen, Denmark).

Calculations Urea values were corrected for ammonia. Plasma PAH concentrations were calculated using whole blood PAH concentration and hematocrit. RPF ( $\mathrm{mL} \cdot \mathrm{min}^{-1}$ ) was calculated from the amount of PAH infused and renal venous-arterial PAH extraction using standard equations $(22,34,35)$. Fluxes were calculated as RPF times V-A difference (nmole $-100 \mathrm{~g}$ body $\mathrm{wt}^{-1} \cdot \mathrm{min}^{-1}$ per 2 kidneys). Due to technical failure RPF was not available in all animals (see 'Results'). Therefore, the flux data obtained by using the individual RPF were compared with the flux data obtained by using the mean RPF per group per time point. Because the absolute values obtained were similar, and not statistically different (Mann Whitney $U$ test), mean RPF was used for flux calculations. Positive values signify net efflux, negative values reflect net uptake. Urinary excretion was calculated as urine production times urinary concentration and also expressed per two kidneys (nmole $100 \mathrm{~g}$ body $\mathrm{wt}^{-1} \mathrm{~min}^{-1}$ ). Total renal ammoniagenesis was calculated as flux plus urinary excretion. Net renal ammonia disposal from or addition to body ammonia pools was calculated as urinary excretion minus flux. The fraction of ammonia excreted in the urine was calculated as (urinary ammonia excretion/total renal ammoniagenesis) $\cdot 100 \%$.

Statistics (36) Data presented are means \pm SEM, $\mathrm{P}<0.05$ was considered significant. Analysis of variance (ANOVA or ONEWAY procedure, Scheffé comparison), and Mann Whitney- $U$ and Wilcoxon non-parametric tests were used where appropriate.

\section{RESULTS}

Arterial concentrations (Table 1) Arterial concentrations were comparable to those described previously in the same models (22). In ALI rats arterial glucose levels were de 
Table 1. Arterial concentrations.

\begin{tabular}{|c|c|c|c|c|c|c|c|c|}
\hline & CON 0 & PCS O & PCS 2 & PCS 4 & PCS 6 & $A L I 2$ & $\mathrm{ALI} 4$ & ALI 6 \\
\hline $\mathrm{pH}_{\rho \theta 8}$ & $7.386 \pm .033$ & ND & ND & ND & $7.369 \pm 007$ & ND & ND & $7.410 \pm 030$ \\
\hline $\mathrm{pH}_{\text {post }}$ & $7.391 \pm .059$ & ND & ND & ND & $7.406 \pm 025$ & ND & ND & $7.421 \pm 042$ \\
\hline AMM & $51 \pm 8$ & $247 \pm 22^{m}$ & $231 \pm 30$ & $195 \pm 35$ & $169 \pm 21$ & $1044 \pm 69^{m}$ & $820 \pm 37^{\mathrm{m}}$ & $934 \pm 54$ \\
\hline UREA & $8.4 \pm .5$ & $7.7 \pm .4$ & $8.1 \pm .3$ & $8.2 \pm .5$ & $6.5 \pm .3^{\text {prs }}$ & $6.6 \pm .1^{\prime}$ & $5.2 \pm .3^{n}$ & $4.4 \pm .3$ \\
\hline GLC & $8.5 \pm \quad .2$ & $9.5 \pm .8$ & $8.4 \pm .3$ & $9.2 \pm .6$ & $9.1 \pm .4$ & $3.9 \pm .4^{m}$ & $3.7 \pm .7^{\mathrm{m}}$ & $4.3 \pm .4$ chn \\
\hline LAC & $5.0 \pm 1.0$ & $9.3 \pm .9^{\prime}$ & $6.8 \pm 1.1$ & $7.4 \pm .9$ & $7.4 \pm .5$ & $13.2 \pm .4^{m}$ & $8.1 \pm .7$ & $9.4 \pm .7$ cklp \\
\hline GLU & $37 \pm \quad 3$ & $63 \pm 5^{n}$ & $32 \pm 6^{4}$ & $41 \pm 7$ & $45 \pm 7$ & $92 \pm 12^{1}$ & $180 \pm 8^{m}$ & $272 \pm 14^{\operatorname{cthn} p r}$ \\
\hline$\underline{\mathrm{GLN}}$ & $513+40$ & $697 \pm 93^{k}$ & $594 \pm 34$ & $641 \pm 33$ & $689 \pm 53$ & $1852 \pm 71^{\mathrm{m}}$ & $2959 \pm 182^{\text {m }}$ & $3781 \pm 248^{\text {ctinn }}$ \\
\hline
\end{tabular}

Data are means $\pm S E M$ in $\mu \mathrm{M}$, except urea, glucose (GLC), lactate (LAC) in $\mathrm{mM}$, and $\mathrm{pH}$. ND: not determined. Symbols: ANOVA for differences between ALI and PCS; $t=0$ to $t=6 \mathrm{hr}:{ }^{\mathrm{d} P} \mathrm{P}<0.05,{ }^{\mathrm{b}} \mathrm{P}<0.01,{ }^{\mathrm{C}} \mathrm{P}<0.001 ; \mathrm{t}=2$ to $\mathrm{t}=6 \mathrm{hr}:{ }^{\mathrm{d}} \mathrm{P}<0.05,{ }^{\mathrm{o}} \mathrm{P}<0.01$, I $P<0.001 ; t=4$ to $t=6 h r:{ }^{\circledR} P<0.05,{ }^{n} P<0.01, P<0.001$. Mann Whitney- $U$ for differences between $C O N$ and $P C S O$, and between $A L I$ and PCS: ${ }^{x} P<0.05, ' P<0.01,{ }^{m} P<0.001$. Wilcoxon test: "not significantly different from zero. ONEWAY procedure for time

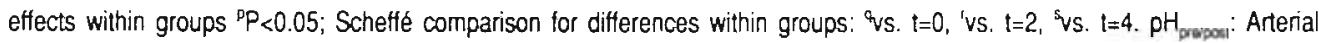
blood sampled at the beginning/end of the 15 min urine collection.

Table 2. Renal metabolite fluxes and RPF.

\begin{tabular}{|c|c|c|c|c|c|c|c|c|}
\hline & CON 0 & PCS 0 & PCS 2 & PCS 4 & PCS 6 & $\mathrm{ALI} 2$ & ALI 4 & ALI 6 \\
\hline $\mathrm{RPF}^{(\mathrm{N})}$ & $3.0 \pm 0.6^{(5)}$ & $2.7 \pm 0.3^{(8)}$ & $4.0 \pm 1.4^{(6)}$ & $3.8 \pm 0.7^{(6)}$ & $2.7 \pm 0.6^{16}$ & $2.5 \pm 0.4^{|6|}$ & $2.7 \pm 0.4^{(7)}$ & $2.3 \pm 0.6^{(6)}$ \\
\hline AMM & $267 \pm 33$ & $-49 \pm 59^{*-1}$ & $186 \pm 75$ & $110 \pm 82^{\circ}$ & $2 \pm 53 *$ & $-28 \pm 136^{\circ}$ & $204 \pm 93$ & $234 \pm 102$ \\
\hline UREA & $598+340^{*}$ & $.766 \pm 287 k$ & $-776 \pm 456^{x}$ & $-1475 \pm 763^{*}$ & $-795 \pm 452^{*}$ & $-696 \pm 336 *$ & $.577 \pm 150$ & $-414 \pm 170^{*}$ \\
\hline GLC & $1774 \pm 575$ & $1162 \pm 998^{*}$ & $985 \pm 732^{*}$ & $64 \pm 814^{*}$ & $-358 \pm 346$ * & $1473 \pm 275$ & $1341 \pm 271$ & $1614 \pm 424^{d x}$ \\
\hline LAC & $-1317 \pm 203$ & $-1345 \pm 526$ & $-1728 \pm 1926$ * & $-2550 \pm 712$ & $-2097 \pm 286$ & $-1714 \pm 594$ & $-1595 \pm 485$ & $-1847 \pm 265$ \\
\hline GLU & $37 \pm 11$ & $15 \pm 20^{*}$ & $56 \pm 16$ & $62 \pm 19$ & $43 \pm 12$ & $48 \pm 26^{*}$ & $34 \pm 13$ & $66 \pm 24$ \\
\hline GLN & $-322+41$ & $-107 \pm 33^{\prime}$ & $-300 \pm 42^{a}$ & $-326 \pm 54^{a}$ & $-163 \pm 30^{p 8}$ & $-180 \pm 79 *$ & $-274 \pm 53$ & $-140 \pm 145^{n}$ \\
\hline
\end{tabular}

Data are means \pm SEM in nmole $\cdot 100 \mathrm{~g}$ body $\mathrm{wt}^{\prime} \cdot \mathrm{min}^{-1}$ per 2 kidneys, except RPF in $\mathrm{mL} \cdot 100 \mathrm{~g}$ body $\mathrm{wt}^{\prime} \mathrm{min}^{-1}$ per 2 kidneys (number of succesfull RPF measurements in parentheses). For significance symbols, see Table 1.

creased compared with portacaval shunted rats subjected to hepatic artery identification (PCS2, 4 and 6 groups), but within the normal range (3-10 mM). Arterial ammonia concentrations were elevated in PCSO compared with CON rats and were grossly elevated in ALI rats compared to PCS2, 4 and 6 controls. Urea levels were comparable in PCSO and CON rats, and, as might be expected, decreased progressively after liver ischemia induction. Arterial concentrations of glutamine were elevated in PCSO compared with CON rats. In ALI rats compared with the PCS2, 4 and 6 groups a progressive increase of glutamine levels to approximately $3800 \mu \mathrm{M}$ at $t=6$ hours was observed. Although arterial $\mathrm{pH}$ at the start of the urine collections was slightly higher in ALI6 than in PCS6 rats, it did not differ from CON rats and was still in the physiological range.

Renal ammonia handling (Table 2-5, Figure 1 \& 2) RPF did not significantly differ between groups (Table 2). In CON rats, net ammonia release into the circulation was observed (Figure 1, right upper panel). In PCS0 rats this ammonia release into the circula- 
Table 3. Urine values.

\begin{tabular}{|c|c|c|c|c|c|c|c|c|c|}
\hline & CON O & PCS 0 & PCS 2 & $\mathrm{PCS} 4$ & PCS 6 & $\mathrm{ALI} 2$ & ALI 4 & ALI 6 & \\
\hline $\mathrm{pH}$ & $6.034 \pm .110$ & ND & ND & ND & $6.028 \pm .028$ & ND & ND & $5.921 \pm .063$ & \\
\hline $\mathrm{VOL}$ & $134 \pm 34$ & $338 \pm 91^{k}$ & $217 \pm 54$ & $229 \pm 66$ & $298 \pm 80$ & $408 \pm 273$ & $464 \pm 145$ & $637 \pm 105$ & 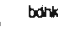 \\
\hline$A M M$ & $113+24$ & $305 \pm 52^{\prime}$ & $235 \pm 37$ & $197 \pm 26$ & $327 \pm 58$ & $252 \pm 30$ & $280 \pm 23^{k}$ & $401 \pm 31$ & bitikps \\
\hline UREA & $266 \pm 108$ & $479 \pm 240$ & $566 \pm 207$ & $327 \pm 102$ & $598 \pm 115$ & $83 \pm 26^{k}$ & $190 \pm 20$ & $223 \pm 42$ & senkep \\
\hline LU & $0.64 \pm 0.18$ & $1.31 \pm 0.28^{k}$ & $0.79 \pm 0.16$ & $0.52 \pm 0.10^{a}$ & $0.61 \pm 0.12^{\circ}$ & $1.89 \pm 0.59^{k}$ & $2.94 \pm 0.73^{\prime}$ & $9.41 \pm 2.17$ & - ctiprs \\
\hline $\mathrm{LN}$ & $0.26 \pm 0.06$ & $0.58 \pm 0.21$ & $0.45 \pm 0.10$ & $0.77 \pm 0.14$ & $0.91 \pm 0.07$ & $1.43 \pm 0.41$ & $2.64 \pm 0.39^{m}$ & $6.29 \pm 1.22$ & stion \\
\hline
\end{tabular}

Data are means $\pm S E M$ in nmole $100 \mathrm{~g}$ body $w^{-1} \cdot \mathrm{min}^{-1}$ (2 kidneys), except VOL $\left(\mu \mathrm{Lhr^{-1 }}\right)$ and $\mathrm{pH}$. ND: not determined. For significance symbols see Table 1.

Table 4. Tissue metabolite concentrations and water content.

\begin{tabular}{|c|c|c|c|c|c|c|c|c|}
\hline & $u$ & PCS 0 & $S 2$ & 4 & $\theta$ & ALI 2 & $\mathrm{ALI} 4$ & ALI 6 \\
\hline $\mathrm{H}_{2} \mathrm{O}$ & & $9 \pm 0.8$ & 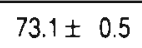 & 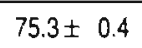 & 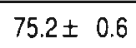 & 5 & $75.6 \pm 0$ & $5.8 \pm 0.5$ \\
\hline AM & 4 & $35 \pm 353^{\prime}$ & $4 \pm 577$ & $6 \pm$ & 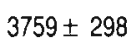 & $879^{1}$ & $9284 \pm 563^{m}$ & $23 \pm$ \\
\hline GIU & $3832+244$ & $37 \pm 272$ & $0-1$ & $3627 \pm 228$ & 5 & 5 & $4943 \pm 485^{\star}$ & 4528 \\
\hline$N$ & $60 \pm 149$ & $1621 \pm 174^{k}$ & $1312 \pm 111$ & $1326 \pm 82$ & $1524 \pm 161$ & $3569 \pm 278^{m}$ & $4745 \pm 236^{m}$ & $8194 \pm 723^{\text {ch }}$ \\
\hline
\end{tabular}

Data are means \pm SEM in $\mu$ mole $k g$ wet $w t^{\prime}$, except tissue waler content $\left(\% \mathrm{H}_{2} \mathrm{O}\right)$. For significance symbols, see Table 1 .

Table 5: Ammonia detoxification

\begin{tabular}{|c|c|c|c|c|c|c|c|c|c|c|c|c|c|c|}
\hline & & & $r$ & & PCS & & PCS & & PCS & & $\mathrm{ALI} 2$ & $\mathrm{ALI} 4$ & ALI 6 & \\
\hline סט & $389+$ & 24 & $65 \pm$ & $25^{\prime}$ & $414 \pm$ & 94 & $7 \pm$ & 79 & $99 \pm$ & 48 & $235 \pm 147^{*}$ & $484 \pm 79$ & $636 \pm 104$ & tor \\
\hline ISP & $-163 \pm$ & 57 & $30 \pm$ & $78^{\prime}$ & $39 \pm$ & $86^{\circ}$ & $58 \pm$ & $84^{\circ}$ & $94 \pm$ & 88 & $99 \pm 97^{\circ}$ & $76 \pm 110^{\prime \prime}$ & $144 \pm 101$ & 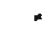 \\
\hline EXCR & $30 \pm$ & 6 & $85 \pm$ & $9^{\prime}$ & $63 \pm$ & 11 & $70 \pm$ & 11 & $90 \pm$ & 11 & $71 \pm 14$ & $67 \pm 11$ & $68 \pm$ & \\
\hline
\end{tabular}

PROD: total renal ammoniagenesis; DISP: ammonia disposal; \%EXCR: percentage of total ammoniagenesis excreted in the urine. Data are means \pm SEM in nmole. $100 \mathrm{~g}$ body $\mathrm{wt}^{-1} \mathrm{~min}^{-1}$ per 2 kidneys, except \%EXCR. For significance symbols, see Table 1.

tion stopped, providing evidence that the kidney plays a role in the adaptation to mild hyperammonemia. Comparison of the ALI group with their PCS controls subjected to hepatic artery identification (PCS2, 4 and 6 groups), revealed no overall significant differences between these groups: ammonia was released into the circulation in both groups at most time points. However, it must be emphasized that in PCS4 and 6 rats, ammonia release was not significantly different from zero, in contrast to ALI4 \& 6 rats. The cause of differences in the time course of ammonia release between these groups is unclear, specifically the ammonia release in the PCS2 group, but might be related to a different response to the laparotomy at $t=0 \mathrm{~h}$ and subsequently elevated arterial glucocorticoid levels (37).

Urinary ammonia excretion $\left(\mathrm{U}_{\mathrm{AMM}} \mathrm{V}\right)$ (Figure 1, left lower panel) was significantly higher in PCSO compared with CON rats. In ALI rats compared with their PCS2, 4 \& 6 


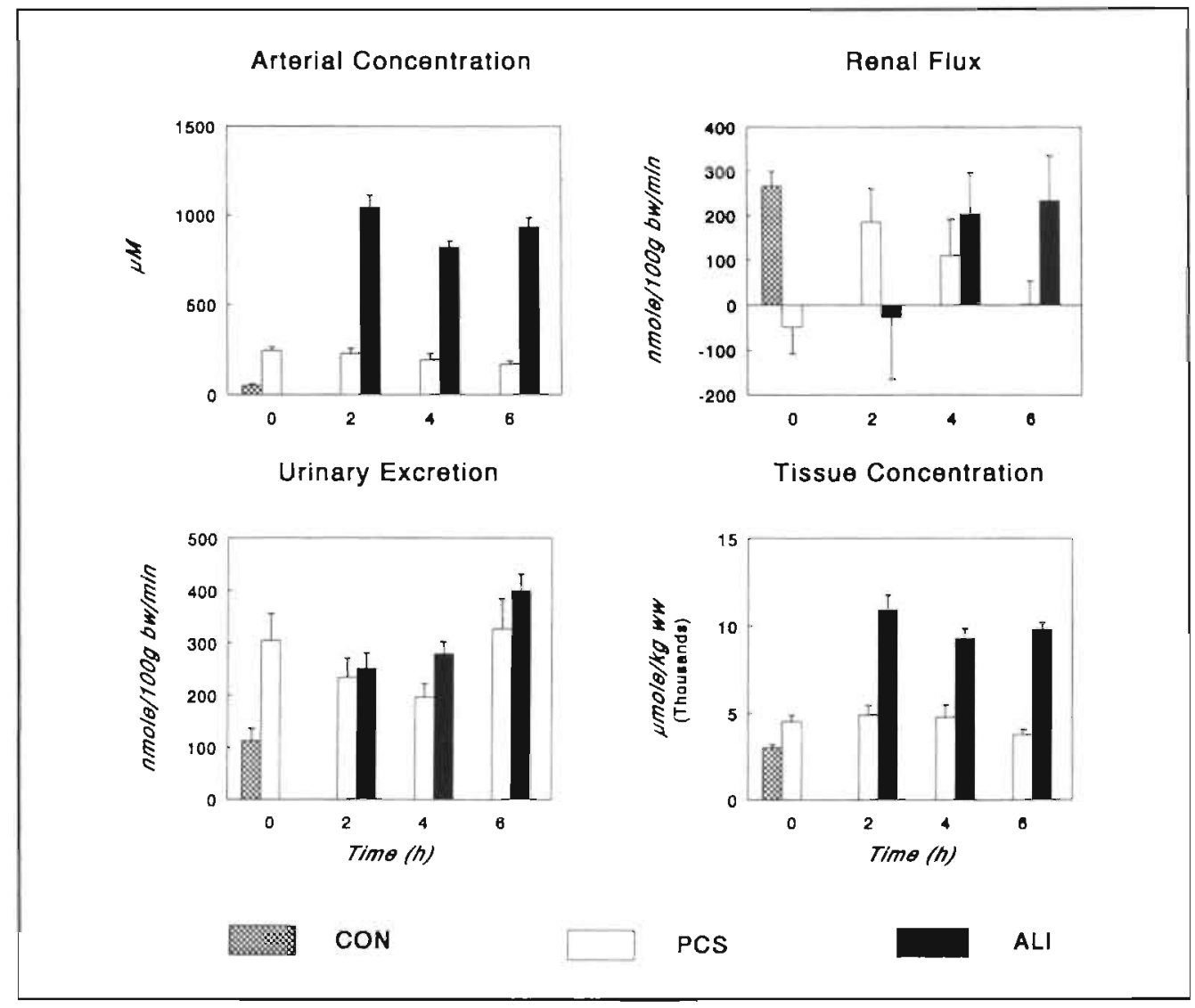

Figure 1. Ammonia: arterial concentrations (left upper panel; $\mu \mathrm{M}$ ), renal fluxes (right upper panel; nmole $100 \mathrm{~g}$ body $\mathrm{wt}^{-1} \cdot \mathrm{min}^{-1}$ per two kidneys), urinary excretion (left lower panel; nmole.100 g body $\mathrm{wt}^{-1} \mathrm{~min}^{-1}$ per two kidneys) and renal tissue concentrations (right lower panel; $\mu$ mole $\mathrm{kg}$ wet $\mathrm{w}^{\prime}$ ) ) in control rats (hatched bars), portacaval shunted rats (open bars) and in rats with acute liver ischemia (filled bars). Data are means $\pm S E M ; N=7$ or 8 per group.

controls, $\mathrm{U}_{\text {AMM }} \mathrm{V}$ was slightly higher. Kidney ammonia (Figure 1, right lower panel) concentrations were elevated in PCSO compared with CON rats, and increased in ALI compared with their PCS2, 4 \& 6 controls.

The overall effect of these changes in renal ammonia fluxes and urinary excretion can be more comprehensively interpreted by looking at the kidney as an organ of net ammonia disposal from or addition to the body ammonia pools (Figure 2, Table 5). In CON rats ammonia release into the renal vein exceeds urinary ammonia excretion and therefore in this group the kidney is an organ of net renal ammonia addition to the body pools (Figure 2, left upper panel; negative disposal). In sharp contrast, in PCS0 rats the kidney reversed to net ammonia disposal from the body ( $\mathrm{P}<0.01, \mathrm{PCS} 0 \mathrm{vs}$. $\mathrm{CON})$. The kidney also proved to be a net ammonia disposal organ in rats subjected to acute liver ischemia (ALI) induction, but in 


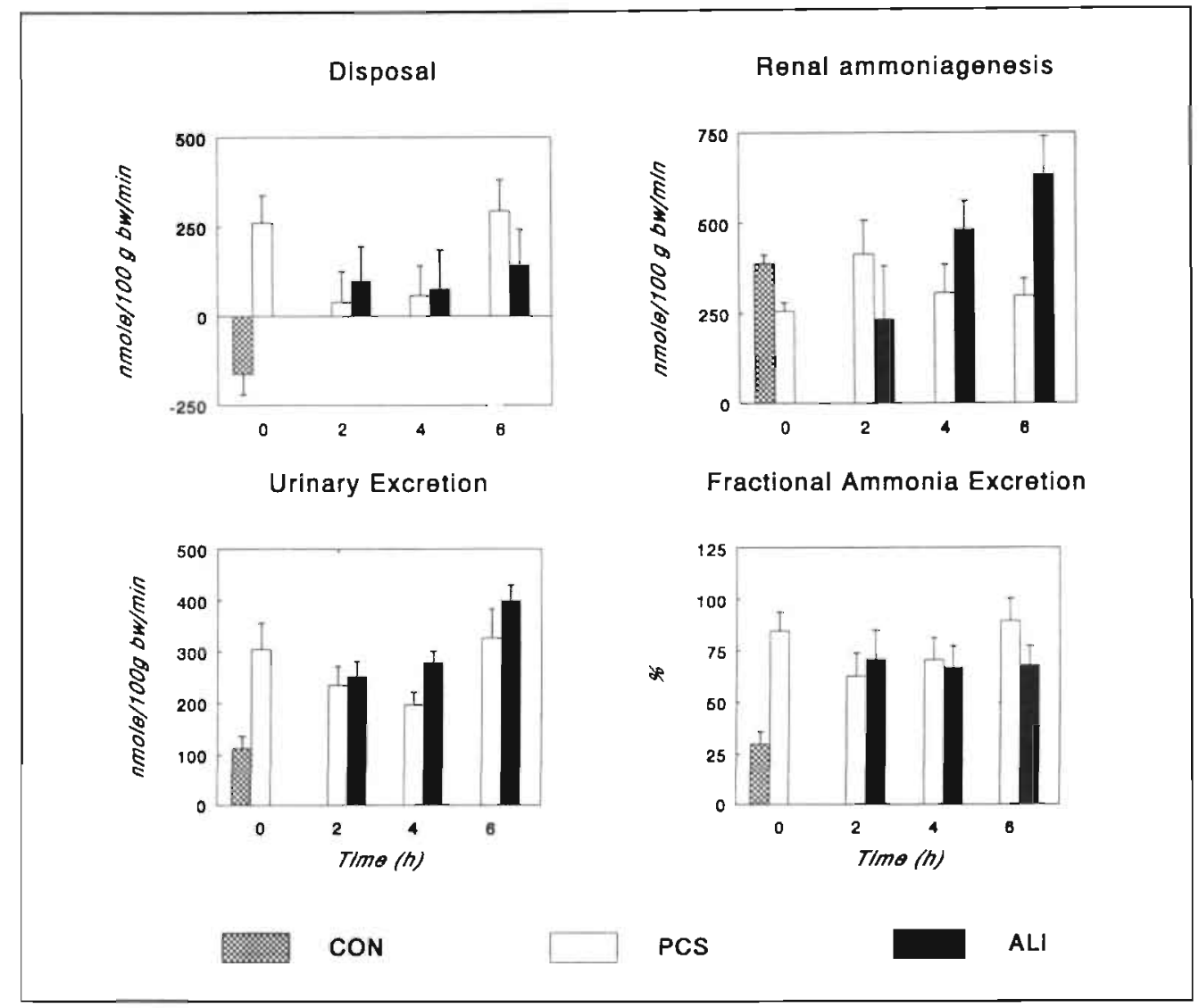

Figure 2. Ammonia deloxification: renal ammonia disposal (urinary excretion minus flux) (left upper panel; nmole $100 \mathrm{~g}$ body $\mathrm{wt}^{\prime 1} \mathrm{~min}^{-1}$ per two kidneys), total renal ammonia genesis (flux + urinary excretion) (right upper panel; nmole $100 \mathrm{~g}$ body $w^{1}{ }^{1}-m_{n}^{-1}$ per two kidneys), urinary excretion (left lower panel; nmole.100 g body $\mathrm{wt}^{-1}-\mathrm{min}^{-1}$ per two kidneys) and fractional ammonia excretion (urinary ammonia excretion expressed as fraction of total ammoniagenesis) (right lower panel; \%) in control rats (hatched bars), portacaval shunted rats (open bars) and in rats with acute liver ischemia (filled bars). Data are means $\pm \mathrm{SEM} ; \mathrm{N}=7$ or 8 per group.

this group ammonia disposal was not significantly different from that observed in their portacaval shunted controls (PCS2, 4, 6 groups). The mechanism of the switch to net ammonia disposal in the PCSO compared to CON group appeared to be a decrease in total renal ammoniagenesis (Figure 2, right upper panel) $(\mathrm{P}<0.01)$ and an increase in the absolute urinary ammonia excretion as well as the fraction of total renal ammoniagenesis excreted in the urine (Figure 2, lower panels) $(\mathrm{P}<0.01)$. In the acute liver ischemia group fractional ammonia excretion and total renal ammoniagenesis were similar to their PCS2, 4 and 6 controls, except for an elevated total renal ammoniagenesis at $t=6 \mathrm{~h}$ in the ALI group.

Renal glutamine handling (Table 2-4, Figure 3) In all groups glutamine was taken up by the kidneys (Figure 3, right upper panel). In PCSO rats glutamine uptake was decreased compared with CON rats, compatible with the decreased total renal ammoniagenesis. No 


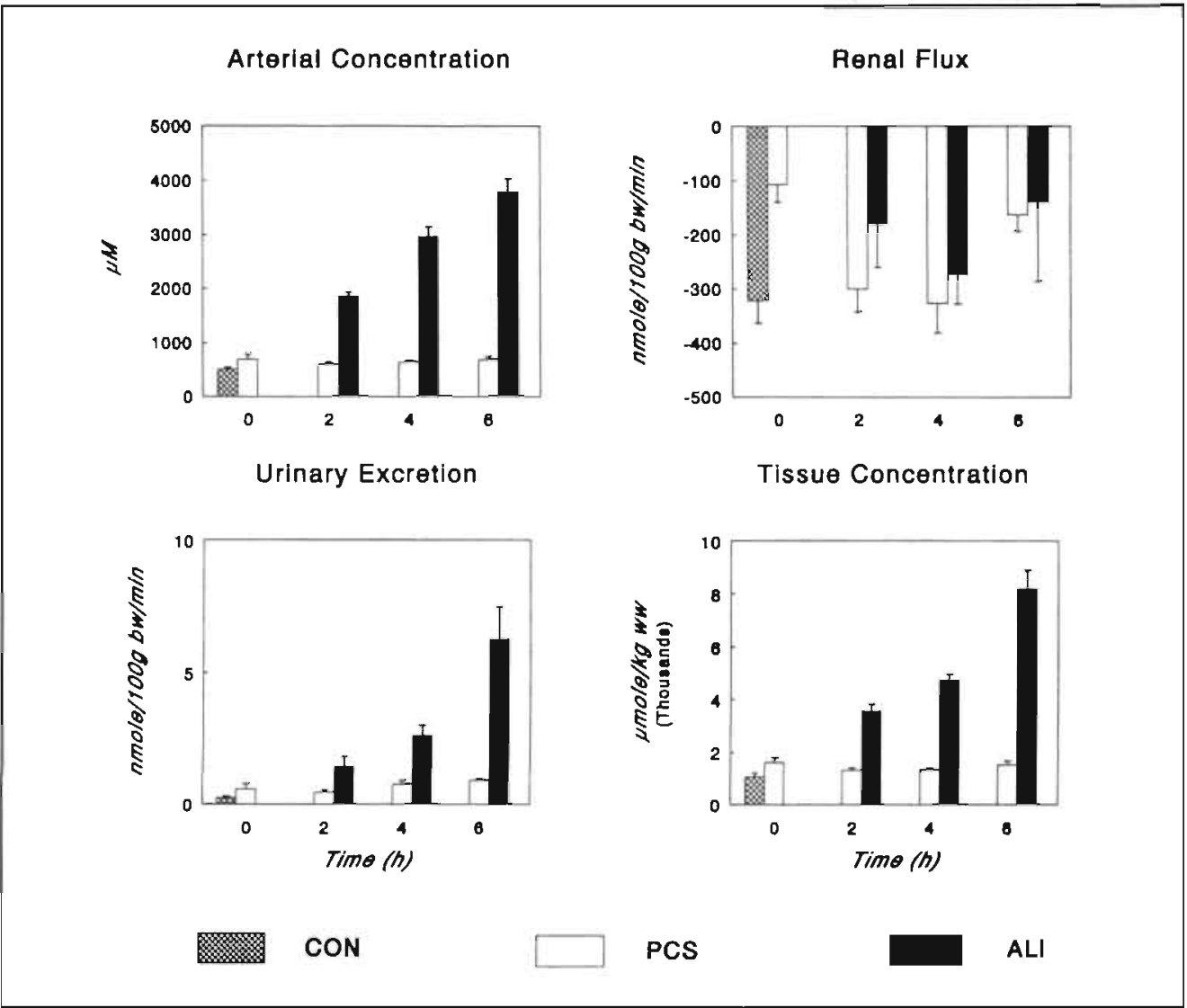

Figure 3. Gilutamine: arterial concentrations (left upper panel; $\mu \mathrm{M}$ ), renal fluxes (right upper panel; nmole.100 $\mathrm{g}$ body $\mathrm{wt}^{-1} \cdot \min ^{-1}$ per two kidneys), urinary excretion (left lower panel; nmole.100 g body wt - min $^{-1}$ per two kidneys) and renal tissue concentrations (right lower panel; $\mu$ mole $\mathrm{kg}$ wet $\mathrm{wt}^{-1}$ ) in control rats (hatched bars), portacaval shunted rats (open bars) and in rats with acute liver ischemia (filled bars). Data are means $\pm S E M ; N=7$ or 8 per group.

differences in glutamine uptake were observed between ALI and PCS2, 4 \& 6 rats, also compatible with the observed total renal ammoniagenesis.

Urinary glutamine excretion $\left(\mathrm{U}_{\mathrm{GLN}} \mathrm{V}\right)$ was comparable in $\mathrm{PCS} 0$ and $\mathrm{CON}$ rats (Figure 3, left lower panel). In ALI rats a progressive increase in $U_{G L N} V$ was observed, reaching levels fivefold elevated at $t=6 \mathrm{~h}$ compared with PCS rats subjected to hepatic artery identification. However, $\mathrm{U}_{\mathrm{GLN}} \mathrm{V}$ still was less than $5 \%$ of renal glutamine uptake in the ALI group. Kidney glutamine (Figure 3, right lower panel) levels were elevated in PCS0 compared with CON rats, and increased in ALI compared with PCS2, 4 \& 6 rats.

Other relevant observations (Table 2-4) Urine production was enhanced in PCS0 rats compared with CON rats, and was increased in ALI compared with PCS2, 4 \& 6 rats. Urea fluxes across the kidney were not significantly different from zero in most groups, due to 
large inter-individual variation. Renal glucose release in ALI rats was significantly higher than in PCS2, 4 \& 6 rats. Renal lactate uptake was similar in all groups. Urine pH was slightly lower in the ALI6 group compared with PCS6 rats, but did not differ from control values. Kidney glutamate concentrations and water content were slightly elevated in ALI compared to PCS2, 4 and 6 rats.

\section{DISCUSSION}

In the present experiments we investigated the role of the kidney in ammonia metabolism during liver insufficiency and the related hyperammonemia. Specifically, we were interested whether the kidney plays an important role in ammonia disposal during hyperammonemia, and whether this ammonia disposal capacity has an upper limit during severe hyperammonemia. To address the first question, we compared renal ammonia handling in portacaval shunted rats with that in sham operated controls. Portacaval shunting in the rat is a wellknown reproducible model for studying various aspects of liver insufficiency, among which the metabolic effects of mild hyperammonemia $(38,39)$. To address the second part of the question, we compared renal ammonia metabolism in rats with acute liver ischemia with that in portacaval shunted rats subjected to hepatic artery identification (ALI versus PCS2 4 \& 6). In analogy to portacaval shunting, the acute liver ischemia model in the rat is very reproducible and it has been used extensively for studying various aspects of severe acute liver insufficiency and hyperammonemia $(22,40-43)$. Theoretically, an adaptive response of the kidney to hyperammonemia could be assumed to require increased renal uptake of blood-derived glutamine, decreased ammonia release into the renal vein and increased $\mathrm{U}_{\mathrm{AMM}} \mathrm{V}(1 \mathrm{l}, 12)$, either apart or in combination.

In the physiological situation, renal ammoniagenesis results from the de-amidation of glutamine mainly by intra-mitochondrial phosphate-dependent glutaminase $(7,9,37,44)$. Only minor quantities $( \pm 10 \%)$ are produced by the brush border membrane-bound $\gamma$ glutamyl transferase in the distal parts of the proximal tubule $(9,45)$. In the physiological situation approximately $70 \%$ of total renal ammoniagenesis is released into the renal vein, the remainder being excreted in the urine (7). The absolute and percentual values found in our study for renal vein ammonia release and $\mathrm{U}_{\mathrm{AMM}} \mathrm{V}$ in CON rats are in agreement with data reported previously $(7,30,31)$.

In CON rats, ammonia release into the renal vein was observed. In PCSO rats, however, ammonia release into the renal vein stopped, and simultaneously $\mathrm{U}_{\mathrm{AMM}} \mathrm{V}$ was increased. These combined changes reversed the kidney from an organ of net ammonia addition to the body pools in CON rats, to an organ of net ammonia disposal in PCSO rats. Another important observation was that total renal ammoniagenesis was diminished in PCS0 compared with CON rats. Thus, the kidney appears to play a very important role in the adaptation to mild hyperammonemia by reducing total ammoniagenesis and increasing the fraction of total ammoniagenesis excreted in the urine, thereby rendering the kidney into an organ of net ammonia disposal. Combining this with data recently reported by our group (22), it can be calculated that the change in renal ammonia handling during portacaval shunting is sufficient to compensate for most of the gut-derived ammonia entering the circulation during portacaval shunting. 
Comparison of rats with acute liver ischemia and their portacaval shunted controls (PCS2, 4 \& 6) revealed that the kidney was an organ of net ammonia disposal in both groups. However, no differences in ammonia disposal were observed, nor were there any significant differences in the fraction of total ammoniagenesis excreted in the urine. From this it appears that in the acute liver ischemia model, during severe hyperammonemia, the capacity of the kidney to enhance ammonia disposal is exceeded. This appears to be in agreement with results obtained in studying the effects of acidosis on renal ammonia excretion $(7,21)$.

Conflicting results have been reported conceming glutamine fluxes across the normal rat kidney $(11,26,27,31,35,46-48)$. Most of these studies show net renal glutamine uptake, but some don't $(11,48)$. The results of the present study provide additional evidence that the normal rat kidney is an organ of net glutamine uptake. In the PCSO group renal glutamine uptake was diminished compared with CON rats, which could contribute to an expansion of the free plasma and tissue glutamine pools (a temporary means of ammonia detoxification). The reduction of renal glutamine uptake was reflected in a reduction in total renal ammoniagenesis in the PCS0 group. Glutamine uptake was similar in ALI rats and in their PCS2, 4 \& 6 controls, compatible with the observation that renal ammonia disposal is not enhanced in ALI rats compared with their PCS2, 4 \& 6 controls. Also, the similar glutamine uptake despite increased arterial glutamine concentrations in the ALI rats provides evidence that renal glutamine uptake and subsequent ammonia disposal is not solely regulated by arterial glutamine levels. The actual regulatory mechanism remains to be determined. Renal tissue glutamine levels were elevated in PCSO compared to CON rats, and showed a progressive increase during liver ischemia compared to their PCS2, 4 \& 6 controls, suggesting that the unchanged $\mathrm{U}_{\mathrm{AMM}} \mathrm{V}$ in $\mathrm{ALI}$ rats is not due to substrate shortage for the phosphate dependent glutaminase pathway. On the contrary, the similar kidney glutamate levels in PCSO and CON rats, and the only marginally increased glutamate levels during acute liver ischemia suggest that the glutaminase pathway is inhibited by the high ammonia levels (3). This would be consistent with the view that (at a given substrate availability) the level of enzyme activity plays a crucial role in regulating ammonia excretion (14) and seems to be the most likely explanation for our observations. Because the kidney contains only minor amounts of glutamine synthetase activity (49), this is not likely to contribute to the observed changes.

A separate note seems to be justified concerning the changes in arterial glutamine concentrations during liver ischemia. In the present study, renal glutamine uptake was comparable in portacaval shunted rats and rats with liver ischemia, suggesting that changes in renal glutamine handling do not explain the observed elevated arterial glutamine levels during acute liver ischemia in the rat (22) (this study). Since recently reported changes in skeletal muscle, intestinal or cerebral cortex glutamine metabolism could not account for this rise either $(22,32)$, the question arises what is the source of the rising glutamine levels. It is attractive to postulate a role for the lung in this respect, because the lung can release considerable quantities of glutamine (50). Alternatively, in the portacaval shunted rat the liver could be an important site of glutamine removal, and the induction of liver ischemia could thereby exclude an important glutamine consuming organ from the circulation, leading to progressive glutamine accumulation. Theoretically, considering the properties of hepatic glutaminase, the high arterial ammonia and glutamine concentrations in portacaval shunted rats, perfusing the liver via a single vessel, could be expected to favor enhanced glutamine removal, but data on this subject are lacking. Similarly, it is difficult to estimate 
the contribution of circulating enzymes, leaked from the liver, to the observed changes in arterial ammonia and glutamine concentrations (51). At the moment, the cause of the increased arterial glutamine concentration remains to be clarified.

The effects of acid-base changes on kidney ammonia and glutamine metabolism have been extensively studied in rats, dogs and humans (for review see e.g. $(7,9,21,52-54$ ). Metabolic acidosis, whether acute or chronic, induces enhanced renal glutamine extraction $(13,35,46,55)$ and increased $\mathrm{U}_{\mathrm{AMM}} \mathrm{V}(13)$ at the expense of urea excretion $(26)$, whereas total urinary nitrogen excretion as ammonia plus urea remains constant $(26,46)$. In contrast, respiratory alkalosis in cirrhotic patients was shown to enhance renal ammonia release into the circulation, thus reducing renal ammonia disposal (15). However, in the present studies, only very minimal $\mathrm{pH}$ changes were observed within the physiological range in agreement with observations by others in the same model (40), making an influence of $\mathrm{pH}$ on the observed changes unlikely. Nonetheless, the changes in fraction of total renal ammoniagenesis excreted in the urine closely resembled those observed in studies during acidosis. Thus, it is well known that during acidosis the fraction of ammonia excreted in the urine rises to $70 \%$, thereby reversing the urine/renal vein ammonia partition ratio (normally $30 / 70$ $(7,21))$. This might suggest that a common pathway is involved in acidosis- and hyperammonemia-induced enhanced urinary ammonia excretion, which does not seem to be mediated by arterial or urine $\mathrm{pH}$. However, the exact mechanism underlying the observed reversal of renal ammonia addition to the body pools in controls, to ammonia disposal during hyperammonemia cannot be deduced from our experiments.

In recent years, it has become increasingly clear that renal ammonia excretion into the urine cannot be explained simply as $\mathrm{pH}$-dependent (the 'diffusion-trapping' system) $(7,44,56,57)$. Instead, several other factors (e.g. tubular urine flow, several ion exchangers and the ammonia countercurrent system) appear to be more important than tubular $\mathrm{pH}$ in the complex regulation of urinary ammonia excretion $(7,44,56-59)$. It is beyond the scope of this paper to discuss these regulatory mechanisms in detail, but if it were possible to influence any of these processes involved in ammonia excretion this could provide a fruitful area for the development of new therapeutics in the treatment of hyperammonemia.

The observed renal lactate and glutamine uptake, and glucose release during ALI is in agreement with the observation in carbon tetrachloride treated rats that the kidney uses lactate and glutamine as a source for gluconeogenesis in a situation in which hepatic lactate utilization and glucose production are inhibited (60). As glucose efflux in our study is maintained during acute liver ischemia, the kidney appears to play an important role in maintaining normoglycemia during impaired liver function, as has been suggested by Katz et al. $(61)$.

In conclusion, the present study shows that during portacaval shunting in the rat, the kidney plays an important role in ammonia disposal during mild hyperammonemia by diminishing total renal ammoniagenesis and enhancing the fraction of renal ammoniagenesis excreted in the urine, thereby turning into an organ of net ammonia disposal. During severe acute hyperammonemia induced by liver ischemia, however, the kidney does not additionally enhance net ammonia disposal from the body, providing evidence that in this situation the capacity of the renal ammonia system to excrete ammonia is exceeded. 


\section{REFERENCES}

1. Zieve L. Pathogenesis of hepatic encephalopathy. Metab Brain Dis 1987;2:147-165.

2. Meijer AJ, Lamers WH, Chamuleau RAFM. Nitrogen metabolism and omithine cycle function. Physiol Rev 1990;70:701-748.

3. Newsholme EA, Leech AR. Biochemistry for the Medical sciences. John Wiley \& Sons, New York, 1983.

4. Gjedde A, Lockwood AH, Duffy TE, Plum F. Cerebral blood flow and metabolism in chronically hyperammonemic rats. Effect of an acute ammonia challenge. Ann Neurol 1978;3:325-330.

5. Lockwood AH, McDonald JM, Reiman RE, Gelbard AS, Laughlin JS, Duffy TE, Plum F. The dynamics of ammonia metabolism in man. Effects of liver disease and hyperammonemia. J Clin Invest 1979;63:449-460.

6. Bessman SP, Bradley JE. Uptake of ammonia by muscle. Its implications in ammoniagenic coma. New Eng J Med 1955;253:1143-1147.

7. Halperin ML, Kamel KS, Ethier JH, Stinebaugh BJ, Jungas RL. Biochemistry and physiology of ammonium excretion, in: The Kidney. Physiology and pathophysiology (edited by Seldin DW \& Giebisch G), Raven Press Ltd., New York, Chapter 76, pp. 2645-2679, 1992.

8. Silbernag|S. The renal handling of amino acids and oligopeptides. Physiol Rev 1988;68:911-1007.

9. Tannen RL. Ammonia metabolism. Am J Physiol 1978;235:F265-F277.

10. Tyor MP, Owen EE, Berry JN, Flanagan JF. The relative role of extremity, liver and kidney as ammonia receivers and donors in patients with liver disease. Gastroenterology 1960;39:420-424.

11. Yablon SB, Relman AS. Glutamine as a regulator of renal ammonia production in the rat. Kidney Int $1977 ; 12: 546$ (abstract).

12. Owen EE, Johnson JH, Tyor MP. The effect of induced hyperammonemia on renal ammonia metabolism. J Clin Invest 1961;40:215-221.

13. Owen EE, Robinson RR. Amino acid extraction and ammonia metabolism by the human kidney during the prolonged administration of ammonium chloride. J Clin Invest 1963;42:263-276.

14. Welboume TC, Weber M, Bank N. The effect of glutamine administration on urinary ammonium excretion in normal subjects and patients with renal disease. J Clin Invest 1972;51:1852-1860.

15. Berry JN, Flanagan JF, Owen EE, Tyor MP. The kidney as a source of blood ammonia in resting and hyperventilated cirrhotics. Clin Res 1959;7:154-155.

16. Owen EE, Tyor MP, Flanagan JF, Berry JN. The kidney as a source of blood ammonia in patients with liver disease: the effect of acetazolamide. J Clin Invest 1960;39:288-294.

17. Record CO. Neurochemistry of hepatic encephalopathy. Gut 1991;32:1261-1263.

18. Imler M, Schlienger J-L, Chabrier G, Comte F. Origine rénale de I'hyperammoniémie provoquée par un régime hyperprotidique chez le rat normal ou porteur d'une stricture portale. Gastroenterol Clin Biol 1983;7:740-745.

19. Imler M, Schlienger J-L, Chabrier G, Simon C. Arterial ammonia changes of renal origin induced in the rat by acid and alkaline diets. Res Exp Med 1986;186:353-363.

20. Souba WW. Interorgan ammonia metabolism in health and disease: A surgeon's view. JPEN 1987;11:569-579.

21. Halperin ML, Ethier JH, Kamel KS. The excretion of ammonium ions and acid base balance. Clin Biochem 1990;23:185-188.

22. Dejong CHC, Kampman MT, Deutz NEP, Soeters PB. Altered glutamine metabolism in rat portal drained viscera and hindquarter during hyperammonemia. Gastroenterology 1992;102:936-948.

23. Deutz NEP, Chamuleau RAFM, de Graaf AA, Bovée WMMJ, de Beer R. In vivo ${ }^{31}$ P-NMR spectroscopy of the rat cerebral cortex during acute hepatic encephalopathy. NMR Biomed 1988;1:101-106.

24. Bosman DK, Deutz NEP, de Graaf AA, van der Hulst RWN, van Eijk HMH, Bovée WMMJ, Maas MAW, Jöming GGA, Chamuleau RAFM. Changes in brain metabolism during hyperammonemia and acute liver failure. Results of a comparative 'H-NMR spectroscopy and biochemical investigation. Hepatology 1990;12:281-290.

25. Dejong CHC, Deutz NEP, Soeters PB. Interorgan nitrogen exchange during prolonged starvation in the rat. J Clin Nutr Gastroenterol 1991;6:176-183.

26. Welbourne TC. Effect of metabolic acidosis on hindquarter glutamine and alanine release. Metabolism 1986;35:614-618.

27. Welbourne TC, Dass PD. Gamma glutamyltransferase contribution to renal ammoniagenesis in vivo. Pflügers Arch 1988;411:573-578. 
28. Farrow NA, Kanamori K, Ross BD, Parivar F. A ${ }^{15} \mathrm{~N}-\mathrm{NMR}$ study of cerebral, hepatic and renal nitrogen metabolism in hyperammonaemic rats. Bioch J 1990;270:473-481

29. Vinay $P$, Allignet E, Pichette C, Warford M, Lémieux G, Gougoux A. Changes in renal metabolite profile and ammoniagenesis during acute and chronic metabolic acidosis in dog and rat. Kidney Int 1980;17:312-325.

30. Warter JM, Imler M, Marescaux C. Chabrier G, Rumbach L, Micheletti G, Krieger J. Sodium valproate-induced hyperammonemia in the rat: role of the kidney. Eur J Pharmacol 1983;87:177-182.

31. Imler M, Chabrier G, Marescaux C, Warter JM. Effects of 2,4-di-nitrophenol on rena] ammoniagenesis in the rat. Eur J Pharmacol 1986; 123:175-179.

32. Dejong CHC, Kampman MT, Deutz NEP, Soeters PB. Cerebral cortex ammonia and glutamine metabolism during liver insufficiency-induced hyperammonemia in the rat. I Neurochem 1992;59:1071-1079.

33. van Eijk HMH, van der Heijden MAH, van Berlo CLH, Soeters PB. Fully automated liquidchromatographic determination of amino acids. Clin Chem 1988;34:2510-2513.

34. Selkurt EE. Measurement of renal blood flow. Meth Med Res 1948;1:191-199.

35. Phromphetcharat $\mathrm{V}$, Welboume TC. Renal glutamine extraction and gut/liver interaction in glutamine homeostasis. Contrib Nephrol 1985;47:9-14

36. Norusis MJ. SPSS/PC+ V3.1 BASE MANUAL for the IBM PC/XT/AT and PS/2. SPSS Inc, Chicago, USA, 1989.

37. Welboume TC. Glucocorticoid control of ammoniagenesis in the proximal tubule. Semin Nephrol 1990); 10:339-349.

38. Mullen KD, McCullough AJ. Problems with animal models of chronic liver disease: Suggestions for improvement in standardization. Hepatology 1989;9:500-505.

39. Ribeiro J, Nordlinger B, Ballet F, Cynober L, Coudray-Lucas C, Baudrimont M, Legendre C, Delelo R, Panis Y. Intrasplenic hepatocellular transplantation corrects hepatic encephalopathy in portacaval shunted rats. Hepatology 1992;15:12-18.

40. Bosman DK, van den Buijs CACG, de Haan JG, Maas MAW, Chamuleau RAFM. The effects of benzodiazepine receptor antagonists and partial inverse agonists on acute hepatic encephalopathy in the rat. Gastroenterology 1991;101:772-781

41. Chamuleau RAFM, Deutz NEP, de Haan JG, van Gool J. Correlation between electroencephalographic and biochemical indices in acute hepatic encephalopathy in rats. J Hepatol 1987:4:299-306.

42. Swain M. Butterworth RF, Blei AT. Ammonia and related amino acids in the pathogenesis of brain edema in acute ischemic liver failure in rats. Hepatology 1992; 15:449-453.

43. Gany R, Swain M, Traber P, Dalcanto M, Butterworth RF, Blei AT. Ammonia induced swelling of rat cerebral cortex slices: implications for the pathogenesis of brain edema in acute hepatic failure. Metab Brain Dis 1989;4:213-223.

44. Good DW, Knepper MA. Mechanisms of ammonium excretion: the role of the renal medulla. Sem Nephrol 1990; 10:166-173.

45. Simon EE. Merli C. Hemdon J, Hamm LL. Contribution of luminal ammoniagenesis to proximal tubule ammonia appearance in the rat. Am J Physiol 1990;259:F402-F407.

46. Welbourne TC, Childress D, Givens G. Renal regulation of interorgan glutamine flow in metabolic acidosis. Am J Physiol 1986;251:R858-R866.

47. Welboume TC. Hepatic glutaminase flux regulation of glutamine homeostasis. Studies in vivo. Biol Chem Hoppe Seyler 1986;367:301-305.

48. Schröck H, Goldstein L. Interorgan relationships for glutamine metabolism in normal and acidotic rats. Am J Physiol 240:E519-E525, 1981.

49. Welbourne TC, Phromphetcharat V, Givens G, Joshi S. Regulation of interorgan glutamine flow in metabolic acidosis. Am J Physiol 1986;250:E457-E463.

50. Welbourne TC. Role of the lung in glutamine homeostasis. Contrib Nephrol 1988;63:178-182.

51. da Fonseca-Wolheim F. Deamidation of glutamine by increased plasma gamma-glutamyltransferase is a source of rapid ammonia formation in blood and plasma specimens. Clin Chem 1990;36:1479-1482.

52. Halperin ML. Ethier JH, Kamel KS. Ammonium excretion in chronic metabolic acidosis: benefits and risks. Am J Kidney Dis 1989;14:267-271.

53. Chobanian MC. Hormonal control of ammonium metabolism: a review. Am J Kidney Dis 1989:14:248-252

54. Schoolwerth AC. Regulation of renal ammoniagenesis in metabolic acidosis. Kidney Int 1991;40:961-973. 
55. Schröck H, Cha CM, Goldstein L. Glutamine release from hindlimb and uptake by kidney in the acutely acidotic rat. Biochem J 1980; 188:557-560.

56. Nagami GT. Ammonia production and secretion by the proximal tubule. Am J Kidney Dis 1989;14:258-261.

57. Hamm LL, Simon EE. Ammonia transpor in the proximal tubule in vivo. Am J Kidney Dis 1989;14:253-257.

58. Kikeri D. Sun A, Zeidel ML, Hebert SC. Cellular $\mathrm{NH}_{4}{ }^{+} / \mathrm{K}^{+}$transport pathways in mouse medullary thick limb of Henle. Regulation by intracellular pH. J Gen Physiol 1992:99:435-461.

59. Nath KA, Hostetter MK, Hostenter TH. The role of ammonia in progressive renal injury, in: Molecular aspects of ammoniagenesis (edited by Endou H, Schoolwerth AC, Baverel G, Tizianello A). Contrib Nephrol Basel, Karger, pp. 78-82, 1991.

60. Faus MJ, Lupianez JA, Vargas A, Sanchez-Medina F. Induction of rat kidney gluconeogenesis during acute liver intoxication by carbon tetrachloride. Biochem J 1978:174:461-467.

61. Katz N, Brinkmann A. Jungermann K. Compensatory increase of the gluconeogenic capacity of rat kidney after partial hepatectomy. Hoppe-Seyler's Z Physiol Chem 1979;360:5I-57. 



\section{Chapter 8}

\section{Muscle during chronic liver failure ${ }^{1}$}

Submitted as: C.H.C. Dejong, N.E.P. Deutz, P.B. Soeters. Muscle amino acid metabolism in two rat models of chronic liver insufficiency: influence of pair-feeding.

\section{ABSTRACT}

The aim of this study was to investigate the role of skeletal muscle in amino acid and ammonia metabolism during chronic liver insufficiency-induced hyperammonemia. Therefore, we studied hindquarter ammonia and amino acid fluxes and muscle tissue concentrations in sham operated, portacaval shunted and portacaval shunted/bile duct ligated rats, 7 and 14 days after surgery, as well as in normal unoperated rats. To reduce nutritional influences, portacaval shunted and sham operated rats were pair-fed to portacaval shunt/biliary obstruction rats.

At days 7 and 14, arterial ammonia was elevated in portacaval shunted and portacaval shunt/biliary obstruction rats. In the latter group, arterial glutamine levels were elevated compared with sham operated controls. No net hindquarter ammonia uptake was observed in any of the groups, despite hyperammonemia in the chronic liver insufficiency groups. At days 7 and 14, hindquarter glutamine release was increased in portacaval shunted and portacaval shunt/biliary obstructed rats, despite similar muscle glutamine levels in sham operated and hyperammonemic groups, suggesting enhanced muscle glutamine synthesis in the latter groups. At days 7 and 14, muscle ammonia levels were increased and muscle glutamate decreased in the hyperammonemic groups, probably indicating glutamate consumption by the enhanced glutamine synthesis. The increased phenylalanine tissue concentrations and efflux in portacaval shunt/biliary obstruction rats suggest that enhanced net muscle protein breakdown, amino acid catabolism and transamination fumish amino acids and ammonia for enhanced glutamine synthesis, rather than ammonia uptake from the

\footnotetext{
'This chapter is an exact copy of the original submission and consequently does not present all data of the experiments. Additional data are available on request. The data described in this chapter were obtained in the same series of experiments as those described in chapter 9; for readability the data concerning muscle and gut metabolism have been described in separate chapters.
} 
blood. These experiments demonstrate that nutritional factors are important in explaining altered muscle metabolism during chronic liver insufficiency.

\section{INTRODUCTION}

Skeletal muscle metabolism is generally thought to play an important role in the well-known alterations in ammonia and amino acid metabolism accompanying chronic liver insufficiency (1-3). This is mainly because total body skeletal muscle mass, apart from its considerable protein content, probably constitutes the quantitatively most important localization of the enzyme glutamine synthetase (2). This enzyme is capable of synthesizing glutamine out of equal amounts of ammonia and glutamate (4), thereby transforming a toxic compound (ammonia) into a non-toxic one (glutamine) (5).

During chronic liver insufficiency, elevated levels of ammonia are often observed, either intermittently or continuously, and hyperammonemic episodes may be precipitated by several causes, such as protein meals and gastrointestinal bleeding (6). These elevations of arterial ammonia levels are generally believed to arise from intestinal ammonia production, escaping hepatic detoxification through the existing intra- and/or extra-hepatic portasystemic shunts $(6,7)$. An additional explanation resides in the fact that total hepatic urea synthesis capacity is reduced during chronic liver insufficiency $(7,8)$.

Ammonia is still considered to be important in the pathogenesis of hepatic encephalopathy, because it exerts toxic effects on the central nervous system $(2,8-10)$. Therefore, ammonia detoxification via glutamine synthesis may become very important during chronic liver insufficiency $(1-3,11)$. However, the quantitative role of muscle in ammonia detoxification in vivo is still unclear $(1,3,12-14)$. Also, in recent studies in our laboratory we did not find increased ammonia uptake or glutamine release by muscle during severely compromised liver function (acute liver ischemia) (15).

Apart from these effects on ammonia and glutamine metabolism, chronic liver insufficiency is accompanied by varying degrees of malnutrition $(6,16)$. Because chronic disease, malnutrition and starvation itself induce enhanced muscle glutamine release (17-20), it is of particular importance to reduce the effects of differences in food intake between the experimental groups in studying ammonia and glutamine metabolism during chronic liver insufficiency.

Therefore, we investigated the role of skeletal muscle in ammonia and glutamine metabolism during chronic liver insufficiency-induced hyperammonemia in the rat under conditions of standardized food intake. The exchange of ammonia and glutamine (and several other amino acids) across the hindquarter ( $\approx$ muscle) as well as muscle tissue concentrations were studied in two models of chronic liver insufficiency: portacaval shunting, and portacaval shunting plus bile duct ligation, as well as in appropriate controls. Although portacaval shunting is a well-known reproducible model of chronic liver insufficiency-induced hyperammonemia and hepatic encephalopathy (16,21), portacaval shunting combined with common bile duct ligation probably provides a more reliable model for chronic hyperammonemia and hepatic encephalopathy (22). This model combines the effects of portasystemic shunting with the occurrence of biliary cirrhosis of the liver, presumably leading to a more pronounced degree of liver failure. Bile duct ligation and resulting hyperbilirubinemia $(23,24)$ also simulate the impaired bile excretion and the 
probably related immune system dysfunction (23-25) in patients with chronic cirrhosis of the liver.

The present experiments suggest that nutritional factors are important in explaining altered muscle metabolism during chronic liver insufficiency. Enhanced net muscle protein breakdown, amino acid catabolism and transamination appear to furnish the amino acids and ammonia for enhanced glutamine synthesis, rather than ammonia uptake from the blood.

\section{MATERLALS AND METHODS}

Animals Male Wistar rats ( $\mathrm{n}=56,300 \pm 25 \mathrm{~g}$ ) were used throughout. Rats were housed under standard conditions ( $12 / 12 \mathrm{~h}$ light/dark cycle) and received standard food and water ad libitum until entering the experiments i.e. until surgery. Rats were maintained and humanely cared for according to the recommendations of the Guide for the Care and Use of Laboratory Animals, as applied in our institute.

Groups Four groups were studied: 1 PCSBDL group. Portacaval shunting (PCS) plus bile duct ligation (BDL) in one surgical session. 2 PCS-PF group. Portacaval shunting. $3 P F$ group. Laparotomy and manipulations as in PCS-PF rats, but without shunting (sham operation). 4 NORM group. Sampling without previous surgery; normal control rats, receiving food ad libitum.

Before surgery, rats were randomly assigned to one of the groups. Surgical procedures were of equal duration in PCS-PF and PF rats and their individual PCSBDL mate. After resuscitation from surgery, PCSBDL, PCS-PF and PF rats were placed in metabolic cages. PCSBDL rats had free access to standard pellet food. In these rats, daily food intake was recorded and this amount was administered to fixed PCS-PF and PF mates to eliminate effects of differences in food intake (pair-feeding: PF). In the PCSBDL, PCS-PF and PF groups blood was sampled 7 or 14 days after surgery (see below). Body weight was measured at the time intervals indicated in the figures. Water was administered ad libitum in all groups.

Behavior Before entering the experiments and before sampling, behavior of all rats was studied during a $5 \mathrm{~min}$ period. Specific attention was paid on appearance, presence of lethargy, spontaneous locomotor activity and exploratory behavior, presence of tiptoe gait, toeing out and arched back, reaction to tail pinching and presence of ataxia. Since the PCSBDL rats were jaundiced, this staging procedure could not be performed blinded.

Surgery Surgery and sampling were performed under ether anesthesia. Portacaval shunting was performed by the button technique $(15,26)$. Biliary obstruction was achieved by ligating the common bile duct near the liver and near the duodenum and removing the piece between the ligatures. In PCS-PF and PF rats, the bile duct was manipulated, but not dissected, to avoid stenosis.

Sampling Prior to sampling, rats were fasted ovemight. Sampling procedures were performed as described elsewhere $(15,17,27)$. Briefly, rats were anesthetized 30 min before sampling and the abdominal aorta and inferior caval vein were cannulated (both just above the iliac bifurcation) with needle bearing micro-catheters, fixed in place with cyanoacrylate adhesive. The right common carotid artery was catheterized with polyethylene tubing (PE 
Table 1. Behavior

\begin{tabular}{|c|c|c|c|c|c|c|c|c|c|c|}
\hline & \multirow{2}{*}{$\begin{array}{l}\text { DAY O } \\
\text { NORM }\end{array}$} & \multicolumn{4}{|c|}{ DAY 7} & \multicolumn{5}{|c|}{ DAY 14} \\
\hline & & PF & PCS-PF & PCSBDL & & PF & PCS-PF & & PCSBDL & \\
\hline 'Gerbil' appearance & $0 / 10$ & $4 / 8$ & $5 / 8$ & $8 / 8$ & a & $1 / 8$ & $5 / 8$ & & $6 / 6$ & t \\
\hline Toeing out (hindlimbs) & $0 / 10$ & $0 / 8$ & $0 / 8$ & $6 / 8$ & n & $0 / 8$ & $6 / 8$ & $f$ & $6 / 6$ & $s$ \\
\hline Tiptoe gait & $0 / 10$ & $0 / 8$ & $1 / 8$ & $3 / 8$ & & $0 / 8$ & $1 / 8$ & & $6 / 6$ & su \\
\hline 'Duck' gait & $0 / 10$ & $0 / 8$ & $1 / 8$ & $3 / 8$ & & $0 / 8$ & $2 / 8$ & & $6 / 6$ & su \\
\hline Arched back & $0 / 10$ & $0 / 8$ & $1 / 8$ & $3 / 8$ & & $0 / 8$ & $3 / 8$ & & $5 / 6$ & ' \\
\hline Ataxia & $0 / 10$ & $0 / 8$ & $0 / 8$ & $2 / 8$ & & $2 / 8$ & $6 / 8$ & & $6 / 6$ & - \\
\hline
\end{tabular}

All items were scored on a dichotomic basis i.e. present or absent. $X N$ in the table indicates that the item was $X$ times present in $\mathrm{N}$ rats. Fischer's one-tailed exact test: vs. $P F{ }^{a} P<0.05,{ }^{\prime} P<0.01,{ }^{3} P<0.001$; vs. $P C S-P F{ }^{\prime} P<0.05,{ }^{4} P<0.01,{ }^{~} P<0.001$.

Table 2. Arterial concentrations.

\begin{tabular}{|c|c|c|c|c|c|c|c|c|c|c|}
\hline & DAY 0 & & DAY 7 & & & & & DAY 14 & & \\
\hline & NORM & PF & PCS-PF & & PCSBDL & & PF & PCS-PF & PCSBDL & \\
\hline ILI & $1.3 \pm 0.1$ & $1.2 \pm 0.1$ & $2.4 \pm 0.4$ & $n$ & $119.9 \pm 7.6$ & ik & $1.3 \pm 0.1$ & $2.6 \pm 0.3$ & an $112.9 \pm 7.3$ & \\
\hline LC & $7.9 \pm 0.3$ & $9.4 \pm 0.7$ & $8.9 \pm 0.5$ & & $7.1 \pm 0.2$ & ok & $9.4 \pm 0.4$ & $8.0 \pm 0.4$ & $7.9 \pm 0.3$ & $\infty$ \\
\hline$A C$ & $3.4 \pm 0.3$ & $4.5 \pm 1.0$ & $9.0 \pm 0.7$ & 8 & $10.5 \pm 0.8$ & $n$ & $5.3 \pm 0.7$ & $9.2 \pm 0.9$ & $9.9 \pm 0.8$ & \\
\hline MM & $59 \pm 6$ & $59 \pm 12$ & $199 \pm 19$ & $n$ & $196 \pm 20$ & 1 & $68 \pm 7$ & $181 \pm 26$ & $168 \pm 25$ & \\
\hline JREA & $7.7 \pm 0.4$ & $7.1 \pm 0.4$ & $6.1 \pm 0.4$ & & $6.1 \pm 0.2$ & 9 & $7.7 \pm 0.2$ & $6.4 \pm 0.3$ & $6.3 \pm 0.3$ & \\
\hline iLU & $82 \pm 8$ & $70 \pm 7$ & $79 \pm 14$ & & $55 \pm 5$ & $\theta$ & $61 \pm 7$ & $66 \pm 7$ & $40 \pm 4^{\log }$ & \\
\hline SN & $45 \pm 2$ & $40 \pm 3$ & $61 \pm 5$ & $n$ & $67 \pm 5$ & $n$ & $39 \pm 2$ & $58 \pm 4^{\circ}$ & $53 \pm 2$ & $\infty n$ \\
\hline LN & $554 \pm 12$ & $592 \pm 20^{m}$ & $644 \pm 23$ & & $713 \pm 40$ & $n$ & $604 \pm 24$ & $609 \pm 17$ & $599 \pm 27$ & up \\
\hline LA & $301 \pm 13$ & $264 \pm 21$ & $393 \pm 46$ & $\bullet$ & $409 \pm 39$ & h & $263 \pm 25$ & $385 \pm 19$ & $333 \pm 21$ & \\
\hline UET & $42 \pm 2$ & $35 \pm 2^{m}$ & $43 \pm 4$ & & $46 \pm 3$ & $\theta$ & $36 \pm 2$ & $44 \pm 2$ & $37 \pm 2$ & $a p$ \\
\hline TRP & $52 \pm 3$ & $46 \pm 3$ & $53 \pm 5$ & & $45 \pm 2$ & & $52 \pm 2$ & $56 \pm 2$ & $44 \pm 2$ & lok \\
\hline YS & $369 \pm 21$ & $330 \pm 17$ & $334 \pm 20$ & & $388 \pm 22$ & & $286 \pm 9^{p}$ & $326 \pm 17$ & $292 \pm 17$ & $m$ \\
\hline TYR & $72 \pm 5$ & $65 \pm 6$ & $87 \pm 5$ & $\theta$ & $119 \pm 7$ & 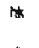 & $66 \pm 4$ & $94 \pm 5$ & $103 \pm 5$ & 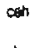 \\
\hline PHE & $66 \pm 3$ & $60 \pm 3$ & $80 \pm 5$ & n & $103 \pm 5$ & $\star$ & $65 \pm 4$ & $80 \pm 4^{\circ}$ & $82 \pm 3$ & \\
\hline$B C A A$ & $404 \pm 17$ & $394 \pm 26$ & $337 \pm 37$ & & $318 \pm 27$ & 0 & $394 \pm 18$ & $354 \pm 20$ & $273 \pm 17$ & $d k$ \\
\hline$\alpha A N$ & $3016 \pm 84$ & $2831 \pm 91$ & $3201 \pm 170$ & & $3593 \pm 156$ & 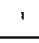 & $2835 \pm 95$ & $3069 \pm 104$ & - $\quad 3030 \pm 106$ & \\
\hline
\end{tabular}

Dala are expressed as means $\pm S E M$ in $\mu \mathrm{M}$, except urea, glucose (GLC) and lactate (LAC) in mM. BCAA - branched-chain amino acids LEU, ILE and VAL. Symbols: ANOVA for group effects; $t=7$ to $t=14$ days: vs. $P F{ }^{4} P<0.05,{ }^{b} P<0.01,{ }^{c} P<0.001 ; v s$. PCS-PF ${ }^{d} P<0.05,{ }^{8} P<0.01,{ }^{P}<<0.001$. Mann-Whitney $U$ for differences between groups: vs. $P F$ gP $<0.05,{ }^{h} P<0.01,{ }^{\prime} P<0.001 ; v s$. PCS-PF $P<0.05$, ${ }^{k}<<0.01, ' P<0.001$; vs. NORM ${ }^{m} P<0.05,{ }^{n} P<0.01,{ }^{\circ} P<0.001$. Wilcoxon test: "not significantly different from zero. ONEWAY procedure for time effects within groups ${ }^{\circ} \mathrm{P}<0.05$.

50). For flow determinations, after a priming dose, a $5 \mathrm{mM}$ isomolaric, $\mathrm{pH}$ adjusted paraaminohippuric acid solution was continuously infused in the abdominal aorta ( $25 \mu \mathrm{L} \mathrm{min}{ }^{-1}$ ), allowing for a $20 \mathrm{~min}$ period to attain steady state concentrations $(28,29)$. Blood was slowly and simultaneously aspirated from the inferior caval vein and carotid artery and put in heparinized tubes on ice. Finally, the right gastrocnemius muscle was 
Table 3. Hindquarter metabolite fluxes and plasma flows.

\begin{tabular}{|c|c|c|c|c|c|c|c|c|}
\hline & DAYO & & DAY 7 & & & & DAY 14 & \\
\hline & NORM & PF & PCS-PF & PCSBDL & & $\mathrm{PF}$ & PCS-PF & PCSBDL \\
\hline LOW & $2.6 \pm 1.1$ & $1.9 \pm 0.4$ & $3.5 \pm 0.6$ & $3.9 \pm 1.3$ & & $5.5 \pm 0.8^{\infty}$ & $5.3 \pm 2.8$ & $3.3 \pm 2.3$ \\
\hline AMM & $-10 \pm 15$ & $1 \pm 9^{\circ}$ & $6 \pm 26^{*}$ & $49 \pm 46$ & * & $-31 \pm 23$ & $12 \pm 53$ & $15 \pm 26$ \\
\hline ṠLU & $-43 \pm 9$ & $-18 \pm 6^{m}$ & $-44 \pm 25$ & $-23 \pm 8$ & & $-61 \pm 12^{D}$ & $-65 \pm 19$ & $-16 \pm 7$ \\
\hline ISN & $16 \pm 3$ & $16 \pm 4$ & $25 \pm 7$ & $39 \pm 7$ & ${ }^{n}$ & $40 \pm 9^{D}$ & $21 \pm 7$ & $30 \pm 6$ \\
\hline$N$ & $114 \pm 30$ & $141 \pm 29$ & $300 \pm 69$ & \pm 54 & : & $261 \pm 79$ & $394 \pm 55$ & $358 \pm 60$ \\
\hline ALA & $94 \pm 37$ & $101 \pm 25$ & $33 \pm 59=$ & $114 \pm 57$ & * & $220 \pm 81$ & $7 \pm 31^{\infty}$ & $29 \pm 37$ \\
\hline IET & $4 \pm 3^{*}$ & $8 \pm 2$ & $5 \pm 6$ & $26 \pm 6$ & h & $9 \pm 5^{-}$ & $3 \pm 4^{\prime \prime}$ & $16 \pm 4$ \\
\hline$R P$ & $-10 \pm 5^{*}$ & $-7 \pm 3$ & $-1 \pm 2$ & $-5 \pm 4$ & * & $-26 \pm 7^{p}$ & $-39 \pm 13^{p}$ & $-20 \pm 2$ \\
\hline YS & $59 \pm 35$. & $40 \pm 20^{\prime}$ & $70 \pm 39$ & $137 \pm 20$ & $n$ & $161 \pm 44^{\circ}$ & $54 \pm 32 *$ & $114 \pm 43$ \\
\hline YF & $2 \pm 4$ & $2 \pm 2^{*}$ & $10 \pm 7^{\prime \prime}$ & $14 \pm 6$ & $\mathfrak{g}$ & $-11 \pm 6{ }^{\infty}$ & $.4 \pm 9^{\prime \prime}$ & $-3 \pm 7$ \\
\hline HE & $5 \pm 5^{-}$ & $9 \pm 3$ & $17 \pm 8$ " & $20 \pm 3$ & $\mathrm{~h}$ & $0 \pm 8$. & $5 \pm 7$. & $17 \pm 6$ \\
\hline BCAA & $-26 \pm 18$ & $-3 \pm 10^{m}$ & $-1 B \pm 22$ & $-24 \pm 19$ & " & $-179 \pm 35^{D}$ & $-99 \pm 18^{\circ}$ & $-2 \pm 13^{-\infty}$ \\
\hline XAN & $320 \pm 151^{*}$ & $445 \pm 127$ & $569 \pm 281$ & $1056 \pm 122$ & ${ }^{n}$ & $654 \pm 319^{*}$ & $473 \pm 130$ & $697 \pm 177$ \\
\hline
\end{tabular}

Data are means \pm SEM in nmole $100 \mathrm{~g}$ body $\mathrm{wt}^{-1} \mathrm{~min}^{-1}$, except plasma flows in $\mathrm{mL} \cdot 100 \mathrm{~g}$ body $\mathrm{wt}^{-1} \cdot \mathrm{min}^{-1}$. For significance symbols, see Table 2.

Table 4. Gastrocnemius muscle tissue concentrations and water content.

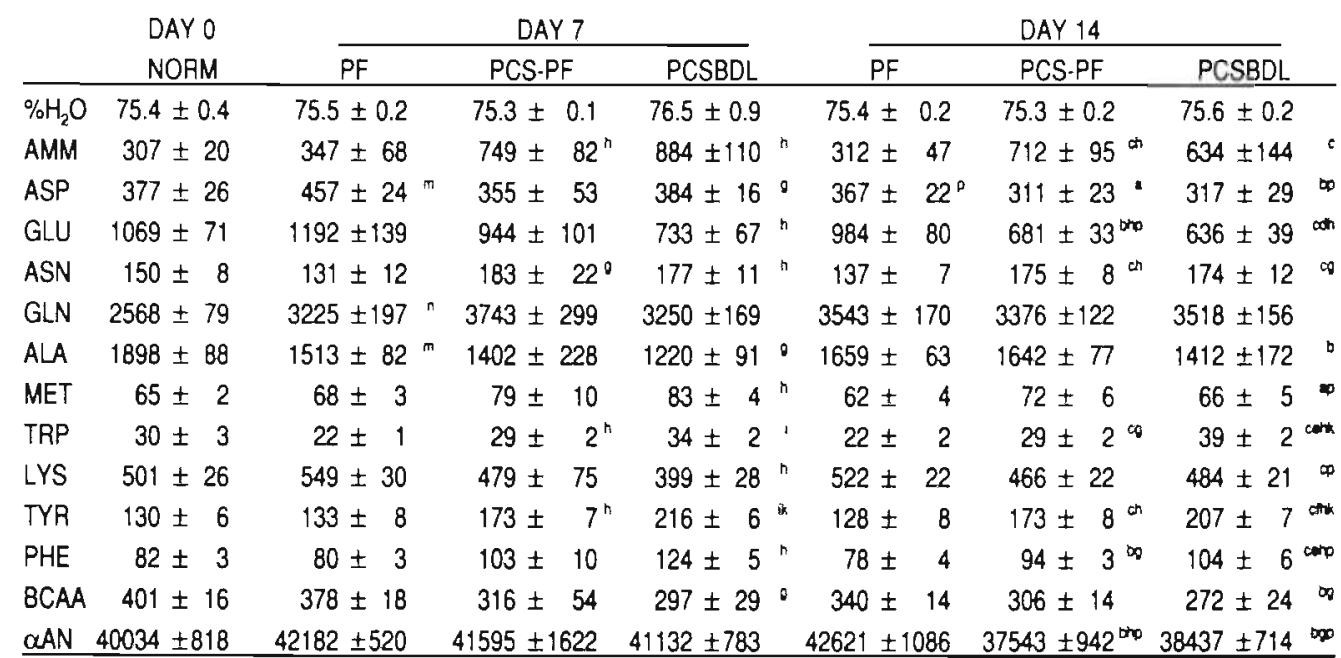

Data are means \pm SEM in $\mu$ mole $k g$ wet weight ${ }^{1}(w w)$, except tissue water content $\left(\% \mathrm{H}_{2} \mathrm{O}\right)$. For significance symbols, see Table 2.

excised and frozen in liquid nitrogen (15). Rats were killed by an overdose of the anaesthetic. The above-described procedures permit simultaneous determination of blood flow and arteriovenous concentration difference ( $=$ flux) across the hindquarter ( $\approx$ muscle), as well as muscle tissue concentrations $(15,17,27)$. 
Analysis Blood samples were kept on ice during processing. Plasma for amino acids and ammonia was obtained by whole centrifugation. Centrifugations were performed at $4^{\circ} \mathrm{C}$ during $5 \mathrm{~min}$ at $8,800 \mathrm{~g}$. Hematocrit was obtained with a microfuge. Plasma and tissue was stored at $-70^{\circ} \mathrm{C}$. For tissue ammonia and amino acid determinations, $5 \%(\mathrm{w} / \mathrm{v})$ sulfosalicylic acid extracts were prepared (15). Tissue water content was determined as described previously (28). Ammonia, lactate and glucose were determined enzymatically (15), amino acids by HPLC technique (30). PAH was determined as described previously (15). Total bilirubin was determined in plasma using a commercial kit (Roche, Basel, Switzerland). The coefficient of variance for all determinations was below 4\% $(15,30)$.

Calculations Plasma PAH concentration was calculated from whole blood PAH concentrations using the hematocrit. Plasma flow was calculated using the formula $\mathrm{F}_{\mathrm{HQ}}=\mathrm{I} /\left([\mathrm{PAH}]_{\mathrm{ICV}}-[\mathrm{PAH}]_{\mathrm{A}}\right)$. In this formula, $\mathrm{F}_{\mathrm{HQ}}$ is the hindquarter plasma flow ( $\mathrm{mL} \mathrm{min}^{-1}$ ), $\mathrm{I}$ is the infused PAH $\left(\mu\right.$ mole $\left.\mathrm{min}^{-1}\right)$, and $[\mathrm{PAH}]_{1 \mathrm{CV} . \mathrm{A}}$ is the plasma PAH concentration $(\mu \mathrm{M})$ in the inferior caval vein and carotid artery, respectively.

Hindquarter fluxes (nmole-100 g body weight ${ }^{-1}$ $\mathrm{min}^{-1}$ ) were calculated as plasma flow times inferior caval venous-arterial concentration difference. Positive figures mean net efflux, negative values reflect net uptake.

Urea values were corrected for ammonia. $\alpha$-Amino nitrogen was calculated as the sum of the $\alpha$-amino groups of all individual amino acids measured (30).

Statistics Data presented are means \pm SEM, $\mathrm{P}<0.05$ was considered significant. Statistics were performed using the SPSS/PC+ Statistical Software Package, version 3.1 (SPSS Inc., Chicago, IL., USA). Analysis of variance was used to test for group effects (ANOVA) and time effects within groups (ONEWAY). The Mann-Whitney $U$ non-parametric test was used for differences between groups at specific time points. Wilcoxon's nonparametric test was used for differences from zero. Fischer's exact test was used to test for differences in encephalopathy stages (one-tailed, binomial testing). Significances are indicated in Tables $1-4$.

\section{RESULTS}

General aspects PCSBDL rats appeared to be more encephalopathic, as evidenced by abnormal gait, ataxia, and decreased spontaneous locomotor activity and exploratory behavior (Table 1). Food intake (Figure 1, upper panel) was similar in PCSBDL, PCS-PF 
and $\mathrm{PF}$ rats during the whole experiment and no significant differences in body weight were observed between the groups (Figure 1, lower panel). Also, no signs of systemic or local inflammation or impaired wound healing were observed in the PCSBDL group. Total bilirubin levels (Table 2) were increased in PCS-PF compared to $\mathrm{PF}$ and in PCSBDL compared to PCS-PF rats. Glucose levels were decreased and lactate levels were elevated in both hyper-ammonemic groups compared to PF rats (Table 2).

Ammonia (Figure 2; Table 2-4) Arterial ammonia levels were equally elevated in PCSBDL and PCS-PF rats at both day 7 and 14, but hindquarter ammonia fluxes were not significantly different from zero in any group. Yet, gastrocnemius muscle ammonia concentrations were twofold elevated in both hyperammonemic groups.

Glutamine (Figure 3; Table 2-4) At day 7, arterial glutamine concentrations were higher in $\mathrm{PF}$ than in NORM rats, and in PCSBDL compared to PF rats, but no differences were observed at day 14. Hindquarter glutamine efflux was overall increased in the hyperammonemic groups compared with the $\mathrm{PF}$ group $(\mathrm{P}<0.05$; ANOVA day 7 to 14), a difference mainly caused by enhanced muscle glutamine release at day 7 , since no major differences were observed at day 14. Simultaneously, muscle glutamine levels were similarly elevated at all time points in all operated groups compared with normal rats, and no differences were observed between PF, PCS-PF and PCSBDL rats. From these data it can be concluded that muscle glutamine production is enhanced, particularly in the hyperammonemic groups at day 7. Also, muscle glutamine levels appear to be unrelated to arterial or skeletal muscle ammonia concentrations, suggesting an important contribution of nutritional status. The enhanced muscle glutamine production in the hyperammonemic groups makes it of special interest to consider some of the key amino acids in the glutamine biosynthetic pathway: glutamate and the branched chain amino-acids.

Glutamate (Figure 4; Table 2-4) In PCSBDL rats arterial glutamate was diminished compared with both the PCS-PF and PF group. Glutamate was taken up by the hindquarter in all groups at all time points, but hindquarter glutamate uptake was clearly reduced in PCSBDL compared to PCS-PF and PF rats. Despite similar skeletal muscle glutamine levels in PCSBDL, PCS-PF and PF rats, muscle glutamate was 
decreased in the hyperammonemic groups only, suggesting that muscle glutamate levels are related to muscle ammonia levels rather than to nutritional status.

BCAA (Figure 5; Table 2-4) Arterial BCAA levels were decreased in both chronic liver insufficiency groups compared to PF at both time points. BCAA uptake by the hindquarter was observed in normal overnight fasted control rats, but at day 7 had decreased to values not significantly different from zero. At day 14 , BCAA uptake was again observed in PF and PCS-PF, but not in PCSBDL rats. Skeletal muscle BCAA levels were decreased in PCSBDL rats, compared with PF rats, suggesting enhanced BCAA consumption by muscle, which after transamination could provide glutamate for glutamine synthesis.

Phenylalanine (Figure 6; Table 2-4) Arterial as well as tissue phenylalanine were elevated in both chronic liver insufficiency groups, more prominent in PCSBDL rats. Simultaneously, hindquarter phenylalanine efflux was augmented in PCSBDL compared with PF rats, suggesting enhanced net muscle protein breakdown, which could provide also substrates for enhanced glutamine synthesis.

\section{DISCUSSION}

The present experiments were designed to study skeletal muscle ammonia and amino acid metabolism during chronic liver insufficiency-induced hyperammonemia under conditions of well-controlled food intake. Rats were studied 1 and 2 weeks after surgery, since we previously showed that button-portacaval-shunted rats start to recover from several of the metabolic disturbances of portacaval shunting after 3-4 weeks, probably as a consequence of the development of hepatopetal shunts (26).

In the current experiments, the absence of differences in food intake and body weight between the PCSBDL, PCS-PF and PF group suggests that the differences between the PCS-PF group and the PF group are due to the effects of portacaval shunting. Similarly, the differences between the PCSBDL group and PCS-PF rats are related to the sequelae of aggravated parenchymal liver injury and cholestatic jaundice, induced by common bile duct ligation.

Arterial ammonia levels were comparably elevated in PCS-PF and PCSBDL rats, and this was reflected by a similar increment in muscle tissue ammonia. However, no net 
ammonia uptake was observed across the hindquarter during chronic liver insufficiency-induced hyperammonemia or in the PF group, contrasting with earlier reports on net muscle ammonia uptake in humans with chronic liver insufficiency $(1,3,11)$. Tracer studies have reported uni-directional skeletal muscle ammonia uptake in healthy humans (2), patients with chronic liver disease (2) and normal and $\mathrm{CCl}_{4}$ liver-injured rats (31). It must be noted, however, that uni-directional uptake measurements do not give adequate information on net fluxes $(2,32)$.

In healthy humans net muscle ammonia uptake was observed by Eriksson et al. (33), but not by Ganda and Ruderman (3) and Webster and Gabuzda (11). Since metabolic trapping of ${ }^{13} \mathrm{~N}$ ammonia was observed in healthy volunteers (2), despite absence of net ammonia uptake by muscle $(3,11)$, it has been concluded that muscle must continuously use and produce ammonia (2). Whether muscle ammonia uptake actually occurs in a specific situation may be related to (changes in) arterial ammonia levels. Thus, there is a good correlation between arterial ammonia levels and muscle ammonia uptake, and most studies reporting net ammonia uptake in humans were performed in hyperammonemic patients with decompensated cirrhosis (1,3,11). Also, it has been shown that in normal dogs $(12,34)$, rats $(14,13,35)$ and pigs $(36)$, ammonia infusion induced net skeletal muscle ammonia uptake. These acute experiments were performed in either briefly fasted $(12,13,34,36)$ or fed $(14,35)$, but otherwise healthy animals. Acute ammonia infusion in healthy animals artificially enhances partial ammonia pressure in arterial blood, creating an ammonia pressure gradient across cellular membranes, which forces ammonia into the cells (12). Such a situation does not fully resemble chronic hyperammonemia, in which a new equilibrium between arterial and tissue ammonia is reached. Therefore, we do not think that muscle contributes importantly to net ammonia detoxification at stable ammonia levels during chronic liver insufficiency-induced hyperammonemia. This does not, however, rule out a role for muscle in ammonia trapping during sudden elevations of arterial ammonia levels.

In PF as well as PCS-PF and PCSBDL rats, surgery and 7 days of semi-starvation resulted in an increase in arterial glutamine concentrations. However, no differences between the groups were observed on day 14, despite substantial differences in arterial
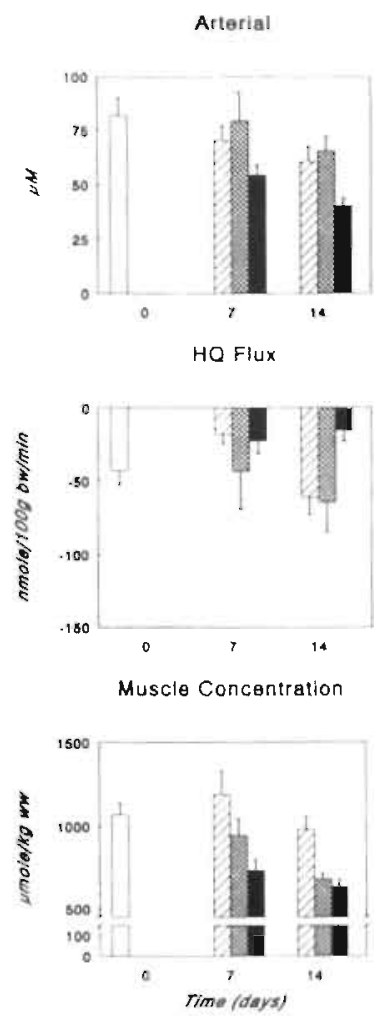

NOAM PCSBDL

Figure 4. Glutamate: arterial concentrations (upper panel; $\mu \mathrm{M}$ ), hindquarter fluxes (middle panel; nmole.100 $\mathrm{g}$ body $\left.\mathrm{wt}^{-1} \mathrm{~min}^{-1}\right)$ and gastrocnemius muscle tissue concentrations (lower panel; $\mu$ mole $\mathrm{kg}$ wel $\mathrm{w}^{-1}$ ) in normal control rats (NORM, open bars), sham operated pair-fed rats (PF, hatched bars), portacaval shunted rats (PCS-PF, cross hatched bars) and in rats with portacaval shunts and bile duct ligation (PCSBDL, filled bars). Data are means $\pm S E M ; N=6$ to 10 per group. 
ammonia concentrations. These findings suggest that the regulation of arterial glutamine levels is more related to nutritional factors than to hyperammonemia or liver insufficiency per se.

Hindquarter glutamine efflux was overall increased in both hyperammonemic groups compared with the $\mathrm{PF}$ group, a difference mainly caused by enhanced muscle glutamine release at day 7 . Simultaneously, muscle glutamine levels were similarly elevated at all time points in all operated groups compared with normal rats and no differences were observed between PF, PCS-PF and PCSBDL rats. This deserves some comments. First, the similar skeletal muscle glutamine levels despite remarkable differences in muscle ammonia levels between the PF and the chronic liver insufficiency-induced hyperammonemia groups suggests that tissue glutamine levels are related to nutritional factors, rather than to hyperammonemia or liver insufficiency. Second, the enhanced glutamine efflux in the chronic liver insufficiency groups compared to PF rats in spite of similar food intakes and body weights in these groups, suggests that the enhanced muscle glutamine release is related to liver insufficiency and/or hyperammonemia, rather than nutritional status. Finally, because both at day 7 and 14 muscle glutamine concentrations and efflux were increased in the hyperammonemic rats, it seems likely that muscle glutamine synthesis is enhanced in the PCS-PF and PCSBDL group.

Because enhanced glutamine production requires precursors for both the nitrogen moiety and the carbon skeleton, it is of particular interest to consider some of the metabolites intimately linked to glutamine synthesis, especially since no net ammonia uptake was observed. Enhanced muscle glutamate uptake could provide immediate precursors for glutamine synthesis. Interestingly, arterial glutamate concentration were similar in PF and PCS-PF rats, but decreased in the PCSBDL group. Also, the decreased muscle glutamate uptake in the PCSBDL rats could be causally related to the dramatic decrease in muscle glutamate in the PCSBDL group. However, these observations also could indicate that the changes in muscle glutamate levels are caused by enhanced glutamine synthesis consuming glutamate.

Additional indirect evidence for enhanced glutamate consumption in glutamine synthesis is provided by the decreased muscle BCAA concentrations, which may be explained by enhanced BCAA transamination to yield glutamate. The observed muscle

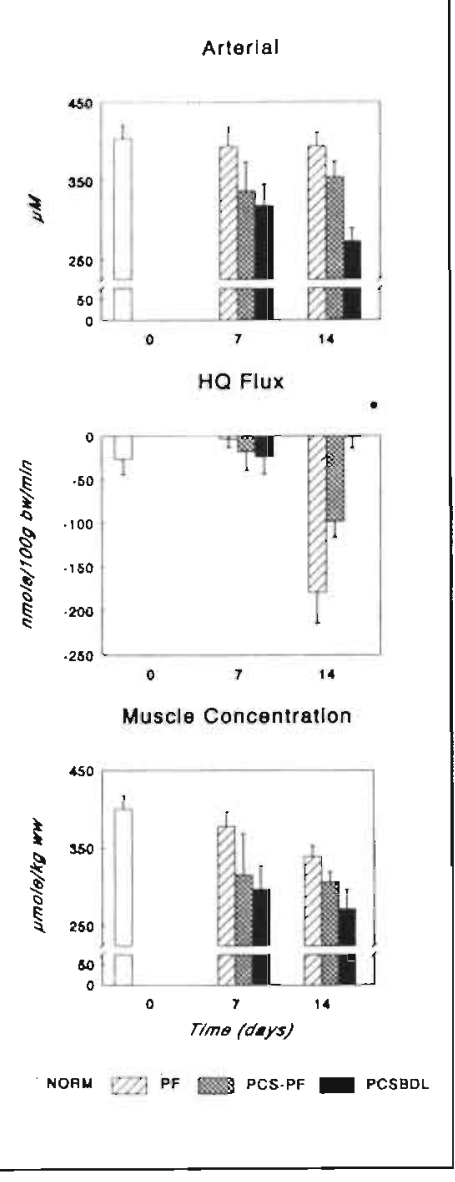

Figure 5. Branched-chain amino acids (BCAA): arterial concentrations (upper panel; $\mu \mathrm{M}$ ), hindquarter fluxes (middle panel; nmole $100 \mathrm{~g}$ body $\mathrm{w}^{-1} \mathrm{~min}^{-1}$ ) and gastrocnemius muscle tissue concentrations (lower panel; $\mu$ mole $\mathrm{kg}$ wet $\left.w^{-1}\right)$ in normal control rats (NORM, open bars), sham operated pairfed rats (PF, hatched bars), portacaval shunted rats (PCS-PF, cross hatched bars) and in rats with portacaval shunts and bile duct ligation (PCSBDL, filled bars). Data are means $\pm S E M ; ~ N=6$ to 10 per group. 
BCAA fluxes and arterial concentrations also seem compatible with this explanation. Similarly, the parallel changes in cellular levels of glutamate, aspartate and alanine in PCSBDL rats also suggest that glutamate levels are maintained in an equilibrium with these amino acids by aminotransferase activity in muscle (13).

The role of protein catabolism in providing glutamate (and possibly ammonia) for glutamine synthesis can be derived by considering muscle tissue phenylalanine concentration and flux (Figure 6), since this amino acid can be neither synthesized nor degraded in skeletal muscle $(32,37)$. Therefore, its net flux reflects the balance between protein synthesis and degradation (32). Thus, muscle phenylalanine concentration increased in PCSBDL rats compared to PCS-PF and PF rats, despite the fact that phenylalanine efflux was increased compared to PF rats, providing evidence for increased skeletal muscle protein breakdown. Like phenylalanine, the BCAA cannot be synthesized in skeletal muscle, but they can be degraded by oxidation and transamination $(32,37,38)$. Since the apparently enhanced protein breakdown in PCSBDL and PCS-PF rats did not augment skeletal muscle BCAA concentration, but instead was accompanied by decreased BCAA levels already at day 7 when fluxes were comparable, it seems justified to conclude that BCAA transamination to glutamate is enhanced in these rats. Therefore, it appears that the observed enhanced glutamine production is more related to accelerated protein catabolism and thus to malnutrition, than to enhanced ammonia detoxification. It seems self-evident to propose that in this situation the amide nitrogen of glutamine in not derived from bloodborne ammonia, but from amino acid catabolism. The mechanism underlying the enhanced net protein breakdown remains to be elucidated, but might be related to the elevated ammonia levels (39). Although ammonia is a weak base and therefore could theoretically be expected to raise the lysosomal $\mathrm{pH}$ and thereby inhibit protein degradation via the intra-lysosomal pathway, such an effect has never been demonstrated for ammonia (7). Also, the effect might be exerted via the extra-lysosomal pathway, because intra-lysosomal protein degradation only contributes $50 \%$ to muscle protein degradation under amino acid deprived conditions (for review, see 7). However, it cannot be excluded from these experiments that other factors (for review, see 7) play a role in this respect.

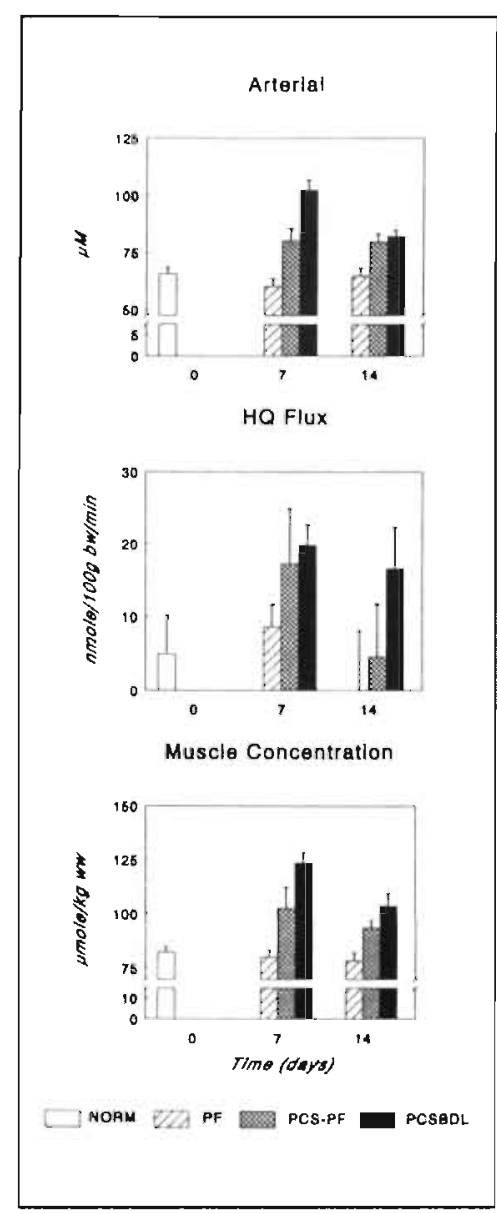

Figure 6. Phenylalanine: arterial concentrations (upper panel; $\mu \mathrm{M}$ ), hindquarter fluxes (middle panel; nmole.100 g body $\mathrm{wt}^{-1} \cdot \mathrm{min}^{-1}$ ) and gastrocnemius muscle tissue concentrations (lower panel; $\mu$ mole $\mathrm{kg}$ wet $\mathrm{wt}^{\prime \prime}$ ) in normal control rats (NORM, open bars), sham operated pair-fed rats (PF, hatched bars), portacaval shunted rats (PCS-PF, cross hatched bars) and in rats with portacaval shunts and bile duct ligation (PCSBDL, filled bars). Data are means $\pm S E M ; N=6$ to 10 per group 
In the present experiments, portacaval anastomosis combined with common bile duct ligation was introduced as another model to study nitrogen metabolism during chronic liver insufficiency. This model was chosen because it resembled theoretically more closely chronic liver insufficiency in humans than portacaval shunting alone. Thus, construction of a portacaval anastomosis alone induces, besides portasystemic shunting of gut-derived blood, a reduction of liver mass (absolute mass (40-43) as well as liver mass expressed as a percentage of body weight $(42,43)$ ). Urea synthesis capacity has been demonstrated to correlate strongly with hepatic mass under a variety of conditions, including portacaval shunting (41). In addition, urea synthesis capacity, has been shown to be limited in portacaval shunted rats, as evidenced by increased orotic aciduria (43). To these effects of portacaval anastomosis alone, the effects of chronic bile duct ligation were added.

Prolonged common bile duct ligation has been shown to induce periportal fibrosis, portal hypertension and portasystemic shunting (25) as well as metabolic dysfunction in the liver (38), also evidenced by increased plasma transaminase and decreased albumin levels $(24,25)$. The resulting jaundice is of a different etiology than that commonly encountered in cirrhotic patients, but nonetheless it is cholestatic like in patients with chronic hepatic insufficiency. It therefore can be expected to introduce in this model the impaired cellmediated immunity and Kuppfer cell dysfunction, as reported during prolonged biliary tree obstruction (23-25).

Because rats with portacaval shunting plus bile duct ligation showed subtle signs pointing to more pronounced hepatic encephalopathy, unrelated to differences in arterial levels of ammonia, other factors are probably also involved in the pathogenesis of hepatic encephalopathy. The present well-defined portacaval shunt/biliary obstruction model could be useful in future studies on the pathogenesis of chronic liver insufficiency-induced hyperammonemia and related encephalopathy.

In conclusion, the present experiments suggest that nutritional factors are causally related to many of the observations reported in chronic liver insufficiency studies, and this should be taken into account when interpreting results. Under conditions of standardized food intake in rats with chronic liver insufficiency-induced hyperammonemia, skeletal muscle glutamine levels appear to be maintained by enhanced amino acid catabolism and transamination to provide glutamate serving glutamine synthesis, possibly related to protein synthesis regulation (19). The unchanged arterial glutamine levels despite enhanced skeletal muscle glutamine efflux suggests that glutamine consumption under these conditions is enhanced in other organs. The fact that under these steady state conditions of hyperammonemia in overnight fasted animals, no ammonia uptake by the hindquarter was observed, does not rule out a role for skeletal muscle in ammonia trapping during rising ammonia concentrations. In that case, the ammonia gas pressure gradient across membranes may become enhanced, driving ammonia into the cell. Such a mechanism may be operative after a meal or after GI bleeding. Finally, portacaval shunting combined with common bile duct ligation appears to be a useful model to study nitrogen metabolism during chronic liver insufficiency-induced mild hyperammonemia in the rat. 


\section{REFERENCES}

1. Bessman SP, Bradley JE. Uptake of ammonia by muscle. Its implications in ammoniagenic coma. New Eng J Med 1955;253:1 143-1147.

2. Lockwood AH, McDonald JM, Reiman RE, Gelbard AS, Laughlin JS, Duffy TE. Plum F. The dynamics of ammonia metabolism in man. Effects of liver disease and hyperammonemia. J Clin Invest 1979;63:449-460.

3. Ganda OP, Ruderman NB. Muscle nitrogen metabolism in chronic hepatic insufficiency. Metabolism 1976;25:427-435.

4. Lund P. A radiochemical assay for glutamine synthetase, and activity of the enzyme in rat tissues. Bioch J 1970; 1 18:35-39.

5. Newsholme EA, Leech AR. Biochemistry for the Medical sciences. John Wiley \& Sons, New York, 1983.

6. Sherlock S. Diseases of the liver and biliary system. Blackwell Scientific Publications Oxford, 8th Edition, 1989.

7. Meijer AJ, Lamers WH, Chamuleau RAFM. Nitrogen metabolism and omithine cycle function. Physiol Rev 1990;70:701-748.

8. Zieve L. Pathogenesis of hepatic encephalopathy. Metab Brain Dis 1987;2:147-165.

9. Lin S, Raabe W. Ammonia intoxication:effects on cerebral cortex and spinal cord. J Neurochem 1985;44:1252-1258.

10. Record CO. Neurochemistry of hepatic encephalopathy. Gut 1991;32:1261-1263.

11. Webster LT, Gabuzda GJ. Ammonium uptake by the extremities and brain in hepatic coma. J Clin Invest 1958;37:414-424.

12. Hills AG, Reid EL, Kerr WD. Circulatory transport of L-glutamine in fasted mammals:cellular sources of urine ammonia. Am J Physiol 1967;223:1470-1476.

13. Ruderman NB, Lund P. Amino acid metabolism in skeletal muscle. Regulation of glutamine and alanine release in the perfused rat hindquarter. Isr J Med Sci 1972;8:295-302.

14. Rosado A, Flores G, Mora J, Sobéron G. Distribution of an ammonia load in the normal rat. Am J Physiol 1962;203:37-42.

15. Dejong CHC, Kampman MT, Deutz NEP, Soeters PB. Altered glutamine metabolism in rat portal drained viscera and hindquarter during hyperammonemia. Gastroenterology 1992;102:936-948.

16. Mullen KD, McCullough AJ. Problems with animal models of chronic liver disease:Suggestions for improvement in standardization. Hepatology 1989;9:500-505.

17. Dejong CHC, Deutz NEP. Soeters PB. Inter-organ nitrogen exchange during prolonged starvation in the rat. J Clin Nutr Gastroenterol 1991;6:176-183

18. Souba WW, Smith RJ, Wilmore DW. Glutamine metabolism by the intestinal tract. JPEN 1985;9:608-617.

19. Rennie MJ, Hundal HS, Babij P, MacLennan P. Taylor PM, Watt PW. Characteristics of a glutamine carrier in skeletal muscle have important consequences for nitrogen loss in injury, infection, and chronic disease. Lancet 1986;ii:1008-1011.

20. Ardawi MSM. Skeletal muscle glutamine production in thermally injured rats. Clin Sci 1988;74:165-172.

21. Ribeiro J, Nordlinger B, Ballet F, Coudray-Lucas C, Baudrimont M, Legendre C, Delelo R, Panis Y. Intrasplenic hepatocellular transplantation corrects hepatic encephalopathy in portacaval shunted rats. Hepatology 1992;15:12-18.

22. Maddison E, Dodd PR, Morrison M, Johnston GAR, Farrell GC. Plasma GABA, GABA-like activity and the brain GABA-benzodiazepine receptor complex in rats with chronic hepatic encephalopathy. Hepatology 1987;7:621-628.

23. Roughneen PT, Gouma DJ, Kulkami AD, Fanslow WF, Rowlands BJ. Impaired specific cellmediated immunity in experimental biliary obstruction and its reversibility by intemal biliary drainage. J Surg Res 1986;41:113-125.

24. Greve JW, Gouma DJ, Soeters PB, Buurman WA. Suppression of cellular immunity in obstructive jaundice is caused by endotoxins. A study in germ-free rats. Gastroenterology 1990;98:478-85.

25. Dunn CW, Horton JW, Megison SM, Vuitch MF. Contribution of portal systemic shunt to Kupffer cell dysfunction in obstructive jaundice. J Surg Res 1991;50:234-239.

26. de Boer JEG, Oostenbroek RJ, van Dongen JJ, Janssen MA, Soeters PB. Sequential metabolic characteristics following portacaval shunts in rats. Eur Surg Res 1986; 18:96-106. 
27. Deutz NEP, Dejong CHC, Athanasas G, Soeters PB. Partial enterectomy in the rat does not diminish muscle glutamine production. Metabolism 1992; in press.

28. Dejong CHC, Kampman MT, Deutz NEP, Soeters PB. Cerebral cortex ammonia and glutamine metabolism during liver insufficiency-induced hyperammonemia in the rat. $\mathrm{J}$ Neurochem 1992;59:1071-1079.

29. Dejong CHC. Deutz NEP, Soeters PB. A simple new method for repeated in vivo cerebral cortex flux measurement in rats. Lab Anim Sci 1992;42:280-285.

30. van Eijk HMH, van der Heijden MAH, van Berlo CLH, Soeters PB. Fully automated liquidchromatographic determination of amino acids. Clin Chem 1988;34:2510-2513.

31. Okita $\mathrm{M}$, Watanabe A, Tsuij T. Effect of branched chain amino acid on ${ }^{15} \mathrm{~N}$ incorporation into liver and skeletal muscle proteins following ${ }^{15} \mathrm{~N}$ ammonium chloride administration to carbon tetrachloride intoxicated rats. J Nutr Sci Vitaminol 1988;34:85-96.

32. Barten EJ, Revkin JH, Young LH, Zaret BL, Jacob R, Gelfand RA. An isotopic method for measurement of muscle protein synthesis and degradation in vivo. Bioch J 1987;245:223-228.

33. Eriksson LS, Broberg S, Bjorkman O, Wahren J. Ammonia metabolism during exercise in man. Clin Physiol 1985;5:325-336.

34. Fine A. The effects of ammonia infusion on ammonia and glutamine metabolism by liver and muscle in the normal dog. Contrib Nephrol 1985;47:1-8.

35. Chabrier G, Schlienger JL, Imler M. Etude du métabolisme musculaire de l'ammoniaque sur le train postérieur du rat intact. CR Soc Biol 1982;176:716-722.

36. Deutz NEP, Dejong CHC, Reijven PLM, Soeters PB. In vivo ammonia and glutamine flux measurements during hyperammonemia in rats and pigs. In: Bengtson $F$, Almdal $T$, Jeppsson $B$, Vilstrup H, eds. Progress in Hepatic Encephalopathy and Metabolic Nitrogen Exchange. CRC Press, Boca Raton, 1991;329-339.

37. Chang TW, Goldberg AL. The metabolic fates of amino acids and the formation of glutamine in skeletal muscle. J Biol Chem 1978;253:3685-3695.

38. Stames FH, Conti PS, Warren RS, Jeevanandam M, Brennan MF. Altered peripheral amino acid uptake in obstructive jaundice. J Surg Res 1987;42:383-393.

39. Wasterlain CG, Lockwood AH, Conn M. Chronic inhibition of brain protein synthesis after portacaval shunting. Neurology 1978;28:233-238.

40. Dunlop DS, Kaufman H, Zanchin G, Lajtha A. Protein synthesis rates in rats with portacaval shunts. J Neurochem 1984;43:1487-1489.

41. Brewer TG, Berry WR, Hamon JW, Walker SH, Dunn MA. Urea synthesis after protein feeding reflects hepatic mass in rats. Hepatology 1984;4:905-911.

42. Benjamin IS, Engelbrecht GHC, Saunders SJ, van Hoom Hickman R. Amino acid imbalance following portal diversion in the rat. The relevance of nutrition and of hepatic function. J Hepatol 1988;7:208-2|4.

43. Steele RD. Hyperammonemia and orotic aciduria in portacaval shunted rats. I Nutr $1984 ; 114: 210-216$. 


\title{
Gut during chronic liver failure ${ }^{1}$
}

To be published as: C.H.C. Dejong, N.E.P. Deutz, P.B. Soeters. Intestinal glutamine and ammonia metabolism during chronic liver insufficiency-induced hyperammonemia. Gut, accepted for publication.

\begin{abstract}
During liver insufficiency, besides portasystemic shunting, high arterial glutamine levels could enhance intestinal glutamine consumption and ammonia generation, thereby aggravating hyperammonemia. To investigate this hypothesis, portal drained viscera ( $\approx$ intestines) fluxes and jejunal tissue concentrations of ammonia and glutamine were measured in portacaval shunted/bile duct ligated, portacaval shunted and sham operated rats, 7 and 14 days after surgery, and in normal unoperated controls. Effects of differences in food intake were minimized by pair-feeding portacaval shunted and sham operated with portacaval shunted/biliary obstructed rats. At both time points, arterial ammonia was elevated in the liver insufficiency groups. Also, arterial glutamine was elevated in all operated groups compared with normal unoperated controls. At both time points, portal drained viscera ammonia production was reduced in portacaval shunted/biliary obstructed, portacaval shunted and sham operated rats compared with normal unoperated controls, and between these operated groups no major differences were observed. At day 7 in all operated groups portal drained viscera glutamine uptake was lower than in normal unoperated controls, but no major differences were observed at day 14 . These experiments demonstrate that portal drained viscera ammonia generation remains unchanged in rats with chronic liver insufficiency despite alterations in arterial glutamine concentrations and intestinal glutamine uptake. The hyperammonemia seems to be mainly determined by the portasystemic shunting.
\end{abstract}

\footnotetext{
'This chapter is an exact copy of the original submission and consequently does not present all data of the experiments. Additional data are available on request. The data described in this chapter were obtained in the same series of experiments as those described in chapter 8; for readability the data concerning muscle and gut metabolism have been described in separate chapters (8\&9).
} 


\section{INTRODUCTION}

Ammonia is still considered to be of crucial importance in the pathogenesis of hepatic encephalopathy (1). Gut and kidney are generally considered to be the most important sites of ammonia production (2-5). In the gut, the main sources of ammonia production are the bacterial breakdown of urea $(2,3,6,7)$ and the mucosal utilization of glutamine as an energy substrate $(2,3,7,8)$. In the physiological situation virtually all ammonia generated in the gut is immediately cleared by hepatic urea synthesis, and thus hepatic venous ammonia levels are lower than arterial concentrations. Therefore, in healthy individuals, systemic ammonia levels are probably mainly set by the interaction between renal ammonia production and ammonia consumption by other organs. During chronic liver disease this situation changes drastically, since ammonia generated in the intestines by-passes hepatic clearance by intraand/or extra-hepatic portasystemic shunts $(9,10)$. Also, the diminished urea synthesis and glutamine synthetase capacity (9-11) may reduce ammonia detoxification in the liver. These combined factors probably cause the systemic hyperammonemia during liver insufficiency.

Systemic hyperammonemia during chronic liver failure has been suggested to lead to enhanced ammonia detoxification via alternative pathways (12). It is generally believed that the energy-dependent synthesis of glutamine from equimolar amounts of glutamate and ammonia is the most important of these pathways (13). This glutamine synthesis in glutamine synthetase containing organs, presumably mainly skeletal muscle and brain (14), probably contributes to the commonly observed elevated arterial glutamine levels during liver insufficiency-induced hyperammonemia (12). Because intestinal glutamine uptake is concentration-dependent (7), these elevated arterial glutamine levels in turn could increase intestinal glutamine utilization and subsequent ammonia liberation.

A considerable amount of research has been performed concerning several aspects of ammonia and glutamine metabolism during acute and chronic liver insufficiency (see e.g. (4-6,12,15-18)), also in our laboratory $(19,20)$. However, despite the well-known effects of food intake on nitrogen metabolism in general and more specifically on glutamine and ammonia metabolism (21), very little studies have been performed under conditions of controlled food intake during liver insufficiency-induced hyperammonemia (17).

The hypothesis underlying the present study was that during chronic liver insufficiency, besides portasystemic shunting, high arterial glutamine levels could aggravate hyperammonemia by enhanced intestinal glutamine consumption and ammonia generation. To test this hypothesis the exchange of ammonia, glutamine, and several other amino acids across the portal drained viscera ( $\approx$ gut) as well as jejunal tissue concentrations were measured in two models of chronic liver insufficiency-induced hyperammonemia in the rat: portacaval shunting and portacaval shunting combined with bile duct ligation, as well as in appropriate controls. Portacaval shunting combined with biliary tract ligation was recently suggested to be a reliable, new model for chronic liver insufficiency-induced hyperammonemia and to induce a more pronounced clinical degree of hepatic encephalopathy than portacaval shunting alone (22). To minimize effects of differences in food intake, the portacaval shunted and the sham operated groups were pair-fed with the anorectic portacaval shunt/biliary obstruction group. 
Animals Male Wistar rats $(\mathrm{n}=56,300 \pm 25 \mathrm{~g})$ were used throughout. Rats were housed under standard conditions (12/12 h light/dark cycle) and received standard food and water ad libitum until surgery. Rats were maintained and humanely cared for according to the recommendations of the Guide for the Care and Use of Laboratory Animals, as applied in our institute.

Groups Four groups were studied: 1 PCSBDL group. Portacaval shunting (PCS) combined with bile duct ligation (BDL) in one surgical session. 2 PCS-PF group. Portacaval shunting. $\underline{3} P F$ group. Laparotomy and manipulations as in PCS-PF rats, but without shunting (sham operation). 4 NORM group. Sampling without previous surgery; normal unoperated control rats, receiving food ad libitum.

Before surgery, rats were randomly assigned to one of the groups. Surgical procedures were of equal duration in PCS-PF and PF rats and their individual PCSBDL mate. After resuscitation from surgery, PCSBDL, PCS-PF and PF rats were placed in metabolic cages. PCSBDL rats had free access to standard pellet food. In these rats, daily food intake was recorded and this amount was administered to fixed PCS-PF and PF mates to minimize effects of differences in food intake (pair-feeding: PF). In the PCSBDL, PCS-PF and PF groups blood was sampled 7 or 14 days after surgery (see below). Body weight was measured at the time intervals indicated in the figures. Water was administered ad libitum in all groups.

Behavior Before entering the experiments and before sampling, behavior of all rats was studied during a 5 min period to estimate the degree of hepatic encephalopathy. Specific attention was paid to appearance, presence of lethargy, spontaneous locomotor activity and exploratory behavior, presence of tiptoe gait, toeing out and arched back, reaction to tail pinching and presence of ataxia. Since the PCSBDL rats were jaundiced, this staging procedure could not be performed blinded.

Surgery Surgery and sampling were performed under ether anesthesia. Portacaval shunting was performed by the button technique $(19,23)$. Biliary obstruction was achieved by ligating the common bile duct near the liver and near the duodenum and removing the piece between the ligatures. In PCS-PF and PF rats, the bile duct was manipulated, but not dissected, to avoid stenosis.

Sampling Prior to sampling, rats were fasted overnight. Sampling procedures were performed as described elsewhere $(19,21,24)$. Briefly, rats were anesthetized $30 \mathrm{~min}$ before sampling and the portal vein and a tertiary branch of the superior mesenteric vein were cannulated with needle bearing micro-catheters, fixed in place with cyanoacrylate adhesive. The right common carotid artery was catheterized with polyethylene tubing (PE 50). For flow determinations, after a priming dose, a $5 \mathrm{mM}$ iso-osmolar, $\mathrm{pH}$ adjusted para-aminohippuric acid $(\mathrm{PAH})$ solution was continuously infused in the mesenteric vein catheter $\left(25 \mu \mathrm{L} \cdot \mathrm{min}^{-1}\right)$, allowing for a $20 \mathrm{~min}$ period to attain steady state concentrations $(20,25)$. Blood was slowly and simultaneously aspirated from the portal vein and carotid artery and put in heparinized tubes on ice. Finally, approximately $10 \mathrm{~cm}$ distal to Treitz's ligament, a $10 \mathrm{~cm}$ piece of jejunum was excised and frozen in liquid nitrogen (24). Rats were killed by an overdose of the anaesthetic. These procedures permit simultaneous determination of blood flow and arteriovenous concentration difference (=flux) across the portal drained 
Table 1. Behavior.

\begin{tabular}{|c|c|c|c|c|c|c|c|c|}
\hline & \multirow{2}{*}{$\begin{array}{l}\text { DAY O } \\
\text { NORM }\end{array}$} & \multicolumn{3}{|c|}{ DAY 7} & \multicolumn{4}{|c|}{ DAY 14} \\
\hline & & $\mathrm{PF}$ & PCS-PF & PCSBDL & $\mathrm{PF}$ & PCS-PF & PCSBDL & \\
\hline 'Gerbil' appearance & $0 / 10$ & $4 / 8$ & $5 / 8$ & $8 / 8 \quad 9$ & $1 / 8$ & $5 / 8$ & $6 / 6$ & . \\
\hline Toeing out (hindlimbs) & $0 / 10$ & $0 / 8$ & $0 / 8$ & $6 / 8 \quad \pi$ & $0 / 8$ & $6 / 8$ & $6 / 6$ & s \\
\hline Tiptoe gait & $0 / 10$ & $0 / 8$ & $1 / 8$ & $3 / 8$ & $0 / 8$ & $1 / 8$ & $6 / 6$ & su \\
\hline 'Duck' gail & $0 / 10$ & $0 / 8$ & $1 / 8$ & $3 / 8$ & $0 / 8$ & $2 / 8$ & $6 / 6$ & su \\
\hline Arched back & $0 / 10$ & $0 / 8$ & $1 / 8$ & $3 / 8$ & $0 / 8$ & $3 / 8$ & $5 / 6$ & 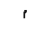 \\
\hline Ataxia & $0 / 10$ & $0 / 8$ & $0 / 8$ & $2 / 8$ & $2 / 8$ & $6 / 8$ & $6 / 6$ & $L^{\prime}$ \\
\hline
\end{tabular}

All items were scored on a dichotomic basis i.e. present or absent. $X N$ in the table indicates that the item was $X$ times present

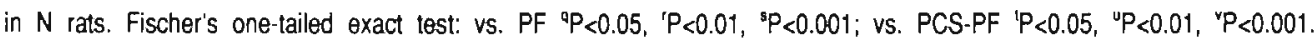

Tabłe 2. Arterial concentrations.

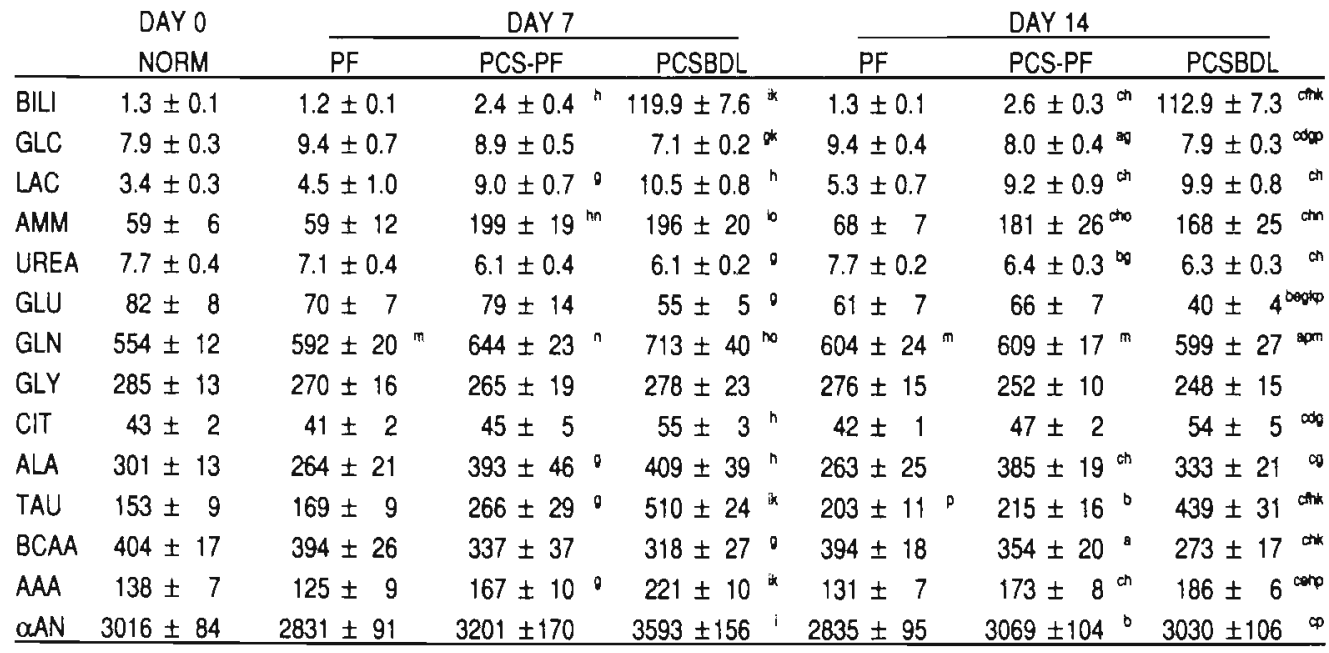

Data are expressed as means \pm SEM in $\mu \mathrm{M}$, except urea, glucose (GLC) and lactate (LAC) in mM. BCAA (branched-chain amino acids LEU, ILE and VAL); AAA (aromatic amino acids TYR + PHE). Symbols: ANOVA for group effects; $t=7$ to $t=14$ days: vs. $P F{ }^{A} P<0.05,{ }^{D} P<0.01,{ }^{C} P<0.001$; vs. $P C S-P F{ }^{d} P<0.05, " P<0.01,{ }^{P} P<0.001$. Mann-Whitney $U$ for differences between

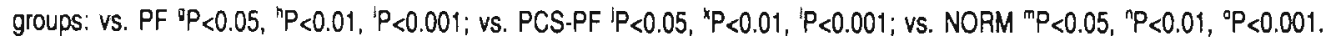
Wilcoxon test: "not significanlly different from zero. ONEWAY procedure for time effects within groups ${ }^{\circ}<<0.05$.

viscera ( $\approx$ intestines) $(19,21,24)$, and tissue concentration measurements.

Analysis Blood samples were kept on ice during processing. Plasma for amino acids, ammonia and urea was obtained by whole centrifugation. Centrifugations were performed at $4{ }^{\circ} \mathrm{C}$ during $5 \mathrm{~min}$ at $8,800 \mathrm{~g}$. Hematocrit was obtained with a microfuge. Plasma and tissue was stored at $-70^{\circ} \mathrm{C}$. For tissue ammonia and amino acid determinations, $5 \%(\mathrm{w} / \mathrm{v})$ sulfosalicylic acid extracts were prepared (19). Tissue water content was determined as described 
Table 3. Portal drained viscera metabolite fluxes and plasma flows.

\begin{tabular}{|c|c|c|c|c|c|c|c|}
\hline & \multirow{2}{*}{$\begin{array}{l}\text { DAY 0 } \\
\text { NORM }\end{array}$} & \multicolumn{3}{|c|}{ DAY 7} & \multicolumn{3}{|c|}{ DAY 14} \\
\hline & & $\mathrm{PF}$ & PCS-PF & PCSBDL & $\mathrm{PF}$ & PCS-PF & CSBDL \\
\hline .ow & $3.1 \pm 0.9$ & $0.8 \pm 0.2^{n}$ & $2.3 \pm 1.4$ & $1.7 \pm 0.9$ & $2.4 \pm 1.2$ & $2.7 \pm 1.1$ & 1.0 \\
\hline AMM & $413 \pm 62$ & $99 \pm 33^{n}$ & $221 \pm 47^{\mathrm{m}}$ & $160 \pm 37^{n}$ & $295 \pm 74^{\circ}$ & $328 \pm 81$ & $260 \pm 140$ \\
\hline GLU & $20 \pm 14$ & $5 \pm 1$ & $-1 \pm 15^{*}$ & $13 \pm 3$ & $40 \pm 9^{p}$ & $16 \pm 7^{*}$ & $34 \pm 8$ \\
\hline GLN & $-486 \pm 49$ & $-127 \pm 12^{\circ}$ & $-351 \pm 48$ & $-312 \pm 19 m$ & $-566 \pm 53^{p}$ & $-386 \pm 64$ & $-308 \pm 31 \mathrm{~km}$ \\
\hline GLY & $47 \pm 19$ & $19 \pm 8$ & $33 \pm 19$ & $3 \pm 7^{*}$ & $118 \pm 17^{p}$ & $57 \pm 12^{\circ}$ & $4 \pm 20$ \\
\hline $\mathrm{CIT}$ & $51 \pm 9$ & $10 \pm 2^{n}$ & $45 \pm 14$ & $31 \pm 4^{n}$ & $67 \pm 10^{p}$ & $57 \pm 12$ & 14 \\
\hline$A L A$ & $169 \pm 57$ & $74 \pm 20$ & $215 \pm 69$ & $123 \pm 14$ & $403 \pm 45^{p}$ & $256 \pm 57^{\circ}$ & $105 \pm 52$ \\
\hline$A U$ & $30 \pm 15$ & $11 \pm 9^{*}$ & $9 \pm 30$ & $-28 \pm 6$ & $83 \pm 31^{\circ}$ & $72 \pm 35$ & $-50 \pm 27^{\mathrm{math}}$ \\
\hline BCAA & $-26 \pm 31 *$ & $14 \pm 9^{*}$ & $27 \pm 27$ * & $-6 \pm 4^{\circ}$ & $75 \pm 24^{P}$ & $44 \pm 23$ * & $-11 \pm 12$ \\
\hline AAA & $-11 \pm 15 *$ & $2 \pm 3$ * & $5 \pm 9^{\prime \prime}$ & $-4 \pm 5$ & $19 \pm 6^{p}$ & $8 \pm 5$ & $-19 \pm 7$ \\
\hline$\alpha \mathrm{AN}$ & $-315 \pm 180$ * & $16 \pm 46^{\circ}$ & $-24 \pm 174$ * & $-229 \pm 35^{n}$ & $399 \pm 101^{\circ}$ & $186 \pm 126$ & $-285 \pm 159$ \\
\hline$\underline{F E}_{\text {OW }}$ & $28.1 \pm 2.5$ & $27.9 \pm 2.5$ & $23.7 \pm 3.7^{n}$ & $26.3 \pm 1.9$ & $39.5 \pm 3.1^{\mathrm{mp}}$ & $23.6 \pm 3.9^{\text {on }}$ & $22.9 \pm 2.8$ \\
\hline
\end{tabular}

Data are means \pm SEM in nmole $100 \mathrm{~g}$ body $\mathrm{wt}^{-1} \mathrm{~min}^{-1}$, except plasma flows in $\mathrm{mL} \cdot 100 \mathrm{~g}$ body $\mathrm{wt}^{-1} \cdot \mathrm{min}^{-1}$. For significance symbols, see Table 2 .

Table 4. Jejunal concentrations and water content.

\begin{tabular}{|c|c|c|c|c|c|c|c|c|c|}
\hline & 0 & & ד & & & & DAY 14 & & \\
\hline & NORM & PF & PCS.PF & PCS & & PF & PCS-PF & PCSBDL & \\
\hline $\mathrm{H}_{2} \mathrm{O}$ & $76.9 \pm 0.6$ & $77.5 \pm 0.7$ & $77.6 \pm 0.6$ & $76.9 \pm 0.4$ & & $77.3 \pm 0.5$ & $78.0 \pm 0.9$ & $77.1 \pm 0.6$ & \\
\hline MM & $1436 \pm 83$ & $1213 \pm 69$ & $1688 \pm 146$ & h $\quad 1681 \pm 153$ & 8 & $1391 \pm 91$ & $1541 \pm 83^{\circ}$ & $1634 \pm 152$ & \\
\hline$S P$ & $752 \pm 47$ & $950 \pm 50^{n}$ & $853 \pm 60$ & $862 \pm 42$ & & $865 \pm 38$ & $749 \pm 52$ s & 53 & \\
\hline$U$ & $2579 \pm 74$ & $2628 \pm 75$ & $2872 \pm 146$ & $2853 \pm 96$ & & $2683 \pm 61$ & $2533 \pm 145$ & 2839 & \\
\hline$L N$ & $693 \pm 46$ & $778 \pm 34$ & $827 \pm 16$ & $870 \pm 35$ & & $693 \pm 25$ & $740 \pm 48$ & & \\
\hline$Y$ & $1112 \pm 46$ & $1071 \pm 62$ & $1171 \pm 164$ & $958 \pm 70$ & & $1220 \pm 67$ & $1035 \pm 45^{\circ}$ & 40 & \\
\hline & $171 \pm 10$ & $177 \pm 6$ & $192 \pm 20$ & & & $190 \pm 9$ & $185 \pm 12$ & & \\
\hline A & $1041 \pm 52$ & $1022 \pm 75$ & $1180 \pm 96$ & $1171 \pm 76$ & & $1268 \pm 93$ & D $\quad 1147 \pm 51$ & & \\
\hline $\mathrm{AU}$ & $14821 \pm 645$ & $15666 \pm 745$ & $17105 \pm 661$ & $17641 \pm 518$ & & $17142 \pm 469$ & $16957 \pm 802$ & $18292 \pm 339$ & \\
\hline CAA & $510 \pm 24$ & $506 \pm 44$ & $542 \pm 58$ & $348 \pm 25$ & & $682 \pm 73$ & $534 \pm 78$ & $314 \pm 9$ & \\
\hline AA & $219 \pm 25$ & $198 \pm 21$ & $300 \pm 38$ & $272 \pm 19$ & & $262 \pm 27$ & $287 \pm 33$ & $234 \pm 14$ & \\
\hline$\triangle A N$ & $23404 \pm 773$ & $24311 \pm 663$ & $26662 \pm 771$ & $\therefore \quad 26551 \pm 480$ & 8 & $26657 \pm 614$ & $P \quad 25655 \pm 834$ & $26777 \pm 519$ & \\
\hline
\end{tabular}

Data are expressed as mean \pm SEM in $\mu$ mole $\mathrm{kg}$ wet weight ${ }^{-1}(\mathrm{wW})$. For significance symbols, see Table 2.

previously (20). Ammonia, urea, lactate and glucose were determined enzymatically (19), amino acids by HPLC technique (26). PAH was determined as described previously (19). Total bilirubin was determined in plasma using a commercial kit (Roche, Basel, Switzerland). The coefficient of variance for all determinations was below $4 \%(19,26)$.

Calculations Plasma PAH concentration was calculated from whole blood PAH concentrations using the hematocrit. Plasma flow was calculated using the formula $\mathrm{F}_{\mathrm{PDV}}=\mathrm{L} /\left([\mathrm{PAH}]_{\mathrm{P}}-[\mathrm{PAH}]_{\mathrm{A}}\right)$. In this formula, $\mathrm{F}_{\mathrm{PDV}}$ is the portal drained viscera plasma flow 
$\left(\mathrm{mL} \min ^{-1}\right), \mathrm{I}$ is the infused PAH $\left(\mu\right.$ mole $\left.\min ^{-1}\right)$, and $[\mathrm{PAH}]_{P, \mathrm{~A}}$ is the plasma PAH concentration $(\mu \mathrm{M})$ in the portal vein and carotid artery, respectively. Portal drained viscera fluxes (nmole $100 \mathrm{~g}$ body weight ${ }^{1} \mathrm{~min}^{-1}$ ) were calculated as plasma flow times portal venous-arterial concentration difference. Positive figures mean net efflux, negative values reflect net uptake. Fractional glutamine extraction was calculated as the arterial-portal vein concentration difference divided by the arterial concentration $(((\mathrm{A}-\mathrm{PV}) / \mathrm{A}) \cdot 100 \%)$. Urea values were corrected for ammonia. $\alpha$-Amino nitrogen $(\alpha A N)$ was calculated as the sum of individual amino acids measured (26).

Statistics (SPSS/PC+ Statistical Software Package, version 3.1 (SPSS Inc, Chicago, IL, USA)). Data presented are means $\pm S E M, P<0.05$ was considered significant. The following tests were used: analysis of variance for group effects (ANOVA) and time effects within groups (ONEWAY); the Mann-Whitney $U$ non-parametric test for differences between groups at specific time points; Wilcoxon's nonparametric test for differences from zero; Fischer's exact test for differences in encephalopathy stages (one-tailed, binomial testing). Significances are indicated in Tables 1-4.

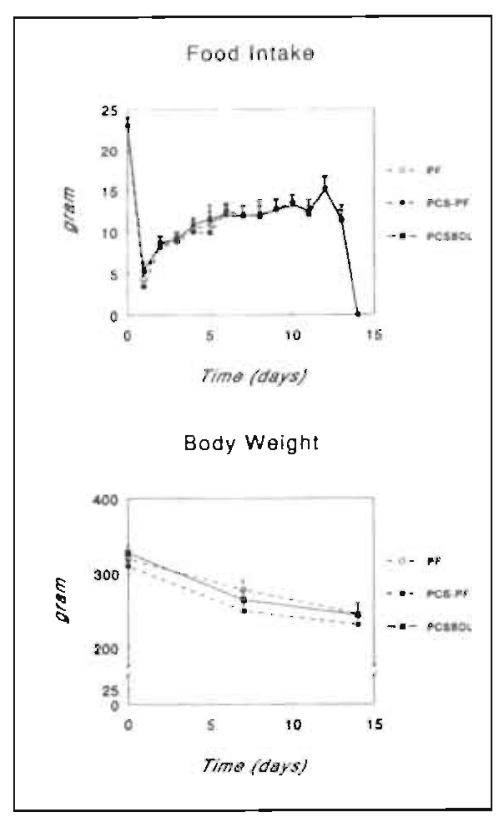

Figure 1. Food intake (upper panel) and body weight (lower panel) in PCSBDL (filled squares), PCS-PF (filled circles) and PF (open circles) rats during 14 days of pairfeeding. The plotted individual curves are means \pm SEM of 14 to 16 rats in the first 7 days and 6 to 8 rats between day 7 and 14 .

\section{RESULTS}

General Food intake and body weight (Figure 1) were similar in PCSBDL, PCS-PF and PF rats. Specifically in PCSBDL rats, no signs of systemic or local inflammation or impaired wound healing were observed. However, these rats appeared to be more encephalopathic, as evidenced by abnormalities in gait, decreased spontaneous locomotor activity and exploratory behavior (Table 1). Total bilirubin levels (Table 2) were increased in PCS-PF compared with PF, as observed previously (27), and in PCSBDL compared with PCS-PF rats. In both hyperammonemic groups, glucose levels were decreased and lactate levels were elevated in comparison to PF rats (Table 2). Portal drained viscera plasma flow (Table 3 ) was decreased in the PF group at day 7 when compared with normal unoperated control rats, but no further differences in portal plasma flow were observed between the PCSBDL, PCS-PF and PF groups.

Ammonia (Figure 2; Table 2-4) Arterial ammonia levels were equally elevated in PCSBDL and PCS-PF rats at both day 7 and 14. No major differences in portal drained viscera ammonia production were observed between PCSBDL, PCS-PF and PF rats, and portal drained viscera ammonia production in the hyperammonemic groups never exceeded that in normal control rats. In fact, it was lower in all operated groups at day 7 compared with normal unoperated controls (NORM). Although no major changes occurred in portal 
drained viscera ammonia release, jejunal tissue ammonia concentrations were elevated in both hyperammonemic groups.

Glutamine (Figure 3; Table 2-4) Arterial glutamine concentrations were always higher in PCSBDL, PCS-PF and PF than in NORM rats. Also, at day 7 arterial glutamine levels were elevated in PCSBDL compared to $P F$ rats, but no differences were observed at day 14. At day 7, portal drained viscera glutamine uptake was enhanced in both hyperammonemic groups compared to sham operated animals, mainly caused by a reduced portal vein plasma flow in the PF group. However, no major differences were observed at day 14 . Although arterial glutamine levels were always higher in the hyperammonemic groups, portal drained viscera glutamine uptake never exceeded that in normal controls. Simultaneously, jejunal glutamine levels were elevated in PCSBDL compared with PF rats at both time points. The observed changes in glutamine and ammonia handling make it of special interest to look at some key metabolites intimately related to the intestinal breakdown of glutamine: alanine, glutamate and citrulline.

Alanine (Figure 4; Table 2-4) Arterial alanine concentrations were elevated in both chronic liver insufficiency groups at both time points. Portal drained viscera alanine efflux was overall decreased in the PCSBDL group compared with both PF and PCS-PF rats (ANOVA day 7 to 14). In addition, at day 14 alanine release was lower in the PCS-PF group compared with PF rats. Despite these changes, no major differences in tissue alanine were observed.

Glutamate (Figure 5; Table 2-4) At both time points, in PCSBDL rats arterial glutamate was diminished compared with PCS-PF and PF rats. Only minor amounts of glutamine uptake could be accounted for by glutamate release, in agreement with the literature $(7,28)$. Glutamate release by the portal drained viscera was slightly lower in the PCS-PF group compared with all other groups, but no further differences were observed. Interestingly, jejunal tissue glutamate levels were increased in the PCSBDL compared with the PF group, despite decreased arterial glutamate levels.

Citrulline (Table 2-4) Arterial citrulline concentrations were elevated in the PCSBDL group compared to PCS-PF and PF rats. Citrulline production by the gut did not show major
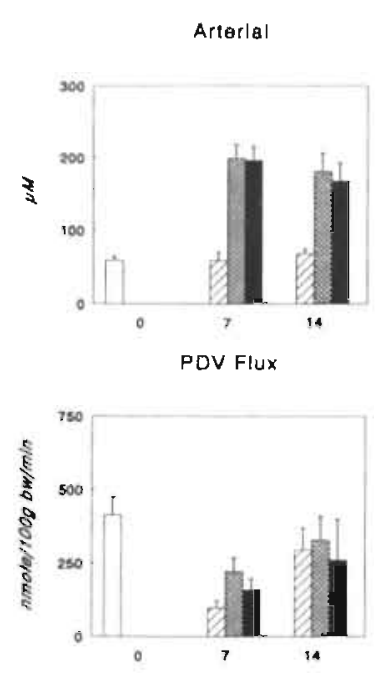

Gut Concentration

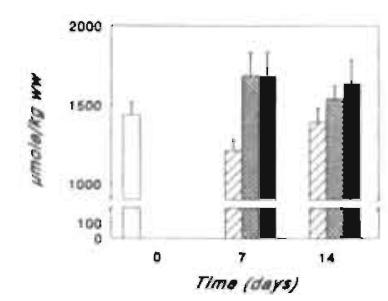

NOAM QZZ PF

PCseol

Figure 2. Ammonia: arterial concentrations (upper panel; $\mu \mathrm{M}$ ), portal drained viscera fluxes (middle panel; nmole.100 g body $w^{-1} \min ^{-1}$ ) and jejunal tissue concentrations (lower panel; $\mu$ mole kg wet wt') in normal control rats (NORM, open bars), sham operated pair-fed rats (PF, hatched bars), portacaval shunted rats (PCS-PF, cross hatched bars) and in rats with portacaval shunts and bile duct ligation (PCSBDL, filled bars). Data are means $\pm S E M ; N=6$ to 10 per group. 
differences between the groups, although tissue citrulline levels were elevated in the PCSBDL group.

Remaining amino acids - important observations (Table 2-4) Arterial BCAA levels were decreased in both chronic liver insufficiency groups compared to $\mathrm{PF}$ at both time points. Interestingly, although BCAA fluxes were not significantly different from zero in most groups, jejunal tissue BCAA levels were decreased in PCSBDL rats compared with all other groups. Arterial taurine levels were elevated in PCS-PF compared with PF rats and in PCSBDL compared with all other groups. Taurine release by the portal drained viscera in the PCS$\mathrm{PF}$ and PF group reversed to uptake in the PCSBDL group, although jejunal tissue taurine levels were already elevated.

\section{DISCUSSION}

The present experiments were designed to study intestinal ammonia and amino acid metabolism during chronic liver insufficiency-induced hyperammonemia. Specifically, we were interested whether elevated arterial glutamine levels during chronic liver insufficiency would lead to enhanced intestinal glutamine uptake and subsequent ammonia release, thereby aggravating the already existing portasystemic shunting-induced hyperammonemia. To minimize the influence of differences in nutritional status, rats were studied under conditions of well-controlled food intake. Studies were performed 1 and 2 weeks after surgery, since we previously showed that button-portacaval-shunted rats recover from several of the metabolic disturbances of portacaval shunting after 3-4 weeks, probably due to the development of hepatopetal shunts (23).

In the current experiments, the similar food intake and body weight in PCSBDL, PCS-PF and PF rats suggests that differences between PCS-PF and PF rats are due to portacaval shunting. Similarly, differences between PCSBDL and PCS-PF rats are related to the aggravated hepatic parenchymal injury and cholestatic jaundice, induced by prolonged biliary obstruction (29-32).

Arterial ammonia levels were equally elevated in PCS-PF and PCSBDL rats, compared with both PF and normal control rats. This was observed at day 14, when intestinal ammonia production was similar in NORM, PF, PCS-PF and PCSBDL rats, as well as at day 7, when ammonia liberation was decreased in all operated groups compared to NORM rats. The
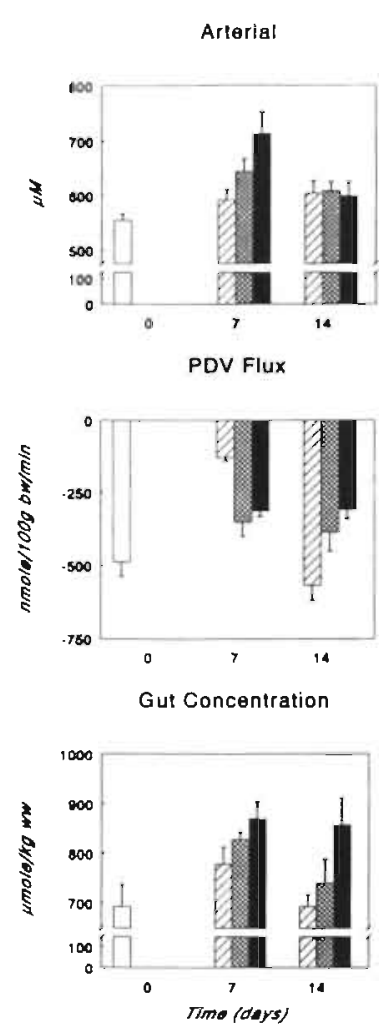

$\square$ NORM ZOA PF 2 PCS.PF $\square$ PCSEDL

Figure 3. Glutamine: arterial concentrations (upper panel; $\mu \mathrm{M}$ ), portal drained viscera fluxes (middle panel; nmole-100 g body $\left.w t^{-1} \min ^{-1}\right)$ and jejunal tissue concentrations (lower panel; $\mu$ mole $k g$ wet $w^{-1}$ ) in normal control rats (NORM, open bars), sham operated pair-fed rats (PF, hatched bars), portacaval shunted rats (PCS-PF, cross hatched bars) and in rats with portacaval shunts and bile duct ligation (PCSBDL, filled bars). Data are means $\pm S E M$; $N=6$ to 10 per group. 
observed intestinal ammonia release in normal unoperated control rats was in close agreement with data previously reported by our group (19). From these data it seems that the (level of) hyperammonemia in both chronic liver insufficiency groups is mainly caused by the existence of a portasystemic shunt and not by enhanced intestinal ammonia production. Although the PCSBDL group undoubtedly has more severe parenchymal damage than the PCS-PF group, arterial ammonia levels were similar. This could easily be interpreted as evidence that the residual liver function is still capable in this PCSBDL model to detoxify total body ammoniagenesis. However, in a steady state situation as in our experiments, arterial ammonia levels do not give information about the rate of whole body ammoniagenesis and detoxification, except that these antagonistic processes are of equal magnitude. Thus, ammoniagenesis in other organs (e.g. the kidney) may be reduced or alternatively, as we have observed previously (unpublished observation, 1992), urinary ammonia excretion may be enhanced in the PCSBDL group.

At day 7 portal drained viscera ammonia production was less in PF than in PCS-PF rats. This deserves some specific comment. The decrease in portal drained viscera ammonia production in the PF group was mainly caused by a decreased portal vein plasma flow, since portal vein-arterial ammonia concentration differences (not shown) were similar in all operated groups. To exclude the possibility of an artificially decreased flow, by coincidence or technical failure, we repeated portal plasma flow measurements in a similar series of $5 \mathrm{PF}$ rats, treated and fed identically, and found the same value. Thus, we must assume that the decreased plasma flow in the PF group at day 7 is a true observation, for which we do not have an adequate explanation.

In PF as well as PCS-PF and PCSBDL rats, surgery and 7 days of semi-starvation resulted in an increase in arterial glutamine concentrations. However, no differences between the groups were observed on day 14 , despite substantial differences in arterial ammonia concentrations. These findings suggest that the regulation of arterial glutamine levels is more related to nutritional factors and/or surgical trauma than to hyperammonemia or liver insufficiency per se.

Although arterial glutamine levels were always higher in the PF, PCS-PF and PCSBDL groups than in normal control rats, portal drained viscera glutamine uptake in the PF, PCS-

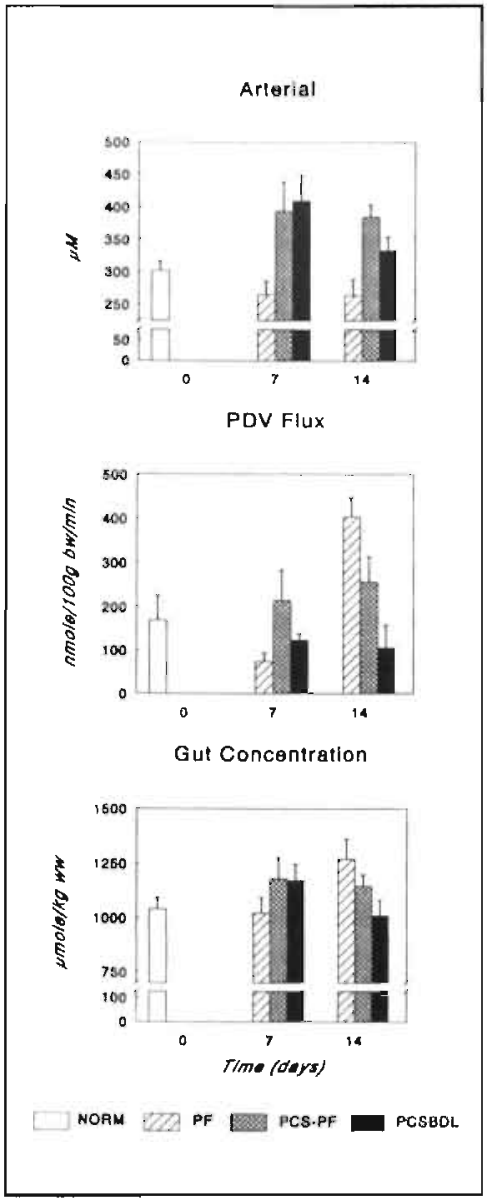

Figure 4. Alanine: arterial concentrations (upper panel; $\mu \mathrm{M}$ ), portal drained viscera fluxes (middle panel; nmole.100 g body $w^{-1} \mathrm{~min}^{-1}$ ) and jejunal tissue concentrations (lower panel; $\mu$ mole $\mathrm{kg}$ wet $\mathrm{wt}^{-1}$ ) in normal control rats (NORM, open bars), sham operated pair-fed rats (PF, hatched bars), portacaval shunted rats (PCS.PF, cross hatched bars) and in rats with portacaval shunts and bile duct ligation (PCSBDL, filled bars). Data are means $\pm S E M ; N=6$ to 10 per group. 
PF and PCSBDL groups never exceeded that in normal control rats. The observed portal drained viscera glutamine uptake in normal controls was similar to data recently reported by our group (19). Also, although the elevated arterial glutamine levels at day 7 in PCSBDL compared with PF rats resulted in increased portal drained viscera glutamine uptake, this was not accompanied by a significantly increased intestinal ammonia release. Finally, the fractional glutamine extraction of approximately $30 \%$ in normal controls (Table 3 ), closely resembling that reported by Windmueller $(7,8)$, decreased in the hyperammonemic groups. The hypothesis underlying this study was that hyperglutaminemia during chronic liver insufficiency would enhance intestinal glutamine consumption and subsequent ammonia release, thus aggravating the already existing portasystemic shunting-induced hyperammonemia. From the present data we conclude that this hypothesis does not apply to these chronic liver insufficiency-induced hyperammonemia models in the rat.

Several other interesting observations were made. Intestinal release of taurine in all other groups reversed to uptake in the PCSBDL group. This could indicate that in bile duct ligated rats, taurine is not excreted in the bile and therefore cannot be taken up from the luminal side, but instead is taken up from the blood. No data are available concerning the free taurine concentration of bile (33), but taurine can be assumed to be present in considerable amounts in bile as bile salt conjugates (34). It is likely that these bile salts can function as a source for intestinal taurine uptake. The fact that intestinal glycine release in PCSBDL rats was significantly decreased provides additional evidence that such a mechanism may be operative, because the part of bile salts not conjugated with taurine consists of glycine conjugates (34). The final metabolic explanation for the taurine taken up remains to be clarified.

The observation that intestinal glutamine uptake was less in the hyperammonemic and sham operated groups than in normal control rats, makes it of special interest to consider the fluxes of the most important nitrogenous breakdown products (other than ammonia) of intestinal glutamine breakdown: glutamate, alanine and citrul line $(7,28,35,36)$. Alanine release from the intestines decreased at day 14 in both hyperammonemic groups compared to sham operated rats, which seems to be compatible with the observed reduced intestinal glutamine uptake. Also, it was found that intestinal release of citrulline did not change importantly in hyperammonemic rats. This could 
implicate that other sources are also important precursors for citrulline release. Also, it seems that citrulline release is kept constant to provide precursors for renal arginine biosynthesis (28). Portal drained viscera glutamate fluxes did not significantly differ from zero in several groups and could only account for minimal amounts of glutamine uptake. This is compatible with literature data suggesting that glutamate, whether taken up from the luminal side or derived from mucosal glutamine breakdown, is completely utilized by the intestines (8).

The changes in jejunal tissue concentrations are difficult to interpret. The elevated tissue glutamine levels in both hyperammonemic groups suggest that glutamine breakdown in the glutaminase reaction is diminished, which seems supported by the decreased glutamine uptake and fractional glutamine extraction. However, this does not seem compatible with the simultaneously elevated levels of glutamate and ammonia, the products of the glutaminase reaction. Because intestinal glutamine synthetase activity is very low $(14,28)$, probably the only plausible alternative explanation for increased jejunal glutamine levels is enhanced intestinal protein breakdown. However, this should lead to enhanced intestinal release of essential amino acids, which was not observed either. Thus, presumably, the observed changes reflect the end result of a new equilibrium, in which elevated intestinal ammonia levels (13) partially inhibit mucosal glutaminase activity. The glutaminase reaction, yielding energy for the gut (28), can then only be driven at the expense of elevated tissue glutamine levels, provided by uptake of blood-derived glutamine. Also, in light of this, the decreased tissue BCAA levels in the PCSBDL group could be due to enhanced BCAA transamination, providing glutamate and $\alpha$-ketoglutarate for energy. Firm conclusions are, however, not justified based on the present data.

In the present experiments, portacaval anastomosis combined with common bile duct ligation was introduced as a relatively new model to study nitrogen metabolism during chronic liver insufficiency. Construction of a portacaval anastomosis alone induces, besides portasystemic shunting of gut-derived blood, a relative and absolute reduction of liver mass $(15-17,37)$. Urea synthesis capacity has been shown to be limited in portacaval shunted rats, probably related to the reduced liver mass $(15,16)$. To these effects of portacaval anastomosis alone, the effects of chronic bile duct ligation were added. Prolonged common bile duct ligation induces periportal fibrosis, portal hypertension and portasystemic shunting $(29,31)$ as well as metabolic dysfunction of the liver $(30,31)$. Although the resulting jaundice is of a different etiology than that commonly encountered in cirrhotic patients, it is also cholestatic and therefore introduces the impaired cell-mediated immunity and Kupffer cell dysfunction (30-32) in the model of portacaval shunting/biliary obstruction. Because portacaval shunted/biliary obstructed rats showed subtle signs pointing to more pronounced hepatic encephalopathy, we think that the portacaval shunt/biliary obstruction model could be useful in future studies on the pathophysiology of chronic liver insufficiency and the related hepatic encephalopathy.

In conclusion, these experiments show that under conditions of standardized food intake, portal drained viscera ammonia generation remained unchanged in rats with chronic liver insufficiency despite alterations in arterial glutamine and intestinal glutamine uptake. This suggests that arterial ammonia levels in these rats are mainly determined by the existence of portasystemic shunting, and not by arterial glutamine levels, intestinal glutamine uptake or ammonia liberation. Finally, portacaval shunting combined with common bile duct ligation 
appears to be a useful model to study nitrogen metabolism during chronic liver insufficiency-induced mild hyperammonemia in the rat.

\section{REFERENCES}

1. Record CO. Neurochemistry of hepatic encephalopathy, Gut 1991;32:1261-1263.

2. Weber FL, Veach GL. The importance of the small intestine in gut ammonium production in the fasting dog. Gastroenterology 1979;77:235-240.

3. Weber FL, Friedman DW, Fresard KM. Ammonia production from intraluminal amino acids in canine jejunum. Am J Physiol 1988;254:G264-G268.

4. Imler M, Schlienger J-L, Chabrier G, Comte F. Origine rénale de l'hyperammoniémie provoquee par un régime hyperprotidique chez le rat normal ou porteur d'une stricture portale. Gastroenterol Clin Biol 1983;7:740-745.

5. Imler M, Schlienger J-L, Chabrier G, Simon C. Arterial ammonia changes of renal origin induced in the rat by acid and alkaline diets. Res Exp Med 1986;186:353-363.

6. Imler M, Schlienger J-L. The effect of chronic uremia on portal and systemic ammonemia in normal and poral-strictured rats. J Lab Clin Med 1979;94:872-878.

7. Windmueller HG, Spaeth AE. Uptake and metabolism of plasma glutamine by the small intestine. $\mathrm{J}$ Biol Chem 1970;249:5070-5079.

8. Windmueller HG, Spaeth AE. Intestinal metabolism of glutamine and glutamate from the lumen as compared to glutamine from blood. Arch Bioch Bioph 1975;171:662-672.

9. Zieve L. Pathogenesis of hepatic encephalopathy. Metabolic Brain Dis 1987;2:147-165.

10. Meijer AJ, Lamers WH, Chamuleau RAFM. Nitrogen metabolism and omithine cycle function. Physiol Rev 1990;70:701-748.

11. Kaiser S, Gerok W, Haüssinger D. Ammonia and glutamine metabolism in human liver slices:new aspects on the pathogenesis of hyperammonaemia in chronic liver disease. Eur $\mathrm{J}$ Clin Invest 1988; 18:535-542.

12. Gjedde A, Lockwood AH, Duffy TE, Plum F. Cerebral blood flow and metabolism in chronically hyperammonemic rats:Effect of an acute ammonia challenge. Ann Neurol 1978;3:325-330.

13. Newsholme EA, Leech AR. Biochemistry for the Medical sciences. John Wiley \& Sons, New York, 1983.

14. Lund P. A radiochemical assay for glutamine synthetase, and activity of the enzyme in rat tissues. Bioch J 1970;118:35-39.

15. Brewer TG, Berry WR, Harmon JW, Walker SH, Dunn MA. Urea synthesis after protein feeding reflects hepatic mass in rats. Hepatology 1984:4:905-911.

16. Steele RD. Hyperammonemia and orotic aciduria in portacaval shunted rats. J Nutr 1984; 114:210-216.

17. Benjamin IS, Engelbrecht GHC, Saunders SJ, van Hoom Hickman R. Amino acid imbalance following portal diversion in the rat. The relevance of nutrition and of hepatic function. J Hepatology 1988;7:208-214.

18. Lockwood AH, McDonald JM, Reiman RE, Gelbard AS, Laughlin JS, Duffy TE, Plum F. The dynamics of ammonia metabolism in man. Effects of liver disease and hyperammonemia. J Clin Invest 1979;63:449-460.

19. Dejong CHC, Kampman MT, Deutz NEP, Soeters PB. Altered glutamine metabolism in rat portal drained viscera and hindquarter during hyperammonemia. Gastroenterology 1992;102:936-948.

20. Dejong CHC, Kampman MT, Deutz NEP, Soeters PB. Cerebral cortex ammonia and glutamine metabolism during liver insufficiency-induced hyperammonemia in the rat. J Neurochem 1992;59:1071-1079.

21. Dejong CHC, Deutz NEP, Soeters PB. Inter-organ nitrogen exchange during prolonged starvation in the rat. J Clin Nutr Gastroenterol 1991;6: 176-183.

22. Maddison JE, Dodd PR, Morrison M, Johnston GAR, Farrell GC. Plasma GABA, GABA-like activity and the brain GABA-benzodiazepine receptor complex in rats with chronic hepatic encephalopathy. Hepatology 1987:7:621-628.

23. de Boer JEG, Oostenbroek RJ, van Dongen JJ, Janssen MA, Soeters PB. Sequential metabolic characteristics following portacaval shunts in rats. Eur Surg Res 1986; 18:96-106. 
24. Deutz NEP, Dejong CHC, Athanasas G, Soeters PB. Partial enterectomy in the rat does not diminish muscle glutamine production. Metabolism 1992; in press.

25. Dejong CHC, Deutz NEP, Soeters PB. A simple new method for repeated in vivo cerebral cortex flux measurement in rats. Lab Anim Sci 1992;42:280-285.

26. van Eijk HMH, van der Heijden MAH, van Berlo CLH, Soeters PB. Fully automated liquid-chromatographic determination of amino acids. Clin Chem 1988;34:2510-2513.

27. Ribeiro J, Nordlinger B, Ballet F, Cynober L, Coudray-Lucas C, Baudrimont M, Legendre C. Delelo R, Panis Y. Intrasplenic hepatocellular transplantation corrects hepatic encephalopathy in portacaval shunted rats. Hepatology 1992;15:12-18.

28. Windmueller HG. Glutamine utilization by the small intestine. Adv Enzyme 1982;53:210-237.

29. Kountouras J, Billing BH, Scheuer PJ. Prolonged bile duct obstruction:a new experimental model for cirrhosis in the rat. Br J Exp Path 1984;65:305-311.

30. Greve JW, Gouma DJ, Soeters PB, Buurman WA. Suppression of cellular immunity in obstructive jaundice is caused by endotoxins. A study in germ-free rats. Gastroenterology 1990;98:478-485.

31. Dunn CW. Horton JW, Megison SM, Vuitch MF. Contribution of portal systemic shunt to Kupffer cell dysfunction in obstructive jaundice. J Surg Res 1991;50:234-239.

32. Roughneen PT, Gouma DJ, Kulkarni AD, Fanslow WF, Rowlands BJ. Impaired specific cellmediated immunity in experimental biliary obstruction and its reversibility by intemal biliary drainage. J Surg Res 1986;41:113-125.

33. Fölsch UR, Wormsley KG. The amino acid composition of rat bile. Experientia 1977;33:1055-1056.

34. Huxtable RJ. Physiological actions of taurine. Physiol Rev 1992;72:101-163.

35. Windmueller HG, Spaeth AE. Respiratory fuels and nitrogen metabolism in vivo in small intestine of fed rats. J Biol Chem 1980;255:107-112.

36. Windmueller HG, Spaeth AE. Identification of ketone bodies and glutamine as the major fuels in vivo for postabsorptive rats small intestine. J Biol Chem 1978;253:69-76.

37. Dunlop DS, Kaufman H, Zanchin G, Lajtha A. Protein synthesis rates in rats with portacaval shunts. J Neurochem 1984;43:1487-1489. 



\title{
Cerebral cortex during chronic liver failure ${ }^{1}$
}

To be published as: C.H.C. Dejong. N.E.P. Deutz, P.B. Soeters. Cerebral conex ammonia and glutamine metabolism in two rat models of chronic liver insufficiency-induced hyperammonemia: influence of pair-feeding. J Neurochem 1993;60:in press.

\begin{abstract}
Enhanced cerebral cortex ammonia uptake, subsequent glutamine synthesis and glutamine release into the blood stream has been hypothesized to deplete cerebral cortex glutamate pools. We investigated this hypothesis in rats with chronic liver insufficiency-induced hyperammonemia and in pair-fed controls to rule out effects of differences in food intake. Cerebral cortex plasma flow and venous-arterial concentration differences of ammonia and amino acids, as well as cerebral cortex tissue concentrations, were studied 7 and 14 days after surgery in portacaval shunted/bile duct ligated, portacaval shunted, and in sham operated rats, while the latter two were pair-fed to the first group, and in normal unoperated ad libitum fed control rats. At both time points, arterial ammonia was elevated in the chronic liver insufficiency groups and arterial glutamine was elevated in portacaval shunt/biliary obstruction rats compared to the other groups. In the chronic liver insufficiency groups net cerebral cortex ammonia uptake was observed at both time points, and was accompanied by net glutamine release. Also in these groups, cerebral cortex tissue glutamine, many other amino acids and ammonia levels were elevated. Tissue glutamate levels were decreased to a similar level in all operated groups compared with normal unoperated rats, irrespective of plasma and tissue ammonia and glutamine levels. These results demonstrate that during chronic liver insufficiency-induced hyperammonemia the rat cerebral cortex enhances net ammonia uptake and glutamine release. However, the decrease in tissue glutamate concentrations in these chronic liver insufficiency models seems primarily related to nutritional status and/or surgical trauma.
\end{abstract}

\footnotetext{
'This chapter is an exact copy of the original publication and consequently does not present all data of the experiments. Additional data are available on request.
} 


\section{INTRODUCTION}

Ammonia is still considered to be an important factor in the pathogenesis of hepatic encephalopathy (1-4). The etiological role of ammonia in this neuropsychiatric syndrome has been explained in the concept of 'portasystemic encephalopathy' (for recent review, see (4)). According to this concept, ammonia arising in the intestines escapes hepatic clearance during liver insufficiency as a consequence of intra- or extrahepatic portasystemic shunting and probably also reduced hepatic capacity of urea synthesis and glutamine synthetase (GS'; EC 6.3.1.2) (2,5-7), thereby leading to hyperammonemia. Because the blood-brain barrier is freely permeable to ammonia, elevated arterial ammonia levels would then, according to this hypothesis, lead to enhanced cerebral ammonia uptake, subsequent incorporation of ammonia into glutamine in the GS reaction, and glutamine release into the blood $(5,8,9)$. Because ammonia at elevated levels is toxic to the central nervous system (10-12); for reviews, see: $(3,5,9))$, whereas glutamine is a non-toxic compound, the GS reaction has the beneficial effect of transforming a toxic compound into a non-toxic one. On the other hand, because this reaction consumes glutamate, the principal excitatory neurotransmitter in the brain, it has been hypothesized to result in disturbed glutamatergic neurotransmission, contributing to the neuro-inhibition of hepatic encephalopathy $(4,9,13)$.

The general acceptance of this concept has been hampered by the difficulty to demonstrate net ammonia uptake by the brain (1). Although unidirectional brain ammonia uptake has been repeatedly observed in various hyperammonemic states by using tracers $(5,9,13,14)$, net ammonia uptake by the brain has been found much more difficult to prove. This discrepancy between the unidirectional forward flux data and the net arteriovenous concentration difference measurements has been explained by assuming that the brain continuously uses and produces ammonia (1).

Another prerequisite for the validity of the glutamate depletion theory is the net loss of glutamate from the brain, by net glutamine release into the blood stream. Although there is no doubt that cerebral tissue glutamine levels are elevated during hyperammonemia $(3-5,9)$, it has been subject to debate whether this will always lead to net glutamine release from the brain (reviewed in: $(9,15)$ ). Reports on this subject are scarce and limited to chronic portacaval shunting-induced hyperammonemia (8). In this context, however, little attention has been paid to the fact that malnutrition and starvation per se already lead to glutamine release from several organs (16). Yet, it seems likely that the brain also removes excess nitrogen as glutamine $(9,15)$.

Recently, we observed net ammonia uptake by the cerebral cortex in $48 \mathrm{~h}$ portacaval shunted rats and in rats with acute liver ischemia, providing new evidence for a role of the brain (or brain regions) in net ammonia removal from the circulation during liver disease (17). Also, this was accompanied by net glutamine release during acute liver ischemia, and by decreased cerebral cortex tissue glutamate concentrations in portacaval shunted rats and rats with acute liver ischemia. Although this seemingly supported the 'glutamate depletion theory', there were no differences in tissue glutamate levels between portacaval shunted and acute liver ischemia rats, despite tremendous differences in arterial and tissue ammonia and glutamine levels as well as hepatic encephalopathy stage (17). This suggested that the 'glutamate depletion theory' in its most primitive form (ammonia in - glutamine out - tissue glutamate decrease) is not valid, and prompted us to further explore cerebral ammonia and 
glutamine metabolism during liver failure. Because the experiments during acute hyperammonemia bear little resemblance with the clinical situation, we undertook to investigate cerebral cortex ammonia and glutamine metabolism during chronic liver insufficiency.

Although portacaval shunting (PCS) is a well-known, reproducible model for chronic liver insufficiency-induced hyperammonemia and hepatic encephalopathy (18-20), portacaval shunting combined with common bile duct ligation was recently suggested to be a more reliable, new model for chronic hyperammonemia, inducing a more pronounced clinical degree of hepatic encephalopathy than PCS alone (21). Therefore, the present studies were performed in these two models of chronic liver insufficiency: portacaval shunting, and portacaval shunting plus bile duct ligation, as well as in sham operated and normal unoperated control rats. Ammonia and amino acid exchange across the cerebral cortex were assessed by measuring plasma flows and venous-arterial concentration differences (17). In addition, cerebral cortex tissue ammonia and amino acid concentrations were measured. To reduce the effects of differences in food intake, sham operated and portacaval shunted rats were pair-fed with the anorectic portacaval shunt/biliary obstruction group.

The present study demonstrates that moderate hyperammonemia leads to net cerebral cortex ammonia uptake, to ammonia and glutamine accumulation within the cerebral cortex, and finally to glutamine release into the blood. However, the decreased total cerebral cortex tissue glutamate levels in the present experiments appear to be primarily related to diminished food intake and/or surgical trauma.

\section{MATERIALS AND METHODS}

Materials Intramedic ${ }^{R}$ Polyethylene (PE) Tubing was obtained from Clay Adams (Parsippany, NJ, U.S.A.). Para-aminohippuric acid (PAH) was obtained from Sigma Chemicals (St. Louis, MO, U.S.A.).

Animals Male specified pathogen free Wistar rats ( $n=53,300 \pm 25 \mathrm{~g}$ ) were used throughout and were housed under standard conditions (12/12 h light/dark cycle) and received standard lab chow and water ad libitum until entering the experiments i.e. until surgery. Rats were maintained in accordance with the recommendations of the Guide for the Care and Use of Laboratory Animals, as applied in our institute. All surgical procedures were performed by licensed personnel under the auspices of the licensing authority of the Centralized Animal Facilities of our institute.

Groups Four groups were studied: 1 PCSBDL group. Portacaval shunting (PCS) plus bile duct ligation (BDL) in one surgical session. 2 PCS-PF group. Portacaval shunting. $\underline{3} P F$ group. Laparotomy and manipulations as in PCS-PF rats, but without shunting (sham operation). 4 NORM group. Sampling without previous surgery; normal control rats, receiving food ad libitum.

Before surgery, rats were randomly assigned to one of the groups. Surgical procedures were of equal duration in PCS-PF and PF rats and their individual PCSBDL mate. After resuscitation from surgery, PCSBDL, PCS-PF and PF rats were placed in metabolic cages. PCSBDL rats had free access to standard pellet food. In these rats, daily food intake was recorded and this amount was administered to fixed PCS-PF and PF mates to eliminate 
effects of differences in food intake (pair-feeding: PF). In the PCSBDL, PCS-PF and PF groups blood was sampled 7 or 14 days after surgery (see below). Body weight was measured at the time intervals indicated in the figures. Water was administered ad libitum in all groups.

Behavior Before entering the experiments and before sampling, behavior of all rats was studied during a $5 \mathrm{~min}$ period. In this behavioral assessment, specific attention was directed towards appearance, presence of lethargy, spontaneous locomotor activity and exploratory behavior (standing upright etc.), presence of tiptoe gait, hindlimb toeing out and arched back, reaction to tail pinching and presence of ataxia. Since the PCSBDL rats were jaundiced, this staging procedure could not be performed in a blind fashion.

Surgery Operations and sampling procedures were carried out under ether anesthesia. Portacaval shunting was performed by the button technique, as described previously (22). Bile duct ligation was performed in the same session as portacaval shunting by double ligation near the liver and single ligation near the duodenum after which the piece of bile duct between the ligatures was resected. In the PCS-PF and PF group the bile duct was manipulated, but not dissected, to avoid stenosis.

Sampling Prior to sampling all rats were overnight fasted. Rectal temperature was maintained at $37 \pm 1^{\circ} \mathrm{C}$ during the sampling procedure. Venous-arterial concentration differences and plasma flows across the cerebral cortex were measured as described previously $(17,23)$. Briefly, a heparinized PE 10 catheter was inserted via the femoral artery into the abdominal aorta and two heparinized PE 10 catheters were inserted into the superior sagittal sinus. To accomplish this, the skull was exposed and the bone was milled away anterior to the bregma and posterior to the lambdoid suture, exposing $5 \mathrm{~mm}$ of the anterior and $5 \mathrm{~mm}$ of the posterior part of the superior sagittal sinus. Then, the anterior catheter was inserted, its tip being positioned at the bregma, and the posterior catheter was inserted in the confluence of sinuses. Both catheters were fixed with cyanoacrylate adhesive. The anterior catheter was used for PAH infusion for flow determinations, the posterior catheter for blood sampling.

An iso-osmolar, $\mathrm{pH}$ adjusted, $5 \mathrm{mM} \mathrm{PAH}$ solution was infused in the anterior superior sagittal sinus catheter at a rate of $30 \mu \mathrm{L} \mathrm{min}^{-1}$, after a $0.15 \mathrm{~mL} / 100 \mathrm{~g}$ body wt priming dose of a $50 \mathrm{mM}$ PAH solution via the arterial catheter, allowing for $20 \mathrm{~min}$ equilibration prior to blood sampling. At the end of the equilibration period, $1 \mathrm{~mL}$ of blood from the confluence of sinuses and the femoral artery were simultaneously collected on ice. Venous blood was sampled semi-automatically at rate of $50 \mu \mathrm{L} \mathrm{min}^{-1}$ in heparinized cups. Arterial samples were collected free flowing. Blood samples were kept on ice during further processing. After blood sampling the cortex of one hemisphere was immediately freeze-clamped in liquid nitrogen and stored at $-70^{\circ} \mathrm{C}(17)$. This procedure was accomplished within $1 \mathrm{~min}$, warranting reliable tissue ammonia and amino acid determinations, especially for $\gamma$ aminobutyric acid (GABA) $(17,24)$.

Biochemical analysis Plasma for ammonia, urea and amino acids was obtained by whole blood centrifugation. Centrifugations were performed at $4^{\circ} \mathrm{C}$ during $5 \mathrm{~min}$ at $8,800 \mathrm{~g}$. Plasma was put into liquid nitrogen within $20 \mathrm{~min}$. All samples were stored at $-70^{\circ} \mathrm{C}$. Hematocrit was obtained with a microfuge. For tissue ammonia and amino acid determinations, 5\% $(\mathrm{w} / \mathrm{v})$ sulfosalicylic acid extracts were prepared (22). Ammonia, urea, lactate and glucose were determined by standard enzymatic methods (16), amino acids were determined by HPLC technique (25). PAH was determined spectrophotometrically as described previously 
(23). Total bilirubin was determined in plasma with a commercially available test kit (Hoffmann-La Roche, Basel, Switzerland). Water content was determined by freeze-drying (17). The coefficient of variance for all determinations was below $4 \%$ (e.g. $2 \%$ for glutamine, $4 \%$ for ammonia and $2 \%$ for urea and bilirubin) $(22,25)$.

Calculations Venous (confluence) concentrations were corrected for the dilution by the PAH infusion. $\alpha$ Amino nitrogen was calculated as the sum of amino acids measured (25). Urea values were corrected for ammonia. Plasma PAH concentration was calculated as $[\mathrm{PAH}]_{\text {plasma }}=[\mathrm{PAH}]_{\text {blood }} \cdot 100 /(100-\mathrm{Ht})$. Fluxes $\left(\mathrm{nmole} \mathrm{min}^{-1}\right)$ were calculated as flow times V-A difference. A positive figure means net efflux, negative values reflect net uptake. Plasma flows were calculated as $F_{s s s}=I /\left([P A H]_{C}-[P A H]_{A}\right)(23)$. In this formula, $F_{S s s}$ is superior sagittal sinus plasma flow $\left(\mathrm{mL} \mathrm{min}^{-1}\right)$, $\mathrm{I}$ is the infused PAH $\left(\mu\right.$ mole $\left.\mathrm{min}^{-1}\right)$, and $[\mathrm{PAH}]_{\mathrm{CA})}$ is the confluence (C) and arterial (A) plasma PAH concentration $(\mu \mathrm{M})$, respectively.

Statistical analysis Statistics were performed using the SPSS/PC+ Statistical Software Package, version 3.1 (SPSS Inc., Chicago, IL., USA). Data presented are means $\pm \mathrm{SEM}, \mathrm{P}<0.05$ was considered significant. Analysis of variance was used to test for group effects (ANOVA) and time effects within groups (ONEWAY). The

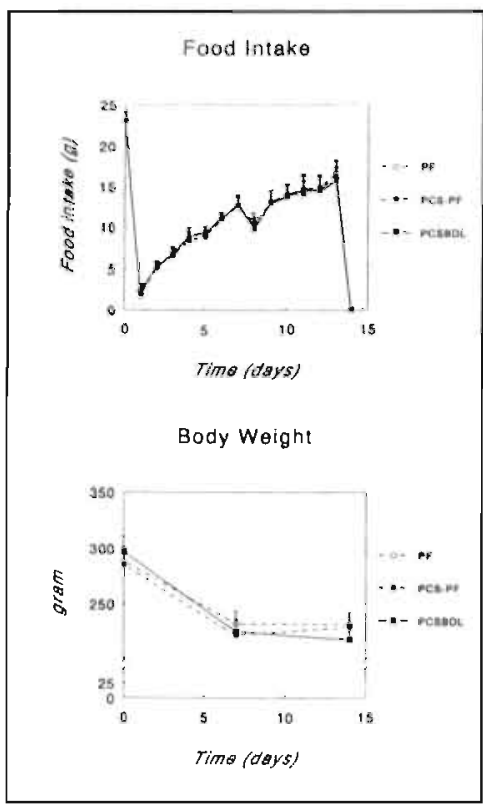

Figure 1. Food intake (upper panel) and body weight (lower panel) in PCSBDL (filled squares), PCS-PF (filled circles) and PF (open circles) rats during 14 days of pairfeeding. The plotted individual curves are means \pm SEM of 14 to 16 rats in the first 7 days and 7 or 8 rats between day 7 and 14 . Mann-Whitney $U$ non-parametric test was used for differences between groups at specific time points. Wilcoxon's nonparametric test was used for differences from zero. Fischer's exact test was used to test for differences in encephalopathy stages (one-tailed, binomial testing). Significances are indicated in Tables 1-4.

\section{RESULTS}

General Food intake and body weight (Figure 1) were similar in PCSBDL, PCS-PF and PF rats during the whole experiment. No signs of systemic or local inflammation or impaired wound healing were observed in PCSBDL rats. Arterial total bilirubin (Table 1) was slightly increased in PCS-PF compared with PF rats, as previously reported (20), and strongly elevated in PCSBDL rats compared with all other groups.

Behavior No obvious signs of encephalopathy were observed in PCS-PF compared with PF rats. All PCSBDL rats had a characteristic ill-groomed 'gerbil' appearance with bristly hair. Also, in these rats subtle signs were observed pointing to more pronounced encephalopathy, such as decreased spontaneous locomotor activity and exploratory behavior, more abnormalities in gait and hindlimb ataxia. 
Table 3. Cerebral cortex fluxes and plasma flow.

\begin{tabular}{|c|c|c|c|c|c|c|c|c|c|}
\hline & 0 & & 7 & & & & 14 & & \\
\hline & NORM & PF & PCS.PF & PCSBDL & & $P F$ & PCS-PF & PCSBDL & \\
\hline ow & $209 \pm 47$ & $143 \pm 24$ & $174 \pm 45$ & $171 \pm 51$ & & $184 \pm 52$ & $175 \pm 70$ & $167 \pm 23$ & \\
\hline LC & $-112 \pm 69$ & $-135 \pm 44$ & $-158 \pm 38$ & $-146 \pm 34$ & & $-89 \pm 62$ & $-112 \pm 53$ & 34 & \\
\hline$A C$ & $71 \pm 48$ & $-21 \pm 27$ & $9 \pm 16$ & $35 \pm 30$ & & $15 \pm 15$ & $5 \pm 15$ & 101 & \\
\hline MM & $0.6 \pm 0.8$ & $-0.6 \pm 0.7$ & $-4.1 \pm 1.4$ & $-4.0 \pm 1.2$ & 0 & $-0.2 \pm 1.0$ & $-1.0 \pm 0.6$ & 1.2 & $\infty$ \\
\hline U & $0.1 \pm 0.3$ & $-0.6 \pm 0.9$ & $2.0 \pm 0.8$ & $1.8 \pm 1.1$ & . & $-0.1 \pm 0.3$ & $-0.3 \pm 0.2 \approx$ & 0.2 & \\
\hline LN & $0.1 \pm 1.6$ & $0.5 \pm 1.0^{\circ}$ & $5.8 \pm 1.3^{n}$ & $6.4 \pm 2.6$ & & $-1.9 \pm 2.1$ & $2.8 \pm 0.8 \infty$ & 1.3 & $\infty$ \\
\hline$R$ & $0.2 \pm 0.2$ & $0.2 \pm 0.2$ & $0.7 \pm 0.1$ & 0.3 & . & $0.2 \pm 0.2$ & $0.4 \pm 0.1$ & 0.2 & \\
\hline HE & $0.0 \pm 0.2$ & $0.4 \pm 0.4$ & $0.6 \pm 0.3$ & 0.6 & - & $0.1 \pm 0.1$ & $0.3 \pm 0.1$ & 0.2 & h \\
\hline AA & $-0.9 \pm 1.9$ & $1.6 \pm 0.9$ & $1.4 \pm 0.8$ & $-1.0 \pm 0.9$ & $=$ & $.0 .4 \pm 1.0^{-}$ & $0.7 \pm 0.5$ & 0.8 & s \\
\hline LA & $0.3 \pm 0.4$ & $0.6 \pm 0.5$ & $1.2 \pm 0.3$ & $0.8 \pm 0.8$ & - & $0.3 \pm 0.3$ & $0.7 \pm 0.3$ & $2.0 \pm 0.4$ & \\
\hline$\alpha$ AN & $-8.9 \pm 10.3$ & $-5.4 \pm 4.8$ & $11.6 \pm 10.6$ & $7.1 \pm 5.9$ & 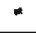 & $-14.4 \pm 8.1$. & $9.1 \pm 3.8^{\mathrm{bo}}$ & $6.8 \pm 9.2$ & 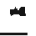 \\
\hline
\end{tabular}

Data are means \pm SEM in nmole $\min ^{-1}$, except plasma flows in $\mu \mathrm{Lmin}{ }^{-1}$. For significance symbols, see Table 1 . The remaining amino acid fluxes were not significantly different from zero in either all or most groups.

Table 4. Cerebral cortex tissue concentrations and water content.

\begin{tabular}{|c|c|c|c|c|c|c|c|c|}
\hline & DAY 0 & & DAY 7 & & & DAY 14 & & \\
\hline & NORM & $\mathrm{PF}$ & PCS-PF & PCSBDL & PF & PCS-PF & PCSBDL & \\
\hline AMM & $794 \pm 34$ & $776 \pm 39$ & $1095 \pm 63$ & $n \quad 1141 \pm 55^{n}$ & $757 \pm 42$ & $968 \pm 38$ ch & $1059 \pm 67$ & at \\
\hline $\mathrm{H}_{2} \mathrm{O}$ & $78.3 \pm 0.2$ & $78.7 \pm 0.3$ & $78.8 \pm 0.1$ & $78.7 \pm 0.4$ & $78.2 \pm 0.6$ & $78.5 \pm 0.4$ & $78.4 \pm 0.3$ & \\
\hline SPP & $2300 \pm 94$ & $2180 \pm 80$ & $1947 \pm 120$ & $2054 \pm 91$ & $2387 \pm 119$ & $2042 \pm 72$ & $2109 \pm 106$ & 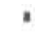 \\
\hline GLU & $11828 \pm 197$ & $11204 \pm 130 m$ & $11158 \pm 296$ & $11039 \pm 320$ & $10895 \pm 329$ & $10814 \pm 286$ & $10877 \pm 339$ & \\
\hline LN & $5501 \pm 110$ & $5554 \pm 216$ & $8352 \pm 499$ & $8899 \pm 591^{n}$ & $5248 \pm 169$ & $6809 \pm 403^{\text {cho }}$ & $8428 \pm 444$ & \\
\hline GLY & $682 \pm 13$ & $796 \pm 45$ & $840 \pm 49$ & $892 \pm 34^{\circ}$ & $750 \pm 17$ & $780 \pm 9$ & $879 \pm 32$ & - \\
\hline$A B A$ & $1527 \pm 54$ & $1599 \pm 126$ & $1496 \pm 96$ & $1786 \pm 133$ & $1607 \pm 105$ & $1434 \pm 97^{\circ}$ & $1533 \pm 79$ & \\
\hline YR & $87 \pm 6$ & $80 \pm 3$ & $141 \pm 9$ & $203 \pm 13^{k x}$ & $83 \pm 4$ & $141 \pm 11^{\text {ch }}$ & $203 \pm 9$ & anis \\
\hline PHE & $74 \pm 4$ & $95 \pm 10$ & $123 \pm 7$ & $189 \pm 16^{*}$ & $84 \pm 6$ & $126 \pm 14^{\text {th }}$ & $159 \pm 10$ & $m$ \\
\hline RP & $23 \pm 2$ & $22 \pm 3$ & $33 \pm 2$ & $53 \pm \quad 6$ & $24 \pm 1$ & $41 \pm 4^{a}$ & $52 \pm 2$ & mis \\
\hline BCAA & $329 \pm 7$ & $343 \pm 8$ & $332 \pm 11$ & $338 \pm 29$ & $317 \pm 15$ & $312 \pm 16$ & $319 \pm 11$ & \\
\hline$A A$ & $162 \pm 9$ & $175 \pm 11$ & $264 \pm 12$ & $392 \pm 24$ & $168 \pm 8$ & $267 \pm 23^{\text {का }}$ & $362 \pm 16$ & chis \\
\hline AN & $2270 \pm 250$ & $32106 \pm 664$ & $34427 \pm 576$ & - $35986+1023^{\circ}$ & $31352 \pm 440$ & $31961 \pm 532$ & $33869 \pm 828$ & \\
\hline
\end{tabular}

Data are expressed as mean $\pm S E M$ in $\mu$ mole kg wet weight ${ }^{1}(w w)$. For significance symbols, see Table 1. GABA: $\gamma$ aminobutyric acid.

Glutamine (Figure 3; Table 1, 3\&4) Arterial glutamine concentrations (Figure 3, upper panel) were significantly higher in PCSBDL compared with all other groups, but no major differences were observed between the PCS-PF, PF and NORM group. Cerebral cortex glutamine flux (Figure 3, middle panel) did not significantly differ from zero in the NORM and $\mathrm{PF}$ group. However, in both hyperammonemic groups glutamine was released in 
Table 3. Cerebral cortex fluxes and plasma flow.

\begin{tabular}{|c|c|c|c|c|c|c|c|c|c|}
\hline & 0 & & 7 & & & & 14 & & \\
\hline & NORM & PF & PCS.PF & PCSBDL & & $P F$ & PCS-PF & PCSBDL & \\
\hline ow & $209 \pm 47$ & $143 \pm 24$ & $174 \pm 45$ & $171 \pm 51$ & & $184 \pm 52$ & $175 \pm 70$ & $167 \pm 23$ & \\
\hline LC & $-112 \pm 69$ & $-135 \pm 44$ & $-158 \pm 38$ & $-146 \pm 34$ & & $-89 \pm 62$ & $-112 \pm 53$ & 34 & \\
\hline$A C$ & $71 \pm 48$ & $-21 \pm 27$ & $9 \pm 16$ & $35 \pm 30$ & & $15 \pm 15$ & $5 \pm 15$ & 101 & \\
\hline MM & $0.6 \pm 0.8$ & $-0.6 \pm 0.7$ & $-4.1 \pm 1.4$ & $-4.0 \pm 1.2$ & 0 & $-0.2 \pm 1.0$ & $-1.0 \pm 0.6$ & 1.2 & $\infty$ \\
\hline U & $0.1 \pm 0.3$ & $-0.6 \pm 0.9$ & $2.0 \pm 0.8$ & $1.8 \pm 1.1$ & . & $-0.1 \pm 0.3$ & $-0.3 \pm 0.2 \approx$ & 0.2 & \\
\hline LN & $0.1 \pm 1.6$ & $0.5 \pm 1.0^{\circ}$ & $5.8 \pm 1.3^{n}$ & $6.4 \pm 2.6$ & & $-1.9 \pm 2.1$ & $2.8 \pm 0.8 \infty$ & 1.3 & $\infty$ \\
\hline$R$ & $0.2 \pm 0.2$ & $0.2 \pm 0.2$ & $0.7 \pm 0.1$ & 0.3 & . & $0.2 \pm 0.2$ & $0.4 \pm 0.1$ & 0.2 & \\
\hline HE & $0.0 \pm 0.2$ & $0.4 \pm 0.4$ & $0.6 \pm 0.3$ & 0.6 & - & $0.1 \pm 0.1$ & $0.3 \pm 0.1$ & 0.2 & h \\
\hline AA & $-0.9 \pm 1.9$ & $1.6 \pm 0.9$ & $1.4 \pm 0.8$ & $-1.0 \pm 0.9$ & $=$ & $.0 .4 \pm 1.0^{-}$ & $0.7 \pm 0.5$ & 0.8 & s \\
\hline LA & $0.3 \pm 0.4$ & $0.6 \pm 0.5$ & $1.2 \pm 0.3$ & $0.8 \pm 0.8$ & - & $0.3 \pm 0.3$ & $0.7 \pm 0.3$ & $2.0 \pm 0.4$ & \\
\hline$\alpha$ AN & $-8.9 \pm 10.3$ & $-5.4 \pm 4.8$ & $11.6 \pm 10.6$ & $7.1 \pm 5.9$ & 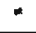 & $-14.4 \pm 8.1$. & $9.1 \pm 3.8^{\mathrm{bo}}$ & $6.8 \pm 9.2$ & 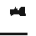 \\
\hline
\end{tabular}

Data are means \pm SEM in nmole $\min ^{-1}$, except plasma flows in $\mu \mathrm{Lmin}{ }^{-1}$. For significance symbols, see Table 1 . The remaining amino acid fluxes were not significantly different from zero in either all or most groups.

Table 4. Cerebral cortex tissue concentrations and water content.

\begin{tabular}{|c|c|c|c|c|c|c|c|c|}
\hline & DAY 0 & & DAY 7 & & & DAY 14 & & \\
\hline & NORM & $\mathrm{PF}$ & PCS-PF & PCSBDL & PF & PCS-PF & PCSBDL & \\
\hline AMM & $794 \pm 34$ & $776 \pm 39$ & $1095 \pm 63$ & $n \quad 1141 \pm 55^{n}$ & $757 \pm 42$ & $968 \pm 38$ ch & $1059 \pm 67$ & at \\
\hline $\mathrm{H}_{2} \mathrm{O}$ & $78.3 \pm 0.2$ & $78.7 \pm 0.3$ & $78.8 \pm 0.1$ & $78.7 \pm 0.4$ & $78.2 \pm 0.6$ & $78.5 \pm 0.4$ & $78.4 \pm 0.3$ & \\
\hline SPP & $2300 \pm 94$ & $2180 \pm 80$ & $1947 \pm 120$ & $2054 \pm 91$ & $2387 \pm 119$ & $2042 \pm 72$ & $2109 \pm 106$ & 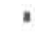 \\
\hline GLU & $11828 \pm 197$ & $11204 \pm 130 m$ & $11158 \pm 296$ & $11039 \pm 320$ & $10895 \pm 329$ & $10814 \pm 286$ & $10877 \pm 339$ & \\
\hline LN & $5501 \pm 110$ & $5554 \pm 216$ & $8352 \pm 499$ & $8899 \pm 591^{n}$ & $5248 \pm 169$ & $6809 \pm 403^{\text {cho }}$ & $8428 \pm 444$ & \\
\hline GLY & $682 \pm 13$ & $796 \pm 45$ & $840 \pm 49$ & $892 \pm 34^{\circ}$ & $750 \pm 17$ & $780 \pm 9$ & $879 \pm 32$ & - \\
\hline$A B A$ & $1527 \pm 54$ & $1599 \pm 126$ & $1496 \pm 96$ & $1786 \pm 133$ & $1607 \pm 105$ & $1434 \pm 97^{\circ}$ & $1533 \pm 79$ & \\
\hline YR & $87 \pm 6$ & $80 \pm 3$ & $141 \pm 9$ & $203 \pm 13^{k x}$ & $83 \pm 4$ & $141 \pm 11^{\text {ch }}$ & $203 \pm 9$ & anis \\
\hline PHE & $74 \pm 4$ & $95 \pm 10$ & $123 \pm 7$ & $189 \pm 16^{*}$ & $84 \pm 6$ & $126 \pm 14^{\text {th }}$ & $159 \pm 10$ & $m$ \\
\hline RP & $23 \pm 2$ & $22 \pm 3$ & $33 \pm 2$ & $53 \pm \quad 6$ & $24 \pm 1$ & $41 \pm 4^{a}$ & $52 \pm 2$ & mis \\
\hline BCAA & $329 \pm 7$ & $343 \pm 8$ & $332 \pm 11$ & $338 \pm 29$ & $317 \pm 15$ & $312 \pm 16$ & $319 \pm 11$ & \\
\hline$A A$ & $162 \pm 9$ & $175 \pm 11$ & $264 \pm 12$ & $392 \pm 24$ & $168 \pm 8$ & $267 \pm 23^{\text {का }}$ & $362 \pm 16$ & chis \\
\hline AN & $2270 \pm 250$ & $32106 \pm 664$ & $34427 \pm 576$ & - $35986+1023^{\circ}$ & $31352 \pm 440$ & $31961 \pm 532$ & $33869 \pm 828$ & \\
\hline
\end{tabular}

Data are expressed as mean $\pm S E M$ in $\mu$ mole kg wet weight ${ }^{1}(w w)$. For significance symbols, see Table 1. GABA: $\gamma$ aminobutyric acid.

Glutamine (Figure 3; Table 1, 3\&4) Arterial glutamine concentrations (Figure 3, upper panel) were significantly higher in PCSBDL compared with all other groups, but no major differences were observed between the PCS-PF, PF and NORM group. Cerebral cortex glutamine flux (Figure 3, middle panel) did not significantly differ from zero in the NORM and $\mathrm{PF}$ group. However, in both hyperammonemic groups glutamine was released in 
comparable amounts from the cerebral cortex into the blood. Cerebral cortex tissue glutamine concentrations (Figure 3, lower panel) were similar in NORM and PF rats, and were elevated in both chronic liver insufficiency models compared with NORM and PF rats. The elevation of tissue glutamine levels was more pronounced in the PCSBDL group at day 14.

Glutamate (Figure 4; Table 1, 3\&4) Arterial glutamate levels (Figure 4, upper panel) were comparable in PF, PCS-PF and PCSBDL rats, and decreased at day 14. Cerebral cortex glutamate fluxes (Figure 4, middle panel) did not significantly differ from zero in any of the groups. Cerebral cortex tissue glutamate levels (Figure 4, lower panel) were decreased in the PF group compared with NORM rats, but no differences were observed in tissue glutamate levels between PF, PCS-PF and PCSBDL rats.

Other amino acids (Table 1, 3\&4) Arterial BCAA levels were decreased in the PCSBDL group at both time points and in PCS-PF rats at day 14. Cerebral cortex BCAA fluxes did not differ from zero in most groups, and no BCAA uptake by the cerebral cortex was observed. Cerebral cortex tissue BCAA levels were similar in all groups. The arterial levels of the aromatic amino acids (AAA) phenylalanine and tyrosine increased in PCS-PF compared with PF, and in PCSBDL compared with PCS-PF rats. Interestingly, in both hyperammonemic groups a similar release of AAA from the cerebral cortex was observed, whereas cerebral cortex AAA flux did not differ from zero in the PF and NORM group. Cerebral cortex tissue AAA concentrations were elevated in PCSBDL rats compared with both PCS-PF and PF rats, and in PCS-PF compared with $\mathrm{PF}$ rats. A similar pattern was observed for tissue tryptophan levels.

Glucose and lactate (Table 1\&3) Arterial glucose concentrations were decreased in PCSBDL and PCS-PF compared with PF and NORM rats. Lactate concentrations were elevated in PCSBDL compared with all other groups. Glucose was taken up by the cerebral cortex in comparable amounts in all groups. Lactate fluxes were in general not significantly different from zero.

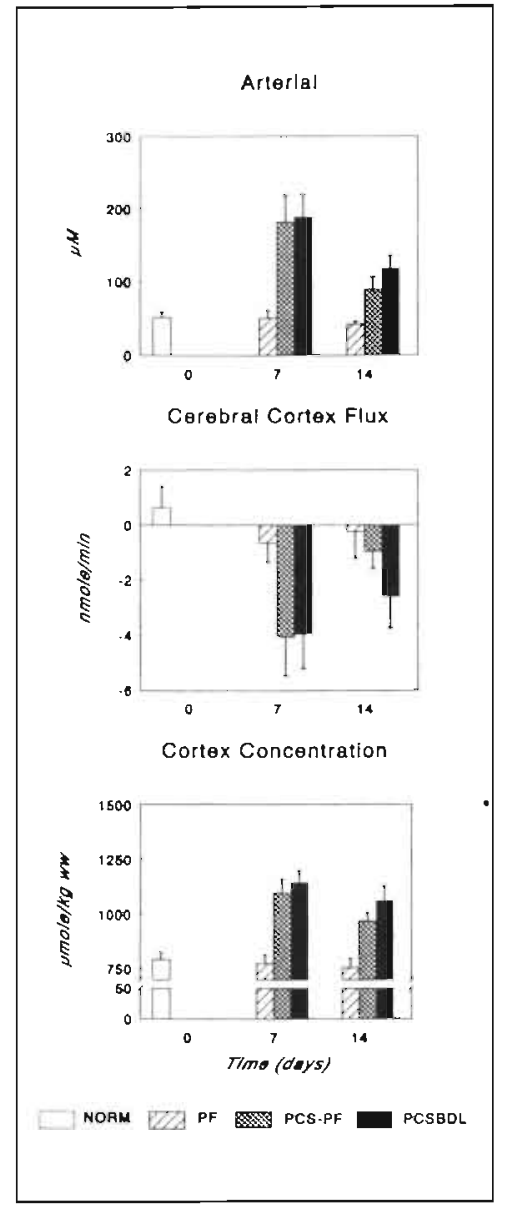

Figure 2. Ammonia: arterial concentrations (upper panel; $\mu \mathrm{M}$ ), cerebral cortex fluxes (middle panel; nmole $\min ^{-1}$ ) and cerebral cortex tissue concentrations (lower panel; umole kg wet $w^{-1}$ ) in normal control rats (NORM, open bars), sham operated pair-fed rats (PF, hatched bars), portacaval shunted pair-fed rats (PCS-PF, cross hatched bars) and in rats with portacaval shunts and bile duct ligation (PCSBDL, filled bars). Data are means $\pm S E M ; N=7$ or 8 per group. 


\section{DISCUSSION}

In the present experiments we investigated cerebral cortex ammonia and glutamine exchange, as well as the potential effects of this metabolite exchange across the cerebral cortex on cerebral cortical metabolism and function in two models of chronic liver insufficiencyinduced hyperammonemia during standardized food intake. Studies were performed 1 and 2 weeks after surgery, because we previously showed that buttonportacaval-shunted rats recover from several of the metabolic disturbances of portacaval shunting after 3-4 weeks (e.g. reflected by decreasing plasma ammonia levels) (26), which has been attributed to the formation of hepatopetal shunts $(26,27)$. Absence of differences in food intake and body weight in the PCSBDL, PCS-PF and $\mathrm{PF}$ group suggests that the differences between PCS-PF and PF rats are mainly due to the effects of portacaval shunting. Similarly, the differences between the PCSBDL group and PCS-PF rats are related to the sequelae of aggravated parenchymal liver injury and cholestatic jaundice, induced by common bile duct ligation $(28,29)$. Although the pair-feeding warranted quantitatively similar food intakes in all rats and can be used to exclude the main effects of differences in food intake, it does not circumvent the problem that the less anorectic PCS-PF and PF rats will consume their ration faster than the PCSBDL rats and therefore will remain starved for a longer period of the day.

In both chronic liver insufficiency groups the cerebral cortex showed net ammonia uptake, providing evidence that the rat cerebral cortex contributes to the metabolic adaptation of the organism to chronic hyperammonemia by net removal of ammonia from the body pools. The elevated tissue ammonia levels in both hyperammonemic groups, closely resembling those reported by Swain et al. (30), demonstrate that ammonia transformation to glutamine by the GS reaction, probably the main route of ammonia detoxification in the cerebral cortex in vivo, is insufficient to counteract the continuous net ammonia uptake.

Unidirectional whole brain ammonia uptake has repeatedly been confirmed (for review, see (9)), but to our knowledge only one report has been published on net ammonia uptake by the rat brain. In that study by Gjedde and colleagues, net ammonia uptake by the brain in 8 weeks portacaval shunted rats was observed, but not in 4 weeks shunted rats (8). In
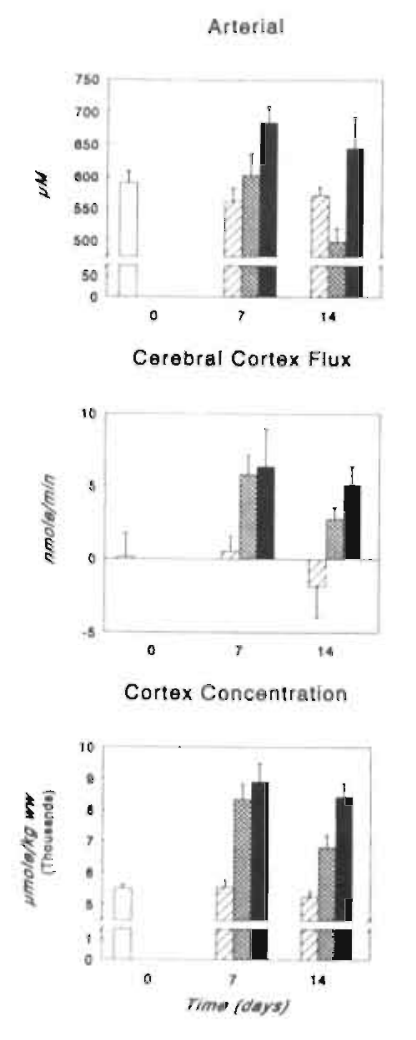

HOPM

Figure 3. Glutamine: anterial concentrations (upper panel: $\mu \mathrm{M}$ ), cerebral cortex fluxes (middle panel; nmolemin') and cerebral cortex tissue conceritrations (lower panel; umole $\mathrm{kg}$ wet $\mathrm{wt}^{-1}$ ) in normal control rats (NORM, open bars), sham operated pair-ted rats ( $P F$, hatched bars), portacaval shunted pair-fed rats (PCS-PF, cross hatched bars) and in rats with portacaval shunts and bile duct ligation (PCSBDL, filled bars). Data are means $\pm S E M ; N=7$ or 8 per group. 
normal humans, Lockwood and colleagues calculated the unidirectional ammonia uptake to be approximately $47 \%$, whereas net ammonia extraction was only $11 \%$ (1). In normal rats, a net extraction of $11 \%$ would mean an arteriovenous difference across the brain of no more than $5 \mu \mathrm{M}$. Given the coefficient of variance of the enzymatic ammonia determination (4\%; this study) it is clear that such a net venous-arterial concentration difference is extremely difficult to measure, explaining why we and others have been unable to detect net ammonia uptake by the normal rat brain (9).

Concerning the mechanism of the enhanced ammonia uptake in our experiments in the chronic liver insufficiency groups, Lockwood and colleagues have recently suggested an interesting mechanism (14). Using positron emission tomography they demonstrated that the observed enhanced unidirectional ammonia uptake in the brain of cirrhotic patients might be due to an enhanced permeability surface area product for ammonia (14). This could explain increased sensitivity to toxins as liver disease progresses, and ammonia-induced hepatic encephalopathy at normal or near normal ammonia levels (14).

Ammonia is an important participant in several reactions in the brain, among which the GS reaction, primarily localized in astrocytes (31). Glutamine produced in astrocytes is made available to neurons, providing substrate for neurotransmitter glutamate and GABA. Neuronally released glutamate and GABA, not recaptured by the neuron, can be taken up and recycled to glutamine by the astrocyte: the 'glutamine cycle' $(9,15,32,33)$. Despite its physiological importance in these and related reactions, ammonia is directly neurotoxic at elevated levels $((10-12)$; for reviews, see: $(3,5,9))$. For this reason, the astrocytic GS reaction, catalyzing the synthesis of the non-toxic amino acid glutamine from glutamate and ammonia, has been viewed as an important ammonia detoxification pathway within the brain $(15,33)$, but also in interorgan ammonia transport (1).

Indeed, ammonia delivered to the brain is rapidly incorporated into glutamine by the GS reaction $(1,13,34)$. Ongoing glutamine synthesis during hyperammonemia has therefore been suggested to explain increased brain glutamine levels in humans and experimental animals $(2,5,35)$. Elevated brain glutamine levels have been implicated in the pathogenesis of brain edema formation during acute hyperammonemia $(30,36)$, which might contribute to the pathogenesis of hepatic encephalopathy. 
Whether elevated brain glutamine levels always lead to net glutamine efflux from the brain has been a matter of considerable debate (reviewed in (9)). The present data show, in a situation of elevated cerebral cortex glutamine levels, net cerebral cortex glutamine release in both hyperammonemic groups, suggesting that the rat cerebral cortex contributes to ammonia detoxification not only by ammonia trapping and augmenting the tissue glutamine pools, but also by releasing glutamine. Because nutritional status, as assessed by food intake and body weight, was similar in the chronic liver insufficiency and PF rats, it must be concluded that the observed glutamine release from the cerebral cortex during hyperammonemia is mainly due to liver insufficiency and resulting hyperammonemia. This glutamine release from the cerebral cortex could provide a means of transporting ammonia in a nontoxic form to the kidney (or diseased liver), thereby constituting a means of definitive ammonia disposal.

The small amounts of glutamine released from the cerebral cortex in our studies suggest that this ammonia detoxification pathway is quantitatively not very important compared to other organs. However, according to the original 'glutamate depletion theory' the continuous glutamine efflux in both hyperammonemic groups could put a drain on the cerebral cortex total glutamate pool (9). It should be noted that a continuous drain of $4 \mathrm{nmole} / \mathrm{min}$ during 1 day would be sufficient to consume all the glutamate in the cerebral cortex in the absence of new glutamate formation (assuming cerebral cortex to weigh approximately 0.5 gram). This drain on cerebral cortex glutamate pools might be counter-balanced by cerebral cortex glutamate uptake, or altematively BCAA uptake and subsequent transamination $(37,38)$. However, neither glutamate nor BCAA uptake was observed in any group. The absence of any glutamate uptake is probably attributable to the relative impermeability of the blood-brain barrier for glutamate $(9,39)$. These negative observations raise the question of the source of the carbon skeletons of glutamate for glutamine synthesis. It is attractive to assume that the observed glucose uptake furnishes 2-oxoglutarate in the chronic liver insufficiency groups via the pyruvate carboxylase pathway $\left(\mathrm{CO}_{2}\right.$-fixation), for which the enzymatic machinery is present in brain mainly in the astrocytes $(15,32)$.

Another pathway for glutamate supply might be from protein degradation. In both liver insufficiency-induced hyperammonemia groups, enhanced net efflux of the aromatic amino acids was observed. This could imply that in the chronic liver insufficiency groups the cerebral cortex is in a net protein catabolic state, which seems to be supported by the observation that cerebral protein synthesis is inhibited after portacaval shunting (40). Such an increased net protein breakdown could provide precursors for glutamine synthesis and this could contribute to the observed glutamine release.

The changes in ammonia and glutamine exchange across the cerebral cortex make it of special interest to consider the consequences for the total cerebral cortex tissue pools of some transmitter amino acids, although these data do not necessarily reflect alterations in neurotransmission or compartmental changes $(32,41,42)$. Cerebral cortex glutamate concentrations were decreased in the PF group compared to normal unoperated control rats, but no differences in cerebral cortex tissue glutamate concentrations were observed between the PF group and both hyperammonemic groups. It must be emphasized that, despite considerable differences in plasma and tissue ammonia and glutamine, as well as net metabolite exchange across the cerebral cortex, total cerebral cortex glutamate pools apparently remained unaffected. This suggests that in the present experiments food intake, 
nutritional status or surgical trauma are probably more important in determining total tissue glutamate pools than hyperammonemia or liver insufficiency per se. Also, rats from the PCSBDL groups appeared to be more encephalopathic than PCS-PF rats, despite similar tissue glutamate and ammonia levels. The fact that tissue glutamine levels were higher in PCSBDL rats than in PCS-PF could suggest that ammonia metabolism rather than ammonia toxicity is the culprit of hepatic encephalopathy (43-46). Evidence pointing in that direction has also been provided in the past by Hourani and colleagues, demonstrating a close correlation between cerebrospinal fluid glutamine levels and encephalopathy stage in cirrhotics (47), and by Hawkins and colleagues who observed increased brain glutamine and unchanged glutamate levels after acute ammonia injection in rats (48). Another explanation would reside in reciproque changes in glutamate concentrations in different compartments (49). Thus, research on the role of glutamate (depletion) in the pathogenesis of hepatic encephalopathy probably should be directed to the releasable neurotransmitter glutamate pool $(42,49,50)$ and should take nutritional status into account.

Cerebral cortex GABA concentrations were similar in all groups. Although increased brain GABA levels have been reported in models of fulminant hepatic failure $(17,24,30)$, brain GABA levels generally are reported to be normal in chronic liver insufficiency models as well as in humans with chronic liver disease $((51)$; for reviews, see $(4,52))$. This has been interpreted as evidence against an important role for GABA in the pathogenesis of hepatic encephalopathy $(21,51,53)$. Our data seem to be compatible with this view. Also, it has been suggested that a substantial portion of the glutamine pool is linked to GABA synthesis $(33,41,54)$. This has been proposed to explain elevated brain GABA levels during hyperammonemia accompanied by increased brain glutamine concentrations as a metabolic rather than a neurotransmitter event $(17,30)$. Such a glutamine-related GABA increase was not confirmed in the present experiments, since cerebral cortex GABA levels remained unchanged despite increased tissue glutamine concentrations. In view of the compartmentation of GABA metabolism $(32,54)$ and GABA-synthesizing enzymes $(55,56)$ a definitive conclusion concerning the mechanisms underlying the present observations does not seem justified. It is attractive, however, to postulate a role for the 'GABA shunt' pathway (57) to explain the differences between the present data and those recently reported during acute hepatic failure $(17,30)$. Because any degree of hyperammonemia accelerates glycolysis by phosphofructokinase disinhibition $(5,9)$ and severe hyperammonemia in addition inhibits the TCA cycle at the level of 2-oxoglutarate dehydrogenase (9), increased flux through the 'GABA shunt' (glutamate to GABA, GABA to succinic acid semi-aldehyde, and ultimately to succinate) could explain increased GABA levels during acute severe hyperammonemia.

The present study shows that during chronic liver insufficiency-induced hyperammonemia, the cerebral cortex of the rat contributes to net ammonia detoxification by augmenting tissue ammonia and glutamine pools, and by net glutamine release into the blood. Under conditions of food intake standardization by pair-feeding techniques in rats with 1-2 weeks of liver insufficiency-induced hyperammonemia and appropriate controls, cerebral cortex ammonia uptake, glutamine accumulation by enhanced synthesis and/or breakdown inhibition, and subsequent glutamine release into the blood, appeared to be unrelated to the observed decreased cortical glutamate and unchanged GABA levels. These findings suggest that nutritional status and/or surgical trauma may play a crucial role in determining total cerebral cortex tissue concentrations of important metabolites. The exact importance of these findings for the field of hepatic encephalopathy research remains to be determined, 
especially because it is uncertain whether the results can be extrapolated to acute hyperammonemia studies. In these models, semi-starvation plays a less important role and contradictory results have been reported concerning tissue glutamate levels $(11,48,58,59)$. However, it seems that the 'glutamate depletion theory' in its most primitive form cannot explain the present results. The more pronounced degree of hepatic encephalopathy in the portacaval shunt/biliary obstruction group, despite similar arterial ammonia levels and total cerebral cortex tissue ammonia and glutamate levels suggest that other factors play a role in the pathogenesis of hepatic encephalopathy. Probably, part of this problem could be solved by directing research on the role of glutamatergic and/or GABA-ergic neurotransmission towards the releasable neurotransmitter pools. Finally, we conclude that the portacaval shunted/bile duct ligated rat provides a suitable model for studying the metabolic consequences of chronic liver insufficiency-induced hyperammonemia.

\section{REFERENCES}

1. Lockwood AH, McDonald JM, Reiman RE, Gelbard AS, Laughlin JS, Duffy TE, Plum F. The dynamics of ammonia metabolism in man. Effects of liver disease and hyperammonemia. J Clin Invest 1979:63:449-460.

2. Zieve L. Pathogenesis of hepatic encephalopathy. Metab Brain Dis 1987;2:147-165.

3. Butterworth RF. Pathophysiology of hepatic encephalopathy; the ammonia hypothesis revisited. In: Progress in Hepatic Encephalopathy and Metabolic Nitrogen Exchange (Bengtson F, Almdal T. Jeppsson B, Vilstrup H, eds.), Par 1, pp. 9-24. CRC Press, Boca Raton, 1991.

4. Record CO. Neurochemistry of hepatic encephalopathy. Gut 1991;32:1261-1263.

5. Duffy TE, Plum F. Hepatic Encephalopathy, in The liver: Biology and pathobiology (Arias I, Popper H, Schachter D, Shafritz DA, eds.), pp. 693-715. Raven Press, New York, 1982.

6. Sherlock S. Diseases of the liver and biliary system. Blackwell Scientific Publications, Ox ford, 1989.

7. Meijer AJ, Lamers WH, Chamuleau RAFM. Nitrogen metabolism and omithine cycle function. Physiol Rev 1990;70:701-748.

8. Gjedde A, Lockwood AH, Duffy TE, Plum F. Cerebral blood flow and metabolism in chronically hyperammonemic rats: Effect of an acute ammonia challenge. Ann Neurol 1978;3:325-330.

9. Cooper AJL, Plum F. Biochemistry and physiology of brain ammonia. Physiol Rev 1987;67:440-519.

10. McKhann GM, Tower DB. Ammonia toxicity and cerebral oxidative metabolism. Am J Physiol 1961;200:420-424.

11. Lin S, Raabe W. Ammonia intoxication: effects on cerebral cortex and spinal cord. J Neurochem 1985;44:1252-1258.

12. Lai JCK, Cooper AJL. Neurotoxicity of ammonia and fatty acids: differential inhibition of mitochondrial dehydrogenases by ammonia and fatty acyl coenzyme A derivatives. Neurochem Res 1991; 16:795-803.

13. Cooper AJL, Mora SN, Cruz NF, Gelbard AS. Cerebral ammonia metabolism in hyperammonemic rats. J Neurochem 1985;44:1716-1723.

14. Lockwood AH, Yap EWH, Wong W-H. Cerebral ammonia metabolism in patients with severe liver disease and minimal hepatic encephalopathy. J Cereb Blood Flow Metab 1991;1 1:337-441.

15. Shank RP, Aprison MH. Present status and significance of the glutamine cycle in neural tissue. Life Sciences 1981;28:837-842.

16. Dejong CHC, Deutz NEP, Soeters PB. Inter-organ nitrogen exchange during prolonged starvation in the rat. J Clin Nutr Gastroenterol 1991;6:176-183.

17. Dejong CHC, Kampman MT, Deutz NEP, Soeters PB. Cerebral cortex ammonia and glutamine metabolism during liver insufficiency-induced hyperammonemia in the rat. $\mathrm{J}$ Neurochem 1992;59:1071-1079.

18. Butterworth RF, Girard G, Giguère JF. Regional differences in the capacity for ammonia removal by brain following portacaval anastomosis. J Neurochem 1988;51:486-490.

19. Mullen KD, McCullough AJ. Problems with animal models of chronic liver disease: Suggestions for improvement in standardization. Hepatology 1989;9:500-505. 
20. Ribeiro J, Nordlinger B, Ballet F, Cynober L, Coudray-Lucas C, Baudrimont M, Legendre C, Delelo R, Panis Y. Intrasplenic hepatocellular transplantation corrects hepatic encephalopathy in portacaval shunted rats. Hepatology 1992;15: 12-18.

21. Maddison JE, Dodd PR, Morrison M, Johnston GAR, Farrell GC. Plasma GABA, GABA-like activity and the brain GABA-benzodiazepine receptor complex in rats with chronic hepatic encephalopathy. Hepatology 1987;7:621-628.

22. Dejong CHC, Kampman MT, Deutz NEP, Soeters PB. Altered glutamine metabolism in rat portal drained viscera and hindquarter during hyperammonemia. Gastroenterology 1992; 102:936-948.

23. Dejong CHC. Deutz NEP, Sceters PB. A simple new method for repeated in vivo cerebral cortex flux measurement in rats. Lab Anim Sci 1992;42:280-285.

24. Mans AM, Saunders SJ, Kirsch RE, Biebuick JF. Correlation of plasma and brain amino acid and putative neurotransmitter alterations during acute hepatic coma in the rat. J Neurochem 1979;32:285292.

25. van Eijk HMH, van der Heijden MAH, van Berlo CLH, Soeters PB. Fully automated liquidchromatographic determination of amino acids. Clin Chem 1988;34:2510-2513.

26. de Boer JEG, Oostenbroek RJ, van Dongen JJ, Janssen MA, Soeters PB. Sequential metabolic characteristics following portacaval shunts in rats. Eur Surg Res 1986; 18:96-106.

27. Kyu MH, Cavanagh JB. Some effects of portocaval anastomosis in the male rat. Br J Exp Pathol 1970;51:217-227.

28. Kountoumas J, Billing BH, Scheuer PJ. Prolonged bile duct obstruction: a new experimental modeI for cirrhosis in the rat. Br J Exp Pathol 1984;65:305-311.

29. Greve JW, Gouma DJ, Soeters PB, Buurman WA. Suppression of cellular immunity in obstructive jaundice is caused by endotoxins. A study in germ-free rats. Gastroenterology 1990;98:478-485.

30. Swain M, Butterworth RF, Blei AT. Ammonia and related amino acids in the pathogenesis of brain edema in acute ischemic liver failure in rats. Hepatology 1992;15:449-453.

31. Martinez-Hemandez A. Bell KP, Norenberg MD. Glutamine synthetase: glial localization in brain. Science 1977; 195:1356-1358.

32. Brainard JR, Kyner E, Rosenberg GA. ${ }^{13} \mathrm{C}$ nuclear magnetic resonance evidence for $\gamma$-aminobutyric acid formation via pyruvate carboxylase in rat brain: a metabolic basis for compartmentation. $J$ Neurochem 1989;53:1285-1292.

33. Waniewski RA. Physiological levels of ammonia regulate glutamine synthesis from extracellular glutamate in astrocyte cultures. J Neurochem 1992;58:167-174.

34. Cooper AJL, McDonald JM, Gelbard AS, Gledhill RF. Duffy TE. The metabolic fate of ${ }^{13} \mathrm{~N}$-labeled ammonia in rat brain. J Biol Chem 1979;254:4982-4992.

35. Bosman DK, Deutz NEP, de Graaf AA, van der Hulst RWN, van Eijk HMH, Bovée WMMJ, Maas MAW, Jöming GGA, Chamuleau RAFM. Changes in brain metabolism during hyperammonemia and acute liver failure: Results of a comparative 'H-NMR spectroscopy and biochemical investigation. Hepatology 1990;12:281-290.

36. Takahashi H, Koehler RC, Brusilow SW, Traystman RJ. Inhibition of brain glutamine accumulation prevents cerebral edema in hyperammonemic rats. Am J Physiol 1991;261:H825-H829.

37. Watanabe A, Shiota T, Takei N, Fujiwara M, Nagashima H. Ammonia detoxification by accelerated oxidation of branched chain amino acids in brains of acute hepatic failure rats. Biochem Med Metab Biol 1986; 35:367-375

38. Murthy CRK, Hertz L. Comparison between acute and chronic effects of ammonia on branched-chain amino acid oxidation and incorporation into protein in primary cultures of astrocytes and of neurons. J Neurosci Res 1987; 17:271-276.

39. Oldendorf WH, Szabo J. Amino acid assignment to one of three blood-brain amino acid carriers. Am J Physiol 1976;230:94-98.

40. Wasterlain CG, Lockwood AH, Conn M. Chronic inhibition of brain protein synthesis after portacaval shunting. Neurology 1978;28:233-238.

41. Fonnum F, Paulsen RP. Comparison of transmitter amino acid levels in rat globus pallidus and neostriatum during hypoglycemia or after treatment with methionine sulfoximine or $\gamma$-vinyl $\gamma-$ aminobutyric acid. J Neurochem 1990;54:1253-1257.

42. Maddison JE, Watson WEJ, Dodd PR, Johnston GAR. Alterations in cortical $\left[{ }^{3} \mathrm{H}\right]-$ kainate and $\alpha-\left[{ }^{3} \mathrm{H}\right]-$ amino-3-hydroxy-5-methyl-4-isoxazolepropionic acid binding in a spontaneous canine model of chronic hepatic encephalopathy. J Neurochem 1991;56:1881-1888.

43. Warren KS, Schenker S. Effect of an inhibitor of glutamine synthesis (methionine sulfoximine) on ammonia toxicity and metabolism. J Lab Clin Invest 1964;64:442-449. 
44. Jessy J, Mans AM, DeJoseph MR, Hawkins RA. Hyperammonemia causes many of the changes found after portacaval shunting. Biochem J 1990;272:311-317.

45. Hawkins RA, Jessy $\}$. Hyperammonemia does not impair brain function in the absence of net glutamine synthesis. Biochem J 1991;277:697-703.

46. Jessy J, DeJoseph MR, Hawkins RA. Hyperammonemia depresses glucose consumption throughout the brain. Biochem J 1991;277:693-696.

47. Hourani BT, Hamlin EM, Reynolds TB. Cerebrospinal fluid glutamine as a measure of hepatic encephalopathy. Arch Intern Med 1971;127:1033-1036.

48. Hawkins RA, Miller AL, Nielsen RC, Veech RL. The acute action of ammonia on rat brain metabolism in vivo. Biochem J 1973;134:1001-1008.

49. Bosman DK, Deutz NEP, Maas MAW, van Eijk HMH, Smit JJH, de Haan JG, Chamuleau RAFM. Amino acid release from cerebral cortex in experimental acute liver failure, studied by in vivo cerebral cortex micro dialysis. J Neurochem 1992;59:591-599.

50. Butterworth RF, Le O, Lavoie J, Szerb JC. Effect of portacaval anastomosis on electrically stimulated release of glutamate from rat hippocampal slices. J Neurochem 1991;56:1481-1484.

51. Roy S, Pomier-Layrargues G, Butterworth RF. Huet P-M. Hepatic encephalopathy in cirrhotic and portacaval shunted dogs: lack of changes in brain GABA uptake, brain GABA levels, brain glutamic acid decarboxylase activity and brain postsynaptic GABA receptors. Hepatology 1988;8:845-849.

52. Basile AS, Jones EA, Skolnick P. The pathogenesis and treatment of hepatic encephalopathy: evidence for the involvement of benzodiazepine receptor ligands. Pharmacol Rev 1991;43:27-71.

53. Mans AM, Kukulka KM, McAvoy KJ, Rokosz NC. Regional distribution and kinetics of three sites on the $\mathrm{GABA}_{\mathrm{A}}$ receptor: lack of effect of portacaval shunting. J Cereb Blood Flow Metab 1992; 12:334-346.

54. Hassel B, Paulsen RE, Johnson A, Fonnum F. Selective inhibition of glial metabolism in vivo by fluorocitrate. Brain Res 1992;576:120-124.

55. Kaufman DL, Houser CR, Tobin AJ. Two forms of the $\gamma$-aminobutyric acid synthetic enzyme glutamate decarboxylase have distinct intraneuronal distributions and cofactor interactions. J Neurochem 1991;56:720-723.

56. Rimvall K, Martin DL. Increased intracellular $\gamma$-aminobutyric acid selectively lowers the level of the larger of two glutamate decarboxylase proteins in cultured GABA-ergic neurons from rat cerebral cortex. J Neurochem 1992;58:158-166.

57. McGeer PL, McGeer EG. Amino acid neurotransmitters. In: Basic Neurochemistry (Siegel GJ, Albers RW, Agranoff BW, Katzman R, eds.), pp. 233-254. Little, Brown and Company, Boston, 1981.

58. Hindfeldt B, Siesjö BK. Cerebral effects of ammonia intoxication. Scand J Clin Lab Invest 1971;28:365-374.

59. O'Connor JE, Guerri C, Jordá A, Grisolía S. On the mechanism of the protective effect of ethanol against ammonia toxicity in mice. Biochem Biophys Res Comm 1982;107:1508-1516. 



\author{
Chapter 11
}

\title{
Kidney during chronic liver failure ${ }^{1}$
}

Submitted as: C.H.C. Dejong, N.E.P. Deutz, P.B. Soeters. Metabolic adaptation of the kidney to hyperammonemia during chronic liver insufficiency in the rat.

\section{ABSTRACT}

The aim of this study was to evaluate the role of renal ammonia and glutamine metabolism in the metabolic adaptation to chronic liver insufficiency-induced hyperammonemia in the rat. To this purpose, urinary excretion, renal net exchange and tissue concentrations of ammonia and amino acids were measured in anesthetized, normal unoperated control rats, in sham operated, in portacaval shunted and in portacaval shunted plus bile duct ligated rats. Sham operated and portacaval shunted rats were pair-fed to portacaval shunt/biliary obstruction rats, to correct for anorexia in that group, and all operated rats were studied 7 and 14 days after surgery.

Arterial ammonia as well as glutamine levels were elevated in portacaval shunted and portacaval shunt/biliary obstruction groups at all time points. At days 7 and 14, total renal ammonia production decreased in both portacaval shunted and portacaval shunt/biliary obstruction rats, associated with a 50\% decreased net renal glutamine uptake and strongly diminished net ammonia release into the renal vein, most prominent in the portacaval shunt/biliary obstruction group. Urinary ammonia excretion was similar in portacaval shunted and sham operated rats, but was more than 200\% increased at days 7 and 14 in portacaval shunt/biliary obstruction rats. In this group, in contrast to portacaval shunted rats, the kidney appeared to be an organ of net ammonia disposal from the body. In separate experiments in unanesthetized, unrestrained rats similar changes in urinary ammonia excretion were observed without changes in arterial $\mathrm{pH}$, excluding an effect of anesthesia or $\mathrm{pH}$ on the obtained results. The present results indicate that the kidney plays an important role in the metabolic adaptation to hyperammonemia during chronic liver insufficiency in the rat.

\footnotetext{
'This chapter is an exact copy of the original submission and consequently does not present all data of the experiments. Additional data are available on request.
} 


\section{IN'TRODUCTION}

Chronic liver disease is usually accompanied by some degree of hepatic cirrhosis, diminished urea synthesis capacity (1) and intra- or extrahepatic portasystemic shunting $(2,3)$, as well as abnormalities in nitrogen metabolism $(3,4)$, varying degrees of malnutrition (3) and immune system dysfunction $(5,6)$, probably related to impaired bile excretion $(5,7)$. In addition, a characteristic feature of chronic liver disease is the occurrence of hepatic encephalopathy $(3,4)$.

Recent hypotheses consider ammonia to be an important factor in the pathogenesis of hepatic encephalopathy $(1,8)$, since elevated plasma ammonia levels, either intermittently or continuously, are almost always found during chronic liver disease. This hyperammonemia during chronic liver disease is mainly caused by reduced hepatic urea synthesis capacity and portasystemic shunting, impairing and bypassing the main route of ammonia detoxification $(1,2)$.

Ammonia, despite its physiological importance in acid-base regulation and nitrogen transport, exerts several toxic effects at elevated levels on the organism $(2,9)$, and has been related to the occurrence of anorexia (10). Therefore, during liver insufficiency-induced hyperammonemia alternative pathways of ammonia detoxification become important. Probably the most important of these pathways is the synthesis of the non-essential amino acid glutamine, catalyzed by the cytosolic enzyme glutamine synthetase (11).

Glutamine synthesis and the resulting enlargement of the plasma and tissue free glutamine pools only constitutes a temporary form of ammonia detoxification, because glutamine can not be removed as such from the body in significant amounts. True ammonia disposal for the body requires either hepatic synthesis and urinary excretion of urea and/or renal ammonia excretion in the urine. Glutamine is the most important precursor for renal ammonia excretion. Thus, theoretically, high arterial glutamine and ammonia concentrations could favor its renal uptake and subsequent renal ammonia excretion in the urine, and in the past some data have been reported supporting this theory (12-17). Thus, artificial hyperammonemia in healthy volunteers turned the kidney into an organ of net ammonia uptake from the blood (15). Also, in cirrhotic patients renal ammonia release into the circulation decreased at elevated ammonia concentrations (13). However, the role of the amino acid glutamine in these metabolic processes is unclear and very little literature exists concerning the relationship between renal ammonia and glutamine metabolism in various liver insufficiency-induced hyperammonemic states. Thus, despite its pivotal role in nitrogen disposal and ammonia- and glutamine-related acid-base regulation $(15,18)$, little is known about renal nitrogen metabolism during hyperammonemia and conflicting results have been reported concerning glutamine fluxes across the normal rat kidney (19-26).

Recent studies in our laboratory during short-term, mild and severe hyperammonemia induced by portacaval shunting $\left(\mathrm{PCS}^{2}\right.$ ) and acute liver ischemia, respectively, (Dejong et al., unpublished observations, 1992) revealed that in the rat the kidney plays an important role in the adaptation to mild PCS-induced hyperammonemia by diminishing ammonia release into the renal vein and enhancing urinary ammonia excretion. During acute liver ischemia-induced hyperammonemia, however, no additional enhancement of renal ammonia disposal was observed. These observations raised the question of the importance of the renal adaptive response to liver insufficiency-induced hyperammonemia in a more chronic situation. 
Although portacaval shunting is a well-known, reproducible model for chronic liver insufficiency-induced hyperammonemia and hepatic encephalopathy $(4,27)$, portacaval shunting combined with common bile duct ligation was recently suggested to be a more reliable, new model for chronic hyperammonemia, and to induce a more pronounced degree of hepatic encephalopathy than PCS alone (28). This model combines the effects of portasystemic shunting with the occurrence of (biliary) cirrhosis of the liver, which could lead to a more pronounced degree of liver failure and also simulate the impaired bile excretion in patients with chronic hepatic cirrhosis.

To evaluate the role of renal ammonia and glutamine metabolism in the metabolic adaptation to chronic liver insufficiency-induced hyperammonemia in the rat, we studied the exchange of ammonia, glutamine as well as several other amino acids across the kidney in two models of 7 and 14 days of liver insufficiency: portacaval shunting, and portacaval shunting plus bile duct ligation, as well as in sham operated and normal unoperated control rats. In addition, urinary ammonia and amino acid excretion and kidney tissue concentrations were measured. To reduce the effects of differences in food intake, all operated rats received equal amounts of food. Our results suggest that the kidney plays an important role in the metabolic adaptation to hyperammonemia during chronic liver insufficiency in the rat.

\section{MATERIALS AND METHODS}

Animals Male specified pathogen free Wistar rats $(N=54,300 \pm 25 \mathrm{~g}$, random bred Wistar/CPB:WU/Bor, Winkelmann, Borchen, F.R.G.) were used throughout and were housed under standard conditions ( $12 \mathrm{~h}$ light cycle: $8 \mathrm{AM}-8 \mathrm{PM}$ ) and received standard pellet lab chow (RMH, Woerden, The Netherlands) and water ad libitum until entering the experiments i.e. until surgery. Rats were maintained and humanely cared for in accordance with the recommendations of the Guide for the Care and Use of Laboratory Animals, as applied in our institute.

Groups The following four groups were studied: 1 PCSBDL group. Portacaval shunting (PCS) plus bile duct ligation (BDL) was performed in one surgical session $( \pm 20 \mathrm{~min}$ ). After resuscitation from the surgery, rats were placed in metabolic cages with free access to standard pellet lab chow. Daily food intake was recorded and this amount was administered to fixed PCS and PF mates (see below) to eliminate effects of differences in food intake; 2 $P C S-P F$ group. In these rats, PCS was performed 1 day later than portacaval shunting/biliary obstruction in mates. Rats were allowed to recover, placed in metabolic cages and administered the amount of food eaten by their PCSBDL mate (pair-feeding: PF); $\underline{3} P F$ group. These rats underwent laparotomy and manipulations as in PCS-PF rats, but without shunting (sham operation). Rats were allowed to recover, placed in metabolic cages and administered the amount of food eaten by the PCSBDL mate (pair-feeding: PF). In the PCSBDL, PCS-PF and PF group, sampling was performed 7 or 14 days after surgery (see below); 4 NORM group. Sampling without previous surgery; normal control rats. These rats received food ad libitum.

Before surgery, rats were randomly assigned to one of these groups. Care was taken that surgical procedures were of equal duration in PCS-PF and PF rats and their individually 
paired PCSBDL mate. Body weight was measured at the time intervals indicated in the figures. Water was administered ad libitum in all groups.

Behavior Before entering the experiments and before sampling, behavior of all rats was studied during a 5 min period. In this behavioral assessment, specific attention was directed towards appearance, presence of lethargy, spontaneous locomotor activity and exploratory behavior (standing upright etc.), presence of tiptoe gait, toeing out and arched back, reaction to tail pinching and presence of ataxia. Since the PCSBDL rats were jaundiced, this staging procedure could not be performed in a blinded fashion.

Surgery Operations and sampling procedures were carried out under ether anesthesia at constant core temperatures. Portacaval shunting was performed by the button technique (29). Bile duct ligation was performed in the same session as portacaval shunting by double ligation with silk 4-0 and 6-0 near the liver and by single ligation near the duodenum after which $1.0 \mathrm{~cm}$ of the bile duct between the ligatures was resected. In the PCS-PF and PF group the bile duct was manipulated, but not dissected, as this would lead to stenosis and obstruction.

Sampling Prior to blood sampling, all rats were ovemight fasted. During the sample procedure, rectal temperature was maintained at $37 \pm 1^{\circ} \mathrm{C}$. PE 50 catheters (Intramedic $^{\mathrm{R}}$ Polyethylene Tubing PE-50: $0.058 \mathrm{~cm}$ I.D., $0.096 \mathrm{~cm}$ O.D., Clay Adams, Parsippany, NJ, U.S.A.) were inserted into the left jugular vein and right carotid artery. A $25 \mathrm{G}$ needle bearing silastic catheter (Silastic Medical Grade Tubing $0.051 \mathrm{~cm} \mathrm{I.D.,} 0.094 \mathrm{~cm}$ O.D., Dow Coming Corporation, Medical Products, Midland, MI, U.S.A.) was introduced in the left renal vein and cemented in place with cyanoacrylate adhesive. A piece of PE 10 tubing $(0.30$ $\mathrm{mm}$ I.D., $0.58 \mathrm{~mm}$ O.D.) was inserted into the left ureter.

For renal plasma flow (RPF) determination, a $1 \mathrm{mM}$ PAH solution (isomolaric, $\mathrm{pH} 7.40$ : Sigma A 1422, St. Louis, MO, U.S.A.) was infused into the jugular vein at a rate of approximately $0.08 \mathrm{~mL} \mathrm{~min}^{-1}$, including a $30 \mathrm{~min}$ equilibration period to attain steady state arterial PAH concentrations $(21,29)$, after a $0.032 \mathrm{~mL} \cdot 100 \mathrm{~g}$ body $\mathrm{wt}^{-1}$ priming dose of a $50 \mathrm{mM}$ PAH solution. The resulting arterial PAH concentrations $(<40 \mu \mathrm{M})$ are not likely to influence renal ammoniagenesis (24). For urine collections on ice, the free end of the PE 10 tubing was connected airtight to a $30 \mathrm{~cm}$ PE 50 catheter, led through a beaker containing ice. Our urine data in control rats are comparable to studies in which no PAH was used $(24,25,30-32)$, providing evidence for the reliability of the urine sampling method.

At steady state arterial PAH concentrations ( $\pm 30 \mathrm{~min}$ ), a $15 \mathrm{~min}$ urine collection was done. Urine volume was quantified with a Hamilton syringe and mixed with a fixed volume of icecold distilled water, divided in portions for amino acid, ammonia and urea determinations and immediately immersed in liquid nitrogen. After urine collection, $1 \mathrm{~mL}$ of blood was withdrawn simultaneously from the renal vein and carotid artery and kept on ice in heparinized tubes (Micro-tubes LH/1.3, Sarstedt, Nümbrecht, F.R.G.) during further processing before storage at $-70^{\circ} \mathrm{C}$. Finally, a clamp was placed on the renal pedicle and the kidney was freeze-clamped in liquid nitrogen (29). Tissues were stored at $-70^{\circ} \mathrm{C}$ until further processing. Rats were killed by an overdose of the anesthetic.

Metabolic cage experiments In a separate series of experiments, urinary ammonia and urea excretion as well as arterial acid-base parameters were determined in conscious, unrestrained rats of the same age and sex as those described above. To that purpose 36 rats, otherwise treated as described above, were randomly assigned to either the PCSBDL, PCS- 
Table 1. Arterial concentrations.

\begin{tabular}{|c|c|c|c|c|c|c|c|c|c|c|}
\hline & DAY 0 & & DAY 7 & & & & & DAY 14 & & \\
\hline & NORM & $P F$ & PCS-PF & & PCSBDL & & $\overline{P F}$ & PCS-PF & $\overline{\text { PCSBDL }}$ & \\
\hline BILI & $1.3 \pm 0.1$ & $1.2 \pm 0.1$ & $2.4 \pm 0.4$ & $h$ & $119.9 \pm 7.6$ & in & $1.3 \pm 0.1$ & $2.6 \pm 0.3$ & $112.9 \pm 7.3$ & af \\
\hline GLC & $10.2 \pm 0.4$ & $9.6 \pm 0.5$ & $9.6 \pm 0.3$ & & $8.7 \pm 0.3$ & & $9.5 \pm 0.3$ & $8.9 \pm 0.5$ & $8.4 \pm 0.2$ & $\infty \infty$ \\
\hline LAC & $2.6 \pm 0.3$ & $6.2 \pm 0.8^{n}$ & $9.7 \pm 0.3$ & h & $9.7 \pm 0.4$ & $h$ & $4.4 \pm 0.9$ & $8.0 \pm 1.0^{\circ}$ & $7.3 \pm 1.1$ & $\infty$ \\
\hline AMM & $59 \pm 13$ & $86 \pm 14$ & $223 \pm 19$ & $\pi$ & $170 \pm 13$ & ii & $51 \pm 6^{\circ}$ & $177 \pm 13$ & $101 \pm 16^{\mathrm{dot}}$ & $\infty$ \\
\hline UREA & $9.0 \pm 0.6$ & $9.2 \pm 0.6$ & $8.1 \pm 0.2$ & & $7.9 \pm 0.4$ & & $8.7 \pm 0.6$ & $7.0 \pm 0.4$ & $8.5 \pm 1.0$ & \\
\hline GLU & $68 \pm 14$ & $73 \pm 17$ & $96 \pm 18$ & & $61 \pm 17$ & 1 & $43 \pm 5$ & $65 \pm 8$ & $42 \pm 7$ & $d$ \\
\hline SER & $136 \pm 7$ & $130 \pm 7$ & $152 \pm 14$ & & $144 \pm 11$ & & $141 \pm 7$ & $129 \pm 13$ & $134 \pm 24$ & \\
\hline GLN & $479 \pm 17$ & $540 \pm 21^{m}$ & $558 \pm 26$ & & $640 \pm 33$ & 0 & $524 \pm 15$ & $494 \pm 21$ & $534 \pm 60$ & \\
\hline GLY & $245 \pm 11$ & $236 \pm 8$ & $227 \pm 12$ & & $240 \pm 14$ & & $253 \pm 9$ & $238 \pm 10$ & $219 \pm 25$ & 8 \\
\hline CIT & $37 \pm 1$ & $37 \pm 2$ & $44 \pm 3$ & & $49 \pm 2$ & $n$ & $38 \pm 2$ & $43 \pm 2^{\circ}$ & $39 \pm 3$ & $\infty$ \\
\hline ARG & $68 \pm 4$ & $85 \pm 6^{m}$ & $92 \pm 7$ & & $92 \pm 15$ & & $85 \pm 4$ & $92 \pm 6$ & $92 \pm 13$ & \\
\hline TYR & $50 \pm 2$ & $53 \pm 3$ & $78 \pm 8$ & n & $104 \pm 4$ & y & $49 \pm 4$ & $60 \pm 5^{\infty p}$ & $91 \pm 13$ & thin \\
\hline PHE & $55 \pm 3$ & $61 \pm 3$ & $78 \pm 5$ & h & $91 \pm 5$ & i & $58 \pm 4$ & $63 \pm 5^{\oplus}$ & $77 \pm 8$ & $\infty$ \\
\hline BCAA & $237 \pm 13$ & $260 \pm 22$ & $257 \pm 20$ & & $301 \pm 25$ & & $266 \pm 22$ & $205 \pm 18^{\circ}$ & $283 \pm 63$ & \\
\hline AAA & $105 \pm 4$ & $114 \pm 5$ & $157 \pm 12$ & $\mathrm{n}$ & $195 \pm 9$ & ik & $107 \pm 7$ & $123 \pm 9$ & $169 \pm 21$ & $\mathrm{etri}$ \\
\hline$\alpha A N$ & $2260 \pm 59$ & $2514 \pm 132$ & $2739 \pm 155$ & & $3174 \pm 145$ & $\mathrm{nj}$ & $2370 \pm 82$ & $2305 \pm 102^{P}$ & $2763 \pm 322$ & $\infty$ \\
\hline
\end{tabular}

Data are expressed as means \pm SEM in $\mu \mathrm{M}$, except urea, glucose (GLC) and lactate (LAC) in mM. BILI: total bilirubin. BCAA: branched-chain amino acids LEU, ILE and VAL. AAA: aromatic amino acids TYR and PHE. $\alpha$ AN: the sum of the individual amino acids measured. Symbols: ANOVA for group effects; $t=7$ to $t=14$ days: vs. PF $p<0.05,{ }^{0} p<0.01,{ }^{c} p<0.001$; vs. PCS-PF ${ }^{\circ} p<0.05,{ }^{0} p<0.01,{ }^{\prime} p<0.001$. Mann-Whitney $U$ for differences between groups: vs. $P F{ }^{0} p<0.05,{ }^{n} p<0.01, p<0.001$; vs. PCS-PF

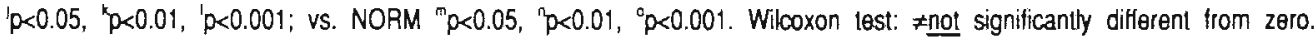
ONEWAY procedure for time effects within groups ${ }^{\circ} p<0.05$. Complete list of amino acids available on request.

PF or PF group ( $N=12$ per group) and placed individually in metabolic cages permitting urine collection in conscious unrestrained rats. After a 14 days pair-feeding period, these rats were ovemight fasted. In some of these rats ( $N=6$ per group) urine was collected on 2 $\mathrm{mL}$ of $6.0 \mathrm{~N} \mathrm{HCl}$ during the overnight fast to assess urinary ammonia and urea excretion and to measure urine production in conscious, unrestrained rats. In all rats, on the moming after the fasting period, between 8.00 and $10.00 \mathrm{AM}$, a PE 50 catheter was inserted under ether anesthesia into the right femoral artery, sealed and tunneled to the neck. Hereafter, rats were allowed to recover from surgery and placed individually in their cages. Approximately 1.5 hours after femoral artery catheter implantation, when rats were alert and freely moving in their cages, arterial blood was collected anaerobically in these unrestrained rats in heparinized syringes on ice for acid-base parameter determination on a blood gas analyzer (Acid Base Laboratory, ABL3, Radiometer, Copenhagen, Denmark) after correction for temperature.

Biochemical analysis Blood samples were kept on ice during further processing. Centrifugations were performed at $4^{\circ} \mathrm{C}$ during $5 \mathrm{~min}$ at $8,800 \mathrm{~g}$. Plasma was put into liquid nitrogen within $20 \mathrm{~min}$. All samples were stored at $-70^{\circ} \mathrm{C}$. Hematocrit was obtained with a microfuge. Plasma glucose, lactate, and plasma and urine ammonia and urea were determined enzymatically (29), and plasma and urine amino acids by HPLC technique as 
Table 2. Behavioral assessment.

\begin{tabular}{|c|c|c|c|c|c|c|c|c|c|c|}
\hline & \multirow{2}{*}{$\begin{array}{l}\text { DAYO } \\
\text { NORM }\end{array}$} & \multicolumn{4}{|c|}{ DAY 7} & \multicolumn{5}{|c|}{ DAY 14} \\
\hline & & $\overline{P F}$ & PCS-PF & PCSBD & & $\mathrm{PF}$ & PCS-PF & & PCSBDL & \\
\hline 'Gerbil' appearance & $0 / 8$ & $3 / 8$ & $4 / 8$ & $8 / 8$ & 9 & $4 / 8$ & $8 / 8$ & 9 & $6 / 6$ & \\
\hline Toeing out (hindlimbs) & $0 / 8$ & $2 / 8$ & $3 / 8$ & $8 / 8$ & $\pi$ & $3 / 8$ & $8 / 8$ & a & $6 / 6$ & a \\
\hline Tiptoe gait & $0 / 8$ & $0 / 8$ & $0 / 8$ & $8 / 8$ & st & $0 / 8$ & $0 / 8$ & & $6 / 6$ & $s$ \\
\hline 'Duck' gait & $0 / 8$ & $0 / 8$ & $0 / 8$ & $8 / 8$ & sv & $0 / 8$ & $0 / 8$ & & $5 / 6$ & n \\
\hline Arched back & $0 / 8$ & $0 / 8$ & $0 / 8$ & $8 / 8$ & si & $0 / 8$ & $2 / 8$ & & $6 / 6$ & w \\
\hline Ataxia & $0 / 8$ & $0 / 8$ & $1 / 8$ & $7 / 8$ & su & $1 / 8$ & $2 / 8$ & & $5 / 6$ & -9 \\
\hline
\end{tabular}

All items were scored on a dichotomic basis i.e. present or absent. $X N$ in the table indicates that the item was $X$ times present in $\mathrm{N}$ rats. Fischer's one-tailed exact test: vs. PF ${ }^{q} p<0.05,{ }^{\prime} p<0.01,{ }^{p} p<0.001$; vs. PCS-PF ${ }^{p} p<0.05,{ }^{4} p<0.01,{ }^{p} p<0.001$.

described previously (33). Tissue ammonia and amino acids were determined similarly in $5 \%$ sulfosalicylic acid (w/v; SSA) extracts (29). Water content was determined after freezedrying and calculated as described previously (34). Total bilirubin was determined in plasma with a commercially available test kit (Bilirubin-Test, Hoffmann-La Roche, Basle, Switzerland). The coefficient of variance for all determinations was well below $4 \%$ : coefficient of variance was $2 \%$ for glutamine, $4 \%$ for ammonia and $2 \%$ for urea and bilirubin $(29,33)$.

Calculations $\alpha$-Amino nitrogen was calculated by adding the concentrations of the individual amino acids measured $(29,33)$. Urea values were corrected for ammonia. Plasma $\mathrm{PAH}$ concentrations were calculated using the formula: $[\mathrm{PAH}]_{\text {plasma }}=[\mathrm{PAH}]_{\text {blood }}$. (100/(100-hematocrit)). RPF (mL min ${ }^{-1}$ ) was measured as described by Selkurt (35) and calculated using the standard equation $(19,29,36): R P F=I /\left([P A H]_{A}-[P A H]_{R}\right)$. In this equation, I is the amount of PAH infused $\left(\mu\right.$ mole $\left.\mathrm{min}^{-1}\right)$, and $[\mathrm{PAH}]_{\mathrm{R}(\mathrm{A})}$ is the concentration of PAH ( $\mu$ mole $\left.\cdot L^{-1}\right)$ in the renal venous $(R)$ and arterial (A) plasma respectively. This calculation is based on the assumption of steady state PAH concentrations, which in prior experiments (results not shown) was found to occur after $30 \mathrm{~min}$ of PAH infusion $(21,29)$. Fluxes were calculated as RPF times V-A difference and expressed per two kidneys (nmole $\cdot 100 \mathrm{~g}$ body $\mathrm{wt}^{-1} \mathrm{~min}^{-1}$ ). Positive figures mean net efflux, negative values reflect net uptake. Urinary excretion was calculated as urine production times urinary concentration and also expressed per two kidneys (nmole $\cdot 100 \mathrm{~g}$ body $\mathrm{wt}^{-1} \mathrm{~min}^{-1}$ )

Statistics (37) Data presented are means $\pm \mathrm{SEM}, \mathrm{P}<0.05$ was considered significant. Analysis of variance was used to test for group effects (ANOVA) and time effects within groups (ONEWAY). The Mann-Whitney $U$ non-parametric test was used for differences between groups at specific time points. Wilcoxon's nonparametric test was used for differences from zero. Fischer's exact test was used to test for differences in encephalopathy stages (one-tailed, binomial testing). Significances are indicated in Tables 1-6. 
Table 3. Renal fluxes and plasma flow.

\begin{tabular}{|c|c|c|c|c|c|c|c|c|c|}
\hline & DAY 0 & & DAY 7 & & & & DAY 14 & & \\
\hline & NORM & PF & PCS-PF & PCSBDL & & PF & PCS.PF & PCSBDL & \\
\hline RPF & $2.4 \pm 0.4$ & $2.0 \pm 0.2$ & $2.1 \pm 0.2$ & $2.8 \pm 0.3$ & 8 & $2.3 \pm 0.3$ & $2.4 \pm 0.3$ & $3.3 \pm 0.3$ & $\infty$ \\
\hline GLC & $-289 \pm 668 \neq$ & $605 \pm 510 \neq$ & $467 \pm 506 \neq$ & $371 \pm 493 \neq$ & $\neq$ & $1030 \pm 457$ & $810 \pm 513$ & \pm 538 & $\neq$ \\
\hline LAC & $-1001 \pm 147$ & $-1382 \pm 307$ & $-1753 \pm 301$ & $.1651 \pm 459$ & & $-1452 \pm 394$ & $-1568 \pm 367$ & $-1259 \pm 417$ & \\
\hline AMM & $181 \pm 44$ & $318 \pm 42^{m}$ & $176 \pm 25^{n}$ & $89 \pm 40$ & h & $248 \pm 57$ & $86 \pm 26^{\mathrm{cm}}$ & $11 \pm 29^{\text {cant }}$ & th \\
\hline UREA & $-1125 \pm 344$ & $-146 \pm 315^{m} \neq$ & $-300 \pm 315 \neq$ & $-413 \pm 185 \neq$ & $\neq$ & $-1006 \pm 719 \neq$ & $-459 \pm 261 \neq$ & $-1529 \pm 484$ & D \\
\hline GLU & $-11 \pm 6 \neq$ & $11 \pm 27^{n} \neq$ & $16 \pm 12 \neq$ & $56 \pm 19$ & & $23 \pm 8$ & $29 \pm 18 \neq$ & $26 \pm 20$ & $\neq$ \\
\hline SER & $242 \pm 18$ & $186 \pm 18^{m}$ & $126 \pm 18$ & $119 \pm 21$ & 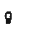 & $196 \pm 22$ & $116 \pm 16$ ch & $103 \pm 13$ & at \\
\hline GLN & $-309 \pm 35$ & $-368 \pm 35$ & $-177 \pm 41^{h}$ & $-139 \pm 18$ & 1 & $-336 \pm 28$ & $-107 \pm 20^{i}$ & $-158 \pm 30$ & on \\
\hline GLY & $-95 \pm 7$ & $-41 \pm 12^{\pi}$ & $-32 \pm 10$ & $-78 \pm 26$ & & $-88 \pm 17$ & $-45 \pm 18^{\circ}$ & $-58 \pm 16$ & \\
\hline CIT & $-49 \pm 3$ & $-42 \pm 2$ & $-48 \pm 5$ & $-59 \pm 3$ & & $-48 \pm 4$ & $-48 \pm 4$ & $-53 \pm 5$ & $\infty$ \\
\hline TAU & $-29 \pm 12$ & $0 \pm 10 \neq$ & $6 \pm 16 \neq$ & $-83 \pm 32^{a} \neq$ & & $15 \pm 16 \neq$ & $17 \pm 13 \neq$ & $-94 \pm 19$ & chis \\
\hline ARG & $35 \pm 12$ & $45 \pm 9$ & $67 \pm 12$ & $45 \pm 9$ & & $48 \pm 6$ & $51 \pm 5$ & $57 \pm 6$ & \\
\hline TYR & $17 \pm 2$ & $21 \pm 3$ & $18 \pm 3$ & $19 \pm 5$ & & $18 \pm 4$ & $18 \pm 2$ & $19 \pm 5$ & \\
\hline PHE & $-8 \pm 2$ & $-10 \pm 1$ & $-13 \pm 2$ & $-8 \pm 3$ & & $-12 \pm 4$ & $-9 \pm 6 \neq$ & $-7 \pm 5$ & $\neq$ \\
\hline BCAA & $32 \pm 6$ & $35 \pm 8$ & $21 \pm 6$ & $41 \pm 12$ & & $32 \pm 6$ & $18 \pm 18 \neq$ & $32 \pm 5$ & \\
\hline AAA & $9 \pm 1$ & $10 \pm 3$ & $4 \pm 5 \neq$ & $10 \pm 5$ & & $6 \pm 3 \neq$ & $9 \pm \quad 8 \neq$ & $12 \pm 4$ & \\
\hline$\alpha$ AN & $-144 \pm 61$ & $-108 \pm 104 \neq$ & $30 \pm 93 \neq$ & $-72 \pm 116 \neq$ & & $-124 \pm 45$ & $142 \pm 89^{40} \mp$ & $-180 \pm 117$ di & $a \neq$ \\
\hline
\end{tabular}

Data are means $\pm S E M$ in nmole $100 \mathrm{~g}$ body $\mathrm{wt}^{-1} \mathrm{~min}^{-1}$ per two kidneys, except plasma flows in $\mathrm{mL} \cdot 100 \mathrm{~g}$ body $\mathrm{wt}^{-1} \mathrm{~min}^{-1}$ per two kidneys. For significance symbols, see Table 1. Complete list of amino acids available on request.

\section{RESULTS}

General Food intake and changes in body weight were similar in PCSBDL, PCS-PF and PF rats (Figure 1). No signs of systemic or local inflammation or impaired wound healing were observed in the PCSBDL group. Arterial total bilirubin levels (Table 1) were slightly increased in PCS-PF compared to PF rats, as has been reported previously (27), and were strongly elevated in PCSBDL rats compared to all other groups.

Behavior (Table 2) PCSBDL rats had a characteristic ill-groomed 'gerbil' appearance with bristly hair. Also in these rats, subtle signs were observed pointing to more pronounced encephalopathy, such as decreased spontaneous locomotor activity and exploratory behavior, more abnormalities in gait and hindlimb ataxia.

Ammonia (Figure $2 \& 3$; Table 1, 3-5) Arterial ammonia levels were elevated in both PCS-PF and PCSBDL rats, but less pronouncedly in the latter. Ammonia release into the renal vein was increased in PF compared with NORM rats. It decreased in both hyperammonemic groups compared with the PF group, but more pronouncedly in the PCSBDL group. Urinary ammonia excretion was similar in PCS-PF and PF rats, but was significantly enhanced in the PCSBDL group compared with both PF and PCS-PF rats. Kidney tissue ammonia concentrations were similar in all groups at day 7 . At day 14 , however, kidney tissue ammonia was lower in PCSBDL rats.

In both chronic liver insufficiency groups compared to the PF group the kidney reduced total apparent renal ammoniagenesis (flux + urinary excretion; Figure 3, upper panel) 
Table 4. Kidney tissue concentrations and water content.

\begin{tabular}{|c|c|c|c|c|c|c|c|c|c|c|}
\hline & & & & 7 & & & $A Y$ & & & \\
\hline & DRM & PF & PCS. & & PCSBDL & $\mathrm{PF}$ & PCS & & PCSBDL & \\
\hline $\mathrm{H}_{2} \mathrm{O}$ & $74.8 \pm 0.3$ & $75.5 \pm 0.4$ & $76.4 \pm$ & 0.6 & $76.4 \pm 0.5$ & $76.8 \pm 0.7$ & $75.4 \pm$ & 0.6 & $78.3 \pm 0.6$ & \\
\hline$A M$ & $2959 \pm 158$ & $3496 \pm 356$ & $3774 \pm$ & 370 & $3697 \pm 252$ & $3170 \pm 283$ & $3839+$ & 307 & $2250 \pm 213$ & \\
\hline$S P$ & $1230 \pm 81$ & $986 \pm 96^{m}$ & $809 \pm$ & 92 & $679 \pm 60$ & n $\quad 1012 \pm 98$ & $851 \pm$ & 27 & $854 \pm 72$ & \\
\hline & $3175 \pm 292$ & $3066 \pm 156$ & $2796 \pm$ & 129 & $2706 \pm 203$ & $2958 \pm 166$ & $2869+$ & 157 & & \\
\hline ER & $796 \pm 167$ & $491 \pm 23^{m}$ & $410 \pm$ & 210 & $413 \pm 19$ & $503 \pm 23$ & $416 \pm$ & $22 \infty$ & $32 \pm 26$ & \\
\hline$N$ & $1065 \pm 86$ & $7 \pm 61$ & 10334 & 55 & $\pm 81^{\prime}$ & $35 \pm 49$ & $1043 \pm$ & $28^{a 9}$ & \pm 89 & \\
\hline & $2474 \pm 500$ & $1992 \pm 153$ & $1769 \pm$ & 146 & $1834 \pm 132$ & $2176 \pm 161$ & 2015t & 187 & $1846 \pm 121$ & \\
\hline & $40 \pm \quad 4$ & $36 \pm 7$ & $46 \pm$ & 4 & $46 \pm 3$ & $9 \pm 4$ & $44 \pm$ & 6 & $44 \pm 7$ & \\
\hline 1 & $13664 \pm 960$ & $3061 \pm 844$ & $2531 \pm$ & 889 & $15411 \pm 579$ & * $12533 \pm 523$ & $12692 \pm$ & 678 & $14646 \pm 1639$ & \\
\hline 80 & $109 \pm 7$ & $133 \pm 7 m$ & $127 \pm$ & 7 & $131 \pm 10$ & $110 \pm 3$ & $125+$ & 6 & $29 \pm \quad 9$ & \\
\hline & $73 \pm 4$ & $85 \pm 4^{m}$ & $98 \pm$ & 8 & $128 \pm 6$ & $77 \pm \quad 5$ & $88+$ & 4 & $123 \pm 12$ & \\
\hline HE & $66 \pm 4$ & $76 \pm 5$ & $74 \pm$ & 6 & $102 \pm 8$ & $62 \pm 5$ & $67 \pm$ & 3 & $92 \pm 6$ & 6 \\
\hline 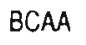 & $314 \pm 15$ & $330 \pm 13$ & $305 \pm$ & 16 & $355 \pm 23$ & $305 \pm 24$ & $265+$ & 14 & $315 \pm 42$ & \\
\hline 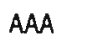 & $139 \pm 7$ & $161 \pm 8^{m}$ & 1724 & 12 & $229 \pm 14^{n}$ & $139 \pm 9$ & $155+$ & 6 & $215 \pm 17$ & \\
\hline AN & $4802 \pm 1696$ & $22919 \pm 645$ & $21717 \pm 1$ & 1140 & $24944 \pm 942$ & $22236 \pm 737$ & $22055+$ & 937 & $23974 \pm 1693$ & \\
\hline
\end{tabular}

Data are expressed as mean \pm SEM in $\mu$ mole $k g$ wet weight ${ }^{\prime}$ (ww). For significance symbols, see Table 1. Complete list of amino acids available on request.

$(\mathrm{P}<0.05)$. In addition, the fraction of total renal ammoniagenesis excreted in the urine (Figure 3, lower panel) was enhanced in PCSBDL versus both PF and PCS-PF rats $(\mathrm{P}<0.01)$.

Net renal ammonia disposal from, or addition to the body ammonia pools can be calculated from the ammonia excreted in the urine (and thus removed from the body) and the ammonia released into the renal vein (and thus added to the body ammonia pools). Subtraction of renal venous ammonia release from urinary ammonia excretion reveals that net renal ammonia addition to the body ammonia pools tended to be diminished in PCS-PF rats (122 and $34 \mathrm{nmole} \cdot 100 \mathrm{~g}$ body $\mathrm{wt}^{-1} \mathrm{~min}^{-1}$ at day 7 and 14 , respectively) compared with PF rats (260 and $180 \mathrm{nmole} \cdot 100 \mathrm{~g}$ body $\mathrm{wt}^{-1} \mathrm{~min}^{-1}$ at day 7 and 14 , respectively). In PCSBDL rats, the kidney turned into an organ of net ammonia disposal from the body $(\mathrm{P}<0.01$ versus $\mathrm{PF}$ and PCS-PF by ANOVA).

Glutamine (Figure 4; Table 1, 3-5) At day 7, arterial glutamine concentrations were significantly higher in PF than in NORM rats, and in PCSBDL compared with PF rats, but no differences were observed at day 14. Renal glutamine uptake was comparable in NORM and PF rats, and was comparably decreased in both hyperammonemic groups. Urinary glutamine excretion was enhanced in PCSBDL compared with both PF and PCS-PF rats. Kidney tissue glutamine concentrations were elevated in PCS-PF compared with PF, and in PCSBDL rats compared with PCS-PF rats.

Glutamate (Figure 5; Table 1, 3-5) Arterial glutamate levels were increased in PCS-PF compared with PF rats, and decreased in PCSBDL compared with PCS-PF rats. Glutamate release from the kidney was similar and did not differ from zero in most groups. Urinary glutamate excretion was enhanced in PCSBDL compared with both PCS-PF and PF rats. Kidney tissue glutamate was decreased in the PCSBDL group compared to both the PCS-PF 
Table 5. Urinary metabolite excretion and unine production.

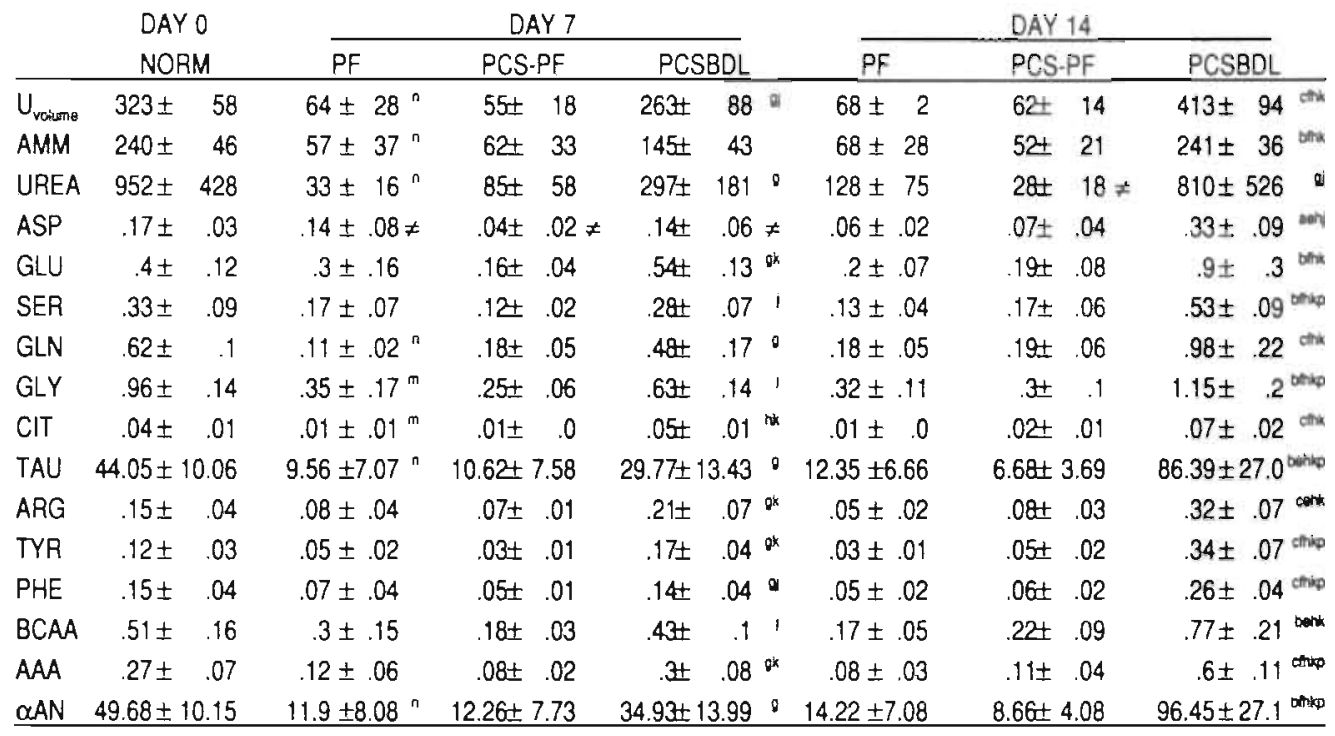

Data are means $\pm S E M$ in nmole $\cdot 100 \mathrm{~g}$ body $w^{-1} \cdot \mathrm{min}^{-1}$ per two kidneys, except urine production $\left(U_{\text {pouma }} \mu \mathrm{L} t^{-1}\right.$ per wo kidneys). For significance symbols, see Table 1. Complete list of amino acids available on request.

Table 6. Urinary metabolite excretion and arterial $\mathrm{pH}$ in conscious, unrestrained rats after 14 days of pair-feeding.

\begin{tabular}{|c|c|c|c|c|}
\hline & PF & PCS-PF & PCSBDL & \\
\hline $\mathrm{pH}_{\mathrm{ar}}$ & $7.402 \pm 0.006$ & $7.420 \pm 0.011$ & $7.433 \pm 0.011$ & $x$ \\
\hline $\mathrm{HCO}_{3}$ & $21.79 \pm 0.56$ & $21.04 \pm \quad 0.59$ & $21.76 \pm 1.06$ & \\
\hline$U_{v o l}$ & $33.62 \pm \quad 5.56$ & $33.51 \pm 6.75$ & $72.85 \pm 12.31$ & $x$ \\
\hline AMM & $273 \pm$ & $372 \pm$ & $749 \pm$ & If \\
\hline UREA & $1958 \pm$ & $1975 \pm$ & $2726 \pm 287$ & $y$ \\
\hline
\end{tabular}

Data are means \pm SEM in nmole $100 \mathrm{~g}$ body $w^{-1} \min ^{-1}$ per two kidneys, except unine production $\left(U_{\text {voume }} \mathrm{mLday}\right)$. Significance symbols: Mann Whitney $U, P<0.05$ : 'vs. PF, 'vs. PCS.

and PF group.

Remaining amino acids - important observations (Table 1, 3-5) Confirming previous reports, renal glycine uptake was observed $(11,38)$ and could account for approximately $50 \%$ of serine release (39) in most groups. It is interesting to note that in both chronic liver insufficiency groups, serine release was decreased. Renal citrulline uptake could account for the stoichiometric arginine release in all groups, confirming renal arginine biosynthesis previously observed in rats $(11,40)$ and humans $(39)$. In all groups a similar renal vein release of tyrosine was observed, concomitantly with phenylalanine uptake, which confirms previous suggestions derived from human studies that the kidney might play a role in the biosynthesis of tyrosine (39). An unexpected, novel finding was the observed renal BCAA 
release in all groups. Since the kidney has been shown to contain the enzymatic machinery to transform $\alpha$ ketoisocaproate to leucine (41), this could be the explanation.

Glucose and lactate (Table 1 \& 3) Arterial glucose concentrations were decreased in PCSBDL compared to the similar levels in all other groups. Lactate concentrations were elevated in PF compared with NORM rats and were comparably elevated in PCSBDL and PCS-PF rats compared with the $\mathrm{PF}$ group. Renal lactate uptake was similar in all groups.

Metabolic cage experiments (Table 6) In unrestrained, alert and freely moving rats, arterial $\mathrm{pH}$ was in the physiological range in all groups. No differences in $\mathrm{pH}$ were observed between groups, except for a slight increase in arterial $\mathrm{pH}$ in PCSBDL compared with PF rats. As observed already in anesthetized rats, urine production was similar in PF and PCS-PF rats, whereas it was significantly enhanced in the PCSBDL group. Also, urinary ammonia and urea excretion showed a similar pattern as observed during flux measurements under anesthesia.

\section{DISCUSSION}

In the present experiments we investigated the role of the kidney in ammonia and glutamine metabolism in two models of chronic liver insufficiency-induced hyperammonemia, because the kidney could theoretically play an important role in the adaptation to chronic hyperammonemia by enhancing ammonia disposal. Such an adaptation would require increased excretion of ammonia in the urine, either by enhanced renal uptake of blood-derived glutamine, or by diminished release of ammonia into the renal vein $(14,15)$.

Physiologically, renal ammonia production mainly results from the de-amidation of glutamine predominantly by intra-mitochondrial phosphate-dependent glutaminase $(12,14,42)$. Only minor quantities $( \pm 10 \%)$ are produced by the brush border membranebound $\gamma$-glutamyl transferase in the distal parts of the proximal tubule $(12,43)$. The values found in our study for renal vein ammonia release and urinary ammonia excretion in normal control rats are in agreement with data reported previously $(31,32)$.

In the present study, in both hyperammonemic groups at day 7 and 14 total apparent renal ammoniagenesis (flux + urinary excretion; Figure 3, upper panel) was diminished. Also, the fraction of total ammoniagenesis excreted in the urine (Figure 3, lower panel) was enhanced, i.e. the kidney adapted to hyperammonemia by changing the renal vein/ urine ammonia partition ratio. Thus, ammonia release into the renal vein was markedly decreased in both hyperammonemic groups at day 7 and 14 in comparison with the pair-fed sham operated 
Arterial

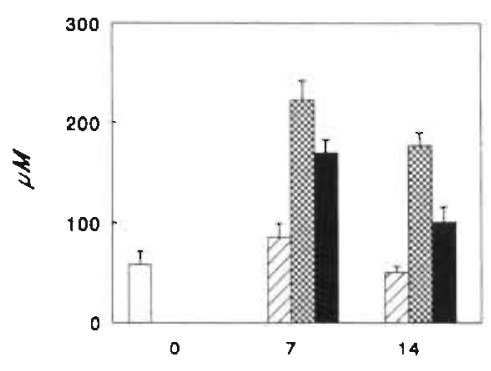

Urinary Excretion

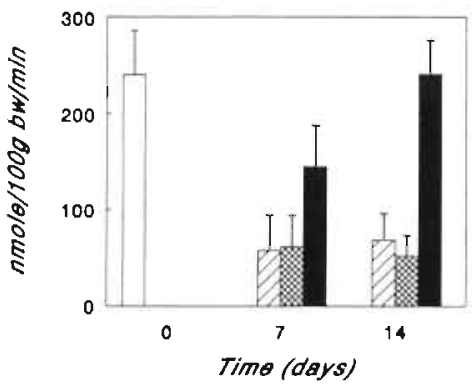

NORM

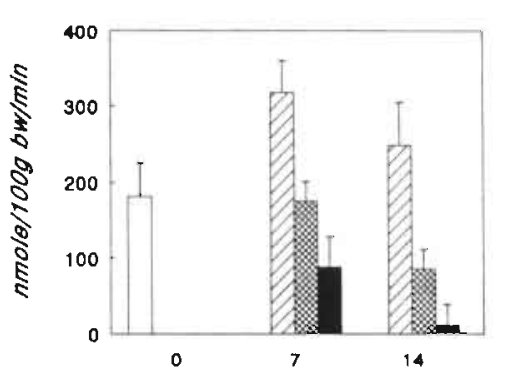

Kidney Concentration

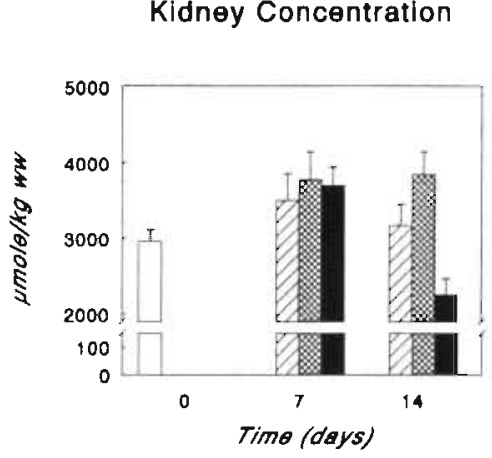

PCS-PF

Renal Flux
PCSBDL

Figure 2. Ammonia: arterial concentrations (left upper panel; $\mu \mathrm{M}$ ), renal flux (right upper panel; nmole $\cdot 100 \mathrm{~g}$ body $\mathrm{wt}^{-1} \cdot \mathrm{min}^{-1}$ per two kidneys), urinary excretion (left lower panel; nmole.100 g body $w^{-1} \mathrm{~min}^{-1}$ per two kidneys) and kidney tissue concentrations (right lower panel; $\mu$ mole $\mathrm{kg}$ wet $\mathrm{wt}^{-1}$ ) in normal control rats (NORM, open bars), sham operated pair-fed rats (PF, hatched bars), portacaval shunted rats (PCS-PF, cross hatched bars) and in rats with portacaval shunts and bile duct ligation (PCSBDL, filled bars). Data are means $\pm S E M ; N=6$ to 8 per group.

group. The diminution of renal vein ammonia release was more pronounced in the PCSBDL group. This suggests that the extent to which renal vein ammonia release is diminished, is related to the existence of hyperbilirubinemia or severity of liver insufficiency. Also, ammonia excretion in the urine was grossly increased in the PCSBDL group compared with the PCS-PF and PF group. These combined adaptations turned the kidney into an organ of net ammonia disposal from the body in the portacaval shunt/biliary obstruction group. Thus, in both chronic liver insufficiency models the kidney adapts to hyperammonemia either by reducing the net amount of ammonia added to the body pools, or by net removal of ammonia from the body.

Conflicting results have been reported concerning glutamine fluxes across the normal rat $(14,19-26,31,32)$ and human kidney (16). Most of these studies show net renal glutamine 
uptake, but some do not $(14,26)$. In this study, as well as in previous studies performed in our laboratory (Dejong et al., unpublished observation, 1992), the normal rat kidney proved to be an organ of net glutamine uptake. In both hyperammonemic groups at day 7 and day 14 , renal glutamine uptake was found to be reduced by more than $50 \%$, which was matched by the above-described reduction of total renal ammoniagenesis. This observation deserves some comment.

First, it was our initial hypothesis that chronic liver insufficiency-induced hyperammonemia would lead to hyperglutaminemia (by enhanced glutamine synthesis and release from glutamine synthetase-containing organs). Second, we hypothesized that this hyperglutaminemia would result in enhanced renal glutamine uptake and subsequent urinary ammonia excretion. However, although arterial glutamine levels were elevated in all operated groups compared to normal unoperated control rats, no differences in arterial glutamine levels were observed between the PCS-PF and PF groups, despite substantial differences in arterial ammonia levels. In fact, glutamine concentrations were only significantly higher in the PCSBDL group at day 7 . This seems to indicate that food intake and nutritional status are probably more important in regulating arterial glutamine levels than the existence of liver insufficiency or hyperammonemia. Also, since the elevated glutamine levels in the PCSBDL group at day 7 were not accompanied by an increased renal glutamine uptake, but instead led to diminished renal glutamine consumption, the conclusion seems justified that arterial glutamine levels are not an important determinant of renal glutamine uptake.

Although the urinary excretion of glutamine is strongly enhanced in the PCSBDL group compared with the PF and PCS-PF group especially at day 14, this does not constitute a major pathway of ammonia detoxification, because the amounts excreted are negligible compared with renal glutamine fluxes and arterial glutamine levels.

Renal tissue glutamine levels were elevated in PCS-PF rats compared with the PF and normal control group, and showed a further increase in the PCSBDL group. These changes in tissue glutamine occurred already at day 7 despite unchanged tissue ammonia concentrations. The reduced total renal ammoniagenesis in both hyperammonemic groups occurred despite elevated tissue glutamine levels (the main precursor for ammonia excretion). Also, tissue glutamine levels were elevated despite reduced renal glutamine uptake from the blood stream. These combined observations suggest that the glutaminase pathway is inhibited. This seems to be supported by the reduced glutamate and aspartate tissue concentrations in 


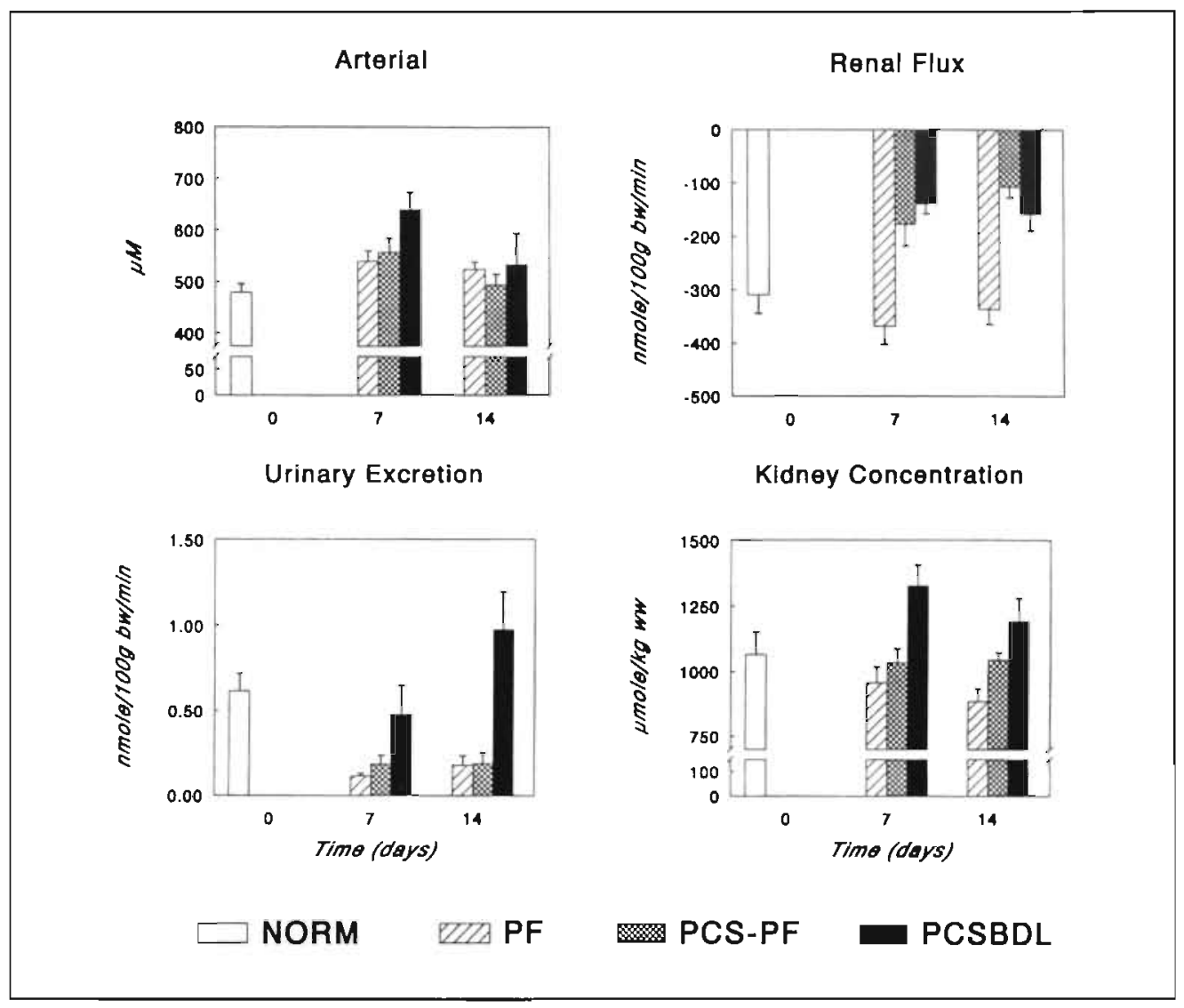

Figure 4. Glutamine: arterial concentrations (left upper panel; $\mu \mathrm{M}$ ), renal flux (right upper panel; nmole $100 \mathrm{~g}$ body wit' ${ }^{-1} \mathrm{~min}^{\cdot 1}$ per two kidneys), urinary excretion (left lower pane;; nmole.100 $\mathrm{g}$ body $\mathrm{wt}^{-1} \mathrm{~min}^{-1}$ per two kidneys) and kidney tissue concentrations (right lower panel; $\mu$ mole $\mathrm{kg}$ wet $\mathrm{wt}^{-1}$ ) in normal control rats (NORM, open bars), sham operated pair-led rats (PF, hatched bars), portacaval shunted rats (PCS-PF, cross hatched bars) and in rats with portacaval shunts and bile duct ligation ( $\mathrm{PCSBDL}$, filled bars). Data are means $\pm S E M ; \mathrm{N}=6$ to 8 per group.

both hyperammonemic groups, as well as the decreased tissue ammonia concentrations at day 14 in the PCSBDL group. Although glutaminase inhibition has been implicated previously in altered renal glutamine and ammonia metabolism (39), the mechanism of this postulated glutaminase inhibition is not clear: elevated ammonia $(11,44)$ and glutamate levels (44) are well-known inhibitors of glutaminase, but ammonia levels were similar in the $\mathrm{PF}$ and the hyperammonemic groups, and glutamate concentrations were diminished in the hyperammonemic groups. Also, since arterial $\mathrm{pH}$ and bicarbonate concentrations were similar in all groups (Table 6), this does not appear do be an important factor in regulating glutaminase activity. Further studies are necessary to elucidate the mechanisms underlying these observations. 
Arterial

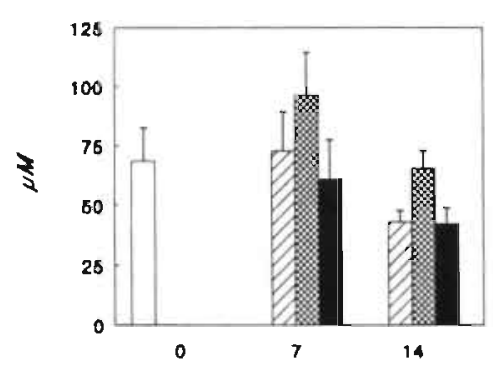

Urinary Excretion

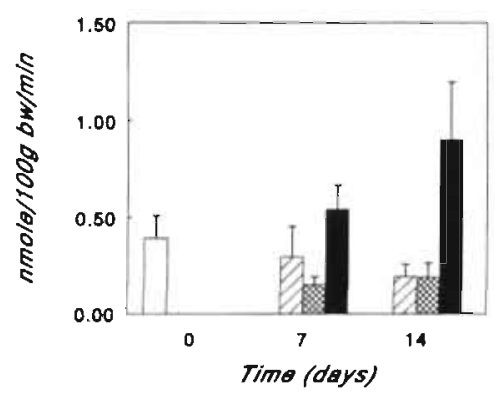

NORM
Renal Flux

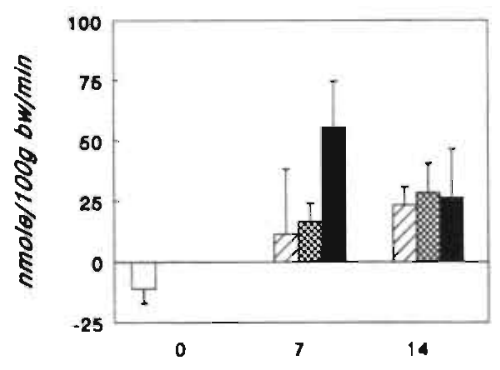

Kidney Concentration

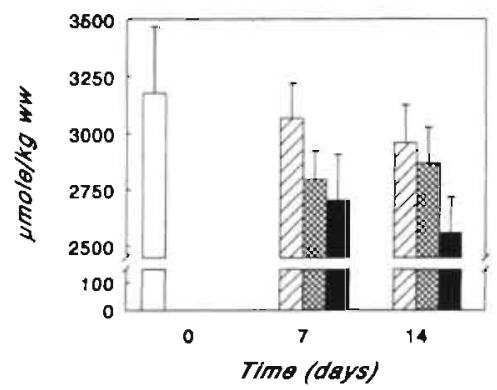

PCS-PF

PCSBDL

Figure 5. Glutamate: anerial concentrations (left upper panel; $\mu \mathrm{M}$ ), renal flux (right upper panel; nmole $\cdot 100 \mathrm{~g}$ body $\mathrm{wt}^{-1}-\mathrm{min}^{-1}$ per two kidneys), urinary excretion (left lower panel; nmole.100 $\mathrm{g}$ body $\mathrm{wt}^{-1}-\mathrm{min}^{-1}$ per two kidneys) and kidney tissue concentrations (right lower panel; $\mu$ mole $\mathrm{kg}$ wet $\mathrm{wt}^{-1}$ ) in normal control rats (NORM, open bars), sham operated pair-fed rats (PF, hatched bars), portacaval shunted rats (PCS-PF, cross hatched bars) and in rats with portacaval shunts and bile duct ligation (PCSBDL, filled bars). Data are means $\pm S E M ; N=6$ to 8 per group.

To exclude the possibility that some of the changes observed in urinary ammonia excretion were related to anesthesia or the PAH infusion, we extended the experiments to alert, freely moving rats. In addition, we attempted to relate in these rats the observed ammonia excretion to arterial acid-base parameters. The effects of acid-base changes on kidney ammonia and glutamine metabolism have been extensively studied in rats, dogs and humans (for review see e.g. (12,45-47)). Metabolic acidosis, whether acute or chronic, induces enhanced renal glutamine extraction $(16,19,22)$ and increased urinary ammonia excretion (16) at the expense of urea excretion (21), whereas total urinary nitrogen excretion as ammonia plus urea remains constant $(21,22)$. In contrast, respiratory alkalosis in cirrhotic patients was shown to enhance renal ammonia release into the circulation, thus reducing renal ammonia disposal (18). In the present experiments, we found in conscious unrestrained rats a similarly enhanced urinary ammonia excretion in the portacaval shunted/ 
biliary obstructed group as found in anesthetized rats, supporting the validity of our findings. The enhanced urinary ammonia excretion could be largely accounted for by increased urine production in both conscious, unrestrained and in anesthetized rats, probably related to the biliary obstruction (48). Also, the observed enhanced urinary ammonia excretion apparently was unrelated to acid-base changes, suggesting that the adaptive response of the kidney to chronic liver insufficiency-induced hyperammonemia occurs in a pH-independent way.

In conclusion, the present study shows that during chronic liver insufficiency-induced hyperammonemia the kidney plays an important role in the metabolic adaptation to hyperammonemia. This adaptive response is accomplished by reducing renal glutamine uptake and total renal ammonia production, and by increasing the fraction of total renal ammonia generation excreted in the urine at the expense of ammonia released into the renal vein, i.e. by increasing the urine-renal vein ammonia partition ratio. This adaptive response is probably not regulated by arterial $\mathrm{pH}$.

\section{REFERENCES}

1. Zieve L. Pathogenesis of hepatic encephalopathy. Metab Brain Dis 1987;2:147-165.

2. Meijer AJ, Lamers WH, Chamuleau RAFM. Nitrogen metabolism and omithine cycle function. Physiol Rev 1990;70:701-748.

3. Sherlock S. Diseases of the liver and biliary system. Blackwell Scientific Publications, Oxford, 8th Edition, 1989.

4. Mullen KD, McCullough AJ. Problems with animal models of chronic liver disease: Suggestions for improvement in standardization. Hepatology 1989:9:500-505.

5. Roughneen PT, Gouma DJ, Kulkarni AD, Fanslow WF, Rowlands BJ. Impaired specific cell-mediated immunity in experimental biliary obstruction and its reversibility by intemal biliary drainage. J Surg Res 1986;41:113-125.

6. Dunn CW, Horton JW, Megison SM, Vuitch MF. Contribution of portal systemic shunt to Kupffer cell dysfunction in obstructive jaundice. J Surg Res 1991;50:234-239.

7. Greve JW, Gouma DJ, Soeters PB, Buurman WA. Suppression of cellular immunity in obstructive jaundice is caused by endotoxins. A study in germ-free rats. Gastroenterology 1990;98:478-485.

8. Record CO. Neurochemistry of hepatic encephalopathy. Gut 1991;32:1261-1263.

9. Butterworth RF. Pathophysiology of hepatic encephalopathy; the ammonia hypothesis revisited. In: Bengtson F, Almdal T, Jeppsson B, Vilstrup H, eds. Progress in Hepatic Encephalopathy and Metabolic Nitrogen Exchange. CRC Press, Boca Raton, 1991:9-24.

10. Chance WT, Zhang FS, Foley Nelson T, Fischer JE. Hyperammonemia and anorexia in Morris hepatoma-bearing rats. Physiol Behav 1991;50:397-401.

11. Newsholme EA, Leech AR. Biochemistry for the Medical sciences. John Wiley \& Sons, New York, 1983.

12. Tannen RL. Ammonia metabolism. Am J Physiol 1978;235:F265-277.

13. Tyor MP, Owen EE, Berry JN, Flanagan JF. The relative role of extremity, liver and kidney as ammonia receivers and donors in patients with liver disease. Gastroenterology 1960;39:420-424.

14. Yablon SB, Relman AS. Glutamine as a regulator of renal ammonia production in the rat [abstract]. Kidney Int 1977; 12:546.

15. Owen EE, Johnson JH, Tyor MP. The effect of induced hyperammonemia on renal ammonia metabolism. J Clin lnvest 1961;40:215-221.

16. Owen EE, Robinson RR. Amino acid extraction and ammonia metabolism by the human kidney during the prolonged administration of ammonium chloride. J Clin lnvest 1963;42:263-276.

17. Welbourne $T$, Weber $M$, Bank $N$. The effect of glutamine administration on urinary ammonium excretion in normal subjects and patients with renal disease. J Clin Invest 1972;51:1852-1860.

18. Berry JN, Flanagan JF, Owen EE, Tyor MP. The kidney as a source of blood ammonia in resting and hyperventilated cirrhotics. Clin Res 1959;7:154-155.

19. Phromphetcharat V, Welboume TC. Renal glutamine extraction and gut/liver interaction in glutamine homeostasis. Contrib Nephrol 1985;47:9-14. 
20. Welboume TC. Effect of metabolic acidosis on hindquarter glutamine and alanine release. Metabolism 1986;35:614-618.

21. Welboume TC, Phromphetcharat V, Givens G, Joshi S. Regulation of interorgan glutamine flow in metabolic acidosis. Am J Physiol 1986;250:E457-E463.

22. Welbourne TC, Childress D, Givens $G$. Renal regulation of interorgan glutamine flow in metabolic acidosis. Am J Physiol 1986;251:R858-R866.

23. Welboume TC. Hepatic glutaminase flux regulation of glutamine homeostasis. Studies in vivo. Biol Chem Hoppe Seyler 1986;367:301-305.

24. Welboume TC, Dass PD. Gamma glutamyltransferase contribution to renal ammoniagenesis in vivo. Pflügers Arch 1988;411:573-578.

25. Vinay P, Allignet E, Pichette C, Watford M, Lemieux G, Gougoux A. Changes in renal metabolite profile and ammoniagenesis during acute and chronic metabolic acidosis in dog and rat. Kidney Int 1980;17:312-325.

26. Schröck H, Goldstein L. Interorgan relationships for glutamine metabolism in normal and acidotic rats. Am J Physiol 1981;240:E519-E525.

27. Ribeiro J, Nordlinger B, Ballet F, Cynober L, Coudray-Lucas C, Baudrimont M, Legendre C, Delelo $\mathrm{R}$, Panis $\mathrm{Y}$. Intrasplenic hepatocellular transplantation corrects hepatic encephalopathy in portacaval shunted rats. Hepatology 1992;15:12-18.

28. Maddison JE, Dodd PR, Morrison M, Johnston GAR, Farrell GC. Plasma GABA, GABA-like activity and the brain GABA-benzodiazepine receptor complex in rats with chronic hepatic encephalopathy. Hepatology 1987:7:621-628.

29. Dejong CHC, Kampman MT, Deutz NEP, Soeters PB. Altered glutamine metabolism in rat portal drained viscera and hindquarter during hyperammonemia. Gastroenterology 1992;102:936-948.

30. Farrow NA, Kanamori K, Ross BD, Parivar F. A ${ }^{15} \mathrm{~N}-\mathrm{NMR}$ study of cerebral, hepatic and renal nitrogen metabolism in hyperammonemic rats. Bioch J 1990;270:473-481.

31. Warter JM, Imler M, Marescaux C, Chabrier G, Rumbach L, Micheletti G, Krieger J. Sodium valproate-induced hyperammonemia in the rat: role of the kidney. Eur J Pharmacol 1983;87:177-182.

32. Imler $M$, Chabrier $G$, Marescaux $C$, Warter JM. Effects of 2,4-dinitrophenol on renal ammoniagenesis in the rat. Eur J Pharmacol 1986;123:175-179.

33. van Eijk $\mathrm{HMH}$, van der Heijden $\mathrm{MAH}$, van Berlo CLH, Soeters PB. Fully automated liquid-chromatographic determination of amino acids. Clin Chem 1988;34:2510-2513.

34. Dejong CHC, Kampman MT, Deutz NEP, Soeters PB. Cerebral cortex ammonia and glutamine metabolism during liver insufficiency-induced hyperammonemia in the rat. $\mathrm{J}$ Neurochem 1992;59:1071-1079.

35. Selkurt EE. Measurement of renal blood flow. Meth Med Res 1948;1:191-199.

36. Dejong CHC, Deutz NEP. Soeters PB. Inter-organ nitrogen exchange during prolonged starvation in the rat. J Clin Nutr Gastroenterol 1991;6:176-183.

37. Norusis MJ. SPSS/PC+V3.1 BASE MANUAL for the IBM PC/XT/AT and PS/2. SPSS Inc, Chicago, USA, 1989.

38. SilbernagI S. The renal handling of amino acids and oligopeptides. Physiol Rev 1988;68:911-1007.

39. Tizianello A, de Ferrari G, Garibotto G, Gurreri G, Robaudo C. Renal metabolism of amino acids and ammonia in subjects with normal renal function and in patients with chronic renal insufficiency. $\mathrm{J} C l$ in Invest 1980;65:1162-1173.

40. Cooper AJL, Filc-DeRicco S, Gelbard AS. L- ${ }^{13} \mathrm{~N}$ glutamate metabolism in normal rat kidney. In: Bengtson F, Almdal T, Jeppsson B, Vilstrup H, eds. Progress in Hepatic Encephalopathy and Metabolic Nitrogen Exchange. CRC Press, Boca Raton, 1991:341-351.

41. Abumrad NN, Wise KL. Williams PE, Abumrad NA, Lacy WW. Disposal of alpha-ketoisocaproate: roles of liver, gut, and kidneys. Am J Physiol 1982;243:E123-E131.

42. Good DW. Knepper MA. Mechanisms of ammonium excretion: Role of the renal medulla. Semin Nephrol 1990;10:166-173.

43. Simon EE, Merli C. Hemdon J, Hamm LL. Contribution of luminal ammoniagenesis to proximal tubule ammonia appearance in the rat. Am J Physiol 1990;259;F402-F407.

44. Nelson D, Rumsey WL, Erecinska M. Glutamine catabolism by heart muscle. Biochem J 1992:282:559-564.

45. Halperin ML, Ethier JH, Kamel KS. The excretion of ammonium ions and acid base balance. Clin Biochem 1990;23:185-188.

46. Schoolwerth AC. Regulation of renal ammoniagenesis in metabolic acidosis. Kidney Int 1991:40:961-973. 
47. Chobanian MC. Hormonal control of renal ammonium metabolism: a review. Am J Kidney Dis 1989;14:248-252.

48. O'Neill PA, Wait RB, Kahng KU. Role of sympathetic nerve activity in renal failure associated with obstructive jaundice in the rat. Am J Surg 1991;161:662-667. 

Chapter 12

\section{Summary and conclusions}

Liver failure constitutes an important cause of morbidity and mortality, and adversely affects the outcome of any surgical procedure. Liver failure is commonly associated with impairment of several detoxification processes, inducing among others profound disturbances in nitrogen homeostasis. A crucial feature in this context is the diminished hepatic urea synthesis capacity, leading to an impaired capacity to detoxify ammonia, which in turn contributes to the development of systemic hyperammonemia. Because glutamine synthesis has been suggested to be the most important alternative pathway for ammonia detoxification during diminished urea synthesis, systemic hyperammonemia in turn has a considerable impact on the metabolism of the non-essential amino acid glutamine. Although the relation between ammonia and glutamine metabolism in several organs has received considerable attention, virtually no attention has been paid to the interaction between these organs during liver failure. Especially, no attempts have been made to quantify the exchange of nitrogen and specifically ammonia and glutamine between organs. Therefore, the principal aim of this thesis was to study the mass flux (=net uptake or output) of ammonia and glutamine between several organs.

This thesis comprises three major sections. The first section (chapters 1-4) represents an introduction to the measurements of interorgan mass fluxes of glutamine and ammonia during acute and chronic liver failure. The second part (chapters 5-7) describes inter-organ ammonia and glutamine mass flux measurements during acute liver failure. The final section (chapter 8-11) describes the inter-organ mass flux measurements during chronic liver failure.

In chapter 1 a summary of the relevant literature concerning ammonia and glutamine metabolism in humans and experimental animals is given. The first part of this chapter gives some biochemical aspects of ammonia and glutamine, and the key reactions in their synthesis and breakdown, as well as a detailed description of the current opinion on ammonia and glutamine metabolism in various organs in the physiological situation. The second part of this chapter summarizes the general opinion on ammonia and glutamine metabolism in various organs during liver disease.

In chapter 2 several experimental animal models of acute and chronic liver failure are briefly reviewed. The (dis-) advantages and problems of several of these models are discussed. The 
motivation for the choice of the animal models used in this thesis is given and the animal models are defined in detail. Furthermore, some technical aspects and theoretical backgrounds of blood flow and flux measurement across several organs are addressed.

Chapter 3 describes the inter-organ flux between muscle, gut and liver of glutamine and ammonia, as well as several other metabolites during a 4-day starvation period in the rat. It is shown that skeletal muscle glutamine release increased to a maximum on the third day of starvation, whereas intestinal glutamine uptake decreased at day 3 . During starvation, the liver switched from net glutamine release to glutamine uptake at day 2, coinciding with maximal hepatic urea release. Because arterial glutamine levels did not change, although muscle glutamine release decreased at day 4 and intestinal glutamine uptake decreased at day 3 and 4 , it must be concluded from these experiment that other organs are important in the regulation of arterial glutamine concentrations.

Experiments to investigate the interaction between the major glutamine producing organ (muscle) and the major glutamine consuming organs (gut and liver) are presented in chapter 4. Fluxes of ammonia, glutamine and several other metabolites were measured across gut, muscle and liver. To investigate whether gut glutamine consumption is an important regulator of muscle glutamine production, organ fluxes were measured in rats 40 hours after $80 \%$ enterectomy, thereby eliminating a major glutamine consuming organ, as well as in controls. Because muscle glutamine production did not change despite reduced intestinal and splanchnic glutamine uptake, it must be concluded that splanchnic glutamine uptake is not a major regulator of muscle glutamine production.

Chapter 5 describes the interaction between skeletal muscle and intestinal glutamine and ammonia metabolism during acute hyperammonemia, induced by acute liver ischemia as well as in portacaval shunted controls. These experiments show that no significant net ammonia uptake by muscle occurred in this model of severe acute hyperammonemia nor in portacaval shunted rats. Muscle glutamine release decreased in acute liver ischemia rats compared with portacaval shunted rats, although intracellular ammonia and glutamine concentrations were enormously increased. This suggests an inhibition of glutamine release from muscle. Simultaneously, intestinal ammonia release increased during acute liver ischemia compared with portacaval shunted controls, whereas glutamine uptake decreased during liver ischemia and finally switched to glutamine release.

Chapter 6 consists of two parts. In part A, a new method for repeated in vivo cerebral cortex flux measurements and its validation is described. In part B, this new method is applied to the in vivo measurement of glutamine and ammonia across the cerebral cortex in rats with acute liver ischemia as well as in portacaval shunted controls. It is shown that the cerebral cortex of the rat took up ammonia during acute severe hyperammonemia induced by liver ischemia. This ammonia uptake was accompanied by glutamine release from the cerebral cortex during the later phases of liver ischemia. This suggests that the cerebral cortex of the rat contributes to ammonia detoxification by synthesizing glutamine during severe acute hyperammonemia. The consequently increased glutamate consumption might be related to the observed decrease in tissue glutamate concentrations, which might adversely affect excitatory neurotransmission. The simultaneously observed increased tissue GABA levels 
during acute liver ischemia could point to enhanced inhibitory GABAergic neurotransmission, or alternatively to increased flux through the GABA-shunt pathway.

The role of the kidney in ammonia and glutamine metabolism during acute liver failure and the related hyperammonemia is investigated in chapter 7 by measuring renal fluxes, urinary excretion and renal tissue concentrations in rats with acute liver ischemia and in portacaval shunted and normal controls. It is shown that in portacaval shunted rats the kidney played an important role in ammonia disposal from the body by diminishing glutamine uptake and total renal ammoniagenesis and by enhancing the fraction of total ammoniagenesis excreted in the urine at the expense of ammonia released into the renal vein. These changes in ammonia handling turned the kidney from an organ of ammonia production in the body in the normal rat, to an organ of ammonia disposal in the portacaval shunted rats. However, because renal ammonia disposal was similar in portacaval shunted rats and acute liver ischemia rats, despite much more severe hyperammonemia in the latter group, it is concluded that renal ammonia disposal capacity is limited.

Chapter 8 describes the role of skeletal muscle in ammonia and glutamine metabolism in two models of chronic liver failure as well as in controls. Portacaval shunting and portacaval shunting combined with bile duct ligation were used as models of chronic liver failure. To reduce the effects of differences in food intake, all rats received similar amounts of food. It is shown that in both hyperammonemic chronic liver insufficiency groups, no net ammonia uptake by skeletal muscle occurred. Yet, skeletal muscle ammonia levels were largely increased during hyperammonemia. Despite these increased skeletal muscle ammonia levels, muscle glutamine concentrations were similar in hyperammonemic and in pair-fed control rats. Yet, glutamine release was largely increased in the hyperammonemia groups. These results suggest that during stable hyperammonemia there is no strict stoichiometry between muscle ammonia uptake and glutamine release. The results of this chapter suggest that nutritional factors are crucial in the correct interpretation of results obtained in chronic liver failure models.

The role of intestinal glutamine and ammonia metabolism during chronic liver failure was studied in the described models of chronic liver insufficiency. The results are described in chapter 9 and suggest that under conditions of well-controlled food intake, intestinal ammonia generation in rats with chronic liver failure remains unchanged despite alterations in intestinal glutamine uptake. This suggests that arterial ammonia levels in these models are probably mainly set by the existence of a portacaval shunt rather than by increased gut glutamine consumption and ammonia liberation.

Chapter 10 describes the results of studies on the role of the cerebral cortex in ammonia and glutamine metabolism in the above-described two models of chronic liver failure in the rat. The flux measurement method described in chapter $6 \mathrm{~A}$ was used. It is demonstrated that during chronic, stable, liver insufficiency-induced hyperammonemia, the cerebral cortex continuously took up small amounts of ammonia, contributing to the elevated tissue ammonia levels. Simultaneously, cerebral cortex glutamine concentrations were increased in both chronic liver failure models, accompanied by a continuous release of small amounts 
of glutamine. Although it has repeatedly been suggested in literature that this process of ammonia uptake, glutamine synthesis and glutamine release from the brain could cause a decrease in cerebral cortex tissue glutamate concentrations, contributing to a neurotransmitter imbalance, we found similar cerebral cortex tissue glutamate levels in rats with chronic liver failure and hyperammonemia and in pair-fed control rats with no hyperammonemia. From this it is concluded that nutritional factors or standard surgical trauma might contribute to these decreased cerebral cortex tissue glutamate levels.

The role of the kidney in ammonia and glutamine metabolism during chronic liver failure is described in chapter 11. In these studies in the described two models of chronic liver failure, it is shown that the kidney plays a very important role in the adaptation to chronic liver insufficiency-induced hyperammonemia by reducing renal glutamine uptake and total renal ammoniagenesis, and by enhancing the fraction of total ammoniagenesis excreted in the urine.

\section{GENERAL CONCLUSION}

It is generally believed that glutamine synthesis, from equal amounts of ammonia and glutamate, is the most important alternative pathway of ammonia detoxification during acute and chronic liver failure. Skeletal muscle has long been thought to be the most important organ in this alternative detoxification pathway, by taking up ammonia and releasing glutamine. The results presented in chapters 5 and 8 suggest that the role of skeletal muscle as an ammonia detoxification organ is generally overestimated.

As to the role of the brain, it is confirmed that the brain takes up ammonia and releases glutamine during acute (chapter 6) and chronic liver failure (chapter 10) and the related hyperammonemia. Although this constitutes a means of ammonia detoxification, the amounts of glutamine release are very small. Therefore this mechanism cannot be considered important in maintaining nitrogen homeostasis during liver failure. On the contrary, the consequently increased glutamate consumption in the detoxification of ammonia in brain might adversely affect excitatory neurotransmission.

The intestines show enhanced ammonia release during severe acute liver insufficiencyinduced hyperammonemia, whereas glutamine uptake diminishes and finally reverses to glutamine release (chapter 5). The latter phenomenon might be related to inhibition of glutaminase by high ammonia concentrations, leading to an inhibition of glutamine consumption and resulting in intestinal mucosa cell dysfunction. During chronic liver failure (chapter 9), intestinal ammonia release is not enhanced, suggesting that in this situation, the gut does not aggravate hyperammonemia by enhancing glutamine uptake and ammonia release.

The results of chapter $7 \& 11$ show that the role of the kidney during acute and chronic hyperammonemia is probably an underestimated one. Both during acute and chronic hyperammonemia, the kidney reduces total ammoniagenesis and enhances the fraction of total renal ammoniagenesis excreted in the urine. Thereby the kidney reverses from an organ of ammonia production in the normal rat to an organ of ammonia disposal in the hyperammonemic rat. The results of chapter 7 suggest that during severe acute liver ischemia-induced hyperammonemia the capacity of the kidney to excrete ammonia is exceeded. 
The observation during acute severe liver failure that muscle glutamine release is not increased, but instead decreased, combined with the simultaneously observed huge increase in arterial glutamine levels, raises the question of the source of the arterial glutamine. The minimal amounts of glutamine released from the brain in the same model cannot explain the arterial glutamine levels either. Diminished intestinal glutamine uptake also is not a sufficient explanation, because arterial glutamine levels are already elevated when gut glutamine consumption is still unchanged. Since renal glutamine uptake did not change during acute liver insufficiency-induced hyperammonemia, maybe enhanced glutamine production in the lungs or the heart, or diminished consumption in the immune system could play a role in these elevated arterial glutamine levels.

Finally, it appears from the results obtained in this thesis that glutaminase inhibition might be more important than glutamine synthetase stimulation in explaining several metabolic alterations during acute and chronic liver failure. 
s 


\section{Chapter 12}

\section{Samenvatting en conclusies}

Leverfalen vormt een belangrijke oorzaak van morbiditeit en mortaliteit, en heeft een negatief effect op het eindresultaat van nagenoeg elke chirurgische procedure. Leverfalen is doorgaans geassocieerd met een belemmering van verscheidene ontgiftingsprocessen, hetgeen onder meer resulteert in een diepgaande verstoring van de stikstof-homeostase. Cruciaal in deze context is de verminderde ureumsynthese-capaciteit met daaruit voortvloeiende verminderde ammoniak-ontgiftingscapaciteit, welke bijdraagt tot het ontstaan van systemische hyperammoniëmie. Aangezien de synthese van het niet essentiële aminozuur glutamine wel gezien wordt als de belangrijkste alternatieve ammoniak-ontgiftingsroute gedurende verminderde ureumsynthese-capaciteit, is het aannemelijk dat systemische hyperammoniëmie een grote invloed heeft op het glutamine metabolisme. Hoewel de relatie tussen ammoniak- en glutaminemetabolisme in verscheidene organen tijdens leverfalen veel aandacht heeft gekregen in het verleden, is er nagenoeg geen onderzoek verricht naar de interacties tussen organen gedurende leverfalen. In het bijzonder zijn er geen pogingen ondernomen om de uitwisseling van stikstof en speciaal ammoniak en glutamine tussen organen te kwantificeren gedurende leverfalen. Het hoofddoel van dit proefschrift was dan ook het bestuderen van de flux (=netto opname of afgifte) van ammoniak en glutamine tussen verscheidene organen tijdens leverfalen.

Dit proefschrift omvat in essentie drie delen. In het eerste deel (Hoofdstuk 1-4) wordt een introductie gegeven op de meting van interorgaan-flux van glutamine en ammoniak gedurende acuut en chronisch leverfalen. In het tweede deel (Hoofdstuk 5-7) wordt de meting van interorgaan-flux gedurende acuut leverfalen beschreven. Het laatste deel (Hoofdstuk 8-11) beschrijft de metingen van de interorgaan-flux gedurende chronisch leverfalen.

In hoofdstuk 1 wordt een review gegeven van de relevante literatuur op het gebied van ammoniak- en glutaminemetabolisme in humaan en dierexperimenteel onderzoek. Het eerste deel van het hoofdstuk beschrijft enkele biochemische aspecten van ammoniak en glutamine, en de sleutelreacties in hun afbraak en synthese. Tevens wordt een gedetailleerde beschrijving gegeven van de vigerende communis opinio aangaande ammoniak- en glutaminemetabolisme in diverse organen in de fysiologische situatie. Het tweede deel van dit hoofdstuk geeft een overzicht van de huidige theorieën met betrekking tot ammoniak- en glutaminemetabolisme gedurende leverfalen.

In hoofdstuk 2 wordt een review gegeven van de verscheidene in gebruik zijnde diermodellen voor acuut en chronisch leverfalen. De voor- en nadelen van deze modellen worden 
van in vivo flux van ammoniak en glutamine over de cortex cerebri in ratten met acute leverischemie en in portocavaal geshunte ratten. Deze experimenten tonen dat de cortex cerebri van de rat ammoniak opneemt tijdens acute emstige hyperammoniëmie, geïnduceerd door acute lever-ischemie. Deze ammoniak-opname wordt in de latere stadia van acute leverischemie gevolgd door een afgifte van glutamine door de cortex cerebri. Dit suggereert dat de cortex cerebri van de rat bijdraagt aan de ontgifting van ammoniak door het synthetiseren van glutamine tijdens acute lever-ischemie. Een daaruit voortvloeiende consumptie van de excitatoire neurotransmitter glutamaat zou de waargenomen gedaalde weefselconcentraties van glutamaat tijdens acute lever-ischemie kunnen verklaren en zou kunnen leiden tot een beinvloeding van de excitatoire neurotransmissie. De tegelijkertijd waargenomen stijging van de weefselconcentraties van de inhibitoire neurotransmitter GABA zou enerzijds kunnen wijzen op een toegenomen GABA-erge neurotransmissie, of anderzijds op een toegenomen flux door de GABA-shunt.

De rol van de nier in het ammoniak- en glutamine metabolisme gedurende acuut leverfalen wordt beschreven in hoofdstuk 7. De flux van ammoniak en glutamine over de nier, de weefsel concentraties van ammoniak en glutamine in de nier en de uitscheiding van deze metabolieten in de urine werd gemeten tijdens acute lever-ischemie, portocavale shunting en in controle ratten. Tijdens portocavale shunting bleek de nier een belangrijke rol te spelen in de aanpassing aan hyperammoniëmie. De glutamine opname door de nier en de totale ammoniak-produktie (afgifte naar de niervene + uitscheiding in de urine) verminderde en van de totale ammoniak-produktie bleek tijdens door portocavale shunting geïnduceerde hyperammoniëmie een grotere fractie in de urine uitgescheiden te worden, en de hoeveelheid ammoniak, die naar de niervene werd afgegeven, verminderde. Door deze veranderingen in ammoniak-metabolisme bleek de nier tijdens hyperammoniëmie te veranderen van een orgaan, dat in normale ratten netto ammoniak aan de lichaamspools toevoegde, in een orgaan, dat tijdens hyperammoniëmie netto ammoniak aan de lichaamspools onttrok. Echter, de totale hoeveelheid ammoniak, die netto door de nier aan de lichaamspools werd onttrokken, was gelijk in ratten met acute lever-ischemie en ratten met een portocavale shunt. Hieruit kan geconcludeerd worden dat de ammoniak-excretiecapaciteit van de nier beperkt is.

Hoofdstuk 8 beschrijft de rol van de skeletspier in ammoniak- en glutamine-metabolisme gedurende chronisch leverfalen en in controle ratten. Portocavale shunting en portocavale shunting gecombineerd met galwegobstructie werden gebruikt als model voor chronisch leverfalen. Om de effecten van verschillen in voedselinname zoveel mogelijk te reduceren werd aan alle ratten een gelijke hoeveelheid voer verstrekt. In beide modellen van hyperammoniëmie en leverfalen bleek de skeletspier geen significante netto opname van ammoniak te vertonen. Toch waren de weefselconcentraties van ammoniak in de skeletspier fors gestegen tijdens chronisch leverfalen. Ondanks deze gestegen ammoniak-concentraties in de spier waren de glutamine-concentraties vergelijkbaar in de skeletspieren van hyperammoniemische en paarsgewijs gevoede controle ratten, hetgeen suggereert dat er gedurende stabiele langdurige hyperammoniëmie geen stoichiometrie is tussen ammoniak-opname en glutamine-afgifte door de skeletspier. Tevens lijken de resultaten in dit hoofdstuk erop te 
van in vivo flux van ammoniak en glutamine over de cortex cerebri in ratten met acute leverischemie en in portocavaal geshunte ratten. Deze experimenten tonen dat de cortex cerebri van de rat ammoniak opneemt tijdens acute emstige hyperammoniëmie, geïnduceerd door acute lever-ischemie. Deze ammoniak-opname wordt in de latere stadia van acute leverischemie gevolgd door een afgifte van glutamine door de cortex cerebri. Dit suggereert dat de cortex cerebri van de rat bijdraagt aan de ontgifting van ammoniak door het synthetiseren van glutamine tijdens acute lever-ischemie. Een daaruit voortvloeiende consumptie van de excitatoire neurotransmitter glutamaat zou de waargenomen gedaalde weefselconcentraties van glutamaat tijdens acute lever-ischemie kunnen verklaren en zou kunnen leiden tot een beinvloeding van de excitatoire neurotransmissie. De tegelijkertijd waargenomen stijging van de weefselconcentraties van de inhibitoire neurotransmitter GABA zou enerzijds kunnen wijzen op een toegenomen GABA-erge neurotransmissie, of anderzijds op een toegenomen flux door de GABA-shunt.

De rol van de nier in het ammoniak- en glutamine metabolisme gedurende acuut leverfalen wordt beschreven in hoofdstuk 7. De flux van ammoniak en glutamine over de nier, de weefsel concentraties van ammoniak en glutamine in de nier en de uitscheiding van deze metabolieten in de urine werd gemeten tijdens acute lever-ischemie, portocavale shunting en in controle ratten. Tijdens portocavale shunting bleek de nier een belangrijke rol te spelen in de aanpassing aan hyperammoniëmie. De glutamine opname door de nier en de totale ammoniak-produktie (afgifte naar de niervene + uitscheiding in de urine) verminderde en van de totale ammoniak-produktie bleek tijdens door portocavale shunting geïnduceerde hyperammoniëmie een grotere fractie in de urine uitgescheiden te worden, en de hoeveelheid ammoniak, die naar de niervene werd afgegeven, verminderde. Door deze veranderingen in ammoniak-metabolisme bleek de nier tijdens hyperammoniëmie te veranderen van een orgaan, dat in normale ratten netto ammoniak aan de lichaamspools toevoegde, in een orgaan, dat tijdens hyperammoniëmie netto ammoniak aan de lichaamspools onttrok. Echter, de totale hoeveelheid ammoniak, die netto door de nier aan de lichaamspools werd onttrokken, was gelijk in ratten met acute lever-ischemie en ratten met een portocavale shunt. Hieruit kan geconcludeerd worden dat de ammoniak-excretiecapaciteit van de nier beperkt is.

Hoofdstuk 8 beschrijft de rol van de skeletspier in ammoniak- en glutamine-metabolisme gedurende chronisch leverfalen en in controle ratten. Portocavale shunting en portocavale shunting gecombineerd met galwegobstructie werden gebruikt als model voor chronisch leverfalen. Om de effecten van verschillen in voedselinname zoveel mogelijk te reduceren werd aan alle ratten een gelijke hoeveelheid voer verstrekt. In beide modellen van hyperammoniëmie en leverfalen bleek de skeletspier geen significante netto opname van ammoniak te vertonen. Toch waren de weefselconcentraties van ammoniak in de skeletspier fors gestegen tijdens chronisch leverfalen. Ondanks deze gestegen ammoniak-concentraties in de spier waren de glutamine-concentraties vergelijkbaar in de skeletspieren van hyperammoniemische en paarsgewijs gevoede controle ratten, hetgeen suggereert dat er gedurende stabiele langdurige hyperammoniëmie geen stoichiometrie is tussen ammoniak-opname en glutamine-afgifte door de skeletspier. Tevens lijken de resultaten in dit hoofdstuk erop te 
wijzen dat voedingsfactoren cruciaal zijn in de interpretatie van resultaten verkregen in modellen van chronisch leverfalen.

De rol van de darm in ammoniak- en glutamine-metabolisme tijdens chronische hyperammoniëmie in de bovenbeschreven modellen van chronisch leverfalen wordt beschreven in hoofdstuk 9 . Uit de resultaten in dit hoofdstuk blijkt duidelijk dat onder omstandigheden van goed gecontroleerde voedselinname de afgifte van ammoniak door de darm niet verandert gedurende chronisch leverfalen, ondanks veranderingen in intestinale glutamine opname. Dit suggereert dat de arteriële concentraties van ammoniak in deze modellen van chronisch leverfalen meer bepaald worden door het bestaan van een portocavale shunt, dan door toegenomen glutamine-opname of ammoniak-produktie door de darm.

Hoofdstuk 10 beschrijft de resultaten van experimenten, waarin de rol van de cortex cerebri van de rat in ammoniak- en glutamine-metabolisme gedurende chronisch leverfalen in de bovengenoemde modellen werd onderzocht. De methode van flux meting over de cortex cerebri, zoals beschreven in hoofdstuk 6A, werd hiervoor gebruikt. Uit de resultaten blijkt, dat gedurende stabiele chronische hyperammoniëmie de cortex cerebri van de rat continu een kleine hoeveelheid ammoniak opnam, waardoor de weefselconcentraties van ammoniak stegen. Tegelijkertijd stegen de weefselconcentraties van glutamine in beide modellen van chronisch leverfalen, leidend tot een continue afgifte van een kleine hoeveelheid glutamine door de cortex cerebri naar de bloedbaan. Hoewel bij herhaling in de literatuur gesuggereerd is dat dit proces van ammoniak-opname, glutamine-synthese en glutamineafgifte door de cortex cerebri een daling van de glutamaat-concentraties in de cortex cerebri tot gevolg zou kunnen hebben, hetgeen zou kunnen bijdragen tot een vermindering van de excitatoire neurotransmissie, vonden wij gelijke glutamaat-concentraties in de cortex cerebri van ratten met chronisch leverfalen en paarsgewijs gevoede controle ratten. Hieruit kan geconcludeerd worden, dat voedingsfactoren en standaard operatie-trauma zouden kunnen bijdragen tot het ontstaan van deze gedaalde glutamaatconcentraties.

De rol van de nier in ammoniak en glutamine-metabolisme gedurende chronisch hyperammoniëmie, door leverfalen veroorzaakt, wordt beschreven in hoofdstuk 11. In de experimenten tijdens chronisch leverfalen blijkt dat de nier een belangrijke rol speelt in de aanpassing aan chronische hyperammoniëmie door een vermindering van de glutamine opname en totale ammoniagenese door de nier en een toename van de fractie van de totale ammoniak produktie die wordt uitgescheiden in de urine.

\section{SLOTCONCLUSIE}

Algemeen wordt aangenomen, dat glutamine-synthese uit gelijke hoeveelheden glutamaat en ammoniak de belangrijkste alternatieve ammoniakontgiftingsroute is gedurende acuut en chronisch leverfalen. Lang is gedacht, dat de skeletspier het belangrijkste orgaan is, waar deze alternatieve ontgifting van ammoniak plaatsvindt door opname van ammoniak en afgifte van glutamine. De resultaten van hoofdstuk 5 en 8 suggereren dat de rol van de skeletspier in ammoniak-ontgifting tijdens leverfalen doorgaans wordt overschat. Er blijkt geen netto ammoniak-opname plaats te vinden. 
Wat betreft de rol van de hersenen vormt dit proefschrift een bevestiging van de vigerende theorie dat de hersenen bijdragen aan ammoniak-ontgifting door ammoniakopname en glutamine-release zowel tijdens acute (Hoofdstuk 6) als tijdens chronische (Hoofdstuk 10) hyperammoniëmie. Hoewel dit gezien kan worden als een manier van ammoniak-ontgifting, gaat het hierbij slechts om kleine hoeveelheden glutamine die afgegeven worden naar de bloedbaan. Om die reden moet worden aangenomen, dat het hier niet om een kwantitatief belangrijk mechanisme van ammoniak-ontgifting gaat. Bovendien heeft deze ammoniakontgiftingsroute mogelijk belangrijke consequenties voor de excitatoire neurotransmissie.

De darmen tonen gedurende acuut leverfalen (Hoofdstuk 5) een toegenomen afgifte van ammoniak naar de bloedbaan, terwijl de glutamine-opname afneemt en tot slot verandert in netto glutamine-afgifte. Deze laatste waarneming zou verklaard kunnen worden door inhibitie van glutaminase met daaruit voortvloeiende afname van glutamine-consumptie door de darm, resulterend in dysfunctie van de mucosa-cel. Gedurende chronisch leverfalen (Hoofdstuk 9) is de intestinale ammoniak-afgifte niet toegenomen, suggererend dat in deze situatie de darm niet bijdraagt aan een verergering van de bestaande hyperammoniëmie door een toegenomen glutamine-opname en ammoniak-afgifte.

De resultaten van hoofdstukken 7 en 11 tonen zeer duidelijk aan, dat de rol van de nier in de aanpassing aan zowel chronische als acute hyperammoniëmie ten onrechte onderschat wordt. Zowel gedurende acute als gedurende chronische hyperammoniëmie vermindert de nier de totale hoeveelheid ammoniak geproduceerd en verhoogt de fractie van de totale ammoniakproductie, die in de urine wordt uitgescheiden. Hierdoor verandert de nier van een orgaan, dat netto ammoniak aan het lichaam toevoegt in de normale rat, in een orgaan, dat netto ammoniak uit het lichaam uitscheidt in de hyperammoniemische rat. De resultaten van hoofdstuk 7 suggereren, dat gedurende acute lever-ischemie de capaciteit van de nier om ammoniak te excreteren overschreden is.

De waarneming gedurende acute lever-ischemie, dat de glutamine-afgifte door de skeletspier verminderd is, terwijl de arteriële concentraties van glutamine zijn toegenomen, doet de vraag rijzen van de bron van deze toegenomen arteriële glutamine-concentraties. De slechts minimale hoeveelheden glutamine afgegeven door de hersenen in hetzelfde model kunnen deze stijging van het arteriële glutamine ook niet verklaren. De afgenomen intestinale glutamine opname tijdens acute lever-ischemie is ook geen voldoende verklaring, aangezien het arteriële glutamine reeds gestegen is voor de intestinale consumptie daalt. Aangezien de renale glutamine-consumptie ook niet veranderde gedurende acuut leverfalen, zou misschien een vermeerderde glutamine-afgifte door de longen of het hart, of een verminderde consumptie van glutamine in het immuun-systeem een rol kunnen spelen in deze toegenomen arteriële glutamine-concentraties.

Tenslotte lijkt op grond van de gegevens in dit proefschrift de conclusie gerechtvaardigd dat glutaminase-remming een betere verklaring vormt voor vele van de waargenomen veranderingen tijdens acuut en chronisch leverfalen, dan stimulatie van glutamine-synthetase. 



\section{Dankwoord}

In december 1980 stopte ik met de opleiding Geneeskunde. Ik wilde antiekrestaurateur worden. Mijn vader (Oh Salomons-oordeel) kon daarmee akkoord gaan, maar vond dat ik tijdens mijn zoektocht naar en in afwachting van de start met die opleiding toch gewoon voorlopig door moest gaan met de opleiding Geneeskunde in Nijmegen. Aldus geschiedde. Ik vond geen geschikte opleiding tot antiekrestaurateur, maar wel een baantje bij een antiquair. Toch bleef de ambivalentie in mij bestaan en als een soort bipolaire stoornis kwam bij tijd en wijlen de drang in mij op de Geneeskunde eraan te geven. In 1983 overleed mijn moeder. Voor haar overlijden vroeg ze mij te beloven in ieder geval het doctoraal Geneeskunde te behalen. De studie Geneeskunde werd weer voortgezet en met het klinischer worden van de universitaire opleiding, vond ik ook steeds meer bevrediging in de studie. De belofte aan mijn moeder is inmiddels ingelost. Toch is het niet alleen aan Salomonsoordelen en beloften te danken, dat ik nu ben, waar ik ben.

Peter Soeters, je overredingskracht en enthousiasmerend vermogen waren in staat mij voor zo'n hongerloon het BMC in te krijgen. Ik heb er geen moment spijt van gehad. In het begin dacht ik bij gezamenlijke besprekingen, dat je nooit antwoord gaf op vragen, maar na een tijdje kreeg ik door, dat je gewoon wat langzaam was.

Mick Deutz, onze relatie heeft toch wel wat aspecten van een haat-liefde verhouding gehad. Daarvoor zijn we verschillend genoeg. Je enorme drive en geweldig kritische blik op research en alles, wat daarmee verband heeft, zal ik niet snel vergeten.

Hans, Marion, Mieke J., Gabrie en Annemie maakten mij wegwijs in laboratoriumland en hebben menig bepaling voor mij verricht. Zonder hen had het nooit tot dit proefschrift kunnen komen.

Margitta Kampman en George Athanasas, jullie hielpen bij de totstandkoming van twee hoofdstukken van dit proefschrift, waarvoor mijn hartelijke dank.

Wim Buurman, je gaf vaak ongevraagd menig waardevol advies. Je kritische opmerkingen tijdens werkbesprekingen waren van grote waarde bij de interpretatie van verscheidene experimenten, vooral met betrekking tot het niermetabolisme.

Marc Bemelmans, als maatjes zijn we gestart en zo zullen we nog een poosje verder gaan tijdens de opleiding Chirurgie. Op dieptepunten hebben we elkaar menigmaal opbeurend toegesproken. Eigenlijk was alleen de radio een probleem.

Jeroen Meijerink, samen starten we 'het Isoleucine-onderzoek' in de kliniek. Menig nacht hebben we zo in Ziekenhuis St. Annadal doorgebracht. Ook buiten het ziekenhuis hebben we elkaar niet alleen overdag getroffen. Bedankt voor je kameraadschap.

De 'jongens van het CPV': Harry, Huub, Peter, May, Frans en Eep wil ik heel hartelijk bedanken voor hun hulp bij het verzorgen van de proefdieren. Ton van de Boogaard en de secretaresses van het CPV wil ik graag bedanken voor 'facilitatie'.

De technische dienst van het BMC bedank ik voor het maken van de 'buttons'.

De secretaresses van de Heelkunde, speciaal Marcia, Wilma en Marian wil ik van harte bedanken voor hand- en spandiensten. 
De jongens van het $7^{c}$ voetbalelftal van MKC wil ik bedanken voor de geweldige tijd die we samen hebben gehad op en naast het veld. Op zondagochtend kon ik alle opgekropte agressie van een week promotieleed botvieren op het voetbalveld. Jammer dat een recent ongeval mij verder alleen voor de functie van toeschouwer geschikt maakt.

Trudy, Joop, Maarten, Ingeborg, Mieke D., Eckhardt, Jet en Gaby, jullie waren vaak net klaar met eten, als ik eraan kwam. Ik heb me altijd afgevraagd, of dat nou toeval was.

Sylvia en Steven, menig proefdier hebben we samen geopereerd. Het was een plezier met jullie te werken.

$\mathrm{Nel}$, je was vooral een rustige kamergenote. Alleen als er weer een deadline was, merkte ik echt, dat je er was. Ik realiseer me, dat ik mijn plekje eigenlijk aan jouw gezinsuitbreiding te danken had. Waarvoor mijn dank.

Prof.dr. F.M.J. Debruijne, U stak in 1985 het research-kaarsje in mij aan door mij de kans te geven op de afdeling Urologie van het St. Radboudziekenhuis te Nijmegen enige tijd rond te kijken. Bedankt voor de geboden kans.

Dr. H.L. de Smet, U maakte mij met Uw uitstraling, opvattingen en krasse uitspraken enthousiast voor de chirurgie. Met uitspraken als 'lk opereer pas met zo'n ding als ik er met twee handen door naar binnen kan (over de laparoscoop; Eindhoven, januari 1992)' en 'Van een kras op een buik is nog nooit iemand dood gegaan' won $\mathrm{U}$ in mij weer een zieltje.

De vakgroep Algemene Heelkunde in Maastricht, en speciaal Prof.dr. G. Kootstra, wil ik bedinken voor de geboden mogelijkheden in een prima werkklimaat.

Mijn vrienden, broertjes en andere naaste familieleden heb ik de laatste jaren te vaak in de kou moeten laten staan. Toch was er altijd begrip voor het feit dat ik met het proefschrift be zig was. Ik wil jullie hiervoor hartelijk bedanken.

Mijn paranymfen, Karel en Fons: bedankt voor de hulp en het medeleven.

Lieve Gerard en Corine, jullie waren er altijd als het nodig was met vaak heel nuttige adviezen. Binnenkort komt 'der Bube' weer eens bij 'Nonkelchen' voetbal kijken.

Lieve Ton, ook al stond het onderwerp van mijn promotie ver van je af, je leefde altijd met me mee. In de laatste maanden hielp je veelvuldig door op de kinderen te passen. Hartelijk dank voor alle goeds, dat je ons hebt gegeven.

Jean, lieve $\mathrm{Pa}$, het blijft jammer, dat Anne dit niet mee mocht maken. Jullie hebben ervoor gezorgd, dat dit allemaal mogelijk werd. Niet alleen door beloften en Salomonsoordelen. Dat zou een verkeerd beeld van jullie schetsen. Wat mij vooral bij zal blijven is de warmte, die jullie mij mee hebben gegeven. Dat gaf een gevoel van zelfvertrouwen en het idee, uiteindelijk toch zelf de goede beslissing te hebben genomen.

Lieve Bep, aan de zijde van mijn vader heb je er altijd voor gezorgd, dat we het samen enorm gezellig hadden. Je meeleven met ons wel en wee waardeer ik enorm.

Tot slot, mijn lieve Odile. Jij was er al, toen alles nog moest beginnen. Ik zat nog op de middelbare school, lang haar, strakke T-shirts. De twijfel: houtbewerking of geneeskunde, de dood van mijn moeder, je was er steeds. Je hielp me uit de diepste dalen, ook na die donkere dag in juli 1983. De liederen van Ferrat, Aznavour, de Col de la Croix de Fer, de Mont Sainte Odile, nooit week je van mijn zij, ook niet in de allerlaatste zware maanden. Je schonk me twee dochtertjes en droeg het grootste deel van de zorgen alleen. Ik heb een intens respect voor je, want ik weet, dat ik in jouw plaats al lang naar een ander was gevlucht. Ik leerde je kennen door een gat in de muur. Nu spring ik een gat in de lucht, het zit erop. Morgen beginnen we in een 'Andere Wereld' in een nieuw huis. 


\section{Curriculum Vitae}

Kees Dejong was born on Januari $14^{\text {th }}, 1962$ in Maastricht, The Netherlands. He attended high school in Maastricht, Henric van Veldeke College (Gymnasium B). In 1980 he went to Medical School at the Catholic University of Nijmegen, and graduated in 1987. In may 1988 he started working as a surgical resident at the Surgical Department of the Academic Hospital, University of Limburg, Maastricht (Head: Prof.dr. G. Kootstra). From March 1989 until May 1992 he was a research fellow at the Laboratory for Experimental Surgery, Department of Surgery, Biomedical Center, University of Limburg. In June 1992 he started his surgical training in the Academic Hospital Maastricht. For part of the work presented in this thesis he received an ESPEN Travel Award (Antwerpen, Belgium, 1991). 


\section{PUBLICATIONS (PAPERS)}

N.E.P. Deutz, C.H.C. Dejong, P.B. Soeters. Ammonia and glutamine metabolism during liver insufficiency: the muscle-gut-liver axis. Italian Joumal of Gastroenterology, 1993; in press (Chapter I).

C.H.C. Dejong, N.E.P. Deutz, P.B. Soeters. Inter-organ nitrogen exchange during prolonged starvation in the rat. Joumal of Clinical Nutrition and Gastroenterology, 1991;6:176-183 (Chapter 3)

N.E.P. Deutz, C.H.C. Dejong, G. Athanasas, P.B. Soeters. Partial enterectomy in the rat does not diminish muscle glutamine production. Metabolism 1992; in press (Chapter 4).

C.H.C. Dejong, M.T. Kampman, N.E.P. Deutz, P.B. Soeters. Altered glutamine metabolism in gut and muscle during acute liver failure in the rat. Gastroenterology, 1992;102:936-948 (Chapter 5).

N.E.P. Deutz, C.H.C. Dejong, P.L.M. Reijven, P.B. Soeters. In vivo ammonia and glutamine flux measurements during hyperammonemia in rats and pigs. In: F. Bengtsson, B. Jeppsson, T. Almdal, H. Vilstrup (eds.): Progress in hepatic encephalopathy and metabolic nitrogen exchange. CRC Press, Boca Raton, Florida, U.S.A., pp. 329-339, 1991 (Chapter 5 \& 6).

C.H.C. Dejong, N.E.P. Deutz, P.B. Soeters. A simple method for repeated in vivo cerebral cortex flux measurement in rats. Laboratory Animal Science, 1992;42:280-285 (Chapter 6A).

C.H.C. Dejong, M.T. Kampman, N.E.P. Deutz, P.B. Soeters. Cerebral cortex ammonia and glutamine metabolism during liver insufficiency-induced hyperammonemia in the rat. Journal of Neurochemistry, 1992:59:1071-1079 (Chapter 6B).

C.H.C. Dejong, N.E.P. Deutz, P.B. Soeters. Renal ammonia and glutamine handling during acute liver failure in the rat. Submitted (Chapter 7).

C.H.C. Dejong, N.E.P. Deutz, P.B. Soeters. Muscle amino acid metabolism in two rat models of chronic liver insufficiency: influence of pair-feeding. Submitted (Chapter 8).

C.H.C. Dejong, N.E.P. Deutz, P.B. Soeters. Intestinal glutamine and ammonia metabolism during chronic liver insufficiency-induced hyperammonemia. Gut, 1992; accepted for publication (Chapter 9).

C.H.C. Dejong, N.E.P. Deutz, P.B. Soeters. Cerebral cortex ammonia and glutamine metabolism in two rat models of chronic liver insufficiency-induced hyperammonemia: influence of pair-feeding. Journal of Neurochemistry, 1993; in press (Chapter 10).

C.H.C. Dejong, N.E.P. Deutz, P.B. Soeters. Metabolic adaptation of the kidney to hyperammonemia during chronic liver insufficiency in the rat. Submitted (Chapter II).

N.E.P. Deulz. S. Heeneman, C.H.C. Dejong, W.J.H.J. Meyerink, H.M.H. van Eijk, R. van der Hulst, M.F. von Meyenfeldt, P.B. Soeters. Selective uptake of glutamine in the gastrointestinal tract: confirmation in a human study. British Journal of Surgery 1992; 79:280 (Letter to the editor).

H.M.H. van Eijk. C.H.C. Dejong, N.E.P. Deutz. Influence of storage conditions on the concentrations of free amino acids in rat plasma. Submitted.

S. Heeneman, C.H.C. Dejong, N.E.P. Deutz. Effects of methionine sulfoximine administration on renal ammonia and glutamine metabolism in vivo in the rat. Submitted. 
